\title{
Magnetohydrodynamic Oscillations in the Solar Corona and Earth's Magnetosphere: Towards Consolidated Understanding
}

\author{
V.M. Nakariakov ${ }^{1,2,3}$ • V. Pilipenko ${ }^{4}$ B. Heilig ${ }^{5}$ P. Jelínek ${ }^{6}$ M. Karlický ${ }^{7}$.

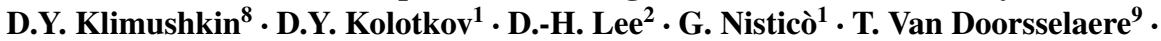 \\ G. Verth ${ }^{10}$ I.V. Zimovets ${ }^{4,11,12}$
}

Received: 4 May 2015 / Accepted: 29 December 2015 / Published online: 2 February 2016

(C) Springer Science+Business Media Dordrecht 2016

\begin{abstract}
Magnetohydrodynamic (MHD) oscillatory processes in different plasma systems, such as the corona of the Sun and the Earth's magnetosphere, show interesting similarities and differences, which so far received little attention and remain under-exploited. The successful commissioning within the past ten years of THEMIS, Hinode, STEREO and SDO spacecraft, in combination with matured analysis of data from earlier spacecraft (Wind, SOHO, ACE, Cluster, TRACE and RHESSI) makes it very timely to survey the breadth of observations giving evidence for MHD oscillatory processes in solar and space plasmas, and state-of-the-art theoretical modelling. The paper reviews several important topics, such as Alfvénic resonances and mode conversion; MHD waveguides, such as the magnetotail,
\end{abstract}

V.M. Nakariakov

V.Nakariakov@warwick.ac.uk

1 Centre for Fusion, Space and Astrophysics, University of Warwick, Coventry CV4 7AL, UK

2 School of Space Research, Kyung Hee University, Yongin, 446-701 Gyeonggi, Korea

3 Central Astronomical Observatory at Pulkovo, St. Petersburg 196140, Russia

4 Space Research Institute, Moscow 117997, Russia

5 Tihany Geophysical Observatory, Geological and Geophysical Institute of Hungary, Tihany, Hungary

6 Faculty of Science, Institute of Physics and Biophysics, University of South Bohemia, 37005 České Budějovice, Czech Republic

7 Astronomical Institute of the Academy of Sciences of the Czech Republic, 25165 Ondřejov, Czech Republic

8 Institute of Solar-Terrestrial Physics, Lermontov St. 126A, Irkutsk 664033, Russia

9 Department of Mathematics, Centre for Mathematical Plasma Astrophysics, KU Leuven, 3001 Leuven, Belgium

10 Solar Physics and Space Plasma Research Centre (SP2RC), University of Sheffield, Sheffield S3 7RH, UK

11 National Space Science Center, Chinese Academy of Sciences, Beijing 100190, China

12 International Space Science Institute, Beijing 100190, China 
coronal loops, coronal streamers; mechanisms for periodicities produced in energy releases during substorms and solar flares, possibility of Alfvénic resonators along open field lines; possible drivers of MHD waves; diagnostics of plasmas with MHD waves; interaction of MHD waves with partly-ionised boundaries (ionosphere and chromosphere). The review is mainly oriented to specialists in magnetospheric physics and solar physics, but not familiar with specifics of the adjacent research fields.

Keywords Magnetohydrodynamic waves (e.g., Alfvén waves) · MHD waves, plasma waves, and instabilities $\cdot$ Magnetic pulsations $\cdot$ Corona $\cdot$ Solar activity $\cdot$ Flares

\section{Introduction}

The solar corona and the Earth's magnetosphere are undoubtedly the most studied natural plasma systems. Both consist of highly ionised plasmas penetrated by a magnetic field, that plays the decisive role in the structure, short-term dynamics and long-term evolution of these plasmas. The solar corona and the Earth's magnetosphere are the key elements of the solar-terrestrial connections and hence attract growing interest in the context of space weather and climate. Moreover, both the corona and the magnetosphere are natural plasma laboratories, where one can find the combinations of physical conditions of broad interest. In particular, there are conditions directly relevant to the efforts in controlled fusion. Also, the corona and magnetosphere are priceless for the investigation of fundamental physical processes operating in natural and laboratory plasmas (e.g. magnetic reconnection, waveparticle interaction, macroscopic and microscopic instabilities, charged particle acceleration, turbulence). One of these basic physical processes is magnetohydrodynamic (MHD) waves. In both the Earth's magnetosphere and the solar corona MHD waves are well observed with satisfactory time and spatial resolution, mainly because of the recently commissioned new generation of space-borne and ground-based observational tools.

MHD waves are propagating or standing perturbations of macroscopic parameters of the plasma (see Alfvén 1942, who was eventually awarded the Nobel prize in 1970). More specifically, MHD waves can perturb the magnitude and direction of the magnetic field, the plasma mass density and associated concentrations of individual species (e.g. electrons and positive ions), plasma temperature and gas pressure. In addition, MHD waves include perturbations of the electric field, electric current, and macroscopic (or bulk) flows of the plasma. Essentially, the presence of MHD waves is connected with MHD restoring forces associated with the magnetic tension and total (gas plus magnetic) pressure, and with the plasma inertia, caused by the frozen-in condition (perpendicular motions of the plasma lead to the change of the magnetic field geometry, and the other way around). MHD waves have been intensively studied in the Earth's magnetosphere for several decades. In the solar corona, the main interest in MHD waves appeared more recently, in the late 90s, with the first observations of these waves with high-resolution extreme-ultraviolet (EUV) imagers on the space missions SOlar and Heliospheric Observatory (SOHO) and Transition Region and Coronal Explorer (TRACE). Both coronal and magnetospheric observations provide us with abundant information about MHD waves. In both the fields, there is a number of elaborated theoretical models addressing specific observational properties of MHD waves. Responding to the intensive research activity in the MHD wave studies, there are a number of comprehensive reviews of different aspects of the topic, see, e.g. Zaitsev and Stepanov (2008), Petrosyan et al. (2010), Banerjee et al. (2011), Patsourakos and Vourlidas (2012), De Moortel and Nakariakov (2012), Stepanov et al. (2012), Mathioudakis et al. (2013), Liu and Ofman (2014) for most recent "solar" reviews, and Guglielmi and Pokhotelov (1996), Walker 
(2005), Alperovich and Fedorov (2007) for "magnetospheric" reviews. Regrettably, in the majority of cases MHD wave phenomena in the solar corona and the Earth's magnetosphere are studied separately. Moreover, cross-talk between these two communities who are studying rather similar plasma environments is complicated by the use of different terminology and different observational techniques.

A comparative study of physical phenomena associated with MHD waves in the corona and magnetosphere provides us with promising basis for deepening our understanding of MHD waves in general. Moreover, the complementarity of the knowledge gained by both these research communities, and exploitation of differences and similarities of MHD wave dynamics in the corona and magnetosphere can bring breakthrough results to both research branches, and is of unequivocal importance for MHD wave studies in other astrophysical, geophysical, space and laboratory plasma systems. The aim of this review is to initiate the mutually beneficial dialogue between the research communities specialising in MHD wave studies in the solar corona and the Earth's magnetosphere. To begin with we shall briefly introduce the basic properties of coronal and magnetospheric plasmas, aiming to establish the common terminology and create the ground for the further discussion. This will be followed by the discussion of specific phenomenology and theoretical modelling. In the end, we describe the similarities and differences between the observed MHD waves. Most notably, in the solar corona the waves are measured through remote-sensing and only the collective oscillation parameters can be measured. On the other hand, in magnetospheric waves insitu parameters are measured, but often the global picture is lacking and no detailed spatial information is available.

MHD wave processes in the terrestrial magnetosphere are observed as ultra-low frequency (ULF) waves in the frequency band from fractions of $\mathrm{mHz}$ to a few $\mathrm{Hz}$. Interestingly, despite very different spatial scales, MHD wave processes observed in the solar corona have frequencies in the same band. We describe both theoretical models and analysis of observational ground and satellite data. We have tried not just to review separately ULF waves in the magnetosphere and solar coronal waves, but to provide, on one hand, observational and theoretical ideas from magnetospheric physics which could be applied in solar physics and the other way around. In addition, we outline unresolved problems, where the experience and expertise gained in the general MHD wave community is of great importance. This review is mainly oriented to specialists in magnetospheric physics and solar physics, but not familiar with specifics of the adjacent research fields. In other words, the solar part is written for magnetospheric physicists, whereas the magnetospheric part-for solar specialists. The comprehensive reference list would be too lengthy and might exceed the review length. Therefore, we have provided references to key papers and reviews only, whereas many important observational studies or historic aspects of a problem have been omitted.

\subsection{What Is the Solar Corona?}

The corona of the Sun is the upper-most part of the solar atmosphere. It consists of almost fully-ionised hydrogen plasma, with about $20 \%$ (by mass) of alpha particles and much lower fraction (less than $2 \%$ by mass) of ions of heavier chemical elements. The corona lies above the partly-ionised and relatively cool region of the solar atmosphere, the chromosphere, where the temperature is a few tens of thousand $\mathrm{K}$, and the mass density drops down with height from about $10^{-8} \mathrm{~g} \mathrm{~cm}^{-3}$ to $10^{-14} \mathrm{~g} \mathrm{~cm}^{-3}$ in about $3000 \mathrm{~km}$. In the corona, the mass density keeps decreasing with height, with the typical values of $10^{-16} \mathrm{~g} \mathrm{~cm}^{-3}$. The corresponding value of the electron concentration is of the order of $10^{8} \mathrm{~cm}^{-3}$. Typically, 
the characteristic scale height is determined by the plasma temperature $T$, and in the hydrostatic equilibrium can be estimated as $H[\mathrm{Mm}] \approx 52 T[\mathrm{MK}]$, where the solar physics units $1 \mathrm{Mm}=10^{6} \mathrm{~m}$, and $1 \mathrm{MK}=10^{6} \mathrm{~K}$.

The temperature of the coronal plasma is higher than several hundred thousand $\mathrm{K}$, reaching several tens of million $\mathrm{K}$ in solar flares. Between the chromosphere and the corona there is a thin transition region (of the width about $0.1 \mathrm{Mm}$ ) where the plasma temperature rises very rapidly with height from several tens of $10^{3} \mathrm{~K}$ to about one million $\mathrm{K}$. The high temperature of the coronal plasma constitutes the "coronal heating problem": it is commonly accepted that it is connected with the conversion of the magnetic energy, while the specific mechanisms are still intensively debated (see, e.g. Reale 2014, for a recent review). The corona extends outwards of the Sun up to several tens of the solar radii $R_{\odot}\left(R_{\odot} \approx 696 \mathrm{Mm}\right)$. But, coronal wave studies are usually limited to the heights up to about one $R_{\odot}$ by the observational constrains. At about several $R_{\odot}$ from the solar surface the corona is usually referred to as the solar wind, the continuous flow of the plasma outwards from the Sun, that reaches outward speeds of several hundreds of $\mathrm{km} \mathrm{s}^{-1}$. The boundary of the corona and the solar wind is not well defined. Perhaps a useful definition of this boundary is the height at which the solar wind becomes supersonic or super-Alfvénic. Likewise, the outer boundary of the solar wind may be considered to be the heliopause, where the solar wind ram pressure equals the ram pressure of the interstellar plasma. In this context, the Earth can be considered to be embedded in the extension of the solar corona-the solar wind plasma flow.

The corona is penetrated by the magnetic field. The field comes from the localised discrete magnetic sources of either positive or negative polarity, situated at the surface of the Sun. Geometrical sizes of the magnetic sources of the same polarity are very much smaller than $R_{\odot}$. For example, the typical size of a sunspot is smaller than $20 \mathrm{Mm}$. There are bigger unipolar sources, called plages, but their size does not exceed 100-150 Mm. In general, both Northern and Southern hemispheres of the Sun have a number of small unipolar sources of the opposite magnetic polarity. Some imbalance between the opposite magnetic polarities form the global polarity: for about 11 years one of the hemispheres is more magnetically "positive" and the other is more "negative". Then the polarity swaps. The chain of these swaps forms the solar cycle. The time intervals of the swaps are nearly concurrent with solar cycle maxima, separated by the minima of the opposite magnetic polarity, which are defined as the period when the average number of sunspots, hence the magnetic source, is minimal. For a comprehensive review of long-term solar variability, see e.g. Hathaway (2010).

In the corona, the magnetic field created by different sources at the solar surface is believed to fill up all the volume. Thus, the magnetic configuration of the solar corona, especially at the altitudes lower than $0.5 R_{\odot}$, is very complex and essentially not dipolar. One can distinguish between closed coronal magnetic configurations that are created by the magnetic field lines beginning and ending at the surface of the Sun, and open magnetic configurations in which the field lines go to or come from (depending upon the direction of the field) the outer space.

Some magnetic flux tubes are filled in with dense plasma. In the closed magnetic configuration such plasma structures have loop-like geometry, highlighting arching magnetic field lines and emitting strongly at EUV and soft X-ray wavelengths (see Fig. 1). Typically coronal (torus-shaped) loops have major radii shorter than $100 \mathrm{Mm}$, and their minor radii are believed to be about $1 \mathrm{Mm}$. However the latter estimate is still debated, and it is possible that loops are bundles of unresolved thin threads. Loops are usually clustered in certain locations (e.g. above sunspots), forming bright active regions. Simultaneously, there can be several active regions in the corona, more in time intervals of the solar cycle maxima. In open 




Fig. 1 A typical active region of the solar corona, situated at the solar limb and observed at six EUV wavebands of SDO/AIA. Colours in each panel are artificial, with the brightest pixels corresponding to the biggest number of EUV photons of the prescribed wavelength and hence to the denser plasma of the selected temperature

magnetic configurations, the magnetic flux tubes filled in with the dense plasma are known as plumes, and they are usually observed in coronal holes, the fainter parts of the corona. Thus, the coronal plasma is filamented along the local, nearly radial magnetic field in the density and temperature in both regions of closed and open magnetic geometry. The density contrast inside and outside the loop is usually 3-5, but it can reach 100 in flaring sites. EUV loops are usually seen to be of constant cross-section, and without noticeable twist. Hence, the magnetic field along the loop is usually considered to be constant at all heights. This effect is subject to intensive debate, as the field should generally decrease with the distance from the Sun. In particular, Malanushenko and Schrijver (2013) suggested that the apparent constant width could be caused by the effect of non-circular cross-sectional shape of the loops. Another possible solution to this problem is to assume that the observed EUV loops are not cospatial with magnetic flux tubes (Peter and Bingert 2012), while the relevant theory needs to be developed. There is observational evidence that even in the regions that are seen as diffuse, the plasma is still filamented along the field (e.g. Van Doorsselaere et al. 2008 b). The typical values of the magnetic field in the lower corona (lower than $R_{\odot}$ above the surface of the Sun) is from a few $\mathrm{G}$ in open magnetic structures, e.g., coronal holes, to a few hundred $\mathrm{G}$ in sunspot magnetospheres. The value of the plasma parameter $\beta$ in active regions and in coronal holes is very small, about a few percent. In the diffuse closed corona and in flaring loops it can perhaps reach 0.5 (e.g. Schmidt and Ofman 2010; Shibasaki 2001, respectively).

An important parameter of the corona is the ion collision frequency. Using the estimating expression (Book 1983)

$$
v_{\mathrm{i}}\left[\mathrm{s}^{-1}\right] \approx \frac{96 \times 10^{-8} n_{\mathrm{e}}\left[\mathrm{cm}^{-3}\right]}{T[\mathrm{eV}]^{3 / 2}},
$$


where the Coulomb logarithm is taken as 20 , and the plasma is neutral and fully ionised. For a typical coronal loop with $n \approx 10^{9} \mathrm{~cm}^{-3}$ and $T \approx 10^{6} \mathrm{~K} \simeq 80 \mathrm{eV}$ we get that the typical time between collisions is $\approx 1 \mathrm{~s}$. Hence, for the oscillatory processes with the periods $P>5-10 \mathrm{~s}$, it should clearly be sufficiently collisional in the direction along the field. In the direction across the field the role of collisions is played by gyration. The proton cyclic gyrofrequency can be estimated as $\Omega_{\mathrm{i}}\left[\mathrm{rad} \mathrm{s}^{-1}\right] \approx 10^{4} B[\mathrm{G}]$. For a typical coronal loop with $B=20 \mathrm{G}$ the proton gyrofrequency is $f_{\mathrm{i}} \approx 30 \mathrm{kHz}$. Thus, coronal plasma is highly magnetised. In an active region loop with $B=20 \mathrm{G}$ and $T=10^{6} \mathrm{~K}$ the proton gyroradius is about $r_{\mathrm{i}} \approx 50 \mathrm{~cm}$, while the loop typical minor radius is $1 \mathrm{Mm}$. The Debye shielding distance is typically $0.1 \mathrm{~m}$ in a coronal active region.

The corona is a highly-variable medium at time scales from a second to several years. Typical time scales of MHD waves and oscillations are between a fraction of a second and several hours. A similar time scale is the lifetime of various jets. For comparison, the typical time scale of thermodynamic evolution of coronal loop brightness is several hours. The typical lifetime of a coronal loop is from a few hours to a few days. The period of solar rotation is about 4 weeks. Active regions are long-living objects, existing in the corona for up to several weeks. Longer period evolution of the corona, with typical periods from several months to several years or longer, is associated with the solar cycle and is not discussed in this review.

The corona is the place of origin of impulsive energy releases, including flares and coronal mass ejections (CMEs) besides various jets. Comprehensive reviews of recent achievements in the observational study and theoretical modelling of these phenomena can be found by Benz (2008), Chen (2011), Shibata and Magara (2011), Webb and Howard (2012). In this review it is important to mention that flares and CMEs can induce MHD waves and oscillations. Indeed, any localised impulsive energy release, such as a flare or CME, in an elastic and compressive medium, such as the magnetised plasma of the solar corona, would excite waves. In turn, MHD waves, as macroscopic perturbations of plasma parameters, can trigger the energy releases by destroying the equilibrium in sub-critical plasma configurations. Moreover, light curves of solar flares, that are time-dependencies of the solar emission recorded in different observational bands, show quasi-periodic variations. These oscillations with the periods from a fraction of a second to several minutes are usually referred to as quasi-periodic pulsations (QPPs) and are also associated with MHD processes (Nakariakov and Melnikov 2009).

The observational knowledge about the coronal plasma is mainly obtained in the EUV and soft X-ray bands, that correspond to the black-body radiation of a very hot plasma, of the temperature more than one million K. Emission lines of ionised rare elements, such as $\mathrm{Fe}, \mathrm{O}, \mathrm{Mg}$, etc., are well seen in the spectrum. The emission is usually in the optically thin regime, and the brightness of a line is proportional to the square of the plasma density integrated along the line-of-sight $(\mathrm{LoS})$. The emitting plasma should have the temperature about the formation of the considered ion. For example the peak formation temperature of the line $171 \AA$, which is produced by the ion Fe IX, is about $6.3 \times 10^{5} \mathrm{~K}$. If the emission comes from an isolated plasma structure localised along the LoS, the line intensity is proportional to the product of the plasma density squared and the column depth of the structure. EUV imaging telescopes, such as SDO/AIA and TRACE, usually observe the corona in sufficiently narrowband spectral channels that include one selected emission line, and hence give us the spatial distribution of the plasma of a certain temperature corresponding to the formation temperature of this line (see Fig. 1). Broadband imagers, such as Yohkoh/SXT and Hinode/XRT, usually operate in the soft X-ray band and measure the emission of hotter plasmas with temperatures up to 15-30 MK. These instruments give us information about the spatial distribution of the hottest plasma in the corona. 
Spectral instruments, such as SoHO/SUMER and Hinode/EIS, allow us to resolve spectral details of emission lines in a certain band. This makes it possible to measure the Doppler shift and non-thermal broadening of various lines, and hence provide us with the information about the LoS component of the emitting plasma velocity and its unresolved small-scale motions. There are also detectors of high energy photons, such as RHESSI that gives the energy distribution of the X-ray emission from $3 \mathrm{keV}$ to $20 \mathrm{MeV}$ in solar flares, and spatial location of its sources. Radio instruments give time variation of coherent (e.g. plasma) and non-coherent (e.g. gyrosynchrotron and thermal free-free) emission in the bands from tens of $\mathrm{MHz}$ to tens of GHz. Radio interferometers (radioheliographs), such as NoRH, NRH and SSRT, give also the spatial structure of the emission sources.

Time resolution of solar coronal observational facilities reaches milliseconds in the radio band, and several $\mathrm{s}$ in the EUV band, ${ }^{1}$ and sub-second cadence around the optical band. Spatial resolution reaches $0.5^{\prime \prime}$ in the EUV band and $5^{\prime \prime}$ in the radio band (an angular resolution of $1^{\prime \prime}$ corresponds to a spatial scale of about $730 \mathrm{~km}$ on the solar disk, for Earth-bound telescopes). Duration of observations in the best cases is determined by the life time of the oscillating structures. The longest duration used to be limited by the solar rotation, by the time the oscillating structure passing across the solar disk, about two weeks. But, in recent years, the twin spacecraft of the STEREO mission provide us with two additional vantage points, allowing for even longer imaging observations in the EUV band. Also, this space mission makes possible quasi-stereoscopic measurements necessary for the determination of the 3D geometry of coronal plasma configurations.

In solar coronal physics, MHD waves and oscillations have been discussed for several decades, mainly in the context of their possible contribution to coronal plasma heating and solar wind acceleration, and as possible mechanisms for quasi-periodic pulsations in solar flares. In late 90s, commissioning of spaceborne high-resolution EUV imagers allowed for the direct observations of coronal MHD waves, resolving them simultaneously in time and space. These observations provided the coronal research community with a solid observational basis for the confident identification of different kinds of MHD modes, and resulted in the rapid development of the novel technique for the coronal plasma diagnostics by means of coronal MHD waves, i.e. MHD coronal seismology.

\subsection{What Is the Earth's Magnetosphere?}

In the following outline of the magnetosphere structure, we will pay attention mostly to the magnetospheric features which are important for the ULF wave excitation, propagations and transformation processes.

\subsubsection{Outline of the Earth's Magnetosphere}

The magnetosphere is a plasma cavity formed due to the confinement of the Earth's magnetic field by the flow of a highly conductive solar wind plasma (Fig. 2). As a result of the interaction of this super-sonic and super-Alfvénic flow with the Earth's magnetic field a paraboloid-type bow shock is formed in front of the Earth. The average bow shock standoff distance is about $15 R_{\mathrm{E}}$ (sizes in the magnetosphere are often measured by the mean radius of the Earth, $R_{\mathrm{E}}=6371 \mathrm{~km}$ as a unit).

\footnotetext{
${ }^{1}$ For example, the nominal cadence time of the Atmospheric Imaging Assembly on the Solar Dynamics Observatory is 12 seconds, while there is a technical possibility to reduce it to 2 seconds for selected wavelength channels (Lemen et al. 2012). The High Resolution Coronal Imager (Hi-C) on a sounding rocket had the cadence of $5.57 \mathrm{~s}$ (Kobayashi et al. 2014).
} 
Fig. 2 A sketch of the Earth's magnetosphere structure

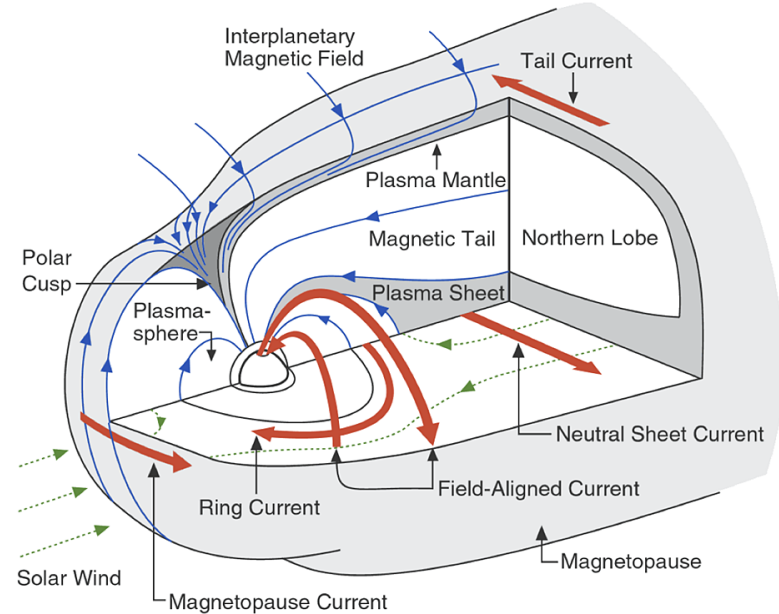

The layer between the bow shock and the magnetosphere, where the super-sonic solar wind slows down to sub-sonic speed, is called the magnetosheath. The sheath is a flow of highly turbulent warm plasma $(\beta \geq 1)$, dragging a weak magnetic field of interplanetary origin $(\sim 10 \mathrm{nT})$ with a density $2-4$ times the density of the solar wind near the Earth's orbit, which is about $20 \mathrm{~cm}^{-3}$.

The outer boundary of the magnetosphere surrounding the region dominated by the magnetic field of terrestrial origin - the magnetopause, is a discontinuity characterised by a steep variation of plasma parameters and magnetic field across it. Its position depends on the dynamic pressure of the solar wind and the direction of the interplanetary magnetic field (IMF). Under average conditions this is about $10-11 R_{\mathrm{E}}$ from the centre of the Earth. The magnetopause is a natural reflective boundary for magnetospheric MHD modes.

On the nightside of the magnetosphere the magnetotail is formed by the solar wind flow, similar to a laminar wake formed behind an obstacle in a fast moving gas/fluid flow. The magnetospheric magnetic field from the polar caps extends into the magnetotail up to few hundreds $R_{\mathrm{E}}$.

Closer to the Earth the magnetic field of the magnetosphere can be well approximated by a dipole field. The distortion of the field line geometry from the dipolar shape increases with the increasing distance from the Earth, especially in the tail. The distortions are caused by magnetospheric current systems driven directly or indirectly by the varying speed of the solar wind and the IMF direction.

Though being a unified system, the magnetosphere may be categorised by two main plasma domains with very different properties:

- the outer magnetosphere with a rarefied plasma $\left(\sim 0.1-1 \mathrm{~cm}^{-3}\right)$ in a weak magnetic field $\left(<10^{2} \mathrm{nT}\right)$, so with a finite- $\beta$ value, $\beta \geq 1$;

- the inner magnetosphere filled with a cold plasma $(\beta \ll 1)$ embedded in a quasi-dipole geomagnetic field.

Dayside reconnection drives a sunward plasma transport (convection) in the outer magnetosphere by generating a dawn-to-dusk electric field (the convection electric field). Similarly, the corotation of plasma with the Earth, as viewed from a frame of reference not corotating with Earth, is caused by the corotating electric field. By summing the potentials associated with the two electric fields, the outermost equipotential surface defines a bound- 
ary separating the corotating plasma from the drifting plasma of the outer magnetosphere, known as the plasmapause. The cold corotating plasma of the inner magnetosphere (up to 4-5 $R_{\mathrm{E}}$ ) is engulfed into a joint rotation with the Earth, which forms a cold plasma torus around the planet, named the plasmasphere, with plasma concentration $n \simeq 10^{2}-10^{4} \mathrm{~cm}^{-3}$. The plasmasphere is filled up with plasma by diffusion from the underlying ionosphere. At the plasmapause a rapid drop of density by around an order of magnitude occurs. The plasmapause is a very dynamic boundary and its position is sensitive to geomagnetic activity. During disturbances, increased convection in the outer magnetosphere erodes the plasmasphere, moving the plasmapause closer to the Earth. Plasma depletion caused by geomagnetic storms is a relatively fast process compared to the refilling that can last several days, even weeks.

The plasma in the magnetosphere consists overwhelmingly of protons and electrons, with some contributions of heavier ions of ionospheric origin $\left(\mathrm{NO}^{+}\right.$and $\left.\mathrm{O}^{+}\right)$, and $\mathrm{He}^{2+}$ of solar wind origin.

The outer part of the magnetotail filled with rarified plasma $\left(n \simeq 10^{-2} \mathrm{~cm}^{-3}, B \simeq 30 \mathrm{nT}\right)$ is the lobe. The lobe surrounds a denser part of the tail, the plasmasheet $\left(n \simeq 0.5 \mathrm{~cm}^{-3}\right.$, $B \simeq 10 \mathrm{nT}$ ), which is about $10 R_{\mathrm{E}}$ thick. The magnetic field lines of the plasmasheet's inner boundary are connected to the Earth's near the poles, i.e. at high latitude auroral regions. In the central part of the plasmasheet, where the reversal of the magnetic field direction occurs, a thin current sheet is formed. This region is very dynamic, and permanently disturbed by magnetic reconnection, bursty bulk flows, various instabilities, etc.

The almost fully ionised near-Earth plasma is separated from the neutral atmosphere by an interface formed of partially ionised plasma known as the ionosphere. Its high density and the partial ionisation has an important consequence: the conductivity of the ionosphere, in contrast to the magnetosphere or the solar wind, is finite. The ionosphere is not a smooth transition layer, but a non-monotonic altitude-dependent structure. Two layers, where the ionisation and recombination rates match, are of special importance for ULF waves: the $F$-layer in the upper ionosphere (at the heights $h \simeq 400 \mathrm{~km}$ from the Earth's surface), where the electron density peaks, and the E-layer in the bottom ionosphere ( $h \simeq 120 \mathrm{~km}$ ). The ionospheric Pedersen and Hall conductivities, and therefore the ionospheric horizontal currents, are concentrated in the E-layer. The ionospheric conductive layer can be the inner reflective/absorbing boundary for magnetospheric MHD waves.

\subsubsection{The Magnetosphere as a Zoo for MHD Wave Species}

Vast plasma volumes bounded by the inner and outer boundaries make the magnetosphere a natural MHD resonator and waveguide, capable to trapping and guiding various global MHD modes. The plasma/magnetic field parameters vary in the radial direction more rapidly than in the azimuthal direction, thus forming an open waveguide around the planet. An evident contrast between the coronal and magnetospheric plasmas is that structuring of the magnetospheric plasma into field-aligned plasma tubes is not so clearly pronounced as in the solar corona.

The interaction of the solar wind flow with the Earth's magnetosphere, which controls the space weather in the near-planetary space, has a non-steady and turbulent character. Thanks to the occurrence of natural MHD waveguides and resonators all dynamic processes in the near-Earth space are accompanied by appearance of electromagnetic waves and oscillations in the ULF frequency band covering three orders in frequency, from fractions of $\mathrm{mHz}$ to a few Hz. ULF pulsations detected by ground or satellite magnetometers are manifestations of MHD waves in near-Earth environment. These waves penetrate through the near-Earth 
space and convey information to the ground about the properties of space environment and its dynamic processes.

In fact, geomagnetic pulsations detected with the help of an oscillating magnetic needle were the first electromagnetic waves detected by mankind. Oscillations of the geomagnetic field were first recorded in 1840s in Bogenhausen by Johann von Lamont, and sometime later, in 1859 by Balfour Stewart. It was recognised only a hundred years later that these pulsations are the ground counterparts of MHD waves in the magnetosphere. According to the energy content, ULF waves are the most powerful electromagnetic wave process in nearEarth space among all space emissions. The ULF frequency interval is bounded from low frequency by the lowest possible eigen oscillations of the entire magnetosphere, whereas from high frequency it is limited by the gyrofrequency of magnetospheric ions in the equatorial plane of the magnetosphere. In ground observations, the ULF band occurs between substorm-related magnetic variations (with time scales about 10 minutes or more) and the Schumann resonance at $f \simeq 8 \mathrm{~Hz}$, the global resonance of the cavity between the lower ionosphere and ground.

For the study of space plasma processes ULF waves are of the same importance as seismic waves for the study of Earth interiors: they enable the remote sounding and monitoring of regions unavailable for in-situ measurements. From a realm of various periodic disturbances in near-Earth space plasma only for a very limited number of emission types we may say with confidence that their physical mechanism is well understood. Nonetheless, the possibility exists to use ULF waves detected on the ground for the hydromagnetic diagnosticsthe determination of the magnetosphere plasma density, "magnetoseismology"- the magnetospheric analogue of the MHD seismology of the solar coronal plasma.

Compared with purely hydrodynamic waves travelling through a non-magnetised medium, MHD waves have a number of distinct features. For example, resonant effects have a principal significance: the resonant wave-particle interaction, and formation of resonant structures in waveguides or resonators. The terrestrial magnetosphere may be considered as a natural plasma laboratory open to the in-situ observations, so the ideas elaborated for this medium could be applied for studies of the Sun or other astrophysical objects.

The theoretical studies of ULF wave phenomena in space plasmas are based on the mathematical approaches and theoretical methods developed in the general plasma physics and magnetohydrodynamics. However, it would be incorrect to think that space plasma physics just applies the physical notions earlier elaborated in a general plasma physics. A number of principally new physical ideas came to the general plasma physics from the space geophysics: e.g. magnetic field line reconnection and magnetic field annihilation, collisionless shock waves, etc. In this list one should also add MHD waves. The MHD mode conversion via the Alfvén resonance that was originally suggested for the interpretation of the latitudinal structure of ULF pulsations in the Earth's magnetosphere, nowadays is widely used in the controlled nuclear experiments for the radio wave plasma heating (e.g. Vaclavik and Appert 1991). The same notions were applied to the interpretation of the mechanism for solar corona heating by MHD waves (see, e.g. Arregui 2015, for a recent review).

\subsubsection{Magnetosphere as a Particle Trap and Accelerator}

The magnetospheric magnetic field is a trap not only for MHD waves, but also for energetic particles. The relatively strong magnetic field $\left(\sim 10^{2}-10^{4} \mathrm{nT}\right)$ of the inner magnetosphere controls the dynamics of energetic electrons and protons injected into the magnetosphere from the magnetotail during substorms. The motion of charged particles in the magneto- 
sphere consists of three kinds of motions: the Larmor rotation around the field line with the gyrofrequency $\Omega_{\mathrm{i}}$, oscillations along a closed field line between the mirror points with the bounce frequency $\omega_{\mathrm{b}}$, and the drift motion in the azimuthal direction with the speed $U_{\mathrm{d}}$ and the angular speed $\omega_{\mathrm{d}}$. Upon the drift around Earth, positively charged particles drift in the westward direction, and negatively charged particles drift in the eastward direction.

Most of the energy of the trapped radiation is in the ring current particles-westward drifting protons with energies 10-200 keV. During magnetic storms the ring current causes a significant depression of the magnetic field inside the current. On the ground, this depression is measured at near-equatorial magnetic stations as Dst-variations, reaching 300-400 nT by the absolute value during the most intense storms. Thus, the plasma composition outside the plasmasphere is a variable mixture of cold "core" and energetic "tail" particles. Near the maximum of the ring current a local distortion of the magnetic field may become significant as $\beta \geq 1$.

The energy provided to the magnetosphere by the solar wind flow with the embedded IMF is accumulated in the magnetotail, and then from time to time spontaneously is released into the inner magnetosphere during substorms. The mechanism for a global instability resulting in the substorm explosive onset is not fully understood yet. There seems to be a striking similarity in the development of "cosmic quakes": magnetospheric substorms and solar flares. A characteristic feature of a substorm is the injection and precipitation of nonthermal energetic electrons into the upper atmosphere. The lower-energy part $(0.1-1 \mathrm{keV})$ of the precipitated electrons produces an intensification of aurora, whereas their higher energy part (10-100 keV) causes additional ionisation of the lower ionosphere. However, particles with energies sufficient to drive the aurora cannot be directly supplied by the low-energy solar wind. Auroral electrons are accelerated by still not well understood mechanisms during the process of releasing energy that has accumulated in the magnetotail, possibly related to magnetic reconnection.

The closed magnetosphere is even more effective natural particle accelerator, and is capable of energising electrons up to relativistic energies $(\geq 1 \mathrm{MeV})$, thus forming the outer radiation belt. The observations show the existence of radiation belts that contain two populations of highly energetic particles, separated by a slot region at about $2-4 R_{\mathrm{E}}$. The inner belt consists mainly of non-thermal energetic protons, while the outer belt is filled in with relativistic electrons. The appearance of relativistic electrons in the magnetosphere, which follows some geomagnetic storms, resists definitive explanation despite many years of study. These electron events are not merely a curiosity for scientists, but they can have disruptive consequences for geosynchronous spacecraft. While it has been known that there is a general association between geomagnetic storms and electron enhancements at the geosynchronous orbit, the wide variability of the observed response and the puzzling time delay (about 1-2 days) between the storm main phase and the peak of the response has frustrated the identification of responsible mechanisms. The enhancements in the electron energies (beyond levels expected from conserving adiabatic invariants) at the geosynchronous orbit occur rapidly at the onset of a magnetic storm, often within a few hours, but there is also a slower additional acceleration, so that peak fluxes are often seen only after a number of days.

Since the solar wind does not directly interact with the electrons in question, some magnetospheric intermediary must more directly provide the electrons with energy. ULF waves in the Pc5 band (the nomenclature of different magnetospheric pulsations is given below) have emerged as a possible energy reservoir for the electron acceleration. It was proposed that the gradual slow energisation of seed electrons supplied by substorms is due to the resonant interaction of drifting electrons with MHD oscillations in the Pc5 frequency range. This 
drift-resonance mechanism operates like a "geosynchrotron". Many observations favoured the idea of a ULF contribution to the later, slower energisation of electrons.

The dynamics of the ring current and outer radiation belt are determined by a delicate balance between the injection/acceleration and loss/precipitation processes. ULF wave activity contributes significantly to these processes in the collisionless magnetospheric plasma. For example, electromagnetic ion-cyclotron (EMIC) waves can control the decay rate of the Earth's outer radiation belt and ring current. Pitch-angle scattering by EMIC waves might account for the rapid depletion of $\mathrm{MeV}$-electron fluxes, limit lifetime of energetic ring current protons, cause selective precipitation of ambient relativistic electrons from the radiation belt, and produce a localised proton aurora.

Today magnetospheric ULF waves are observed either by isolated magnetic observatories or extended ground magnetometer arrays (such as CARISMA, MACCS, McMAC, and SAMBA in the American continent, MAGDAS in the Japanese-Australian sector, IMAGE and EMMA in Europe, just to name a few, for a more complete list visit the SuperMAG website at http://supermag.jhuapl.edu/). The total number of currently operating magnetometers exceeds 200.

Ground observations are complemented by a rapidly growing flow of data from satellite missions. Observations of ULF wave activity is the dedicated goal of some of the missions, however any satellite having a magnetometer with a sufficient sensitivity and resolution onboard can yield valuable data for ULF wave research too. The most valuable data come from multi-satellite missions, like Cluster, THEMIS, VanAllen, making observations in different regions of the magnetosphere or in the solar wind simultaneously. Satellites in geosynchronous orbit (at $6.6 R_{\mathrm{E}}$ ) fixed in the local time are widely used for space weather monitoring and prediction (e.g. GOES and LANL series). For investigation of the interaction of ULF waves with the ionosphere, data from low-Earth-orbiting satellites (e.g. CHAMP, SWARM) are the most valuable. Solar wind monitors at the libration (L1) point, about $200 R_{\mathrm{E}}$ upstream of the Earth (e.g., WIND, ACE), are important not only for providing data on interplanetary conditions, but also because they inform us about waves and turbulence inherent in the solar wind.

Recently, various high time-space resolution radars, such as SABRE, STARE, SuperDARN, EISCAT, have become widely used tools for detecting ULF waves interacting with the ionosphere, where such waves transfer energy through the coupled magnetosphereionosphere system. The periodic signatures in the ionospheric plasma velocity in the ULF band were interpreted as a result of incidence on the ionosphere of MHD waves. Moreover, radar observations have turned out to be an effective tool to detect small-scale ULF waves, which are shielded by the ionosphere from ground magnetometers.

Classification of ULF pulsations is based purely on their morphological features, irrespective of their generation mechanism. Pulsations are divided into two groups: Pc-type pulsations, which are more regular, continuous structured oscillations; and the impulsive, irregular pulsations, termed Pi pulsations (see Troitskaya and Gul'elmi 1967; Dungey and Southwood 1970, for detailed reviews). Both groups are further divided into numbered subgroups according to their period (Pc1: 0.2-5 s, Pc2: 5-10 s, Pc3: 10-45 s, Pc4: 45-150 s, Pc5: 150-600 s, Pc6: longer than 600 s; Pi1: 1-40 s, and Pi2: 40-150 s, Pi3: longer than $150 \mathrm{~s})$. Besides this classification certain ULF phenomena are referred with other historical terms: e.g. the giant pulsations $(\mathrm{Pg})$ are a subclass of Pc4 pulsations characterised by large amplitude (10-50 nT) and localised in the auroral zones, which are believed to be produced by plasma instabilities. Ps 6 pulsations with periods of 600-2400 s are observed during substorms, and are associated with fluctuating electric currents flowing in the ionosphere. 


\subsubsection{Main Differences Between the Corona and Earth's Magnetosphere}

The solar corona and terrestrial magnetosphere are seemingly very different plasmas: plasma of the low corona is collisional (see the estimations in Sect. 1.1, showing that typical collision times much shorter than the observed wave periods), whereas the magnetospheric plasma is collisionless. Nonetheless, MHD approach works well for both those two opposite extreme situations. MHD theory was elaborated to describe motion and waves in a highlyconductive fluid, such as highly-collisional plasma of the solar corona. However, even in a collisionless plasma, such as the near-Earth plasma environment, the single-fluid MHD approach can be applied for large-scale wave processes owing to a nearly-infinite field-aligned conductivity (e.g. Kadomtsev 1982). Only for small-scale processes in the magnetosphere (a relevant scales are given in Sect. 12) the assumption of the infinite field-aligned conductivity, and hence the applicability of the MHD approach, may be violated. These small-scale ULF waves, as well as processes where large-scale wave interaction with a small group of resonant particles are involved, should be described by the kinetic approach, with the use of the Vlasov equation.

Concerning the ion cyclotron frequency, frequencies of both MHD oscillations in the corona and the long-period part of ULF waves in the magnetosphere (e.g., Pc3-5 and Pi2-3 pulsations) are much lower than it. Only high-frequency part of magnetospheric ULF waves (e.g., Pc1 and Pi1 pulsations) has the frequencies approaching the lowest values of the magnetospheric proton cyclotron frequency, near the tops of the magnetic field lines. These pulsations are associated with electromagnetic ion-cyclotron waves.

Another important difference is connected with the minor species, which should be taken into account in the consideration of low-frequency cyclotron resonances (e.g. Cranmer 2002). If in the corona the heavy elements are highly ionised (e.g. Fe IX, He II, O VI), in the magnetosphere the lower ionisation states, e.g. O I and He I are abundant.

Observational approaches in these two plasma environments are very different too. The main difference compared to coronal MHD wave observations is that in the magnetosphere and on the ground, observations are in-situ point observations, while solar observations are obtained from remotely sensed images of the phenomenon as a whole. Thus, coronal observations allow us to study simultaneously the time and spatial evolution of the waves, e.g. resolving both the wavelength and the wave period. However, these observations may be affected by insufficient time and spatial resolution, and are affected by optically-thin effects, i.e. the integration along the line of sight. On the other hand, magnetospheric observations are usually not restricted by the resolution, but may lack the spatial information.

Thus, the similarity between the solar coronal and Earth's magnetospheric plasmas should be exploited with serious caution. There is a number of fundamental physical differences that need to be taken into account. Nevertheless, despite the important differences of these two plasma environments, in the following discussion we attempt to establish the ground for the consolidated understanding of MHD wave processes in the magnetosphere and the corona.

\section{Global Propagating Waves in Solar Corona}

\subsection{Observations of Global Coronal Waves}

There is not any observational evidence of global oscillations of the solar corona, similar to the low-degree acoustic oscillations of the solar surface. The largest example of the coronal MHD wave activity is propagating global coronal waves. The first detection of global coro- 

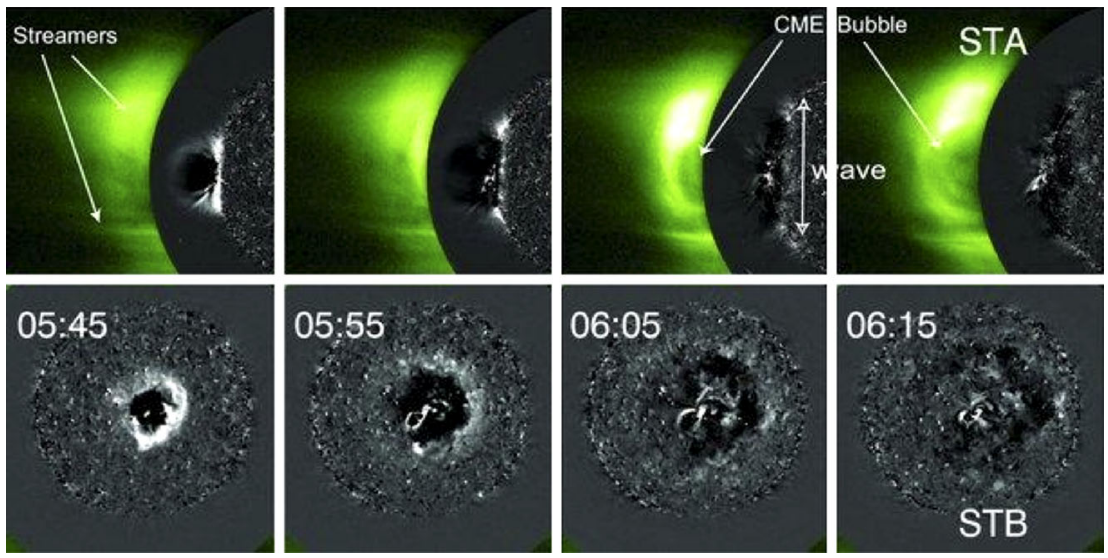

Fig. 3 First quadrature observations from STEREO of a CME-EUV wave event. Top panel: temporal sequence of COR1-EUVI $195 \AA$ images from STEREO-A, showing the evolution of the wave on the solar disk and the CME in the coronagraph from a lateral perspective. Bottom panel: front view of the wave from STEREO B, with a clear circular wave front. From Patsourakos and Vourlidas (2009)

nal waves was achieved by the Extreme ultraviolet Imaging Telescope (EIT) aboard SoHO, which was launched in 1995. Large-scale coronal disturbances propagating in the form of arc or circular bright fronts were captured in the images at $195 \AA$ (see Fig. 3). These disturbances, also referred to as coronal bright fronts $(\mathrm{CBF})$, are usually generated after strong impulsive releases of energy and cover a significant portion of the solar disk, comparable with the solar disk radius. The perception of a propagating wave is additionally enforced by a trailing and expanding dimming region (signature of the evacuation of the plasma), highlighted using a running difference method. They were named as "EIT waves", in virtue of the instrument that has permitted their discovery (Thompson et al. 1999), and were immediately interpreted as the manifestation of a fast-magnetoacoustic mode, i.e. a compressive wave propagating quasi-perpendicularly to the direction of the local magnetic field (e.g. Wang 2000; Ofman and Thompson 2002). Almost twenty years from their discovery, a great debate about their nature, formation and relationships to flares and CMEs is still ongoing. This debate has been further stimulated by observations from the follow-up and currently operative space missions, e.g. STEREO and SDO. For this reason, EIT waves are now referred with the generic name of "EUV waves" or global coronal waves (Fig. 3). For a detailed perspective on the topic, the reader is invited to refer to the reviews of Gallagher and Long (2011), Patsourakos and Vourlidas (2012) and references therein.

The first possible identification of large-extended disturbances propagating in the solar atmosphere should actually be moved backward of thirty years. Indeed, observations in the $\mathrm{H} \alpha$ channel of the chromosphere, which is the layer just below the corona, led to detecting large-scale arc-shaped fronts propagating away from a flaring region at very high speeds ranging between $500-1500 \mathrm{~km} \mathrm{~s}^{-1}$ (for comparison, chromospheric sound and Alfvén speeds are about one or two orders of magnitude lower). These rapidly-propagating chromospheric disturbances are known as Moreton waves, after the name of the discoverer (Moreton 1960), and they were interpreted and modelled in terms of a fast MHD shock that propagates in the corona and sweeps through the underneath chromosphere. The wave, refracted towards a low Alfvén velocity region, sharpen into an enhanced fast-mode shock wave that could emit type II radio bursts (Uchida 1968) (which are slowly-drifting spectral features that are usually associated with electrons accelerated by the shock front). Thus, 

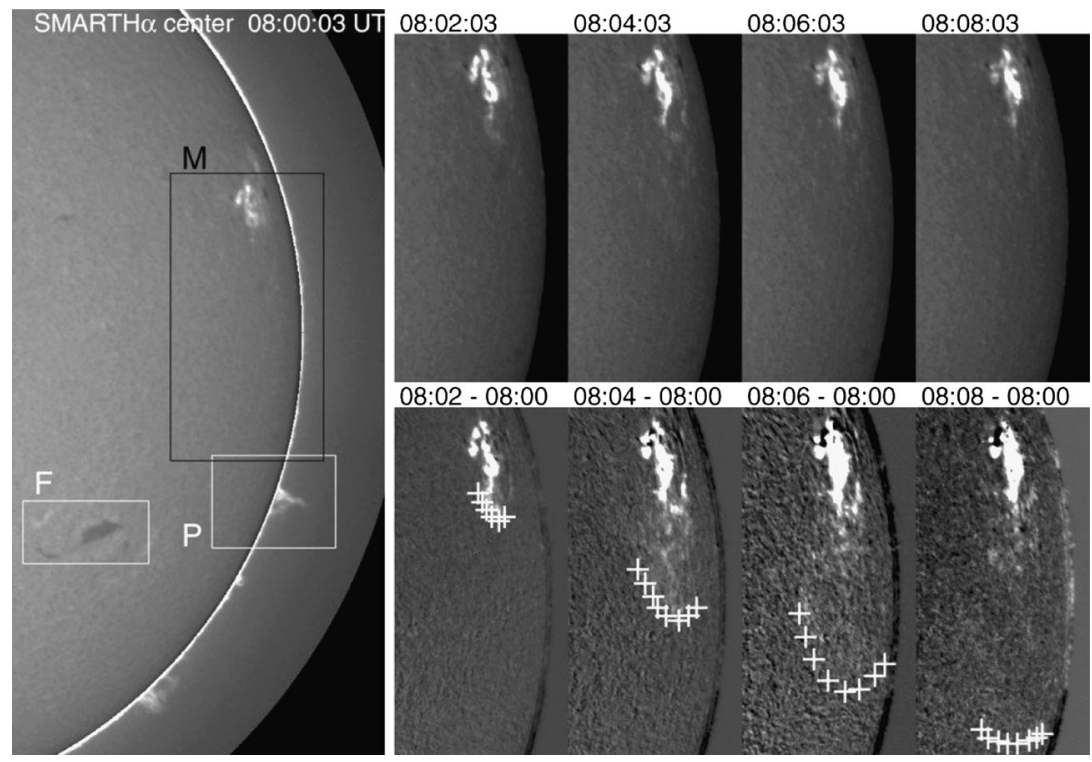

Fig. 4 Example of a Moreton wave observed with the Solar Magnetic Activity Research Telescope (SMART) (http://www.hida.kyoto-u.ac.jp/SMART/). The region "M" in the left panel outlines the region of interest from where a sequence of $\mathrm{H} \alpha$ images are given in normal intensity (top-right panel) and running difference intensity (top-bottom panel). The Moreton wave front is marked by white plus signs. From Asai et al. (2012)

the EIT waves detected in the corona have been regarded as the coronal counterpart of chromospheric Moreton waves. However, several studies showed big discrepancies between Moreton and EIT waves, especially in the estimate of the propagation speed. An example is shown in Fig. 4.

The main features of global EUV waves as deduced from the analysis of numerous observations can be summarised as follows:

- Global coronal waves are mainly seen as a single wave front (a solitary wave).

- They appear to be triggered by impulsive energy releases, such as flares or CMEs. Precise statistical studies show that EUV waves are more associated with CMEs rather than flares. Without CMEs, even a powerful flare cannot produce global waves (Biesecker et al. 2002; Chen 2006). Generally, the appearance of a global coronal wave implies an expanding CME although occasionally the white-light coronagraph may not be able to detect it due to sensitivity. Correspondingly some slow CMEs are not accompanied by clear global coronal waves possibly because the intensity enhancement is too weak.

- EUV waves propagate in the form of a circular or large arc-shaped bright front of the EUV emission, with a typical speed in the range of $200-500 \mathrm{~km} \mathrm{~s}^{-1}$, followed by an expanding dimming region. The dimming is associated with the evacuation of the coronal plasma due to the lift-off of the associated CME flux-rope. Few events reveal a dome-like structure, resulting in a 3D expansion (radial and lateral directions) of the EUV wave (Veronig et al. 2010).

- Kinematic analysis of several events (Fig. 5) show that, after being excited, the wave fronts are drastically decelerated (Veronig et al. 2008; Long et al. 2011) and the magnitude of the deceleration is even higher for waves with higher initial speeds (Warmuth and Mann 2011). 

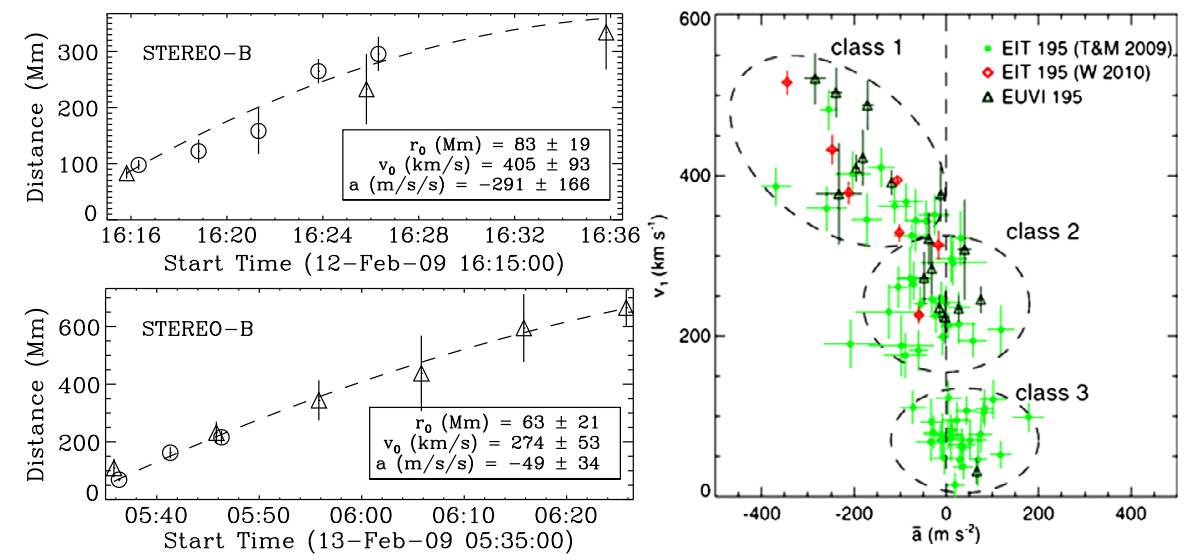

Fig. 5 Left: Plots of distance versus time of the propagating fronts for two EUV waves observed by STERE$\mathrm{O}-\mathrm{B}$. Initial speeds inferred by fitting analysis range between $250-400 \mathrm{~km} \mathrm{~s}^{-1}$, and deceleration of the order of (-290)-(-50) $\mathrm{m} \mathrm{s}^{-2}$. From Long et al. (2011). Right: initial speeds versus acceleration for a large number of EUV events detected with SoHO/EIT and STEREO/EUVI. From Warmuth and Mann (2011)

- The wave fronts can undergo reflection/refraction when they encounter active regions or coronal holes (Gopalswamy et al. 2009) (that are the regions of the decrease or increase in the fast magnetoacoustic speed), or they can stop at magnetic separatrices and appear as stationary bright fronts (Delannée and Aulanier 1999; Attrill et al. 2007).

- Wave fronts are subject to a dispersive evolution, since they tend to broaden their width and attenuate the intensity with the distance (Long et al. 2011).

Based upon these properties, the long-standing debate has led to different physical interpretations and models about the origin of global EUV waves.

\subsection{Theoretical Modelling of Global Coronal Waves}

Two opposite models are considered in order to explain global EUV waves:

- EUV waves are regarded as "true waves", precisely a fast-magnetoacoustic mode propagating mainly in the quiet-Sun region. The launch of EUV waves was proposed by the over-expansion of the CME flanks (Patsourakos and Vourlidas 2009). The phase speed $V_{\mathrm{ph}}$ in linear approximation theory for a fast wave in a homogeneous medium depends on the local sound speed $V_{\mathrm{s}}$ and the local Alfvén speed $V_{\mathrm{A}}: V_{\mathrm{A}}<V_{\mathrm{ph}} \leq V_{\mathrm{f}} \equiv\left(V_{\mathrm{s}}^{2}+V_{\mathrm{A}}^{2}\right)^{1 / 2}$. The estimates from observations of the phase speed, $200-500 \mathrm{~km} \mathrm{~s}^{-1}$, require the coronal plasma- $\beta$ to be close to unity, which is questionable. Comprehensive modelling of EUV waves in terms of non-linear fast waves, based on non-linear geometrical optics, was performed by Afanasyev and Uralov (2011) and gave results consistent with observations.

- EUV waves are "non-waves" or "pseudo-waves", i.e. the simple projection of the CMEs' envelopes over the solar disk, imaged with EUV telescopes because of the heating/compression of the coronal plasma at the surface of the expanding CME flux-rope (Delannée and Aulanier 1999), or due to the result of progressing magnetic restructuring via a series of reconnections between the magnetic field lines of the CME flux-rope and the quiet-Sun region (Attrill et al. 2007).

Each single property of EUV waves, as described previously, supports either one or both of the theories. For example, reflections, deceleration, and dispersive evolution of 

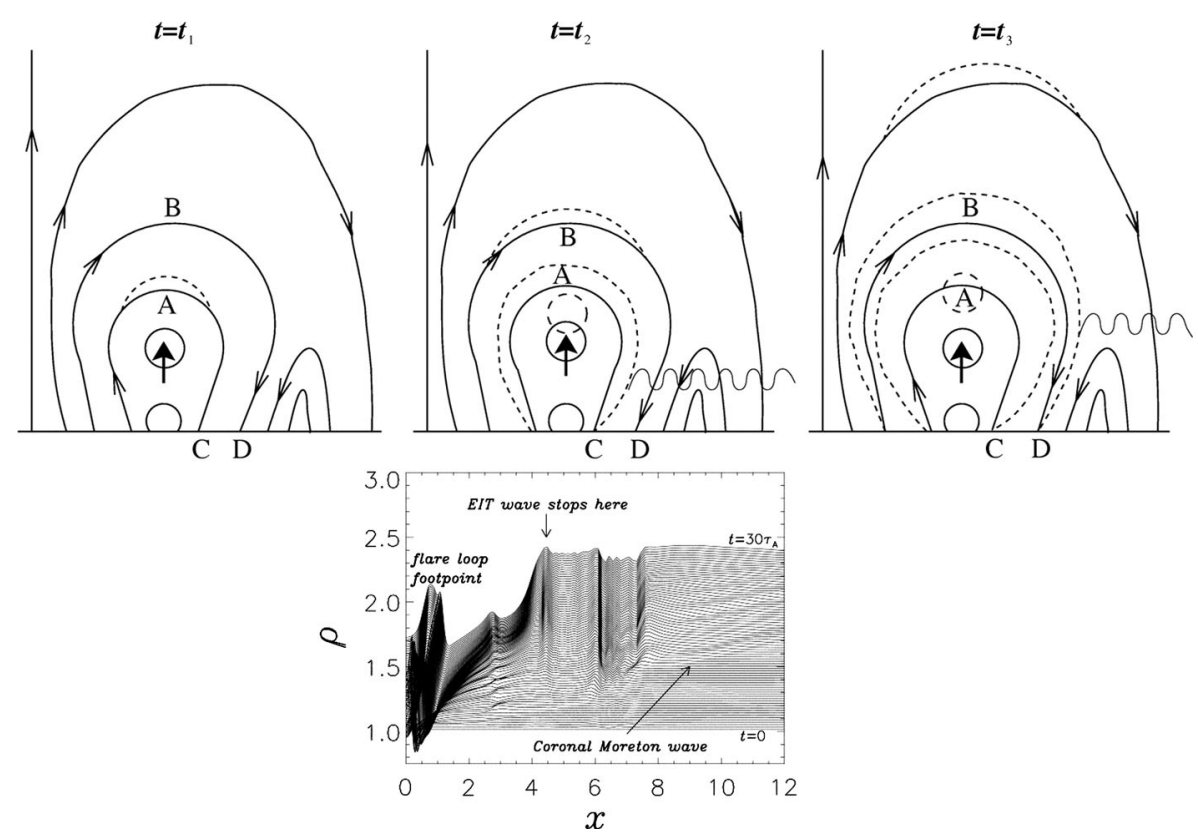

Fig. 6 Upper panels: Mechanism for the formation of global coronal waves. From Chen et al. (2002). Bottom panel: Evolution of the density profiles that initially show the propagation of a coronal Moreton wave from the flare loop footpoint, and then a trailing global coronal (EIT) wave stopping in the vicinity of a small active regions. From Chen et al. (2005)

wave fronts are consistent with the wave theory, whilst brightenings, stoppings at magnetic separatrices of wave fronts, and long-lasting dimmings are better explained in terms of a pseudo-wave scenario. An additional argument to sustain this picture is the estimated phase speed, which is considered too small for being considered typical for fast magnetoacoustic waves.

A third theory combines the previous ones in a "hybrid" model, in order to include the observation of Moreton waves in the chromosphere. The "hybrid" model asserts the existence of two different waves in one single event: first a fast magnetoacoustic shock regarded as the proper coronal counterpart of Moreton waves travelling at a high speed $\left(\sim 10^{3} \mathrm{~km} \mathrm{~s}^{-1}\right)$, then a following pseudo-wave travelling at slower speeds. The mechanism described by Chen et al. $(2002,2005)$ takes into account the propagation of stretching of magnetic field lines in a dipolar region due to the expansion of a CME. The process is described as follows: as the flux-rope rises, the field line near point $\mathrm{A}$ is pushed ahead (i.e. stretching of the field line, see Fig. 6). This perturbation propagates outwards at the fast magnetoacoustic speed. The large-amplitude deformation itself is transferred across the field lines up to $\mathrm{B}$ and also downward reaching point $\mathrm{C}$, where a global coronal wave front appears, in the time interval prescribed by the group velocity of waves. Subsequently, the deformation at point $\mathrm{B}$ is transferred up and also down to point $\mathrm{D}$ with the group velocities of waves. Thus, a new global coronal wave front appears at point $\mathrm{D}$, although the fast magnetoacoustic waves emitted from point $\mathrm{C}$ have already passed point $\mathrm{D}$ travelling at the phase velocity. Therefore, successive stretching of the field lines produces successive density enhancements on the outer side, and global coronal wave fronts are formed causing the evacuation of plasma in the inner region. Simulations (Fig. 6) reveal in detail this behaviour, showing density 
Fig. 7 Response of coronal structures (blue) to different drivers: (a) oscillation from the propagation of a wave (red), which surrounds the $\mathrm{CME}$ flux-rope (black), (b) an expanding CME flux rope pushes outwardly the structures causing bending, (c) the expanding CME magnetically reconnects with the structure, which undergoes an inward jumping. From Patsourakos and Vourlidas (2012)

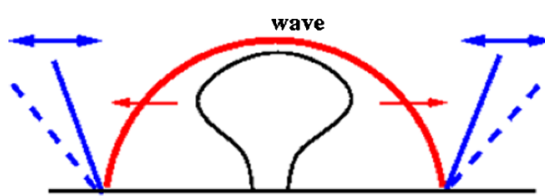

(a) oscillations

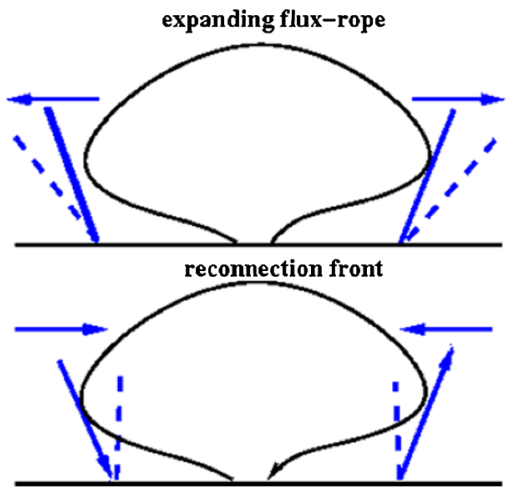

(b) bending

(c) jumping

perturbations associated with the propagation of a fast Moreton wave, and a slower global coronal wave.

Although this model is defined as hybrid, it can be considered as a variant of the pseudowave theory, since the EUV waves observed with the low-cadence telescopes are in any case regarded as "non-true" waves. On the other hand, the recent findings from the Atmospheric Imaging Assembly (AIA) aboard SDO, gave more clues allowing us to detect, in addition to the common EUV wave propagating at hundreds $\mathrm{km} \mathrm{s}^{-1}$, a fast EUV disturbance with a higher speed. This high-speed wave correlates well with chromospheric Moreton waves (Chen and Wu 2011; Asai et al. 2012), providing the key evidence in favour of the hybrid theory. A strong constraint for the validity of the pseudo-wave model is the close and coupled dynamics of the system CME-EUV wave. STEREO observations had a prominent role in determining the $3 \mathrm{D}$ evolution of waves and their relationships with the associated CMEs. Indeed, Fig. 3 shows a time sequence of an EUV-CME event on 13 February 2009 from the STEREO observers, when the spacecraft were separated by $90^{\circ}$ and the source region was simultaneously seen on-disk and off-limb: this gave the rare opportunity to study an EUV wave in quadrature observation, having distinctly a face-on and a lateral perspective. The results show that the EUV wave and the related CME are distinct entities with welldistinct and separated dynamics: an EUV wave is launched by the lateral expansion of the $\mathrm{CME}$, and afterwards it freely propagates through the coronal medium. The interaction of the wave fronts with the medium results in several additional fronts, ripples or wave trains being formed (Patsourakos and Vourlidas 2009; Liu and Ofman 2014), see also Sect. 7.4. Oscillations and deformations of coronal plasma structures, e.g. loops or prominences, after the passage of an EUV disturbance can be explained in terms of the wave transit, as well as an expanding flux rope or reconnection fronts, which cause e.g. their bending or jumping, see Fig. 7 and discussion in Sect. 9.3.

At the present it is not possible to say which mechanism is the most suitable to explain the nature of global coronal waves, because of the large variety of the events, although many elements can be prone to a wave interpretation of the phenomenon. Several questions need to be answered in order to reveal the full nature of the EUV disturbances:

- which parameter determines the formation of the EUV waves (e.g., the impulsiveness of the CME eruption, EUV wave amplitude, etc.); 
- understand better the relationship of EUV waves with powerful flares, type II radio bursts;

- define the 3D evolution of EUV waves and their related CMEs, in particular the physical mechanism for the decoupling of the EUV and CME fronts in the early stages of the evolution;

- the processes occurring during the propagation of EUV in the corona, the response of the coronal medium to the wave transit, quantification of the rate of transmission/reflection through coronal holes, magnetic separatrices and other obstacles;

- deeper understanding of the relationship between global coronal and chromospheric waves;

- determination of the thermal evolution of the EUV waves;

- revealing the effect of fine structuring (the effective dispersion and dissipation) on the wave evolution, including wave scattering and coupling with other modes.

The phenomenon of global coronal waves seems to be similar to impulsive fast magnetoacoustic disturbances propagating in the Earth's magnetosphere, discussed in Sect. 5.4.2. This similarity and its implications need to be studied.

\section{Magnetospheric MHD Waveguides and Resonators}

Natural MHD resonators and waveguides formed by various plasma non-uniformities play an important role in both coronal and magnetospheric physics. Their occurrence causes the effects of wave dispersion, refraction, mode coupling, and the possibility of significant accumulation of the wave energy in certain regions of outer space, where this wave power can influence dynamics of charged particles. Also, an MHD resonator can form a fine multipeak structure of MHD wave spectra, which can be used as a tool for "hydromagnetic spectroscopy" of the space environment, known as MHD seismology. In this section we discuss these phenomena in the Earth's magnetosphere.

\subsection{MHD Waves in a Cold Plasma}

In a cold plasma (i.e. $\beta=0$, a very good approximation for the majority of the magnetosphere), MHD modes can be described by Maxwell's equations with the dielectric permeability being derived via ideal MHD as $\hat{\varepsilon}_{\perp}=c^{2} / V_{\mathrm{A}}^{2}$, where $c$ is the speed of light. For curved field lines it is convenient to use a field-aligned orthogonal curvilinear coordinate system $\left\{x^{1}, x^{2}, x^{3}\right\}$, in which the field lines play the role of coordinate lines $x^{3}$, the stream lines are coordinate lines $x^{2}$, and the surfaces of constant pressure $P$ (magnetic shells) are coordinate surfaces $x^{1}=$ const (Fig. 8). Coordinates $x^{1}$ and $x^{2}$ represent the radial and azimuthal coordinates. The physical length along a field line is expressed in terms of an increment of the corresponding coordinate as $d l_{\|}=\sqrt{g_{3}} d x^{3}$, where $g_{3}$ is the component of the metric tensor, and $\sqrt{g_{3}}$ is the Lamé coefficient denoted as $h_{3}$. Similarly, for the transverse direction we have $d l_{1}=\sqrt{g_{1}} d x^{1}$, and $d l_{2}=\sqrt{g_{2}} d x^{2}$. The corresponding Lamé coefficients are $h_{1}=\sqrt{g_{1}}$ and $h_{2}=\sqrt{g_{2}}$. The determinant of the metric tensor is $g=g_{1} g_{2} g_{3}$.

As any electromagnetic field can be decomposed into the potential and non-potential parts, the MHD wave electric (e) and magnetic (b) fields are decomposed into two modes: the Alfvén mode in which the disturbed magnetic field $\mathbf{b}_{\perp}$ is perpendicular to the equilibrium field $\mathbf{B}_{0}$ and divergence-free, $\nabla \cdot \mathbf{b}_{\perp}=0$, and thus the longitudinal component vanishes, $b_{\|}=0$; and the fast magnetoacoustic mode in which $\mathbf{b}_{\perp}$ is curl-free, $\nabla \times \mathbf{b}_{\perp}=0$, and thus the field-aligned component of the current vanishes, $j_{\|}=0$. The electric field e has only 
Fig. 8 The field-aligned orthogonal curvilinear coordinate system $\left\{x^{1}, x^{2}, x^{3}\right\}$ for the Earth's magnetosphere, in which the field lines play the role of coordinate lines $x^{3}$, the stream lines are azimuthal coordinate lines $x^{2}$, and the magnetic shells are radial coordinate surfaces $x^{1}=$ const. From Mager and Klimushkin (2008)



components transverse to the magnetic field, as $\varepsilon_{\|} \rightarrow \infty$. A 2D electric field $\mathbf{e}_{\perp}$ can be decomposed into the potential (Alfvénic) and solenoidal (magnetoacoustic) parts, characterised by the potentials $\Phi$ and $\Psi$, correspondingly, as

$$
\mathbf{e}_{\perp}=-\nabla_{\perp} \Phi+\nabla_{\perp} \times \mathbf{e}_{\|} \Psi
$$

Thus, linearised MHD equations for waves with the frequencies $\omega$ and wave vector $\mathbf{k}=$ $\left(k_{1}, k_{2}, k_{3}\right.$ ) (where the indices correspond to the axes of the curvilinear coordinate system shown in Fig. 8) in an inhomogeneous plasma immersed into a curvilinear magnetic field can be reduced to the following set

$$
\begin{aligned}
& \hat{L}_{\mathrm{A}} \Phi+\hat{L}_{\mathrm{c}} \Psi=0, \\
& \hat{L}_{\mathrm{F}} \Psi+\hat{L}_{\mathrm{c}}^{+} \Phi=0,
\end{aligned}
$$

where $\hat{L}_{\mathrm{F}}(\omega)$ is the fast magnetoacoustic operator

$$
\hat{L}_{\mathrm{F}}=-\Delta_{\perp} \frac{V_{\mathrm{s}}^{2}+V_{\mathrm{A}}^{2}}{V_{\mathrm{A}}^{2}} \Delta_{\perp}-\left[\partial_{1} \frac{g_{2}}{\sqrt{g}} \hat{L}_{\mathrm{P}} \frac{g_{2}}{\sqrt{g}} \partial_{1}-k_{2}^{2} \frac{g_{1}}{\sqrt{g}} \hat{L}_{\mathrm{T}} \frac{g_{1}}{\sqrt{g}}\right],
$$

with $\Delta_{\perp} \equiv \partial_{1}\left(g_{2} / \sqrt{g}\right) \partial_{1}-k_{2}^{2}\left(g_{1} / \sqrt{g}\right)$, and $V_{\mathrm{s}}$ is the sound speed and $V_{\mathrm{A}}$ is the Alfvén speed; $\hat{L}_{\mathrm{A}}$ is the Alfvénic operator that comprises the Alfvénic toroidal $\hat{L}_{\mathrm{T}}$ and Alfvénic poloidal $\hat{L}_{\mathrm{P}}$ operators,

$$
\hat{L}_{\mathrm{A}}=\partial_{1} \hat{L}_{\mathrm{T}} \partial_{1}-k_{2}^{2} \hat{L}_{\mathrm{P}}
$$

with

$$
\hat{L}_{\mathrm{T}}=\partial_{3} \frac{g_{2}}{\sqrt{g}} \partial_{3}+\frac{\sqrt{g}}{g_{1}} \frac{\omega^{2}}{V_{\mathrm{A}}^{2}}, \quad \hat{L}_{\mathrm{P}}=\partial_{3} \frac{g_{1}}{\sqrt{g}} \partial_{3}+\frac{\sqrt{g}}{g_{2}} \frac{\omega^{2}}{V_{\mathrm{A}}^{2}} ;
$$

and coupling between the modes is determined by the operator $\hat{L}_{\mathrm{c}}$,

$$
\hat{L}_{\mathrm{c}}=i k_{2}\left(\partial_{1} \frac{\omega^{2}}{V_{\mathrm{A}}^{2}}\right)+\left[\partial_{1} \partial_{3} \frac{g_{2}}{\sqrt{g}} \partial_{3} \frac{g_{1}}{\sqrt{g}} i k_{2}-i k_{2} \partial_{3} \frac{g_{1}}{\sqrt{g}} \partial_{3} \frac{g_{2}}{\sqrt{g}} \partial_{1}\right]
$$


and $\hat{L}_{\mathrm{c}}^{+}$is the Hermitian conjugate to the operator $\hat{L}_{\mathrm{c}}$ (Klimushkin 1994). The set of equations (3) was derived under the assumption that the medium is uniform with respect to the azimuthal coordinate $x_{2}$.

The Alfvén and fast magnetoacoustic modes can be decoupled in an inhomogeneous plasma, that is $\hat{L}_{c}=0$, for axially-symmetric disturbances $k_{2} \rightarrow 0$. In the magnetospheric physics the azimuthal wave structure is commonly characterised by the azimuthal wave number $m$, related to the wave vector $k_{2}$ as $k_{2}=m / L_{\mathrm{dis}} R_{\mathrm{E}}$, where $L_{\mathrm{dis}}$ is the dimensionless radial distance in Earth's radii, $r=L_{\mathrm{dis}} R_{\mathrm{E}}$.

In a homogeneous plasma, wave disturbances can be presented as harmonic waves $\propto$ $\exp \left(-i \omega t+i k_{\|} s+i \mathbf{k}_{\perp} \mathbf{r}\right)$, where $s$ is the coordinate along the field, $\mathbf{k}_{\perp}=\left\{k_{x}, k_{y}\right\}$ is the transverse wave number, and $k_{\|}$is the field-aligned component of the wave vector, assuming the equilibrium field directed along the $z$ axis. If a cold plasma is assumed (the sound speed $V_{\mathrm{s}}=0$ ), the general equations (3) are reduced to the dispersion relations for decoupled Alfvén and fast magnetoacoustic modes

$$
\omega_{\mathrm{A}}^{2}=k_{\|}^{2} V_{\mathrm{A}}^{2}, \quad \omega_{\mathrm{F}}^{2}=\left(k_{\|}^{2}+k_{\perp}^{2}\right) V_{\mathrm{A}}^{2},
$$

respectively. The slow magnetoacoustic mode is absent as the plasma is taken as cold, with $V_{\mathrm{s}}=0$.

\subsection{Magnetospheric Cavities and Waveguides for the Fast Magnetoacoustic Mode}

There is a possibility for the magnetosphere to oscillate wholly (or partially) as a collective cavity mode. Owing to this, the magnetosphere should behave as a kind of an MHD FabriPerrot resonator, absorbing the energy from the turbulent solar wind most effectively at the frequencies prescribed by the parameters of the cavity.

In a simple case of a $1 \mathrm{D}$ cold $\left(V_{\mathrm{s}}=0\right)$ plasma inhomogeneous along $\mathrm{x}$, where the Alfvén speed depends only on the radial coordinate, $V_{\mathrm{A}}=V_{\mathrm{A}}(x)$, the fast magnetoacoustic mode dispersion relation given by Eq. (6) may be considered as an equation for the wave vector radial component

$$
k_{x}^{2}(x)=k_{\mathrm{A}}^{2}-k_{\|}^{2}-k_{y}^{2},
$$

where $k_{\mathrm{A}}=\omega / V_{\mathrm{A}}$ is the Alfvén wave number. As follows from this equation, the transparent region where $k_{x}^{2}>0$ for the fixed wave parameters is situated in the regions with a low Alfvén speed, whereas the point $k_{x}^{2}=0$ is the reflection (turning) point for the fast magnetoacoustic mode. Figure 9 shows the Alfvén speed in the magnetosphere. As in the cold plasma the fast speed coincides with the Alfvén speed, the regions with the Alfvén speed minima (cavities) act as natural resonators or waveguides for fast waves. Solutions to a relevant eigenvalue problem give resonant frequencies of the cavities, and the spatial structure of the corresponding fast magnetoacoustic modes.

The cavity oscillations of the entire plasmasphere are considered as a mechanism for global Pi2 waves at nightside (see Keiling and Takahashi 2011, for a review). On the dayside of the magnetosphere, the lowest Alfvén speed region is located near the magnetopause, and the magnetopause is considered as the outer boundary of this resonator. In the azimuthal direction the magnetosphere may be considered as an unbounded system with a gradual transition to the magnetic tail on the nightside. Thus, the eigenmodes of the dayside fast mode resonator can run into the tail. For this reason, it would be more appropriate to call this resonator an open waveguide. The group velocity in the azimuthal direction of a fast mode trapped in this waveguide can be estimated as $V_{\mathrm{gr}}=k_{y} V_{\mathrm{A}}^{2} / \omega(k)$ (Wright 1994). Furthermore, small-scale disturbances with $k_{y} L_{\text {shell }} R_{\mathrm{E}} \gg 1$, where $L_{\text {shell }}$ is the distance from the 
Fig. 9 A sketch of Alfvén speed distribution in the meridional (top panel) and equatorial (bottom panel) sections of the magnetosphere. Areas with different values of the Alfvén speed $\left(A=V_{\mathrm{A}}\right)$ are shown by varying shades of grey. From Mazur and Leonovich (2006)

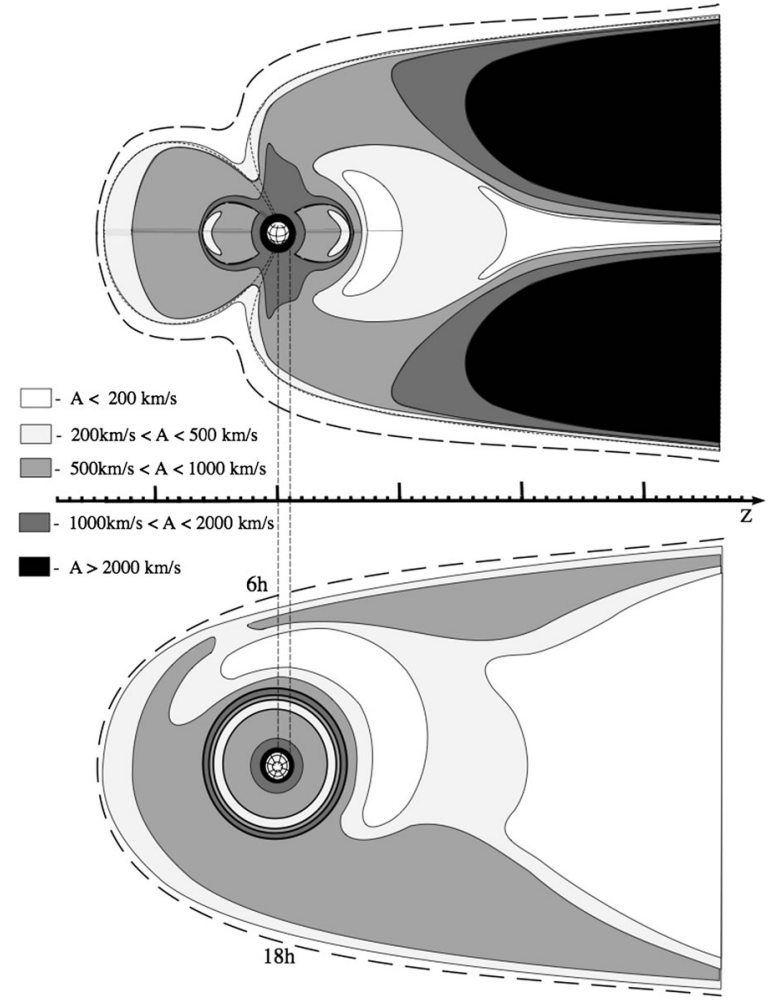

Earth's centre to the magnetic shell (the top of field line), measured in the Earth's radii $R_{\mathrm{E}}$, are convected rapidly at the velocity $V_{\mathrm{gr}} \simeq V_{\mathrm{A}}$ into the magnetotail, whereas large-scale disturbances with $k_{y} L_{\text {shell }} R_{\mathrm{E}} \simeq 1$ and $V_{\mathrm{gr}} \ll V_{\mathrm{A}}$ remain for a longer time in the MHD waveguide and grow to high amplitudes. Thus, the waveguide dispersion results in the selection of modes with small $m$ numbers. The frequency of these modes is determined by the quantisation condition $k_{x} L_{x} \simeq \pi n$, where $L_{x}$ is the typical radial scale of the MHD waveguide. Estimates of the fundamental quarter-wavelength mode with the period $P \approx 4 L_{x} / V_{\mathrm{A}}$, where $L_{x}$ is the size of the waveguide in the direction across the magnetic shells, match the period of global Pc5 pulsations (about $6 \mathrm{~min}$ ), assuming $L_{x} \approx 5 R_{\mathrm{E}}$ and $V_{\mathrm{A}} \approx 400 \mathrm{~km} \mathrm{~s}^{-1}$.

As empirical evidence of this dayside fast magnetoacoustic waveguide, the so-called "magic frequencies" are often considered (Samson et al. 1992). These frequencies constitute a preferred set for peaks in the spectrum of the Alfvén oscillations observed on the ground. Since those Alfvén oscillations are considered as excited by the fast magnetoacoustic modes on the magnetospheric lobe in the course of the resonant conversion (see Sect. 5), their spectrum must reveal the spectrum of their sources. The magnetoacoustic eigenmodes of this resonator can also be generated by some processes in the solar wind, as discussed in Sect. 9.1.

Another low Alfvén speed region is located in the near-Earth plasma layer in the nightside magnetosphere (Mazur and Leonovich 2006; Leonovich and Mazur 2008). The corresponding fast magnetoacoustic waveguide can be responsible for the lowest frequency part of the spectrum of the "magic frequencies". Another minimum of the Alfvén speed is situated just inside the plasmapause. The corresponding small scale fast magnetoacoustic eigen oscilla- 
Fig. 10 Azimuthal structure and field line oscillation in the toroidal $(m=3)$ and poloidal $(m=70)$ Alfvén modes

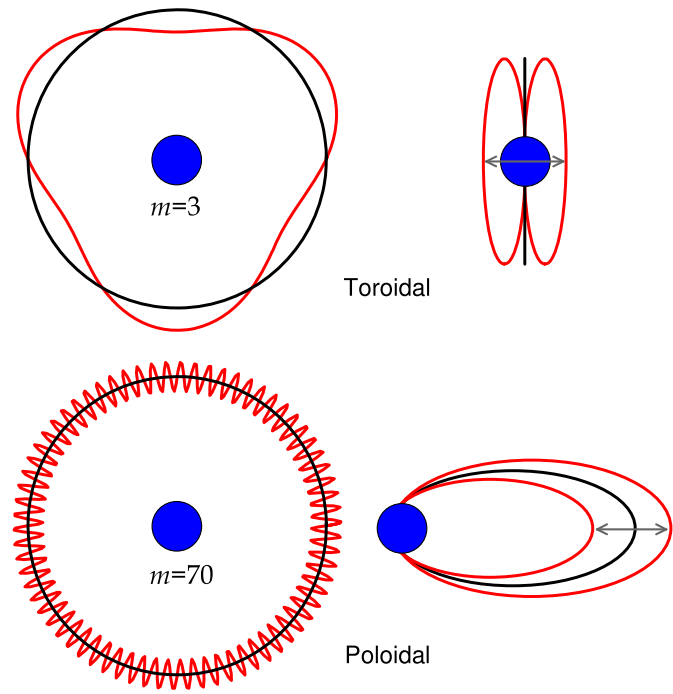

tions can be responsible for some Pc3 waves (Gul'elmi 1970), although the corresponding resonator has rather low quality (Leonovich and Mazur 2001).

\subsection{Magnetospheric Alfvén Resonator}

In the direction along the closed field lines, the magnetosphere is bound by the highly conductive ionosphere. In the simplest model of the magnetosphere the field lines can be assumed to be straight with a constant Alfvén speed. Then the parallel wave vector component $k_{\|}$in the Alfvén mode dispersion relation described by Eq. (6) must be quantised, $k_{\|}=2 \pi / N S_{\mathrm{fl}}$, where $N$ is an integer representing the parallel harmonic wave number and $S_{\mathrm{fl}}$ is the field line length between the conjugate ionosphere. Consequently, the Alfvén eigenfrequency is $\omega_{\mathrm{AN}}=(2 \pi / N) V_{\mathrm{A}} / S_{\mathrm{fl}}$. This phenomenon is known as the field line resonance (FLR).

In a curved magnetic field, the spectral theory of MHD resonators predicts that the complete spectrum of Alfvénic-type eigenoscillations comprises of two sets of non-local dispersion equations. These dispersion equations correspond to the toroidal mode with small azimuthal wave numbers $m \simeq 1$ and poloidal mode with large $m$ numbers $m \gg 1$. The azimuthal structure of those two modes is illustrated in Fig. 10. Azimuthally largescale toroidal Alfvén waves are predominantly excited by large-scale extra-magnetospheric sources and effectively penetrate down to the ground (Pc3-5 and $\mathrm{Pi} 2-3$ pulsations). Poloidal Alfvén waves are mostly generated by local intra-magnetospheric sources (storm-time Pc5 pulsations, and Pg pulsations), but these small-scale waves are nearly completely screened by the ionosphere from ground magnetometers.

Magnetospheric toroidal modes are Alfvén waves with large azimuthal scales, so $\left|k_{1}\right| \approx$ $\left|\partial_{1}\right| \gg\left|k_{2}\right|$. They are characterised by the large radial electric $e_{1}$ and azimuthal magnetic $b_{2}$ field. Their field-aligned spatial structure is governed by the ordinary differential equation

$$
\hat{L}_{\mathrm{T}}(\omega) e_{1}=0 \text {. }
$$

Poloidal modes are Alfvén waves with small azimuthal scales, so $\left|k_{1}\right| \approx\left|\partial_{1}\right| \ll\left|k_{2}\right|$. They have large azimuthal electric $e_{2}$ and radial magnetic $b_{1}$ fields. Their parallel structure is 
governed by the ordinary differential equation

$$
\hat{L}_{\mathrm{P}}(\omega) e_{2}=0 \text {. }
$$

As these two modes are described by different differential equations, they also have different eigenfrequencies, $\omega_{\mathrm{TN}}$ (the toroidal eigenfrequency) and $\omega_{\mathrm{PN}}$ (the poloidal eigenfrequency), respectively. This difference is referred to as the polarisation splitting of the spectrum. In the leading order of the WKB approximation along the parallel coordinate, the eigenfrequencies of these two modes are almost identical:

$$
\omega_{\mathrm{TN}} \approx \omega_{\mathrm{PN}} \approx \frac{2 \pi N}{\tau_{\mathrm{A}}}
$$

where $\tau_{\mathrm{A}}=\oint d s / V_{\mathrm{A}}$ is the travel time along the field line at the local Alfvén speed in both directions. In the dipole model, the toroidal eigenfrequency is larger than the poloidal eigenfrequency: $\omega_{\mathrm{TN}}>\omega_{\mathrm{PN}}$. For the fundamental harmonic $(N=1)$, it is about 1.5 times larger, while for higher harmonics this difference is much smaller.

\subsection{Alfvén Waves in a Finite-Pressure Plasma}

Another factor influencing the polarisation splitting of Alfvénic modes is the plasma pressure (also referred to as finite- $\beta$ effects). Besides the Ampère force, the finite- $\beta$ plasma is subjected to the hydrodynamic force $\nabla p$, where $p$ is the gas pressure. The pressure perturbation $\tilde{p}$ can be expressed as

$$
\tilde{p}=-\boldsymbol{\xi} \cdot \nabla p_{0}-\gamma_{0} p_{0} \nabla \cdot \boldsymbol{\xi},
$$

where $\boldsymbol{\xi}$ is the displacement of the plasma from the equilibrium position, $p_{0}$ is the equilibrium gas pressure, and $\gamma_{0}$ is the adiabatic index. In an axisymmetric model the plasma pressure depends only on the radial coordinate, thus the first term of the right hand side here is not zero for the poloidal mode in which the plasma is displaced in the radial direction. The second term demands additional consideration. For the Alfvén wave in a homogeneous plasma, the displacement has a solenoidal character, $\nabla \cdot \xi=0$. However, for plasma embedded in a curved magnetic field the situation is different. If the plasma is displaced in the radial direction, on the normal to the curved field lines, then due to purely geometrical reasons the plasma will be compressed, and pressure perturbations will appear (Southwood and Saunders 1985). For these two reasons, the radial force acting on a warm plasma will be different to that of a cold plasma. This effect may be considered as linear coupling of the true, incompressive Alfvén waves with compressive slow magnetoacoustic waves, caused by the magnetic field curvature. Direct observational evidence of this coupling in the magnetotail was recently found by Du et al. (2011).

The different force for the radial displacement also results in a different wave frequency. In the toroidal mode the plasma is displaced in the azimuthal direction, simply sliding along the magnetic shells, and hence there is no additional force compared with the cold plasma case. So the field-aligned structure of the toroidal mode is determined from the same Eq. (8) as for a cold plasma, and the toroidal frequency will be unaltered. However, Eq. (9) for the poloidal mode field aligned structure is substituted for the equation (Denton 1998; Mager and Klimushkin 2002; Klimushkin et al. 2004),

$$
\left[\partial_{3} \frac{g_{1}}{\sqrt{g}} \partial_{3}+\frac{\sqrt{g}}{g_{2}}\left(k_{\mathrm{A}}^{2}+\eta_{\beta}\right)\right] e_{2}=0,
$$


where the term

$$
\eta_{\beta}=-\frac{2}{R}\left(\frac{4 \pi p^{\prime}}{B^{2}}+\frac{2}{R} \frac{4 \pi \gamma_{0} p}{B^{2}}\right)
$$

is called the ballooning term, where $R$ represents the field line curvature. Equation (12) corresponds to the dispersion relation

$$
k_{\|}^{2}=k_{\mathrm{A}}^{2}-\eta_{\beta} .
$$

In the WKB approximation, the poloidal frequency $\omega_{\mathrm{PN}}$ becomes

$$
\omega_{\mathrm{PN}}=\frac{2 \pi N}{\tau_{\mathrm{A}}}-\frac{1}{4 \pi N} \oint V_{\mathrm{A}} \eta_{\beta} d s .
$$

The sign of the difference $\left(\omega_{\mathrm{TN}}-\omega_{\mathrm{PN}}\right)$ depends on the plasma pressure distribution, while it is always positive in a cold plasma. There are also other factors that may influence the polarisation splitting of the spectral peaks, such as the rotation of the magnetospheric plasma (Kozlov and Leonovich 2006) or the magnetic shear caused by field-aligned currents (Klimushkin and Mager 2004).

Another interesting consequence of the finite plasma pressure is its compressional character. In a homogeneous plasma, the shear Alfvén mode does not perturb the parallel magnetic field. However, in a hot plasma embedded in a curved magnetic field the poloidal Alfvén mode (the linearly polarised Alfvén wave with the perturbation of the magnetic field in the same plane with the radius of the curvature of the equilibrium field) causes the plasma compression. In its turn, the plasma compression causes the plasma pressure perturbation $\tilde{p}$. For the ULF mode with frequency $\omega \ll k_{\perp} V_{\mathrm{A}}$, the sum of the plasma and magnetic pressure perturbations is close to zero,

$$
\tilde{p}+\frac{B b_{\|}}{4 \pi}=0
$$

(e.g., Pokhotelov et al. 2000). It presumes a nonzero compressional magnetic field component. The relation of the parallel and transverse magnetic field components of an Alfvén wave can be estimated as

$$
\frac{b_{\|}}{b_{r}} \approx \frac{\beta}{k_{\|} L_{\perp}},
$$

where $b_{r}$ is the perturbation of the magnetic field along the radius of the curvature of the field, and $L_{\perp}$ is the characteristic scale of transverse non-uniformity. Thus, the observed compressional component of the poloidal ULF wave evidences the role of the plasma pressure in the wave formation.

\subsection{Alfvénic Resonators on Open Field Lines}

In some situations local Alfvénic resonators can be formed on sections of field lines, without any reflective boundaries, e.g., in the high latitude magnetosphere. The high latitudes are of special importance for space geophysics. Due to the topology of the near-Earth magnetic field the high latitudes are conjugate to the boundary layers of the magnetosphere, where the interaction of the solar wind flow with the magnetosphere occurs. This interaction has a non-steady and turbulent character, so the high-latitude ULF transients are indicators of such interaction. However, at high latitudes, where field lines are open or strongly extended 
into the magnetotail, many previously devised notions on Alfvén resonators for closed field lines between the conjugated ionospheres, must be revisited.

A sharp local change in the geometry of the background magnetic field may result in a partial reflection of Alfvén waves. This effect can lead to the formation of quasi-resonators on open field lines even in the absence of conducting ends or sharp plasma density gradients (Mazur et al. 2004). A high "sensitivity" of Alfvén waves to the magnetic field geometry may be highlighted by presenting equations for toroidal and poloidal modes via maximum and minimum curvature of the surfaces orthogonal to field lines, $K_{+}$and $K_{-}$:

$$
\left[\frac{\partial^{2}}{\partial s^{2}}+\left(K_{+}-K_{-}\right) \frac{\partial}{\partial s}+k_{\mathrm{A}}^{2}\right] e_{1}=0, \quad\left[\frac{\partial^{2}}{\partial s^{2}}-\left(K_{+}-K_{-}\right) \frac{\partial}{\partial s}+k_{\mathrm{A}}^{2}\right] e_{2}=0,
$$

where $s$ is the length along the field line, and $d s=h_{3} d x^{3}$.

It is convenient to rewrite Eqs. (8) and (9) in a form similar to the equation for Alfvén waves in a uniform magnetic field. The replacement $d \zeta=h_{1}^{2} d x^{3}$ and $d \eta=h_{2}^{2} d x^{3}$, reduces them to

$$
\left[\partial_{\zeta \zeta}+\frac{\omega^{2}}{V_{\mathrm{T}}^{2}}\right] e_{1}=0, \quad\left[\partial_{\eta \eta}+\frac{\omega^{2}}{V_{\mathrm{P}}^{2}}\right] e_{2}=0 .
$$

Here $V_{\mathrm{T}}$ and $V_{\mathrm{P}}$ are the modified Alfvén velocities determined as

$$
V_{\mathrm{T}}=\frac{h_{1}}{h_{2}} V_{A}=\frac{1}{h_{2}^{2} \sqrt{4 \pi \rho}}, \quad V_{\mathrm{P}}=\frac{h_{2}}{h_{1}} V_{A}=\frac{1}{h_{1}^{2} \sqrt{4 \pi \rho}} .
$$

Thus, the propagation of both toroidal and poloidal Alfvén modes in a curvilinear magnetic field can be described by equations similar to the equation in a straight field with the Alfvén speed $V_{\mathrm{A}}$ replaced with the modified Alfvén velocities $V_{\mathrm{T}}$ and $V_{\mathrm{P}}$, respectively.

As illustration, let us consider the propagation of Alfvén waves in 2D plane-parallel magnetic field geometry $\mathbf{B}_{0}(x, z)$. Let Alfvén waves propagate along field lines and then impinge into a region where the field line geometry changes sharply, e.g., field lines are rapidly bending and converging. In the absence of a density gradient ( $\rho=$ const), the toroidal mode does not "feel" the magnetic field curvature. In contrast, for a poloidal mode in a curvilinear magnetic field, the effective Alfvén velocity $V_{\mathrm{P}}$ is highly non-uniform along the parallel coordinate $s$ even in the case of a constant plasma density, because the Lamé coefficient strongly depends on this coordinate.

Analytical estimates of the influence of the field line curvature on propagation of Alfvén waves can be done only in a simplified geometry, where the Lamé coefficients are known functions. As an example, a superposition of a uniform magnetic field $B_{0} \hat{\mathbf{z}}$ and the field produced by a linear current with the density $\mathbf{j}_{0}=-I_{0} \delta(x) \delta(z) \hat{\mathbf{y}}$ can be considered. The characteristic scale length $r_{0}=\mu_{0} I_{0}\left(2 \pi B_{0}\right)^{-1}$ in this system corresponds to the distance at which the field of the linear current becomes comparable to the uniform field. At $|x|,|z| \leq$ $r_{0}$, field lines undergo a break and rapidly converge or diverge near the current. How do these regions affect the propagation of Alfvén waves? The specific features of wave propagation can be quantitatively characterised by the coefficient of reflection from a barrier arising due to the convergence and divergence of the field lines.

Figure 11 (left-hand panel) shows typical field lines passing through the regions where the field lines converge $(x \geq 0)$ and diverge $\left(x \leq-r_{0}\right)$. The calculated dependences of the absolute value of the reflection coefficient $|R(\bar{\omega})|$ for the same field lines are shown in Fig. 11 (right-hand panel). The normalised frequency $\bar{\omega}=\omega r_{0} / V_{\mathrm{A}}^{(\infty)}$ is determined by the 
Fig. 11 Left-hand panel: 2D magnetic field configuration. Right-hand panel: Reflection coefficients of poloidal Alfvén modes propagating along the curved field lines. The curves $|R(\Omega)|$ and the corresponding field lines are shown by the same type of line: heavy, light, dashed, and dashed-and-dotted, for diverging field lines
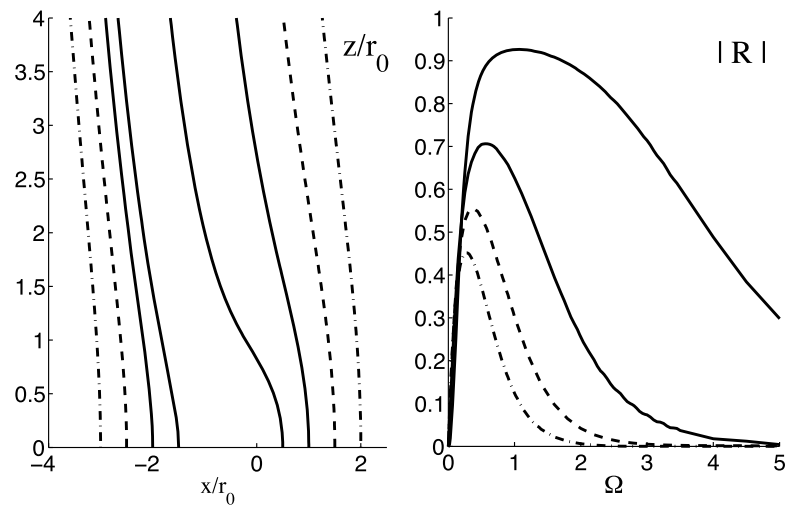

non-uniformity scale $r_{0}$ and the Alfvén velocity at infinity $V_{\mathrm{A}}^{(\infty)}$. This plot shows that the reflection of the Alfvén wave is fairly large, up to $80-90 \%$. The dependence $R(\bar{\omega})$ is nonmonotonic. For high-frequency waves $(\bar{\omega} \gg 1)$, the reflection coefficient is small, which is quite natural for waves that can be described in the WKB approximation. In the range of very low frequencies, which, in fact, corresponds to the case of nearly DC perturbations, the reflection coefficient also tends to zero, $R \rightarrow 0$. The reflection of an Alfvén wave is most effective when the wavelength is comparable to the non-uniformity scale length $r_{0}$, that is $\bar{\omega} \leq 1$.

This consideration shows that the propagation of Alfvén waves can be significantly affected by local variations in the geometry of the magnetic field. For example, magnetospheric field lines emerging from the night-time high-latitude ionosphere undergo a sharp break and rapidly converge in the current sheet of the geomagnetic tail. In this region quasiperiodic oscillations can be excited, with the periods determined by the size of the Alfvén quasi-resonator between the ionosphere and current sheet.

Thus, even in a system without reflecting boundaries or steep plasma gradients the formation of an Alfvénic quasi-resonator on open field lines becomes possible. Poloidal Alfvén waves would be most sensitive to the geometry of magnetic field lines. The wave partial reflection from a region with a localised steep change of magnetic geometry (e.g. "bending", "compression" or "rarefaction" of field flux tubes) is a merely geometrical effect, formally caused by the dependence of the Lamé coefficients on the field-aligned coordinate. Similar situations may occur in space and laboratory plasmas. In the solar atmosphere, formation of coronal loop resonators for torsional Alfvén waves and penetration of Alfvén waves into the corona depend critically on reflection at the loop ends. The variation of the magnetic field flux tube cross-section with height may increase this reflection substantially.

In the solar corona, the role of the field-aligned non-uniformity of the Alfvén speed in the propagation of coronal waves has been intensively studied since the seminal paper of Ferraro and Plumpton (1958). Consider an isothermal stratified atmosphere with a uniform vertical magnetic field, with the equilibrium density $\rho_{0} \propto \exp \left(-z / H_{\mathrm{g}}\right)$ and the Alfvén speed $V_{\mathrm{A}} \propto \exp \left(z / 2 H_{\mathrm{g}}\right)$, where $H_{\mathrm{g}}$ is the gravitational scale height determined by the plasma temperature. In such a medium, the linearly polarised Alfvén wave is described by the expressions

$$
\begin{aligned}
& V_{x}(z, t)=\left[A_{1} J_{0}\left(\omega / \omega_{\mathrm{Ag}}(z)\right)+A_{2} Y_{0}\left(\omega / \omega_{\mathrm{Ag}}(z)\right)\right] \exp (i \omega t), \\
& B_{x}(z, t)=\rho_{0}^{1 / 2}(z)\left[A_{3} J_{1}\left(\omega / \omega_{\mathrm{Ag}}(z)\right)+A_{4} Y_{1}\left(\omega / \omega_{\mathrm{Ag}}(z)\right)\right] \exp (i \omega t),
\end{aligned}
$$


Fig. 12 Field-aligned structure of the fundamental harmonic in the vicinity of the magnetospheric equatorial plane $(s=0)$. Shown are the wave potential $\Phi(s)(\beta>0$ case $)$ and magnetic field $b_{1}(s)(\beta=0$ and $\beta>0$ cases). From Mager et al. (2009)
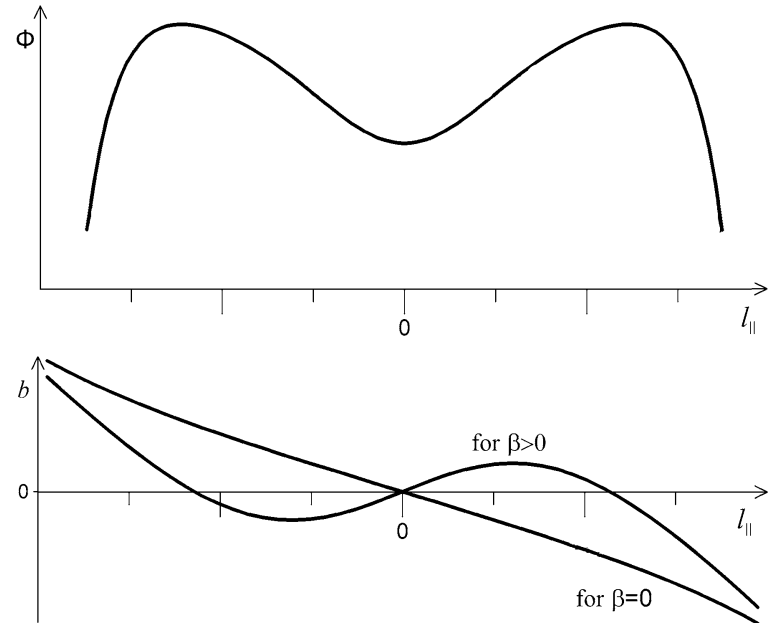

where $J_{0}$ and $J_{1}$ are the Bessel functions of the first kind, and $Y_{0}$ and $Y_{1}$ are the Bessel functions of the second kind, of the first and second order, respectively, $A_{1}, A_{2}, A_{3}$ and $A_{4}$ are arbitrary constants determined by boundary conditions, and $\omega_{\mathrm{Ag}}(z)=V_{\mathrm{A}}(z) / 2 H_{\mathrm{g}}$. In particular, for a wave propagating in the positive $z$ direction, $A_{2}=A_{4}=0$. The Alfvén speed variation along the magnetic field leads to the wave reflection, which is intensively studied theoretically in the context of the solar wind acceleration and heating (e.g. Cranmer and van Ballegooijen 2005; Ofman 2010).

\subsection{Alfvénic Field Line Resonators in a Warm Plasma}

Another cause of Alfvénic resonators along field lines is finite plasma pressure. Indeed, in dispersion relation (14) the first term in $\eta_{\beta}$ (see Eq. 13 ) is usually smaller than the second one. In a plasma region with $\beta \sim 1$ (e.g., on the magnetic equator), the situation is possible when an opaque region (where $k_{\|}^{2}<0$ ) for the poloidal Alfvén wave can be formed. This region is bounded by two turning points $\left(k_{\|}^{2}=0\right)$ which restrain penetration of the wave energy, and an Alfvén resonator appears on a part of the field line (Mager et al. 2009). Due to this effect the magnetospheric ULF pulsations in the Northern and Southern hemispheres at very high latitudes can be non-conjugated. If the wave is generated on the whole field line, it must be composed of two weakly related parts adjacent to the ionosphere with an amplitude dip in the equatorial region (Fig. 12).

\subsection{Alfvén Resonators Across the Field Lines}

In a homogeneous plasma, the Alfvén mode dispersion relation does not include terms related to the wave's structure across the field line. However, in a curved magnetic field, the frequencies of the oscillations polarised in the radial and azimuthal direction become different: $\omega \rightarrow \omega_{\mathrm{TN}}$ at $k_{r} \rightarrow \infty$ and $\omega \rightarrow \omega_{\mathrm{PN}}$ at $k_{r} \rightarrow 0$. This dependence can be formulated as

$$
k_{r}^{2}=-k_{\mathrm{A}}^{2} \frac{\omega^{2}-\omega_{\mathrm{PN}}^{2}}{\omega^{2}-\omega_{\mathrm{TN}}^{2}}
$$

(Klimushkin et al. 2004). The point where $k_{r}(r)=0$ is referred to as the turning point, or cut-off shell. As is seen from this equation, it is situated on the magnetic shell where 
the wave's frequency $\omega$ equals the poloidal eigenfrequency $\omega_{\mathrm{PN}}$. In this approximation the waves are weakly compressive.

In an inhomogeneous plasma, the poloidal frequency $\omega_{\mathrm{PN}}(r)$ as a function of the radial coordinate $r$ can have extremes. In this case the Alfvén mode is bounded on both sides by the cut-off shells, where $k_{r}=0$. Between these shells the wave energy is trapped in the direction transverse to the magnetic surfaces. A trapped wave is channelled along the azimuth, being a standing wave in both field aligned and radial directions (Leonovich and Mazur 1995b; Klimushkin 1998).

Whether such a resonator is situated at the minima or maxima of the cut-off frequency depends on the sign of the difference between the resonance and cut-off frequency $\left(\omega_{\mathrm{TN}}-\omega_{\mathrm{PN}}\right)$. If this difference is positive, then the resonator is situated near the minima of the function $\omega_{\mathrm{PN}}(x)$; in the opposite case, it is situated near the maxima (Klimushkin 1998). The first case can occur near the ring current maximum, while the second one may take place on the outer edge of the plasmapause.

The wave polarisation in the resonator must be close to poloidal. The radial structure of the principal radial harmonic as a function of the radial coordinate is described by a Gaussian function. The resonator modes have a discrete spectrum determined from the quantisation condition: the number of radial half-waves between the poloidal surfaces should be an integer. The dependence of the wave vector radial component on the frequency $\omega$ leads to frequency quantisation: the Alfvén wave acquires the discrete coordinate-independent spectrum.

Satellite and radar studies reported that poloidal high- $m$ Pc4-5 waves often have a narrow localisation across magnetic shells with a period constant in the radial direction (Schäfer et al. 2008; Takahashi et al. 2011). Observed poloidal oscillations may represent modes of the transverse resonators. According to the ground magnetometer data, the same behaviour is typical for the so called giant pulsations, a subclass of the poloidal Pc4 pulsations with moderate azimuthal wave numbers $(m \simeq 15)$. One can suppose that these pulsations are the modes of a resonator on the outer boundary of the plasmapause (Mager and Klimushkin 2013).

The observed properties of the wave in the resonator should depend on the way of its excitation. If it is induced by some impulsive source, the wave cannot remain in the pure poloidal polarisation. The wave field in the resonator is the superposition of many harmonics with different radial structures and eigenfrequencies. Such ensemble of the superposed transverse harmonics must result in the amplitude modulation (Mager and Klimushkin 2013). The phase fronts in the impulsively excited resonator must be curved. This behaviour was observed with SuperDARN radars in several cases of poloidal waves (Yeoman et al. 2012).

\subsection{Resonators and Waveguides in the Earth's Ionosphere and Atmosphere}

Electromagnetic waves can propagate in an atmospheric waveguide bounded by conducting ionosphere and the conducting ground. As a first approximation, the atmosphere can be considered to be a perfect insulator between perfectly conducting boundaries, separated by a height $h$. The distinct wave solutions of Maxwell's equations in vacuum are the magnetic (or transverse electric TE) mode, containing the vertical $b_{z}$ component, and an electric (transverse magnetic TM) mode, containing vertical electric $e_{z}$ component. The lowest possible TE mode has dispersion equation $k_{0}^{2}=k_{\perp}^{2}+(\pi / h)^{2}$, where $k_{0}=\omega / c$ is the vacuum wave number, which implies a cut-off frequency of $\pi c / h$. For a nominal ionospheric height $h=100 \mathrm{~km}$ this cutoff frequency is $\sim 1.5 \mathrm{kHz}$, well above the ULF range. Thus, the ULF magnetic mode of such a waveguide is always evanescent. The lowest TM mode has dispersion equation $\omega=k_{\perp} c$, implying a zero frequency cutoff. Thus, the fundamental $\mathrm{TM}_{0}$ mode 
with phase speed $c$ can propagate in the ULF band. However, owing to the high resistance of the atmosphere, the current density near the Earth's surface is much less than the density of ionospheric currents. Therefore, the TM-mode is very poorly excited by magnetospheric disturbances. However it is effectively excited by atmospheric sources such as lightning.

For ULF waves, the Earth's atmosphere is not planar, but rather a spherical shell. This suggests that it acts more like a resonant cavity than a waveguide. This cavity supports Schumann resonances. The resonance frequencies of these modes for perfectly conducting boundaries is given by

$$
\omega=\frac{c}{R_{\mathrm{E}}} \sqrt{l(l+1)},
$$

where $l$ is integer. The fundamental mode $(l=1)$ with frequency about $8 \mathrm{~Hz}$ roughly corresponds to a wave with a wavelength equal to the circumference of the Earth. The Schumann resonator is permanently excited by world-wide thunderstorm activity, so this emission is a ubiquitous feature of the Earth's electromagnetic environment.

\subsubsection{Ionospheric Alfvénic Resonator and Fast Magnetoacoustic Waveguide}

A possibility of the Alfvén resonator along a field line in an unbounded system is non-trivial, because it seemingly contradicts the notion about the absence of turning point for shear Alfvén waves (see also the discussion in Sect. 3.5). It follows from the MHD wave equation for Alfvén waves that these waves have no reflection points. It would seem, therefore, that no field-aligned resonators in which the wave energy could be accumulated may occur for these waves. However, if there are regions with sharp longitudinal variations in the Alfvén speed, then Alfvén waves can be partially reflected from these regions (Leroy 1980). For perturbations with a wavelength comparable to the inhomogeneity scale, the geometricoptics approximation is violated, so that a substantial fraction of the wave energy can be reflected. Such a reflection can lead to the formation of quasi-resonators. The accumulation of wave energy in Alfvén resonators can significantly affect large-scale plasma dynamics.

A peculiar feature of the geomagnetic variations in the ULF band, just below the fundamental tone of Schumann resonance, is the occurrence of multi-band spectral structure, observed at all latitudes. The lowest frequency of this structure is about a fraction of $\mathrm{Hz}$, and the difference between the spectral harmonics is $\Delta f \simeq 0.3-0.5 \mathrm{~Hz}$. The multi-band spectral structure is a typical nighttime phenomenon, and demonstrates a clear anti-correlation with the 11-year solar activity cycle. The occurrence of this spectral structure is attributed to the Ionospheric Alfvén Resonator (IAR) in the upper ionosphere. The occurrence of IAR is evident from the altitudinal profile of the Alfvén refractive index $n_{\mathrm{A}}(s)=c / V_{\mathrm{A}}(s)$ in the ionosphere (Fig. 13). Owing to the partial reflection of the Alfvén waves from the steep gradient of the Alfvén speed vertical profile $V_{\mathrm{A}}(z)$ the upper IAR boundary is formed at an altitude of a few thousands $\mathrm{km}$. The IAR lower boundary coincides with the ionospheric bottom-side boundary. IAR is the example of a system with leaky modes. The idea of the Alfvén resonator originated in solar physics (Leroy 1980), but became widely used in the terrestrial and planetary physics. In particular the IAR formation along an inhomogeneous Jupiter-Io flux tube was suggested for explaining the periodic modulation of the Jovian electromagnetic emission ("S-bursts", Ergun et al. 2006).

The ionospheric cavity with a minimum of $V_{\mathrm{A}}(z)$ (or maximum of $n_{\mathrm{A}}(z)$ ) works not only as a resonator for Alfvén waves, but also as a waveguide for fast magnetoacoustic waves. The guided fast magnetoacoustic modes can propagate to large distances (up to few thousand $\mathrm{km}$ ) along the ionosphere. In the frequency domain ionospheric wave guidance 
Fig. 13 Profiles of the Alfvénic refractive index $n_{\mathrm{A}}(s)$ in the mid-latitude ionosphere for various UT. The upper set of curves $(\mathrm{UT}=0,8,22)$ correspond to daytime hours, the lower set of curves $(\mathrm{UT}=11,16,18)$ correspond to nighttime hours

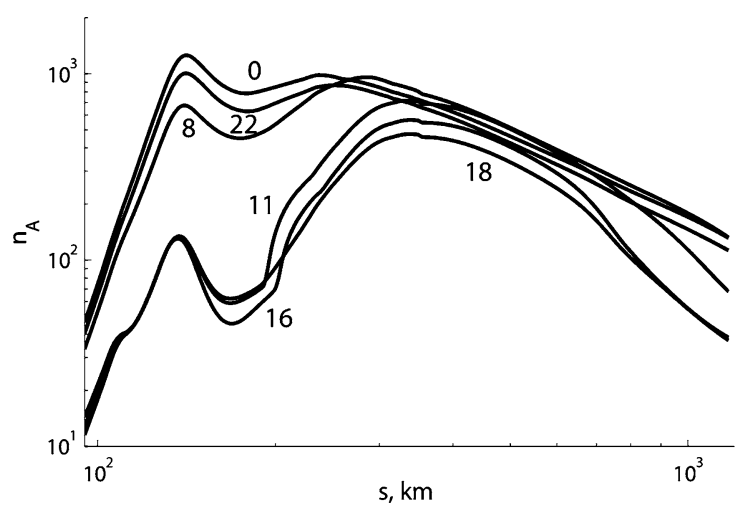

creates a low-frequency cut-off corresponding to the waveguide critical frequency that is lower than the IAR fundamental frequency.

The spectral properties of the IAR have been reproduced by numerous analytical and numerical models. The damping rates and Q-factors (the quality factors, determined as the ratio of the damping time to the oscillation period) of the IAR modes strongly depend on the Alfvén velocity differential in the upper ionosphere and in the lower ionosphere conductivity. More gradual vertical profiles during daytime hours and enhanced conductivity of the lower ionosphere are the main causes of IAR signature suppression during daytime hours and periods of high solar activity.

The IAR in the terrestrial ionosphere can be excited by various mechanisms, comprising of magnetospheric, ionospheric, and atmospheric sources. At auroral latitudes the IAR can be excited by a magnetospheric non-steady field-aligned current, and even may become unstable due to a feedback instability in the coupled magnetosphere-ionosphere system (Lysak 1991; Pokhotelov et al. 2001). A possible energy source for the IAR excitation is related to atmospheric lightning discharges. World thunderstorm centres in the tropics were suggested as the primary source of IAR excitation. As an alternative mechanism, it was suggested that the thunderstorms occurring nearby are able to generate the signals in the IAR range with sufficient intensities (Surkov and Hayakawa 2014).

The response of a resonator to a quasi-steady harmonic driver and an impulsive excitation are quite different. A harmonic driver excites the corresponding eigenmode of the resonator. An impulsive source produces a pulse oscillating between the upper and bottom boundaries of the resonator, gradually spreading because of dispersion. Spectral analysis shows that a sequence of pulses with the time delay $\Delta t$ results in a multi-band spectral structure with the frequency gap $\Delta f=1 / \Delta t$. There is evidence (e.g. Schekotov et al. 2011) that the mechanism of the multi-band spectra formation is not related to the oscillatory response of the IAR, but is caused by the specific impulsive structure of geomagnetic disturbances during the regional lightning activity. In general, it is still uncertain how to discriminate the ULF response to distant thunderstorm centres and regional lightning activity, and what is the relative contribution of those sources to IAR stimulation at mid-latitudes.

Interpretation of basic IAR features requires theoretical models where the refractive in$\operatorname{dex} n_{\mathrm{A}}(z)$ for Alfvén waves in the upper ionosphere is approximated by functions that provide an analytical solution (e.g. Demekhov et al. 2000). The bottom ionosphere was commonly modelled by an impedance layer characterised by the height-integrated Pedersen $\Sigma_{\mathrm{P}}$ and Hall $\Sigma_{\mathrm{H}}$ conductances. When the coupling between modes due to the Hall conductivity (non-diagonal terms of the tensor) is neglected $\left(\sigma_{H} \rightarrow 0\right)$ the system splits into two uncoupled sub-systems for Alfvén and fast magnetoacoustic waves. The mode coupling due to 
Fig. 14 Diurnal variations of the eigenfrequencies (upper panel) and Q-factors (middle panel) for the IAR harmonics $(n=1-4$ indicated near the lines). Bottom panel: The height-integrated Joule dissipation $J=$ $(1 / 2) \int_{0}^{H} \sigma_{\mathrm{P}}\left|e_{1}\right|^{2} d z$ (solid line) and energy flux into the magnetosphere (dashed line)
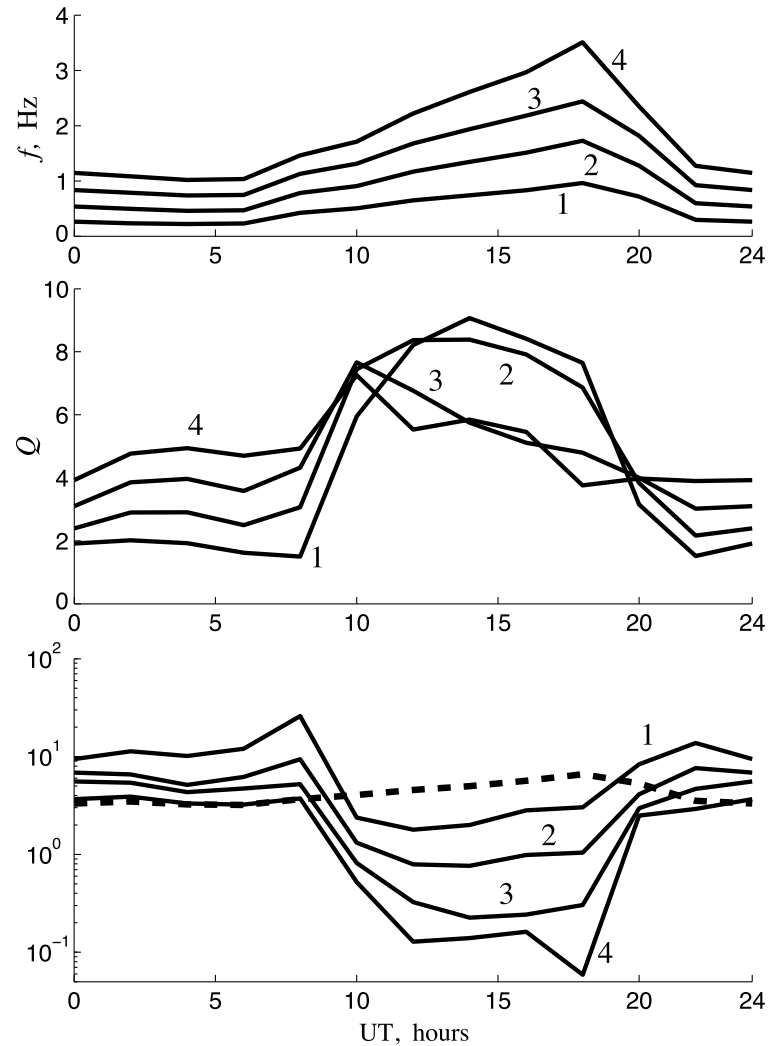

nonzero Hall conductivity may be neglected only if the transverse component of the wave vector is sufficiently large $k_{\perp} \ll\left(\Sigma_{H} / \Sigma_{P}\right)^{2} \mu_{0} \omega \Sigma_{P}$. The boundary problem for this system is to be solved using the measured or modelled profile of dielectric permeability tensor. This solution determines leaky modes and their complex eigenfrequencies.

Profiles of the basic MHD wave parameter along a field line, the Alfvén refractive index $n_{\mathrm{A}}(s)$, are shown for various UT in Fig. 13. Though the decrease rate of the plasma concentration $n_{e}$ with altitude is nearly the same at all UT, the gradient of $n_{\mathrm{A}}(s)$ is steeper during night hours than during daytime hours, due to the contribution of heavy ions into the effective Alfvén velocity. During nighttime a valley is formed in $n_{\mathrm{A}}(s)$ profile between F-layer and E-layer at altitudes about $120-200 \mathrm{~km}$.

The Q-factor is determined by two main energy loss processes: (a) leakage through the upper IAR boundary into the magnetosphere; and (b) Joule dissipation in the conductive layers of the lower ionosphere. The estimate of the height-integrated Joule dissipation $J=(1 / 2) \int_{0}^{H} \sigma_{P}|E|^{2} d z$ shows that for the fundamental mode Joule dissipation is the dominating damping mechanism during daytime, whereas leakage into the magnetosphere becomes most significant during nighttime. For higher harmonics the Joule dissipation rate drops, so wave leakage into the magnetosphere prevails. Diurnal variations of the first four IAR harmonics eigenfrequencies $f_{n}(t)$ and Q-factors $Q_{n}(t)$ are shown in Fig. 14. As expected, IAR eigenfrequencies (upper panel) during the daytime are lower than those for the nightside ionosphere which is due to the higher ionospheric plasma density. The Q-factor (middle panel) is larger during nighttime than during the daytime. The most significant 
contrast is for the lower harmonics $(n=1-2)$. Variations of Q-factor for higher harmonics $(n=3-4)$ are less significant: from about 3-5 during daytime up to about 7 during nighttime.

\section{Coronal Plasma Non-uniformities as Magnetoacoustic Waveguides and Resonators}

As the plasma of the solar corona is observed to be highly filamented along the magnetic field, the main building block of the coronal wave modelling is the theory of MHD modes of a plasma cylinder. This model is used for the description of dynamical processes in a number of plasma structures of the corona, e.g. loops of active regions and post-flare arcades, various filaments, plumes in coronal holes, etc.

Consider a straight cylindrical magnetic flux tube of radius $a$, filled in with a plasma with the properties different from the surrounding plasma. The magnetic field is directed along the axis of the cylinder, that coincides with the $z$-axis. In the simplest case, the equilibrium physical parameters experience a jump at the boundary of the cylinder and are constant elsewhere. Thus, inside the cylinder, the mass density, the plasma temperature and pressure, and the parallel component of the magnetic field are $\rho_{0}, p_{0}, T_{0}$ and $B_{0}$, respectively, while the values of these quantities outside the cylinder are $\rho_{\mathrm{e}}, p_{\mathrm{e}}, T_{\mathrm{e}}$ and $B_{\mathrm{e}}$. The equilibrium condition is the continuity of the total pressure $P_{\text {tot }}$, which is the sum of the plasma and magnetic pressures, across the boundary,

$$
p_{0}+\frac{B_{0}^{2}}{2 \mu}=p_{\mathrm{e}}+\frac{B_{\mathrm{e}}^{2}}{2 \mu} .
$$

The characteristic speeds of MHD waves inside and outside the cylinder are the sound speeds $V_{\mathrm{s} 0}$ and $V_{\mathrm{se}}$, and the Alfvén speeds, $V_{\mathrm{A} 0}$ and $V_{\mathrm{Ae}}$, respectively. It is also convenient to define the so-called tube (or cusp) speeds, $V_{\mathrm{T} 0}$ and $V_{\mathrm{Te}}$, namely $V_{\mathrm{T} 0}=V_{\mathrm{s} 0} V_{\mathrm{A} 0} / \sqrt{V_{\mathrm{s} 0}^{2}+V_{\mathrm{A} 0}^{2}}$. The value of the effective adiabatic index $\gamma_{0}$ that is used in the definition of the sound speed, was recently seismologically estimated in the corona as about 1.1 (Van Doorsselaere et al. 2011).

Linear MHD perturbations of this stable equilibrium are described by the dispersion relation

$$
\rho_{\mathrm{e}}\left(\omega^{2}-k_{z}^{2} V_{\mathrm{Ae}}^{2}\right) \kappa_{0} \frac{I_{m}^{\prime}\left(\kappa_{0} a\right)}{I_{m}\left(\kappa_{0} a\right)}+\rho_{0}\left(k_{z}^{2} V_{\mathrm{A} 0}^{2}-\omega^{2}\right) \kappa_{\mathrm{e}} \frac{K_{m}^{\prime}\left(\kappa_{\mathrm{e}} a\right)}{K_{m}\left(\kappa_{\mathrm{e}} a\right)}=0,
$$

where $\omega$ is the angular frequency, $k_{z}$ is the longitudinal wavenumber, $m$ is the azimuthal wave number, $I_{m}(x)$ and $K_{m}(x)$ are modified Bessel functions of order $m$, the prime denotes the derivative with respect to the argument. The expressions

$$
\kappa_{0}^{2}=-\frac{\left(\omega^{2}-V_{\mathrm{s} 0}^{2} k_{z}^{2}\right)\left(\omega^{2}-V_{\mathrm{A} 0}^{2} k_{z}^{2}\right)}{\left(V_{\mathrm{s} 0}^{2}+V_{\mathrm{A} 0}^{2}\right)\left(\omega^{2}-V_{\mathrm{T} 0}^{2} k_{z}^{2}\right)}, \quad \kappa_{\mathrm{e}}^{2}=-\frac{\left(\omega^{2}-V_{\mathrm{se}}^{2} k_{z}^{2}\right)\left(\omega^{2}-V_{\mathrm{Ae}}^{2} k_{z}^{2}\right)}{\left(V_{\mathrm{se}}^{2}+V_{\mathrm{Ae}}^{2}\right)\left(\omega^{2}-V_{\mathrm{Te}}^{2} k_{z}^{2}\right)} .
$$

are the transverse wave numbers in the internal and external media, respectively. They are equivalent to $k_{r}$ in Eq. (21). Waves that correspond to different solutions to Eq. (24) are referred to as MHD modes of the plasma cylinder. For $\kappa_{\mathrm{e}}^{2}>0$ the perturbations are evanescent outside the cylinder, and are guided along the cylinder. These modes are called trapped (Zaitsev and Stepanov 1982; Edwin and Roberts 1983). Modes with $\kappa_{\mathrm{e}}^{2}<0$ are subject to leakage to the external medium. In this leaky regime the wave energy is guided along the 

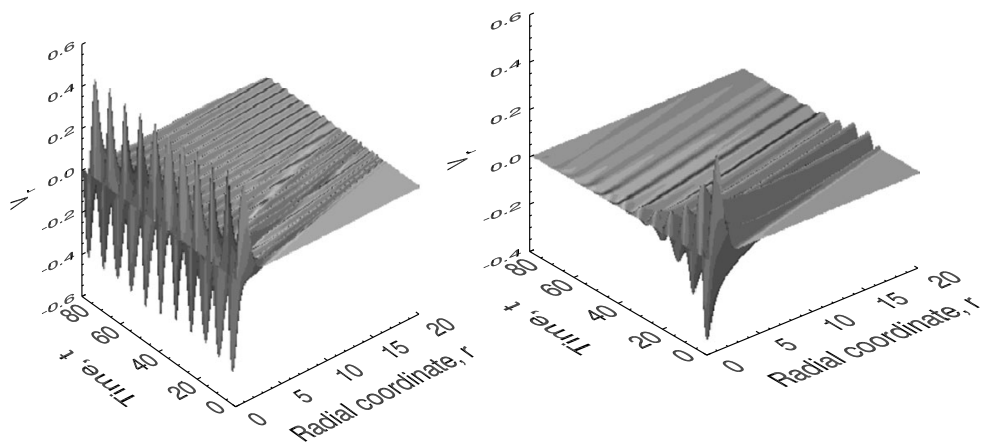

Fig. 15 Variation of the radial velocity in the standing sausage mode of a plasma cylinder, in the trapped (left panel) and leaky (right panel) regimes. From Nakariakov et al. (2012)

Fig. 16 Phase speeds $\omega / k_{z}$ of MHD modes of a field-aligned plasma cylinder as a function of the longitudinal wave number $k_{z}$ for the parameters $V_{\mathrm{A} 0}=2 V_{\mathrm{s} 0}$, $V_{\mathrm{Ae}}=5 V_{\mathrm{s} 0}, V_{\mathrm{se}}=0.5 V_{\mathrm{s} 0}$, $V_{\mathrm{s} 0}=1$. The horizontal dash-dotted lines indicate the characteristic speeds. The solid horizontal line shows the torsional Alfvén waves. The solid curves are solutions for $m=0$, the dotted lines for $m=1$, the dashed lines $m=2$ and the dash-dotted for $m=3$ modes. Figure adapted from Nakariakov and Verwichte (2005)

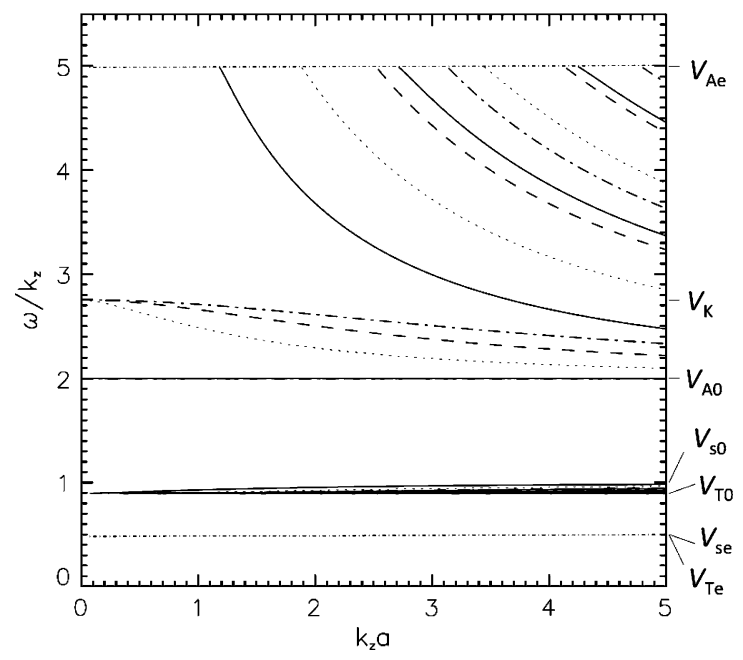

cylinder too, but the waves are continuously radiated to the external medium. Hence, the amplitude inside the cylinder decreases (Cally 1986; Kopylova et al. 2007) that is accounted for by a complex $\omega$. These two regimes are illustrated in Fig. 15 for a standing mode.

Properties of the modes are determined by the physical parameters of the equilibrium. For example, in a typical coronal loop with $V_{\mathrm{A} 0}<V_{\mathrm{Ae}}$ and $V_{\mathrm{se}}<V_{\mathrm{s} 0}$, phase speeds of the trapped modes lie in two bands, the fast band, $\left[V_{\mathrm{A} 0}, V_{\mathrm{Ae}}\right]$, and the slow band $\left[V_{\mathrm{T} 0}, V_{\mathrm{s} 0}\right]$. The general solutions to the dispersion relation (Eq. (24)) are displayed in Fig. 16.

The integer azimuthal wavenumber $m$ determines the modal structure with respect to the angle around the axis of the cylinder (see Fig. 17). In the low- $\beta$ plasma the parameter $m$ is important for the properties of fast modes, and practically does not affect the slow modes. Thus, we consider fast modes of different $m$ only.

Waves with $m=0$ (perturbations are independent on the azimuthal angle with respect to the cylinder axis) are called sausage modes, also known as radial, peristaltic or simply fast magnetoacoustic. A sausage mode is a sequence of axisymmetric expansions and contractions of the cross-section of the cylinder, accompanied by a variation in the plasma density and magnetic field magnitude. Perturbations of the plasma pressure and magnetic field are in phase in this mode. This mode does not perturb the axis of the cylinder. For a fixed wave- 



Fig. 17 Diagrams showing the structure of $m=0$ (sausage) modes (left panel), $m=1$ (kink) modes (middle panel), and $m=2$ (fluting) modes (right panel). The solid line is the equilibrium position of the flux tube, and the dotted and dashed lines two extreme phases

length, a sausage mode has the shortest period of all possible modes in a plasma cylinder (see Fig. 16). A sausage mode has a cut-off value of $\omega$ dependent on the wavelength, that determines the transition from the leaky regime to the trapped regime. In the low- $\beta$ plasma the velocity streamlines for a sausage mode are almost radial.

Waves with $m= \pm 1$ are kink modes that are non-axisymmetric displacements of the cylinder axis with little or no deformation of the loop cross-section. Kink modes with finite wavelengths are compressive, and hence like sausage modes, they have fast magnetoacoustic properties. In the long wavelength limit kink modes become weakly compressive and are sometimes called Alfvénic (Goossens et al. 2012) and related to surface Alfvén waves. In this limit the kink mode phase speed approaches the so-called kink speed

$$
V_{\mathrm{K}}=\left(\frac{\rho_{0} V_{\mathrm{A} 0}^{2}+\rho_{\mathrm{e}} V_{\mathrm{Ae}}^{2}}{\rho_{0}+\rho_{\mathrm{e}}}\right)^{1 / 2} \approx\left(\frac{2}{1+\rho_{\mathrm{e}} / \rho_{0}}\right)^{1 / 2} V_{\mathrm{A} 0},
$$

where the second expression corresponds to the low- $\beta$ plasma. In this regime a kink mode is characterised by periodic radial and azimuthal velocity streamlines. Waves with higher $m$ are referred to as flute or ballooning modes. They have not been convincingly identified in coronal observations, and hence they are not discussed in this review further.

Longitudinal (or slow) modes are constituted by mainly field-aligned compressive motions. In the short wavelength limit these modes reduce to usual acoustic waves and propagate at the sound speed $V_{\mathrm{s} 0}$. Perturbation of the plasma pressure and magnetic field are in anti-phase in this mode. In the long-wavelength limit, longitudinal modes propagate at the tube speed $V_{\mathrm{T} 0}$. In this regime the local wave vector is almost perpendicular to the field, while the bulk flows induced by this mode remain mainly field-aligned. As longitudinal modes with different azimuthal wavenumbers $m$ have in a low- $\beta$ plasma very close dispersion curves, usually, possibly erroneously, they are considered as a single mode. However, higher $|m|>0$ slow modes should result in oppositely directed parallel velocities in the loop.

In cylinders with smooth radial profiles of the Alfvén speed individual surfaces of constant Alfvén speed support propagation of incompressive Alfvén waves called torsional waves. In these waves the plasma moves in the azimuthal direction only, and hence they can be considered as waves of alternate vorticity and electric current density. Torsional waves do not perturb the cylinder boundary radially, i.e. compressively. In the solar context, torsional waves are not considered as modes, as they are not collective: their spectrum is continuous 
as different surfaces of constant Alfvén speed support waves with different periods and/or wavelengths, that do not interact with each other in an ideal plasma. In contrast, sausage, kink, flute and longitudinal modes are collective, disturbing the whole cylinder. Moreover, in contrast with MHD modes that are subject to geometrical dispersion, torsional waves are dispersionless.

A dispersion relation analogous to Eq. (24) can be written for other plasma configurations, e.g. for a field-aligned slab or a current sheet. Properties of MHD modes of a slab are mainly similar to cylinder modes (see, e.g. Edwin and Roberts 1982). The major difference is that slab kink modes tend to the external Alfvén speed in the long wavelength limit, instead of the kink speed. In a number of theoretical models, the slab geometry is used instead of the cylindrical geometry, because of the analytical simplicity.

Despite its obvious simplicity, the theory of MHD modes of a straight plasma cylinder with a sharp boundary, provides a solid and commonly accepted basis for the study of MHD oscillations and waves in various plasma configurations of the solar corona. The generalisation of this theory on the important case of the smooth boundary is discussed in Sect. 5.5. Another potentially important effect, of the loop curvature, was shown to be not significant for MHD mode periods (Van Doorsselaere et al. 2004b).

\section{MHD Mode Conversion}

A linear resonant conversion of the fast mode into the Alfvén mode can occur on some distinct magnetic (resonant) shells. In the magnetospheric and coronal physics, this phenomenon plays rather different roles: while in the former it is considered as a generation mechanism for the observed (usually, Alfvén) modes or a way to interpret its observed spatial structure, in the latter it is considered as a mechanism for the absorption of the energy of observed collective modes, i.e. their linear transformation in unresolved smallscale Alfvénic motions, and associated plasma heating. However, the physics behind this phenomenon is similar in both branches of MHD wave physics.

\subsection{The Box Model}

The simplest model which allows one to take into account the plasma inhomogeneity is the box model, where the field lines are considered to be straight and the Alfvén speed depends only on the perpendicular (e.g. corresponding to the radial direction in the magnetospheric geometry or in coronal flux tubes) coordinate $x$, across the field directed in the $z$-direction (see Fig. 18). In this model all perturbed values can be chosen to depend on the e.g. azimuthal $(y)$ and parallel $(z)$ coordinates as $\propto \exp \left(-i \omega t+i k_{y} y+i k_{\|} z\right)$.

The linearised MHD equations describing the structure of coupled Alfvén and fast magnetoacoustic modes in the box model can be combined into a single ordinary differential equation for the wave component $b_{z}$

$$
b_{z}^{\prime \prime}-2 \frac{\kappa_{\mathrm{A}}^{\prime}}{\kappa_{\mathrm{A}}} b_{z}^{\prime}+\kappa_{\mathrm{F}}^{2} b_{z}=0,
$$

where the prime means differentiation with respect to the radial coordinate (e.g. $\kappa_{\mathrm{A}}^{\prime}=$ $\left.d \kappa_{\mathrm{A}} / d x\right)$, and

$$
\kappa_{\mathrm{A}}^{2}=k_{\mathrm{A}}^{2}-k_{\|}^{2}, \quad \kappa_{\mathrm{F}}^{2}=k_{\mathrm{A}}^{2}-k_{\|}^{2}-k_{y}^{2},
$$


Fig. 18 Schematic illustration of the correspondence between the magnetospheric box model and realistic magnetosphere. From Glassmeier et al. (1999)

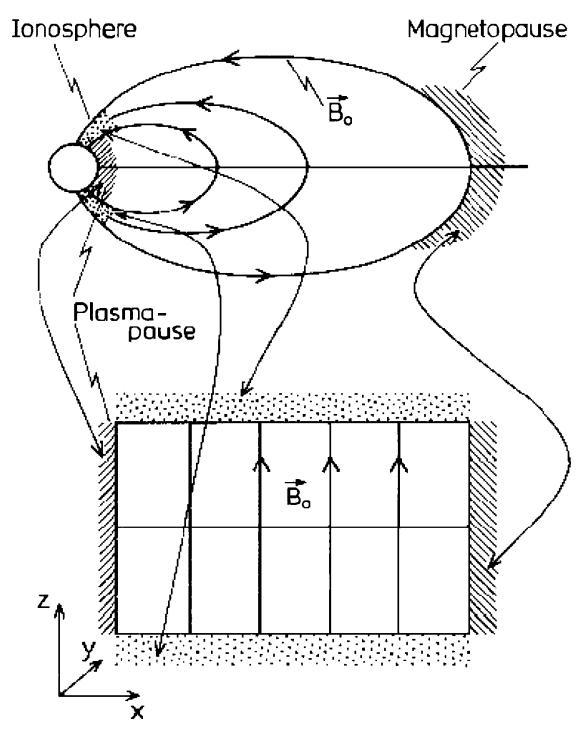

where $k_{\mathrm{A}}=\omega / V_{\mathrm{A}}$. For such a 1D inhomogeneity the mathematical structure of the MHD equations is similar (but not identical) to Maxwell's equations for resonant conversion of electromagnetic waves into plasma oscillations.

Equation (27) has two characteristic points:

- the singularity point $x_{\mathrm{A}}$ at $\omega=k_{\|} V_{\mathrm{A}}(x)$, where $\kappa_{\mathrm{A}}=0$;

- the reflection point $x_{\mathrm{F}}$ at $\omega^{2}=\left(k_{y}^{2}+k_{\|}^{2}\right) V_{\mathrm{A}}^{2}(x)$, where $\kappa_{\mathrm{F}}=0$ and the effective wave number of WKB solution $k_{x}=k_{\mathrm{F}}$ tends to zero.

Since the resonant point is situated on the magnetic surface where the Alfvénic dispersion relation $\omega=k_{\|} V_{\mathrm{A}}$ holds, it corresponds to resonant Alfvén mode excitation on the resonant surface (the field line resonance in the magnetospheric physics). The Alfvén mode is subject to effective dissipation by small but finite viscosity or resistivity, because of very steep gradients in the radial direction. Thus, the singularity at $x_{\mathrm{A}}$ results in the energy transfer towards the resonant surface, its accumulation in its vicinity, absorption of the wave energy and subsequent plasma heating on the resonant surface.

The reflection point $x_{\mathrm{F}}$ is a signature of the fact that the fast mode propagating from regions of the low Alfvén speed cannot reach the resonant point being reflected back. However, a part of the fast mode energy is tunnelling deeper into the plasma (because of the exponentially evanescent solution). It is that tunnelled energy that generates the Alfvén mode on the resonant magnetic shell. This phenomenon is usually called the Alfvén resonance.

The Alfvén resonance and transparency regions for the fast magnetoacoustic mode are spatially separated from one another, so the mode conversion has the character of sub-barrier tunnelling. The distance between the resonant and reflecting shells depends on the azimuthal wave vector value $k_{y}$. If $k_{y}$ is large (azimuthally small-scale waves) compared with the inverse inhomogeneity scale in plasma, then only the exponentially small part of the wave energy can penetrate deep into the magnetosphere. In this case, the resonant mechanism of the Alfvén mode generation is ineffective and one must search for the wave's sources in situ. This is why the magnetospheric physicists believe that the Alfvén waves with high azimuthal wave numbers $m$ are generated by some internal processes (see Sect. 9.2 for more detail). 


\subsection{MHD Wave Conversion in the Terrestrial Magnetosphere}

Alfvén waves are very distinct from common wave processes in optics, acoustics, or geoseismology. Their spectrum is continuous even in a bounded system, and the eigenfunctions are singular (see also the discussion of torsional Alfvén waves in Sect. 4). These features result in interesting peculiarities of for Alfvén waves: one-dimensional propagation patterns and absence of geometrical attenuation, the possibility of transporting non-steady field-aligned currents to large distances along field lines, the absence of steady oscillations in non-dissipative systems, and the irreversible conversion of fast magnetoacoustic disturbances into Alfvén waves (see discussion in Sect. 5.1).

According to magnetospheric resonant theory, when MHD disturbances from the outer magnetosphere become incident upon it they are transformed into field line Alfvén oscillations. This mechanism for resonant conversion of fast magnetoacoustic waves into Alfvén waves is used to interpret the occurrence of narrow-band ULF signals in the Earth's magnetosphere.

The basic notions about magnetospheric Alfvén resonators were originally formulated in the frameworks of simplified 1D models (see Sect. 5.1). For a more general, 3D geometry, when the variables are not separable, the asymptotic singular solution in the vicinity of the resonant shell can be obtained by implementing the Frobenius method (e.g. Goossens et al. 1985; Kivelson and Southwood 1986):

$$
\begin{aligned}
b_{z} & \sim\left[k_{y}\left(x-x_{\mathrm{A}}\right)\right]^{2} \ln \left[k_{y}\left(x-x_{\mathrm{A}}\right)\right], \\
b_{x}, e_{y} & \sim \ln \left[k_{y}\left(x-x_{\mathrm{A}}\right)\right], \\
b_{y}, e_{x} & \sim\left[k_{y}\left(x-x_{\mathrm{A}}\right)\right]^{-1} .
\end{aligned}
$$

Here $x$ is the coordinate along the meridian; $x_{\mathrm{A}}(f)$ is the coordinate of the resonant magnetic shell that corresponds to the frequency $f$. The singular growth of an electromagnetic field amplitude at a resonant shell, where $x=x_{\mathrm{A}}(f)$, is terminated by dissipative effects, e.g., Joule damping in the conductive ionospheres. To account for this effect, the term $x-x_{\mathrm{A}}(f)$ must be replaced with $x-x_{\mathrm{A}}(f)+i \delta$, where the semi-width of the resonant spatial peak $\delta$ is produced by a dissipation. Thus, a qualitative pattern of MHD wave conversion and singular wave structure in the vicinity of Alfvén resonance remain valid even in multi-dimensional inhomogeneous systems. The $b_{x}$ component has a weaker logarithmic singularity near the resonance in the magnetosphere, so the resonant behaviour of this component would hardly be noticeable.

The spatial structure of ULF waves can be qualitatively imagined as a superposition of a source field and the resonant response to it from the magnetospheric Alfvén resonator. This resonator is formed by geomagnetic quasi-dipole field lines terminated by the conjugate ionospheres. The source field is due to the large-scale fast magnetoacoustic mode. The resonant response of the magnetosphere, caused by Alfvén field line excitation, is strongly localised and produces a steep enhancement of amplitude and strong phase gradient upon the transition across the resonant shell. This transformation is most effective at the geomagnetic latitude $\Phi$, where the source frequency $f$ matches the local frequency $f_{\mathrm{A}}$ of field line Alfvén oscillations, namely $f \simeq f_{\mathrm{A}}(\Phi)$ (Southwood 1974; Chen and Hasegawa 1974). Upon the transmission of ULF waves through the ionosphere their essential spatial structure mainly survives, but the polarisation plane rotates by $\pi / 2$ and some broadening of the resonant peak occurs. 
MHD disturbances from remote parts of the magnetosphere propagate inside the magnetosphere and, through mode transformation, they excite standing Alfvén oscillations on the Earth's magnetic field lines. Alfvén waves incident on the ionosphere are, in most cases, the sources of ULF geomagnetic pulsations (Pc3-5) observed on the ground. The leading term in the expansion, which describes the resonant singularity of $b_{y}(x, f)$ near the resonant shell, takes the form

$$
b_{y}(x, f)=b_{0}(f) \frac{i \delta_{i}}{x^{\prime}+i \delta_{i}},
$$

where $x^{\prime}=x-x_{\mathrm{A}}(f)$ is the distance from the resonant shell, $x$ is the coordinate of a magnetic shell, $x_{\mathrm{A}}(f)$ is the point where $f=f_{\mathrm{A}}(x), \delta_{i}$ is the full-width half-maximum of the resonance region above the ionosphere, and $b_{0}$ is the amplitude of the perturbation. The resonance width $\delta_{i}$ is related to the damping rate $\gamma$ by the relationship $\delta_{i}=-\gamma\left(2 \pi \partial f_{\mathrm{A}} / \partial x\right)^{-1}$ (whereas $\gamma>0$ ). The gradient sign of the Alfvén frequency, and correspondingly $\delta_{i}$, determines the direction of an apparent phase velocity in the radial direction of the ULF waves in the resonant region. Throughout the magnetosphere (except at the plasmapause) $\partial f_{\mathrm{A}} / \partial x<0$ and the meridional component of the phase velocity is directed towards higher latitudes.

Pumping of wave energy into the resonant region causes both growth and narrowing of the spatial resonant peak. In the steady state this growth is saturated at some level depending on the rate of the dominant dissipation mechanism. Commonly, Joule dissipation is taken to be a damping mechanism in the ionosphere. The damping rate $\gamma_{n}$ of the $n$-th mode in the magnetospheric resonator is related to the width of the resonant region $\delta_{n}$ by the simple relationship

$$
\left|\gamma_{n} / \omega_{\mathrm{A} n}(x)\right|=\left|\delta_{n} / a\right|=\frac{1}{\pi n} \ln |R|,
$$

where $a=\left|\omega_{\mathrm{A} n}(x)\left(d \omega_{\mathrm{A} n}(x) / d x\right)^{-1}\right|$ is the typical scale of Alfvén frequency spatial variations, and $R$ is the Alfvén wave reflection coefficient from the ionosphere.

In general, the magnetosphere behaves as a giant maser for MHD waves. The solar wind flow pumps energy into this maser, then the seed disturbances are band-filtered in the magnetospheric Alfvén resonator, and are transmitted through the semi-transparent windows (the ionosphere). Besides the solar wind input, the injection of non-equilibrium energetic particles into the magnetosphere, also can generate monochromatic waves in such a maser, similar to the inverse population in lasers. The maser mechanism results in the appearance in a space plasma of various narrow-band quasi-monochromatic oscillations, despite the turbulent character of the solar wind-magnetosphere interaction. In a greater detail, the excitation of the magnetospheric Alfvén resonator is considered in Sect. 9.

Despite a significant progress in our understanding of MHD wave coupling in the magnetosphere, there still remain important open questions:

- Though there have been a few reports of the signatures of the field-line resonant conversion recorded by spaceborne magnetometers, the evidence of the ULF wave resonant conversion in situ is almost absent. Is it just because we had not have enough time to find them, or there are some deep physical reasons for that?

- More realistic theory of the resonant conversion, taking into account the 3-D inhomogeneity of the magnetosphere and time-limited character of the external driver must be developed and compared with observations. 
Fig. 19 Schematic illustration of the model of a plasma with a longitudinally non-uniform density $\rho(z)$. The arrows show the wave vectors of fast magnetoacoustic (M) and Alfvén (A) waves

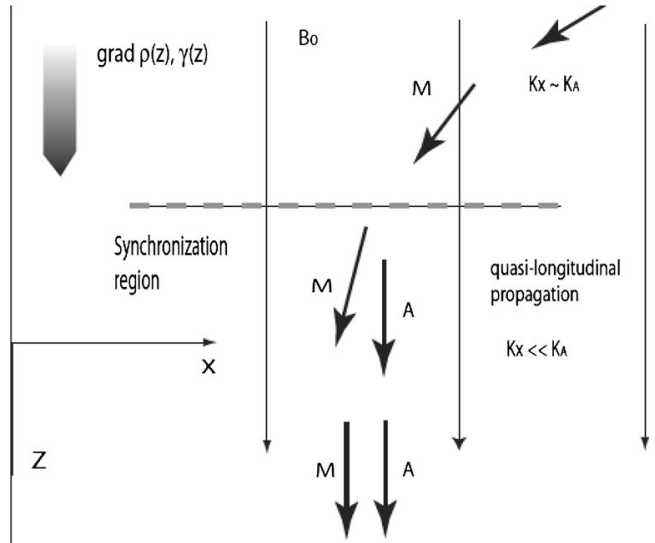

\subsection{MHD Wave Coupling on Open Field Lines in the Magnetosphere}

In the standard Alfvén resonance model, Alfvén field line oscillations are considered to be generated in a bounded, inhomogeneous across the equilibrium magnetic field plasma due to resonant conversion of fast magnetoacoustic perturbations. Alfvén modes are excited in the form of waves standing along closed magnetic field lines bounded by their reflecting ends (the conductive ionospheres). Though resonance theory has turned out to be very successful in the interpretation of amplitude-phase ULF wave distributions along latitude, the occurrence of quasi-monochromatic waves in an unbounded plasma is a challenge for space physics theorists, because the standard model cannot be directly applied to such systems.

An alternative model was suggested by Pilipenko et al. (2008), where conversion of propagating compressive fast magnetoacoustic modes into propagating Alfvén waves occurs on open field lines owing to the finite frequency effect, $\delta_{\omega}=\omega / \Omega_{\mathrm{i}} \ll 1$, where $\Omega_{\mathrm{i}}$ is the ion gyrofrequency (see Fig. 19). They considered the model of a cold plasma immersed in a straight uniform magnetic field $\mathbf{B}_{0}$. The Alfvén velocity along $\mathbf{B}_{0}$ is non-uniform, $V_{\mathrm{A}}(z)$, because of the non-uniformity of the plasma density profile $\rho(z)$. As a fast magnetoacoustic wave with the transverse wave vector component $k_{x}=k_{\perp}$ propagates in such an inhomogeneous plasma, its wave vector rotates due to refraction and, accordingly, the longitudinal wave vector component $k_{\|}^{(\mathrm{M})}(z)$, where the superscript (M) denotes the fast wave, changes. The plasma dielectric tensor components $\varepsilon$ and $g$ are described by the approximate expressions

$$
\varepsilon=1+\frac{\omega_{\mathrm{pi}}^{2}}{\Omega_{\mathrm{i}}^{2}-\omega^{2}} \simeq \frac{c^{2}}{V_{\mathrm{A}}^{2}}, \quad g=\frac{\omega \omega_{\mathrm{pi}}^{2}}{\Omega_{\mathrm{i}}\left(\Omega_{\mathrm{i}}^{2}-\omega^{2}\right)} \simeq \delta_{\omega} \frac{c^{2}}{V_{\mathrm{A}}^{2}} .
$$

The finite-frequency effects $\propto \delta_{\omega}$ are taken into account because they govern the interaction between the fast magnetoacoustic and Alfvén modes.

ULF waves on open or extended field lines may be considered in the WKB approximation, valid up to $(k L)^{-1}<0.5$, where $L$ is the scale of inhomogeneity. In this regime the coupled equations for the amplitudes of Alfvén and fast magnetoacoustic waves, $a_{\mathrm{A}}$ and $a_{\mathrm{M}}$, propagating along the magnetic field, are as follows

$$
\begin{aligned}
& d a_{\mathrm{A}} / d z=i k_{\mathrm{A}} a_{\mathrm{A}}-C a_{\mathrm{M}}, \\
& d a_{\mathrm{M}} / d z=i k_{\mathrm{M}} a_{\mathrm{M}}+C a_{\mathrm{A}} .
\end{aligned}
$$


Fig. 20 Frequency dependence of the conversion coefficient $\alpha(\Omega)$, numerically calculated for a modelled profile $\rho(z)$, for different values of normalised wave vector $K$

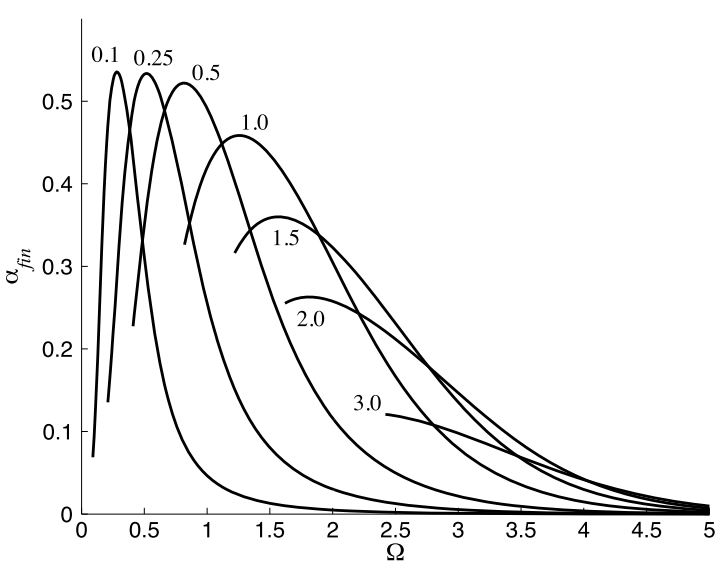

In the MHD limit, the field-aligned wave numbers of Alfvén and fast magnetoacoustic waves are described by the expressions

$$
\left[k_{\|}^{(\mathrm{A})}\right]^{2}=k_{\mathrm{A}}^{2}-\frac{k_{x}^{2}}{2}\left(1-\sqrt{1+\gamma_{\mathrm{d}}^{2}}\right), \quad\left[k_{\|}^{(\mathrm{M})}\right]^{2}=k_{\mathrm{A}}^{2}-\frac{k_{x}^{2}}{2}\left(1+\sqrt{1+\gamma_{\mathrm{d}}^{2}}\right),
$$

where the factor $\gamma_{\mathrm{d}}=2 \delta_{\omega}\left(\omega / k_{x} V_{\mathrm{A}}\right)^{2}$. The mode coupling coefficient in equations (33)

$$
C=\frac{k_{\|}^{(\mathrm{A})}+k_{\|}^{(\mathrm{M})}}{4 \sqrt{k_{\|}^{(\mathrm{A})} k_{\|}^{(M)}}} \frac{d \gamma_{\mathrm{d}} / d z}{1+\gamma_{\mathrm{d}}^{2}}
$$

is determined by the longitudinal variation of the factor $d \gamma_{\mathrm{d}} / d z \propto d \rho / d z$.

In a longitudinally inhomogeneous plasma with sufficiently small $\delta_{\omega}$ there exists a region where $k_{\|}^{(\mathrm{A})}$ and $k_{\|}^{(\mathrm{M})}$ approach one another. Analysis of the set of equations (33) has shown that at the frequency, when the mismatch between the wave numbers is low and the coupling coefficient $C$ is high, an efficient mode conversion occurs. In this case the waves are synchronised: their longitudinal wave numbers are close to one another, and the phase difference between the fast magnetoacoustic and Alfvén modes, $\approx \pi$, is stable.

It is convenient to introduce the normalised frequency and transverse wavenumber, $\Omega$ and $K_{\text {trans }}$, through the relationships $\Omega=\delta_{\omega}\left(L / \lambda_{\mathrm{i}}\right)^{1 / 2}$ and $K_{\text {trans }}=k_{x} L\left(L / \lambda_{\mathrm{i}}\right)^{-1 / 4}$, where $\lambda_{\mathrm{i}}=V_{\mathrm{A} 0} / \Omega_{\mathrm{i}}$ is the ion inertia length. The frequency dependence of the conversion coefficient $\alpha(\Omega)=\frac{\left|a_{\mathrm{A}}\right|}{\sqrt{\left|a_{\mathrm{A}}\right|^{2}+\left|a_{\mathrm{M}}\right|^{2}}}$, numerically calculated for a modelled profile $\rho(z)$ and for different values of $K$, has a maximum at $\Omega \simeq 0.4-1.6$ (Fig. 20). Near the maximum, the conversion is fairly efficient (the conversion coefficient reaches $50 \%$ ).

Thus, in the region where a fast magnetoacoustic wave propagates in the quasilongitudinal regime, it is partially converted into an Alfvén wave. The conversion is most efficient in the synchronisation region, where the longitudinal components of the wave vectors approach one another and the wave phases are synchronised. Upon further propagation, the two waves escape from the synchronisation region and continue to propagate independently of one another. The excitation efficiency of an Alfvén wave depends on frequency, and is the highest at the dimensionless frequencies $\Omega \simeq K_{\text {trans }}^{2 / 3}$. This conversion mechanism ensures selective amplification of Alfvén waves at certain frequencies, resulting in the resonant conversion of running fast magnetoacoustic waves into Alfvén waves in an unbounded 
plasma. This conversion mechanism can be applied to interpret the occurrence of quasimonochromatic wave trains in the magnetospheres of giant planets (Cramm et al. 1998) or on open field lines in the solar corona.

\subsection{Ground Monitoring of the Magnetospheric Plasma Density with the Use of ULF Waves}

MHD mode conversion opens up a number of interesting perspectives for diagnostics of the magnetospheric plasma. There is a principal distinction between monitoring the Earth's interior with seismic waves and the magnetospheric plasma with ULF waves. In contrast to geoseismology, the properties of ULF wave sources (location, spectral content, etc.) are known only approximately. Nonetheless, we can still use ground based observations of ULF waves for MHD diagnostics ("seismology of the magnetosphere" or "magnetoseismology"). The physical basis of this method of plasma diagnostics is quantifying the effect of resonant conversion of MHD waves in the inhomogeneous magnetosphere. The resonant frequency $f_{\mathrm{A}}(\Phi)$ for a given field line is determined by the local field-aligned distribution of plasma, whereas a quality factor $Q$ is determined by the dissipative properties of the ionosphere and magnetosphere. In particular, reliable identification of the resonant frequency opens up the possibility of monitoring the magnetospheric plasma density via ground-based data.

The principal problem of determining the magnetospheric Alfvén resonator parameters is linked to the fact that in most events the contribution to the ULF spectral content of the resonant magnetospheric response and of the one from the "source" are comparable. So in most cases, a spectral peak does not necessarily correspond to a local resonant frequency, and the width of a spectral peak cannot be directly used to determine the quality factor of the magnetospheric resonator.

The most effective way the influence of the source spectrum can be discarded and local resonant effects can be retrieved is with the use of gradient methods, based on measurements of ULF field at two latitudinally separated stations with a small base. The resonant features can be also highlighted by polarisation methods, based on asymmetry between the resonant response of various ULF components at the observational site. Another method, travel time seismology of the magnetospheric plasma, makes use of the propagation properties of different magnetospheric modes.

The final step in each of the techniques is inversion of the observed properties (resonant frequencies, arrival times) to obtain plasma mass densities. It requires the solution of the governing wave equation along the field line. This step requires some description of the topology and strength of the magnetic field. At mid latitudes during quiet conditions the dipole approximation of the magnetic field can be sufficient, however at lower and higher latitudes a more realistic representation of the field is needed. Field lines starting from low latitudes are so close to the Earth that higher order harmonics of the terrestrial main field has to be taken into account, e.g. by using some terrestrial geomagnetic field model, while at higher latitudes the contribution of external magnetospheric sources needs to be included by using some empirical magnetospheric model. Ignoring the deviation of the magnetic field topology from the dipole case may lead to under- or overestimation of the mass density by at least $30 \%$ at low latitudes under quiet conditions, whereas at high latitudes under disturbed conditions the error can probably exceed $100 \%$.

In some circumstances, if multiple harmonics are observed, the plasma mass density at off-equator points can also be estimated. Price et al. (1999) developed the harmonic derived density method for estimating the plasma mass density without any a priori assumption on the functional form of the density distribution. However, the applicability of the method is 
highly constrained by the insensitivity of the resonant frequency of the supposed distribution function. Information on plasma mass densities beyond $\sim 20^{\circ}$ from the equatorial plane is unavailable, at least, using the upper harmonics.

Some assumption on the functional form of the plasma mass density distribution along field lines is also necessary. Typically, the field aligned distribution is approximated by a power law distribution, which is sufficient in most cases, except for the lowest latitudes, where contribution of heavier ionospheric ions makes the distribution more complicated. The resonant frequency is determined by the section of the field line, where the wave spends most of the time, i.e. where the Alfvén speed has a minimum: near the equator. Hence, the inversion is rather insensitive to the choice of the plasma distribution away from the magnetospheric equator.

\subsubsection{Gradient Method of the Magnetospheric Plasma Diagnostics}

The amplitude and phase characteristics of the north magnetic field component $H$ at the ground can be modelled by the function

$$
H(x, f)=\frac{h_{\mathrm{A}}(f)}{1-i \zeta}
$$

where $\zeta=\left(x-x_{\mathrm{A}}\right) / \delta$ denotes the normalised distance from the resonant point $x_{\mathrm{A}}(f), h_{\mathrm{A}}(f)$ is the amplitude of the pulsation at the resonant point, and $x$ is the coordinate of the magnetic shell, as measured along the geomagnetic meridian. Upon transmission through the ionosphere, the width $\delta$ of the resonance peak, as observed at the ground, is increased compared with that above the ionosphere, $\delta_{\mathrm{i}}$, namely $\delta=\delta_{\mathrm{i}}+h$, where $h$ is the height of the ionospheric E-layer.

Despite the apparent simplicity of the resonance model, the theoretically predicted amplitude and phase meridional structure given by Eq. (35) corresponds well to the observed local structure of ULF waves. Precise measurements of the gradients of the spectral amplitude and phase along a small baseline allow one to exclude the influence of the source spectrum form and to reveal even relatively weak resonant effects. The following simple relationships, stemming from the properties of the function (35), describe the specific features of the ratio $G(f)$ between the amplitude spectra and the difference of phase spectra $\Delta \psi(f)$ of the north components of the magnetic field $H$, recorded at points $x_{1}$ and $x_{2}$ $\left(\Delta x=x_{1}-x_{2}>0\right)$ :

$$
G(f)=\frac{\left|H\left(x_{1}, f\right)\right|}{\left|H\left(x_{2}, f\right)\right|}=\left[\frac{1+\zeta_{2}^{2}}{1+\zeta_{1}^{2}}\right]^{1 / 2}, \quad \Delta \psi(f)=\arctan \left(\frac{\zeta_{2}-\zeta_{1}}{1+\zeta_{1} \zeta_{2}}\right)
$$

The typical features of functions (36) are as follows:

(a) $G\left(f_{\mathrm{A}}\right)=1$ for the frequency $f=f_{\mathrm{A}}\left(x_{c}\right)$, where the point $x_{c}=\left(x_{1}+x_{2}\right) / 2$ is located at the midpoint between the stations;

(b) $G(f)$ reaches the extreme values $G_{+}$and $G_{-}$at the frequencies $f_{+}$and $f_{-}$which correspond to the points $x_{ \pm}=x_{\mathrm{c}} \pm\left[\delta^{2}+(\Delta x / 2)^{2}\right]^{1 / 2}$

(c) $G_{+} \times G_{-}=1$ and $G_{+}-G_{-}=\Delta x / \delta$;

(d) $\Delta \psi(f)$ reaches an extreme value $\Delta \psi^{*}=2 \arctan (\Delta x / 2 \delta)$ at the frequency $f_{\mathrm{A}}$.

The properties (a) and (d) of the functions $G(f)$ and $\Delta \psi(f)$ enable one to estimate the resonant frequency $\left(f_{\mathrm{A}}\right)$ of the field line between the stations (i.e. at $x_{\mathrm{c}}$ ) as the key parameter 
Fig. 21 The dynamic gradient method technique: the spectral transfer function $G(f)$ (upper panel) and phase delay $\Delta \psi(f)$ (bottom panel)

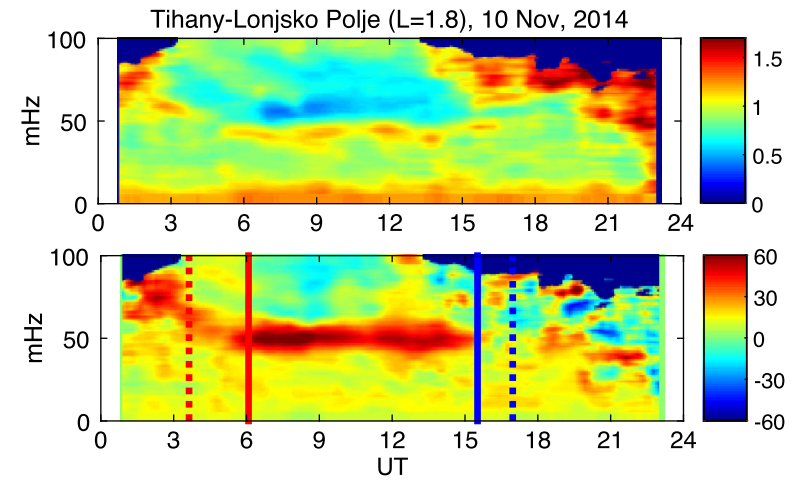

for plasma diagnostics. The width of the resonant peak can be directly reconstructed from the extreme values of the phase difference as

$$
\delta=\frac{\Delta x}{2} \operatorname{cotan}\left(\frac{\Delta \psi^{*}}{2}\right) .
$$

An example of results of the gradient method applied to stations from EMMA magnetometer array is shown in Fig. 21. Characteristic features, predicted by the resonant theory, are evident: the transfer of the amplitude ratio (transfer function) across 1 , and the extreme value of the phase delay at the resonant frequency $f_{\mathrm{A}}$.

The gradient method with some modification can also be applied to the observations at lateral geoelectrically inhomogeneous Earth's crust. The standard gradient method uses only the amplitude ratio or phase difference between signals for the determination of the resonant frequency of the field line mid-way between the observational stations. The advanced amplitude-phase gradient method (APGM) which employs both amplitude and phase information at the same time allows not only to determine the resonant frequencies of some particular field lines, but also to restore their latitudinal profile. The continuous $f_{\mathrm{A}}(x)$ distribution can be obtained from the dependence of the resonance position $x_{\mathrm{A}}(f)$ on frequency, as

$$
\frac{x_{\mathrm{A}}(f)-x_{1}}{\Delta x}=\frac{1-G \cos (\Delta \psi)}{G^{2}-2 G \cos (\Delta \psi)+1} .
$$

Then, inverting the dependence $x_{\mathrm{A}}(f)$, the radial profile $f_{\mathrm{A}}(x)$ can be restored.

Another method, suggested to restore key parameters of the resonant structure and continuous latitudinal distribution of resonant frequency, is based on the fractional-linear transform of the gradient data into the complex plane, whereas the resulting image is the hodograph. The hodograph method is a powerful and convenient tool for data analysis. The availability of several mutually controlling methods enables one to perform a reliable monitoring of the resonant frequency variations.

\subsubsection{Travel Time Magnetoseismology}

This plasma diagnostics technique is based on the detection of the arrival times of Alfvén waves driven by the same source. The idea is based on the notion of MHD wave transmission in the magnetosphere as propagation of isolated wave packets. During the propagation through the non-uniform magnetosphere, a conversion of fast magnetoacoustic waves into 
Fig. 22 Schematic illustration of the MHD wave packet propagation in the magnetosphere. Upon such propagation, a conversion of fast magnetoacoustic into Alfvén wave packets occurs

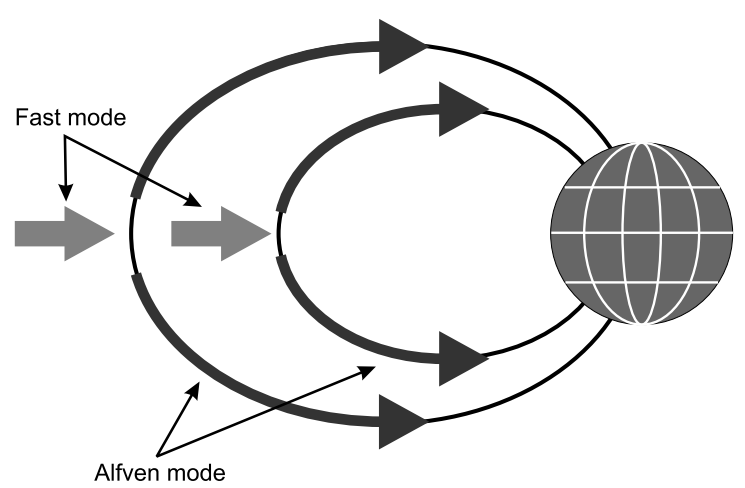

Alfvén waves occurs, as illustrated in Fig. 22. The travel time technique assumes a spatially localised impulsive source (such as the initial pulse of a geomagnetic storm at the nose of the magnetopause or substorm onset in the magnetotail) which launches fast magnetoacoustic waves that can propagate isotropically in the magnetosphere at the radially varying Alfvén speed. The fast waves propagating across magnetic field lines couple to Alfvén modes, which propagate further along the curved field lines. The waves arrive at the ionosphere at different times along different field lines (e.g. the travel time from the apex of the field line to its footpoint is the quarter of the Alfvénic eigenperiod of the considered field line). The inversion starts from the arrival time differences between different spatial locations of the observations, and infers the equatorial plasma mass density for the field lines the observations are available for.

Realising that real processes are much more complicated than the above described ideal case, several attempts have been made to improve the travel time technique, by taking into account wave refraction, ionospheric effects, etc., however, further corrections are foreseen (Menk and Waters 2013).

It is worth noting the physical similarity of the model for travel time seismology (Fig. 22) and the possible interpretation of the global coronal wave (Fig. 6). However, the measured wave signal is in both cases different: for the global coronal wave, it is a fast magnetoacoustic wave, while for travel time seismology, the arrival time of Alfvén waves is measured. Still, the development of travel time seismology in the solar corona may yield interesting new results.

\subsection{Effect of Resonant Conversion/Absorption in Coronal Loops}

Modelling of MHD oscillations of coronal plasma structures in terms of the modes of a magnetic flux tube with a sharp boundary with a discontinuity in density, magnetic field and temperature, discussed in Sect. 4, misses an important effect of coupling of the collective modes with local Alfvénic oscillations of individual magnetic surfaces. In the solar atmosphere, the magnetic flux tubes are likely to have a continuous transition from their core density and magnetic field to the parameters in the surrounding plasma. Such a continuous transition introduces new physics. For simplicity, let us assume a homogeneous magnetic field and a density profile in a cylindrical flux tube of radius $a$ as follows

$$
\rho(r)= \begin{cases}\rho_{\mathrm{e}} & \text { for } r>a+l / 2, \\ \rho_{\mathrm{e}}+\left(\rho_{0}-\rho_{\mathrm{e}}\right) f(r) & \text { for } a-l / 2 \leq r \leq a+l / 2, \\ \rho_{0} & \text { for } r<a-l / 2,\end{cases}
$$



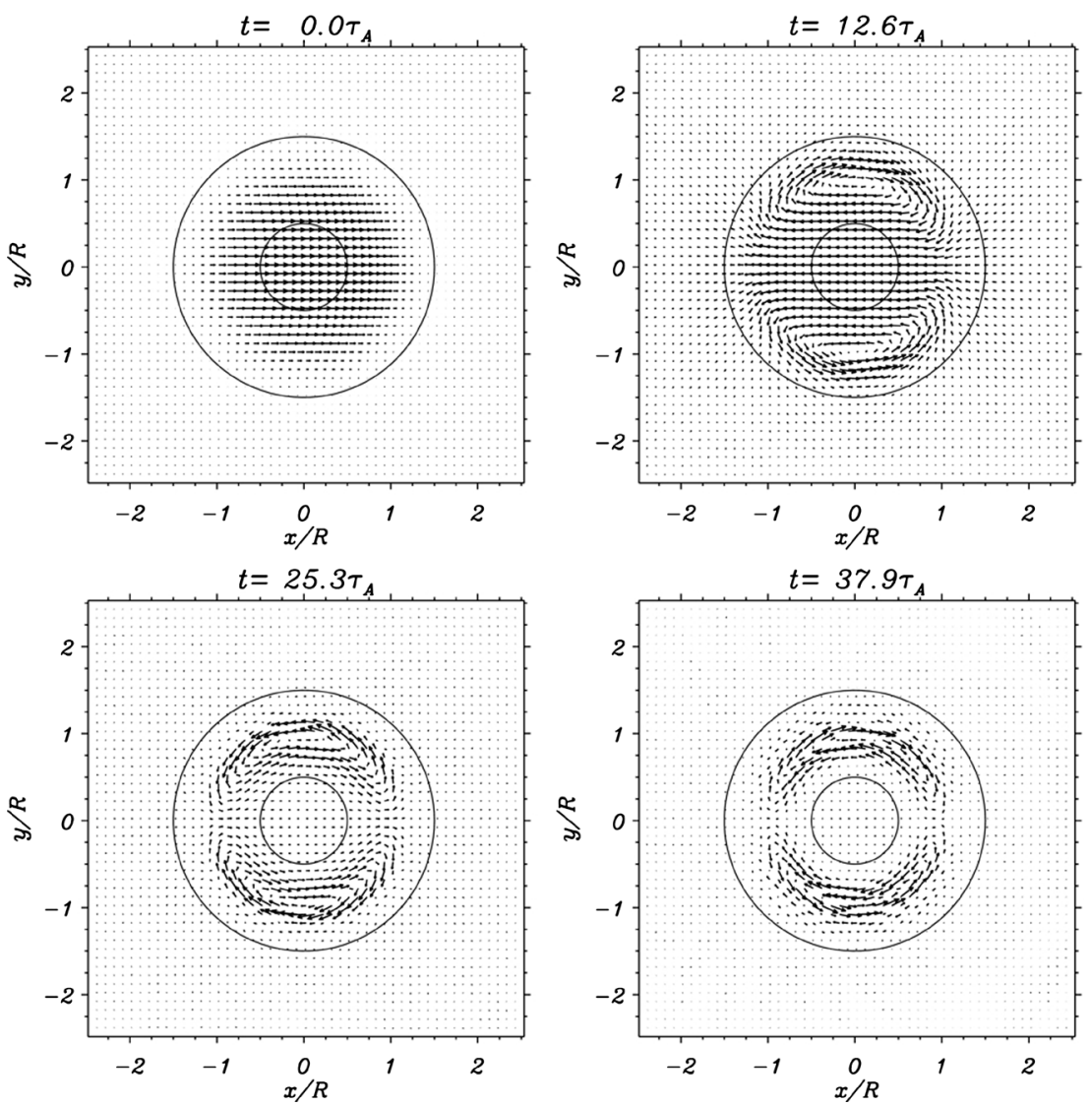

Fig. 23 Snapshots showing the time and spatial evolution of the displacement field (arrows) in a non-uniform plasma cylinder of radius $R$, as the process of resonant absorption develops in a time-dependent numerical simulation. The time evolution is shown in units of transverse Alfvén transit time, $\tau_{\mathrm{A}}$. The annulus represents the non-uniform layer between the internal and external plasmas. From Goossens et al. (2014)

where $f(r)$ is a smoothly varying function for which $f(a+l / 2)=0$ and $f(a-l / 2)=1$, and $l$ is the width of the resonant layer.

Because of the spatial dependence of the density, also the Alfvén speed $V_{\mathrm{A}}(r)$ and Alfvén frequency $\omega_{\mathrm{A}}(r)=k_{z} V_{\mathrm{A}}$ will now depend on $r$. As a consequence, there is a resonance at the point $r=r_{\mathrm{A}}$ between the frequency of the global transverse waves described in Sect. 4, and the local, azimuthal (or torsional with $m=1$ ) Alfvén waves. The necessary condition for the resonance is the collective mode to have a non-zero azimuthal wave number $m \neq 0$ (e.g. the kink mode in a cylinder with a straight field). At this resonant point, the transverse motion in the flux tube is converted into azimuthal motions. This effect is called "resonant absorption" in a solar physics context, but is physically the same effect as "field line resonances" in the magnetospheric context (Sect. 5.1). The temporal/spatial evolution of the resonant absorption process for the kink mode can be traced in the four snapshots of the flow field in Fig. 23. From the initial purely transversal velocity pulse shown in the top left panel, the excitation of the kink mode and its subsequent mode conversion to localised $m=1$ azimuthal motion in the inhomogeneous annulus around the resonant layer can be seen most clearly in the bottom right panel. 
This conversion from transverse motion to azimuthal motion leads to an apparent rapid damping of the macroscopic central flow field that represents the collective MHD mode, even in non-dissipative or slightly dissipative plasmas. The azimuthal motions in the resonant layer of flux tubes cannot be resolved for coronal structures, and they could be damped by phase mixing, Kelvin-Helmholtz instability or kinetic effects.

The effect of resonant absorption was first mathematically described by Chen and Hasegawa (1974) for a plane slab in the context of tokamak physics. It was later suggested by Ionson (1978) that this mechanism could also be responsible for the heating of coronal loops. Early studies of resonant absorption of kink waves in coronal plasma structures modelled by a non-uniform plasma slab include Davila (1987), Steinolfson and Davila (1993), Ofman et al. (1994, 1995). Detailed description of these works and a number of follow-up papers can be found by Goossens et al. (2011). While the plane slab may be a good approximation for field line resonances in the magnetosphere and at some of coronal structures (e.g. streamers, boundaries of coronal holes), the majority of coronal plasma non-uniformities (loops, prominences, plumes, jets) are better modelled with cylinders (see Sect. 4). Early work on implementing this mechanism in cylindrical flux tubes with coronal conditions was done by e.g. Hollweg and Yang (1988), Poedts et al. (1989, 1990).

Following these numerical results, progress was made for resonances in cylindrical configurations by Sakurai et al. (1991), Goossens et al. (1992). In their work, it was assumed that the inhomogeneous layer was very thin $(l / a \ll 1)$, and that the ideal solutions in the homogeneous internal region and external region could be connected by using jump conditions. Later, their expression for the damping rate was simplified by Ruderman and Roberts (2002) for a sinusoidal density profile $\left(f(r)=\frac{1}{2}\left(1-\sin \left(\frac{\pi}{l}(r-a)\right)\right)\right)$. For the plasma cylinder with a straight field the damping rate $\tau$ of a collective MHD mode, measured in units of the oscillation period $P$ is

$$
\frac{\tau}{P}=-\frac{\omega}{2 \pi \gamma}=\frac{2}{\pi} \frac{a}{l} \frac{\rho_{0}+\rho_{\mathrm{e}}}{\rho_{0}-\rho_{\mathrm{e}}} .
$$

This formula was numerically checked for larger $l / a$ with an eigenvalue code by Van Doorsselaere et al. (2004a), who found deviations limited to $25 \%$ for the damping time for a broad range of $l / a$. The analytical theory for resonant absorption in not-so-thin layers was constructed by Hollweg (1990), and further developed in a number of follow-up studies, e.g. Soler et al. (2013). The mathematical approach is based upon the expansion in the Frobenius series near the resonant point. The main conclusion was that the effect does not change principally in the case of a thick resonant layer.

As can be seen in Eq. (40), the resonant damping mainly depends on two parameters: the density contrast $\rho_{0} / \rho_{\mathrm{e}}$ and the relative width of the inhomogeneous layer $l / a$. Transverse oscillations in denser loops are damped more quickly, and oscillating loops with a smoother radial variation of the density profile damp faster. These properties could possibly explain the selective excitation of coronal loops by an initial disturbance, i.e. only some loops in a region oscillate after they have been somehow perturbed (see, e.g., the discussion in Sect. 9.3), while the others experience some outward motion but do not develop an oscillatory motion. This may be because the transverse kink oscillations may be overdamped for some equilibria.

Moreover, indirectly, the damping time is expected to have a linear relationship with the oscillation frequency. This is shown in Fig. 24 for kink oscillations of coronal loops (see Sect. 6.1), where the vertical axis is the damping time and the horizontal axis is the period. As discussed in the previous paragraph, the observed cases are clearly strongly damped (with a low quality factor), but certainly not over-damped. The spread on the data points is 
Fig. 24 The damping times (vertical axis) and periods (horizontal axis) of observed standing kink waves excited by coronal energy releases. Each case is indicated by a discrete symbol. The colour scale shows the quality $\tau / P$ of the oscillations. From Verwichte et al. (2013)

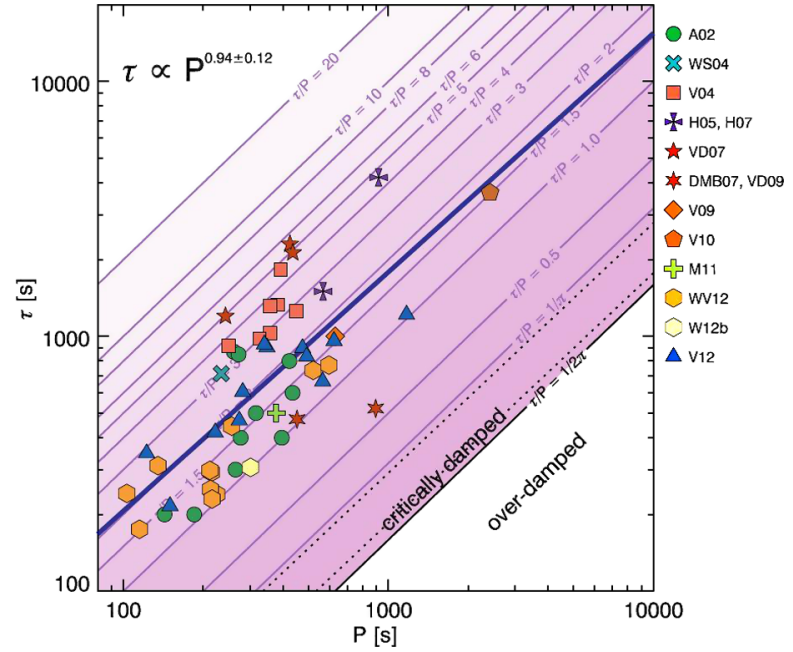

due to the inherently different parameters of the individual loops (different $l / R$ or density contrast).

Another consequence of the linear relationship between the damping time and the period is for the running transverse waves. For propagating transverse waves (see Sect. 7.2), the frequency $\omega$ is fixed by the driver, and instead the equations should be solved for a complex $k_{z}$. Even so, the results are dual (or interchangeable, in the sense that the role of $\omega$ and $k_{z}$ being the free parameter of the problem are apparently swapped), and Terradas et al. (2010) found that the damping length depends directly on the period. This means that the shorter period waves are damped on shorter distances, and that the resonant absorption acts as a low-pass filter for the kink waves excited at the bottom of the corona.

The dependence of the damping times on the properties of the waveguide, e.g. a coronal loop, makes it an interesting observable to use for coronal seismology. Both the period and damping time can be directly observed, and the combination of the information in them with numerical and analytical modelling, has been used by Arregui et al. (2007). They used a numerical model to find narrow ranges for Alfvén transit times in oscillating coronal loops. Moreover, Goossens et al. (2008) constructed an analytical model combining Eq. (26) with Eq. (40) to derive the one-dimensional solution space for the loop parameters $l / a, V_{\mathrm{A}}$ and $\rho_{0} / \rho_{\mathrm{e}}$.

The analytical models have been generalised to more realistic models using numerical simulations, in which the validity of Eq. (40) could be tested. For instance Terradas et al. (2008b) simulated the effect of multi-stranded structure (the oscillating loop consists of a number of closely packed plasma strands or threads) on the damping by resonant absorption. They found that resonant absorption (as a damping mechanism) survives in complicated structures as well. They also found a qualitative agreement, in the sense that the transverse oscillations damp in a few periods. Oscillations in multi-stranded and twisted loops were also studied numerically by Ofman (2009b). It has been shown in that study that the twist could lead to effective coupling between the transverse and longitudinal modes, increasing damping of kink oscillations.

However, there is an inherent problem in understanding observations of damping of oscillations of multi-stranded systems, because the line-of-sight integration of the emission does not allow to identify the sub-resolution, individual strands and their motions (e.g. De Moortel and Pascoe 2012). 
Equation (40) was derived under the assumption that the damping time $\tau$ is much greater than the period $P$. In recent years, the validity of this damping expression (Eq. (40)) has been questioned, since observations (Sect. 6.1) show that the damping time is of the order of the period ( $\tau \gtrsim P$, see also Fig. 24). For example, Pascoe et al. (2013b) found in numerical simulations that the damping is initially described by a Gaussian function $\left(\exp \left(-t^{2} / \tau^{2}\right)\right)$, rather than exponential damping $(\exp (-t / \tau))$ as expected from Eq. (40). This was confirmed by Hood et al. (2013), who quantified the effect analytically. The presence of Gaussian damping has a potential impact on measuring damping times and therefore on seismology with damping times as well.

The main open questions and challenges for the theory of resonant absorption in the context of the corona lie in the generalisation of the theory to more complicated and realistic plasma systems, and in the observational signatures. A more concrete list would be:

- The observed kink oscillations have a large displacement (compared to the loop radius). How does resonant absorption work in such a non-linear regime? How does it compete against the non-linearly excited Kelvin-Helmholtz instability at the edge of the loop (e.g. Terradas et al. 2008a; Antolin et al. 2014)?

- How does resonant absorption work in bundles of loops? How does the global motion transfer to the individual strands, and how do the individual strands dissipate the transverse motion?

- What are the observational signatures of resonant absorption? Can we directly observe the mechanism of resonant absorption taking place, using spectrometers or imaging telescopes?

- What is the role played by the effect of resonant absorption in the undamped kink oscillations, see Sect. 6.1?

\section{Standing Modes of Coronal Plasma Structures}

As in the corona there are both open and close plasma configurations, coronal MHD waves ("local", in contrast with the global coronal waves discussed in Sect. 2) occur in both standing and propagating forms. Wavelengths of standing waves are prescribed by the lengths of resonators, e.g. the length of the oscillating coronal loop. There can be a number of standing longitudinal harmonics. The longest wavelength oscillation is called the global (or fundamental) mode. Usually only the global mode and its second harmonics are detected in coronal observations. Standing MHD oscillations in the corona are truly local, connected with certain loops or other plasma structures. Their wavelengths are determined by the geometrical parameters of the plasma non-uniformities that are essentially smaller than the solar radius. Also, the transverse scale of these non-uniformities is usually much shorter than the longitudinal scale, i.e. the non-uniformities are caused by field-aligned filamentation. Thus, the longitudinal wavelength of coronal standing waves are typically much longer than their transverse scale.

\subsection{Standing Kink Modes}

Transverse oscillations of coronal loops were first observed by Aschwanden et al. (1999) and Nakariakov et al. (1999) in the EUV band, inducing a number of observational studies and theoretical modelling. The oscillations are seen as transverse displacements of bright loops, with periods from a few minutes to several tens of minutes. Different loops have different well defined periods, which suggests that the oscillations have natural frequencies of 
the loop system. The beginning of the oscillation usually coincides with a flare or CME with an epicentre nearby (see Sect. 9.3). The oscillations are almost harmonic. After the excitation, kink oscillations experience a rapid decay, typically fitted with an exponential function with the characteristic decay time equal to 2-4 oscillation periods (although a Gaussian function may be more appropriate, see Sect. 5.5). Hence the quality factor of the oscillations, or the Q-factor, can be defined as $Q=\pi \tau / P$ and is between 6-25. The decay time scales linearly with the period of oscillations (see Ofman and Aschwanden 2002, and also Fig. 24). The strong damping is attributed to the effect of resonant absorption, discussed in Sect. 5.5, however, other mechanisms are possible, such as wave leakage, and phase mixing (see Ofman and Aschwanden 2002, for discussion). The amplitude of the oscillations is typically several Mm, that is several minor radii of the loop (radius of the loop's flux tube cross-section). The typical periods range from a few minutes in short EUV loops to several hours in dense cool filaments of prominences.

The loops of different lengths are observed to have different periods of oscillations. In almost all observed cases only a few periods of oscillations are seen. Occasionally, together with the displacement, periodic variation of the loop brightness is observed. This effect is most likely connected with the periodic variation of the column depth of the oscillating loop segment, caused by its displacement with respect to the LOS (Cooper et al. 2003a; Verwichte et al. 2009).

In the vast majority of cases the oscillations have horizontal polarisation, in other words the loop displacement is parallel to the surface of the Sun. The same polarisation is detected by some occasional quasi-stereoscopic observations (Verwichte et al. 2009). There have not been observational reports of the circular or elliptical polarisation. Usually only the global mode is seen, with the maximum displacement amplitude near the loop top, and only in a few cases higher longitudinal harmonics have been detected (Andries et al. 2009). In all cases, the nodes of the oscillation are observed near the loop footpoints, hence the dense plasma of the chromosphere acts as a rigid wall for the transverse oscillations (c.f. the effect of the ionosphere on the magnetospheric oscillations).

The period of standing kink oscillations is approximately determined as

$$
P_{\text {kink }} \approx 2 L / n_{\mathrm{L}} V_{\mathrm{K}}
$$

where $L$ is the loop length, and $n_{\mathrm{L}}$ is the longitudinal harmonic number that indicates the number of half-wavelengths along the loop (Fig. 26). However, there is a significant deviation, up to $10 \%$, from the equidistant spectrum, i.e., the ratio of the global mode period to the period of the second harmonics is less than 2. This effect is attributed to the interplay between density stratification and flux tube expansion: the Alfvén speed and hence the kink speed at the loop top and the footpoints are different from each other. The global mode with the maximum at the loop top then samples a lower kink speed than the second harmonics that samples the kink speed in the loop legs (see Andries et al. 2009, for detailed discussion).

Very recently a new regime of standing kink oscillations was discovered (Wang et al. 2012; Nisticò et al. 2013; Anfinogentov et al. 2013). It was found out that in addition to the intensively studied large-amplitude rapidly-decaying regime, there are also low-amplitude undamped oscillations, near the very threshold of the available spatial resolution of the observational instruments. Figure 25 shows both the decaying and decay-less regimes for the same loop. Oscillation periods in this new regime are not different from the large-amplitude kink oscillations in the same loop. The amplitude is lower than one minor radius of the loop. All segments of the loop are seen to oscillate in phase and hence the oscillations are standing. Usually several cycles of the oscillation are well seen, with the amplitude remaining constant 

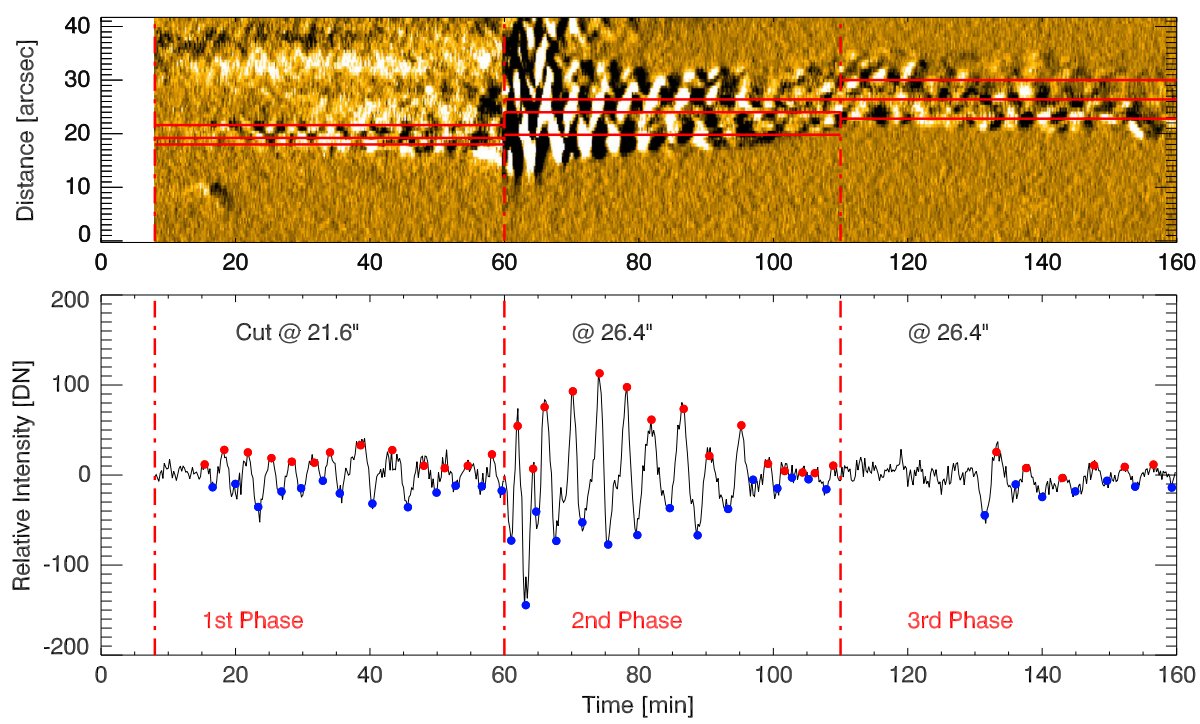

Fig. 25 Kink oscillations of a coronal loop. The top panel shows the time-distance plot made for a slit across the loop near its top. The periodic transverse displacements are clearly seen. The bottom panel shows the zoomed parts of the time distance maps: the red and blue bullet are the maxima and minima positions. The $1 s t$ and $3 r d$ phases correspond to the decay-less low-amplitude oscillations, while the 2nd phase to the large amplitude decaying oscillations excited by a CME. From Nisticò et al. (2013)
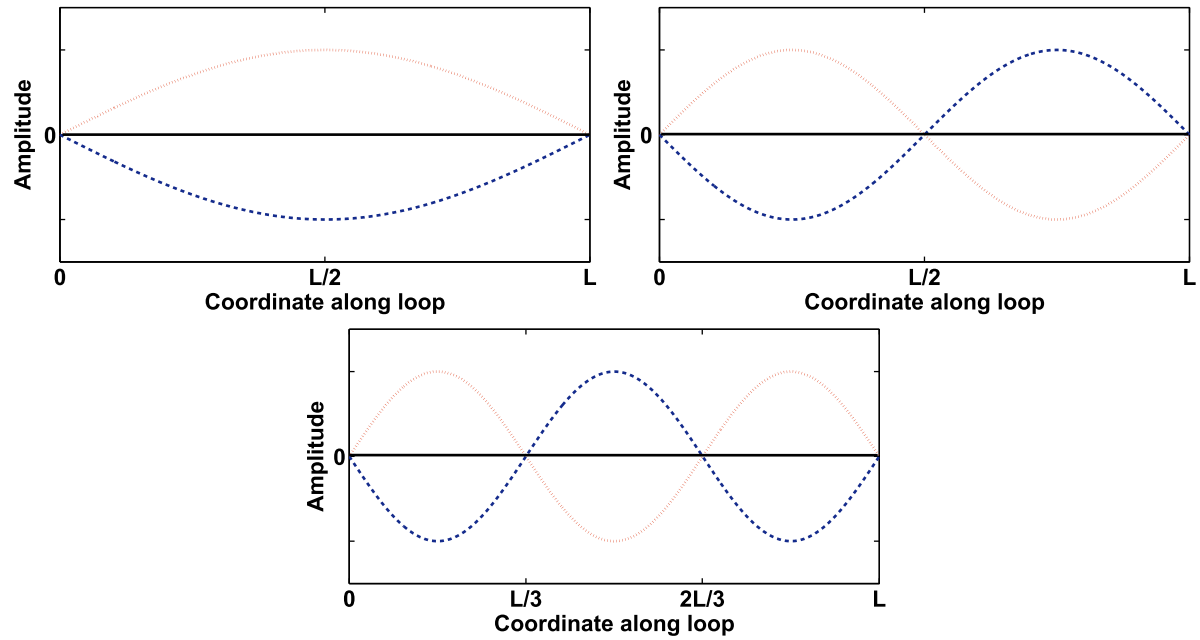

Fig. 26 Displacements of the plasma in the global mode (top left), its second harmonic (top right), and third harmonic (bottom) for a loop of length $L$. The black line shows the position of the undisturbed loop and the red dotted and blue dashed lines indicate the extrema of displacement, corresponding to different phases of the oscillation

or even growing during the observation. There is no observational indication that the oscillation is excited by any bursty energy release. It is very likely that the apparent end of the oscillation is not caused by any decay, but is simply connected with the deterioration of the obser- 
vation conditions. The physical mechanism for the decay-less oscillations is still subject to discussion, while the constant amplitude may result from some balance between persistent external driving, e.g. by granulation motions, and damping, e.g. by resonant absorption.

Despite intensive study, there are still a number of open questions connected with kink modes. More specifically:

- The excitation mechanism in both decaying and decay-less regimes needs to be revealed.

- In the decaying large-amplitude regime, what is the mechanism for the selectivity of the excitation: why some loops gain the large initial displacement while similar loops situated nearby do not?

- Also, what causes the large initial displacement of the loop in this regime? (See discussion in Sect. 9.3.)

- Why is the horizontal polarisation predominantly excited?

- If the rapid decay of kink oscillations is indeed caused by resonant absorption, the transfer of energy from the kink oscillation to the torsional Alfvénic motions needs to be observed at least indirectly, e.g. via the increase in the non-thermal broadening of coronal emission lines.

- It is also unclear whether what we see as an oscillating loop is the actual loop, or it is a bundle of smaller scale plasma threads. For sharp transverse profiles of the plasma in the oscillating loop, resonant absorption efficiency is suppressed, and oscillations of good quality should be observed. But, they are not: in all observed cases the decay time is not longer than a few oscillation periods.

\subsection{Sausage Modes}

In the low- $\beta$ plasma, a sausage ( $m=0$, see Sect. 4) oscillation that is characterised by radial motions of the plasma is difficult to detect. The observations with the line-of-sight perpendicular to the oscillating loop segment give us the same plasma that displaces along the line-of-sight, but does not leave it. Hence, the brightness of the pixel becomes modulated by the oscillations very weakly (Gruszecki et al. 2012). Detection of this mode with imaging telescopes is possible either with the oblique LOS (Antolin and Van Doorsselaere 2013), or in the radio band where the emission may come from a small part of the oscillating volume filled with the non-thermal electrons. Moreover, as sausage modes have periods shorter than the periods of other modes (e.g. 15-20 s), often the required time resolution can be achieved in the radio band only. On the other hand, observations of sausage oscillations in radio impose an additional constraint: the need for the highlighting non-thermal electrons makes it possible only during a solar flare.

The first confident detection of the sausage oscillation was made with NoRH in the microwave band (Nakariakov et al. 2003). Quasi-periodic modulation of the gyrosynchrotron intensity radiation from a flaring loop was found to have the spatial distribution consistent with the theoretically predicted spatial structure of the global sausage mode and its second harmonic. The observed periods were about $16 \mathrm{~s}$ for the global mode and $9 \mathrm{~s}$ for the second harmonic. The modulation depth reached $30 \%$ of the background gyrosynchrotron emission in the flare. Figure 27 shows the microwave image of a flaring loop, the total signal of the gyrosynchrotron intensity emission of the flare, the signals coming from the legs of the loop and from its top, and power spectra of the signals. The top of the loop oscillates with a period of about $16 \mathrm{~s}$. This periodicity is suppressed in the loop legs, where a shorter period, about $9 \mathrm{~s}$, dominates. The longer periodicity corresponds to the global mode, with the maximum signal at the loop top and nodes near the footpoints. The shorter periodicity corresponds to the second harmonics that has a node at the loop top. The presence of the 9-s 

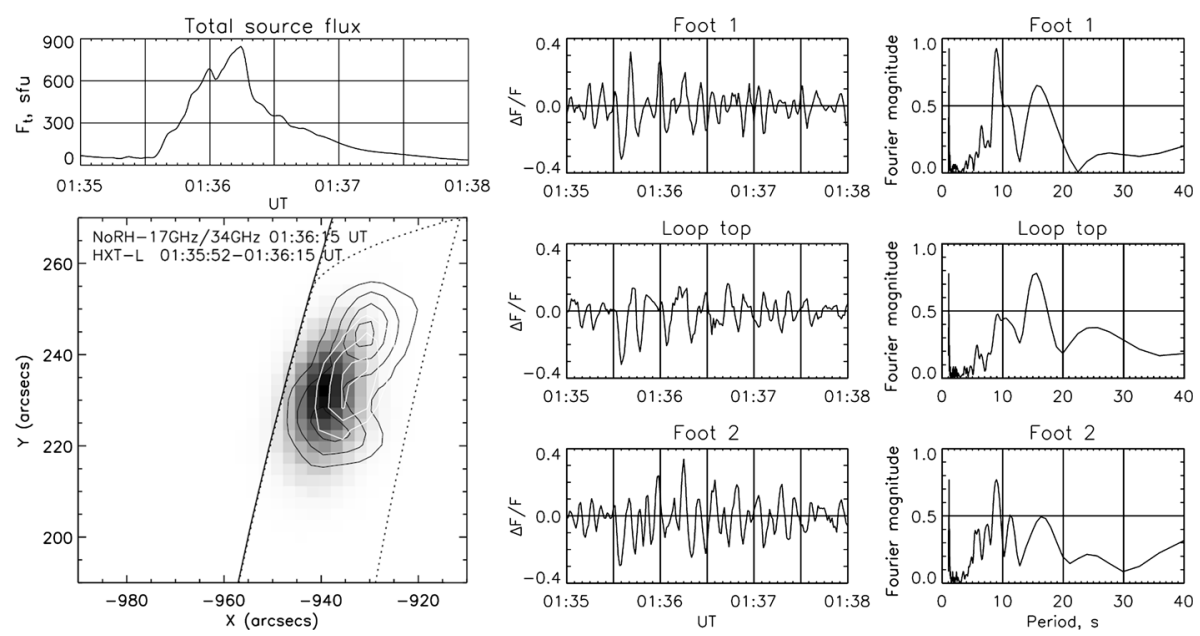

Fig. 27 Left: Radio images of an oscillating flaring loop, made at $34 \mathrm{GHz}$ (filled pixels) and $17 \mathrm{GHz}$ (dotted contour curves). The black contour shows the positioning of the 13.9-22.7 keV hard X-ray sources. The solid line indicates the solar limb. The dotted curves show the heliographic coordinate grid. Centre: The time profiles of the total signal, the modulation depth of the gyrosynchrotron signal at $17 \mathrm{GHz}$ from the Southern leg, the loop top, and the Northern leg of the loop. Right: Fourier power spectra of the modulation depth signals. From Melnikov et al. (2005)

peak in the loop-top signal is attributed to the wide size of the signal integration box that includes a part of the loop legs.

Theoretically, periods of sausage modes of the longitudinal wave numbers $n_{\mathrm{L}}$ are determined by the length $L$ of the oscillating loop,

$$
P_{\text {saus }}^{\text {trap }} \approx 2 L / n_{\mathrm{L}} V_{\mathrm{ph}},
$$

where $V_{\mathrm{ph}}$ is the phase speed that is in the range $V_{\mathrm{A} 0}<V_{\mathrm{ph}}<V_{\mathrm{Ae}}$. The phase speed decreases with $n_{\mathrm{L}}$ because of geometrical dispersion, which makes the spectrum highly uneven: the period of the second harmonics is longer than half of the global mode period. This property is confirmed by both observations (Inglis et al. 2008) and numerical modelling (Inglis et al. 2009). This effect is attributed to the dispersion only, and not connected with the plasma stratification. Even for finite- $\beta$ plasmas that can appear in flaring loops, the sausage mode period is independent of the plasma temperature (Inglis et al. 2009).

In the leaky regime, the dependence of the sausage mode period on the loop length decreases, and for sufficiently long and thin loops the period becomes independent of the loop length (e.g. Cally 1986; Kopylova et al. 2007). Theoretical estimations show that the period becomes determined by the transverse transit time across the loop, $P_{\text {saus }}^{\text {leaky }} \approx \pi a / V_{\mathrm{A} 0}$ (see, e.g. Cally 1986; Terradas et al. 2005, for the analytical calculations for a step-function transverse profiles of the equilibrium parameters). Numerical simulations showed that the period depends also on the steepness of the profile (Nakariakov et al. 2012). It was shown that the sausage mode may persist long enough to be observable in high density contrast structures, even though it is leaky (Vasheghani Farahani et al. 2014).

The periodic modulation of the gyrosynchrotron emission is produced by a sausage mode via the perturbation of the magnetic field strength and plasma density in the emitting plasma in the loop. Simultaneously, periodic variation of the hard X-ray and gamma-ray emission can be observed at the loop footpoints. The nature of the high-energy radiation modulation 
is believed to be connected with the variable magnetic mirror effect-the Zaitsev-Stepanov mechanism (Zaitsev and Stepanov 1982, see Sect. 11.3 for details). Flaring loops have wider cross-sectional areas near the top. Thus, the loop legs act as magnetic mirrors for nonthermal electrons with sufficiently large pitch angles. The sausage oscillation changes the cross-sectional area near the loop top, allowing some non-thermal electrons to reach the footpoints, interact with the dense plasma, and emit high-energy radiation by the bremsstrahlung mechanism.

Recently, short period standing sausage oscillations were detected in the quasi-periodic wiggling of zebra-patterns (Yu et al. 2013). Zebra-patterns are sets of almost-parallel stripes superposed on the dynamical spectra of type IV radio bursts associated with CMEs and solar flares. The bursts are characterised by a broadband continuum emission generated by an unstable loss-cone distribution of super-thermal electrons accelerated in a flare. Zebra-patterns are associated with the coherent generation of upper hybrid waves at the locations of double plasma resonances in a non-uniform plasma, where $N \Omega_{\mathrm{e}}=\sqrt{\Omega_{\mathrm{e}}^{2}+\omega_{\mathrm{pe}}^{2}}$ with $N$ being an integer. Variations of the plasma density and magnetic field produced by sausage oscillations changes the double plasma resonance conditions and spatial locations, that results in the periodic variation of zebra-pattern stripes in the spectrum.

There are several open questions regarding sausage oscillations.

- What are the properties of this mode in EUV and soft X-ray loops, and why have these modes not been confidently identified in these bands?

- In relation to sausage mode modulation of the magnetic mirror ratio, this still requires detailed modelling for estimating its efficiency and forward modelling of the observables, which is the prediction of physical parameters open to observations from existing models.

- Can sausage oscillations accelerate charged particles by, e.g., the betatron mechanism, and what is efficiency and observational manifestation of this effect?

\subsection{Longitudinal Modes}

Longitudinal oscillations in coronal loops were discovered as periodic Doppler shifts recorded with the SOHO/SUMER instrument (Wang et al. 2002), and hence are often called SUMER oscillations. Typical periods range in 7-31 min; typical decay times are usually one or two oscillation periods; and typical amplitudes are $50-100 \mathrm{~km} \mathrm{~s}^{-1}$ that makes the relative amplitude up to $30 \%$ of the sound speed (see Wang 2011, for a comprehensive review). A few observations when the periodic Doppler shift was observed simultaneously with the periodic variation of the intensity revealed a quarter-period phase shift $(\pi / 2)$ between them. Such a phase shift is consistent with the basic theory of standing acoustic waves. Indeed, in the global longitudinal oscillation of a loop of length $L$, the spatial distribution of the velocity perturbation along the loop is $V_{\|} \propto \cos (\pi s / L) \cos (\omega t)$, where $s$ is the coordinate along the loop, $s=0$ coincides with the loop top. The corresponding density perturbation is $\tilde{\rho} \propto \sin (\pi s / L) \sin (\omega t)$. As the sine and cosine are phase-shifted by quarter the period, the observation is consistent with the standing acoustic (or, more rigorously, slow magnetoacoustic) mode.

Recently, compressive oscillations with the properties similar to SUMER oscillations, have been detected in the microwave and EUV data (Kim et al. 2012). Variations of the thermal radio emission and of the EUV radiation in a hot plasma loop were found to have the same rapidly-decaying oscillatory pattern with a 12-min period (see Fig. 28). The observed parameters of the oscillations are consistent with SUMER oscillations. Oscillations resembling SUMER oscillations, but with much shorter periods, about $2 \mathrm{~min}$, and decay times about 2.5-6 min were found in the soft X-ray band (Ning 2014). 

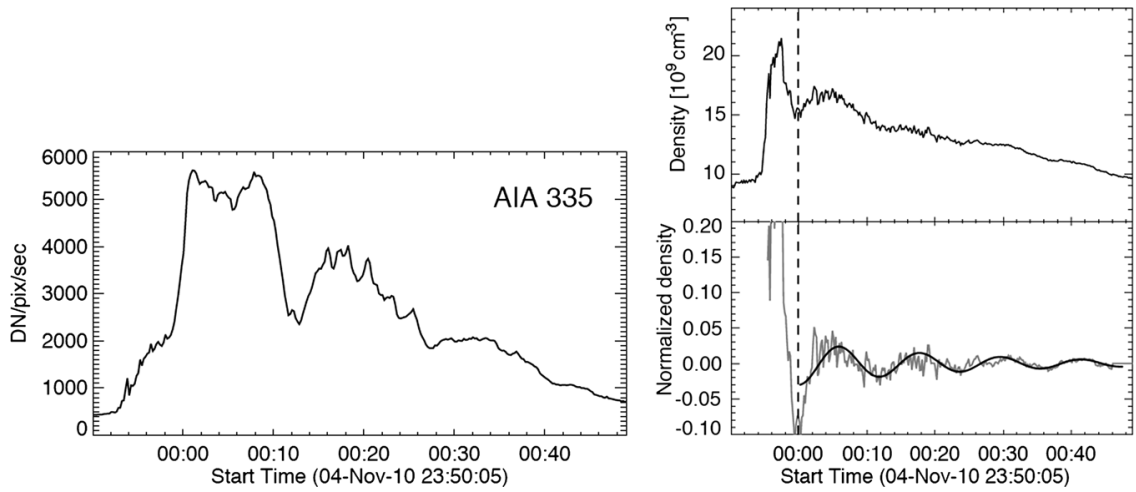

Fig. 28 Example of a standing longitudinal oscillation in a hot coronal loop, observed simultaneously in the EUV and microwave bands. Left: Time profile of maximum counts obtained at the AIA $335 \AA$ channel in a flare loop. Right: Time profile of the electron number density derived by electron temperature and the temperature brightness at $17 \mathrm{GHz}$ (top panel), and a best fitting of the detrended and normalised profile with an exponentially decaying harmonic curve (bottom panel). The vertical dashed line shows the instant of time after which the microwave emission is free-free. From Kim et al. (2012)

As it has been already mentioned above, SUMER oscillations are interpreted as standing longitudinal (or slow magnetoacoustic) waves (Ofman and Wang 2002). The oscillation period is determined by the length of the loop $L$ and by the tube speed in the loop

$$
P_{\text {long }} \approx 2 L / n_{\mathrm{L}} V_{\mathrm{T} 0} \text {. }
$$

As in a low- $\beta$ plasma the tube speed is almost equal to the sound speed, the period of longitudinal oscillations is often estimated with the use of the sound speed $V_{\mathrm{s} 0}$, instead of the tube speed $V_{\mathrm{T} 0}$. However, accounting for the finite- $\beta$ effect, it became possible to use SUMER oscillations for the estimation of the absolute value of the magnetic field and Alfvén speed in the oscillating loops (Wang et al. 2007). Because of the long periods the oscillating plasma is well collisional, and hence these waves are not subject to Landau damping. Decay of the oscillations was shown to be consistent with damping caused by thermal conduction (Ofman and Wang 2002).

Mechanisms for the excitation of standing longitudinal oscillations in the corona are still not clear. An impulsive energy release somewhere in the loop or near its footpoint easily excites the global longitudinal mode or its second harmonics (Nakariakov et al. 2004a; Taroyan et al. 2005). The most effectively excited longitudinal harmonics are prescribed by the position (or positions) of the energy deposition. If the energy is released near the loop top, or simultaneously at both its footpoints, most of the energy goes to the second standing harmonics. If the source is localised near one footpoint only, or there is a strong asymmetry of the footpoint sources, the global acoustic mode is excited. The reason for such an impulsive energy release can be magnetic reconnection in a flare, e.g. direct heating of the plasma, or by bombardment of the chromosphere and photosphere by non-thermal electrons. However, relationship of the SUMER oscillation occurrence with the strength of the energy release is absent. For example, of 27 events of SUMER oscillations, only 6 were seen to be associated with flares stronger than the C-class (Wang 2011). The majority of oscillation events are associated with micro-flares or localised mild increases in hard X-ray emission. Moreover, SUMER oscillation events show a high recurrence rate. More than a half of the detected events are recurring with a rate of 2-3 times within two hours, which 
happen at the same place and manifest identical periods and initial Doppler shifts of the same sign. Perhaps, the oscillations are excited not by the localised impulsive increase in the plasma temperature or gas pressure, but a field-aligned pulse of the hot plasma.

Advanced numerical simulations of longitudinal oscillations (Nakariakov et al. 2004a) that accounted for the peculiarities of the radiation and heating functions, demonstrated the presence of another, decay-less regime of these oscillations. Decay-less oscillations in a dissipative medium are known as auto-oscillations or self-oscillations. Properties of autooscillations, e.g. the amplitude, are prescribed by the balance between the energy deposition and damping, and are independent of the initial perturbation. A similar effect, periodic variation of the loop temperature and density due to the evaporation-condensation cycle, accompanied by subsonic longitudinal flows, was found in hydrodynamic simulations of coronal loops with time-independent non-uniform heating (Müller et al. 2004). The existence of the limit cycle regime, corresponding to auto-oscillations, was revealed. However, the auto-oscillatory regime has not been identified in observations yet.

Numerical simulations carried out by Taroyan et al. (2005) in homogeneous and stratified loops demonstrate that standing acoustic waves can be excited by an impulsive heat deposition (e.g. a pressure disturbance associated with the injections of hot plasma) at the chromospheric footpoint of a loop. The excitation of the fundamental mode occurs if the duration of the pulse matches the fundamental harmonic. The amplitude of the oscillations results to be proportional to the amplitude of the pulse, which is immediately followed by a standing wave consistent with the observations of the longitudinal oscillations in a hot loop done with SOHO/SUMER. The amplitude is determined by the amount of the released energy. Furthermore, these results have a relevant implications in coronal seismology: the duration of the heat release is equal to the period of the fundamental mode, the velocity amplitude provides a lower estimate for the amount of heat released at the chromospheric footpoint of the loop. Longitudinal oscillations could also be excited by injections of fieldaligned sub-sonic flows at the footpoints (e.g. Ofman et al. 2012). Selwa et al. (2007) found that the excitation efficiency depends on the loop curvature and on the localisation of the initial pulse in the directions across the loop axis, and that in the realistic case of a curved waveguide the excitation of a fast magnetoacoustic wave in the external medium may be important.

In the low- $\beta$ plasma, slow magnetoacoustic waves propagate along the field at the speed about the sound speed. However, finite- $\beta$ effects may be important in hot flaring loops. In particular, it was shown that for a plasma where the sound speed is only a few times lower than the Alfvén speed, the highest value of the slow wave group speed in the perpendicular direction reaches $25 \%$ of the sound speed. For a broad range of parameters, the highest perpendicular group speed corresponds to the propagation angle of $25^{\circ}-28^{\circ}$ to the magnetic field (Nakariakov and Zimovets 2011). Thus, in a magnetoacoustic waveguide that is, for example, formed by two parallel rigid planes anchoring the magnetic field lines perpendicular to the planes, slow waves can propagate across the field in the direction parallel to the planes by a series of footpoint reflections. In observations, this effect may be associated with the progression of a solar flare along the magnetic neutral line in large, two-ribbon flares (Nakariakov and Zimovets 2011, see also Sect. 11.4).

The open questions posed by longitudinal oscillations are the following.

- Why do the majority of observational detections of this mode come from hot loops?

- What is the main driver of the oscillation, if in about a half of observed events the longitudinal oscillation is seen without a noticeable flare?

- A connected question is, what is the reason for the high excitation rate of the oscillations by small flares? 
- The apparent recurrence of the oscillation events suggests selectivity of the excitation mechanism, but what is the physical reason for the selectivity?

- What is the effect of the nonlinearity associated with the large oscillation amplitude, and how can it be detected observationally?

- Regarding observations, it is necessary to look for examples of shorter period oscillations, and to study the scaling of the oscillation period and decay time with the loop length and the plasma temperature.

- It is also of interest to search for the resonant excitation of longitudinal oscillations by umbral and penumbral sunspot oscillations in the loops anchored in sunspots.

So far, the majority of theoretical models of longitudinal oscillations were restricted to the infinite-field approximation: only the field-aligned flows were considered. This approach is more suitable to the waves that are plane in the transverse direction. However, in coronal loops and other plasma non-uniformities longitudinal waves are in an entirely different regime. Their transverse wavelength is much shorter than the longitudinal wavelength. This difference results in the longitudinal phase speed being the tube speed, rather than the sound speed, even without any wave-guiding non-uniformity (Roberts 2006). In a low- $\beta$ plasma this effect does not significantly change the longitudinal speed, justifying the use of the infinite-field approximation for the determination of the resonant properties of plasma loops. However, it may be important for nonlinearity, dissipation and observability, and hence needs to be considered in detail (see, e.g. Afanasyev and Nakariakov 2015). Likewise, identification of the auto-oscillatory regime would be interesting for revealing the plasma heating function.

\section{Propagating MHD Waves in Coronal Plasma Structures}

There are also various propagating waves detected in the corona. Below we describe several types of propagating waves and their interpretation in terms of MHD wave theory in a plasma cylinder, given in Sect. 4.

\subsection{Propagating Longitudinal Waves}

One of the first wave phenomena detected in the solar corona with sufficient spatial and time resolution, was the slowly-propagating waves of EUV emission disturbances in polar plumes (Ofman et al. 1997, 1999; DeForest and Gurman 1998), in the inter-plume regions (Banerjee et al. 2001), and near footpoints of long coronal loops and fan-like structures (Berghmans and Clette 1999; Robbrecht et al. 2001). Comprehensive reviews of these phenomena can be found by Nakariakov (2006), De Moortel (2009). Here, we introduce the term "slowlypropagating waves" to highlight their difference from the rapidly propagating waves of EUV emission disturbances, described in Sect. 7.4. The waves are seen as upwardly propagating quasi-periodic intensity variations in EUV and soft X-ray bands. They are well-identified as periodic diagonal stripes in the time-distance map (see Fig. 29). The direction of the wave propagation coincides with the direction of the plasma filamentation and hence with the direction of the magnetic field. Fixing the spatial coordinate, i.e. choosing a certain value at the horizontal axes in panels b or c of Fig. 29, one gets a time signal and can determine its periodicity. The periodicity is typically well-defined and stable, and hence the signal is quasi-monochromatic. The period ranges from a few to several minutes. 

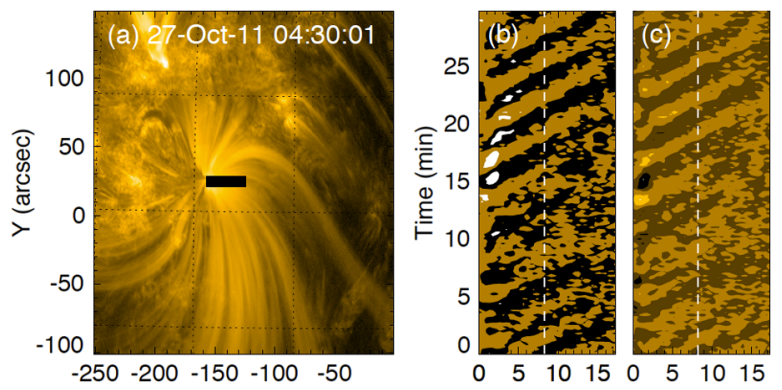

Fig. 29 Example of a propagating quasi-periodic longitudinal oscillation in a coronal plasma fan. (a) SDO/AIA $171 \AA$ image of active region NOAA 11330 observed on 27 October 2011 at 04:30:01 UT. A cut that was taken to make the time-distance plot is indicated with a black bar. (b) Time-distance plot of the running difference, made along the cut. It covers about 10 cycles of the propagating features. (c) The same time-distance plot but made with the background-subtraction method. The diagonal stripes in panels (b) and (c) indicate the outwardly propagating waves of the EUV intensity. From Yuan and Nakariakov (2012)

It is observed that propagating slow waves are strongly damped. Typically (e.g. De Moortel et al. 2002), it was found that waves with a period of 5 minutes reach in coronal loops or fan-like plasma structures in active regions the height of only $10 \mathrm{Mm}$, which is $1-2$ wavelengths along the wave path. Recently, it was found that long-period (up to 25 minutes), propagating slow waves can reach heights up to $150 \mathrm{Mm}$ (Krishna Prasad et al. 2012), and seem to be omnipresent in the solar corona. Due to the quality factor that is nearly independent of the period (Krishna Prasad et al. 2014), the long-period waves can reach much greater heights before they are damped.

The damping of these compressive waves is thought to be mainly due to classical dissipative processes (Ofman et al. 1999, 2000; Nakariakov et al. 2000), in particular thermal conduction (De Moortel and Hood 2003), and coronal loop/field line expansion (De Moortel and Hood 2004). However, the recent observational results obtained by Krishna Prasad et al. (2014) question whether these damping mechanisms are truly at work, at least in polar plumes, because the frequency dependence of the quality factor of the observed waves does not agree with the theoretically predicted dependence. Also, it should be noted that observational limitation of the intensity fluctuations with height could lead to apparent wave damping, in addition to the physical damping.

\subsection{Propagating Kink Waves}

As described in Sect. 6.1 the post-flare standing kink oscillations were first detected in coronal loops by TRACE (Aschwanden et al. 1999; Nakariakov et al. 1999), but it was not until several years later that reports were made of propagating kink waves in the corona by Tomczyk et al. (2007) using data from the Coronal Multi-Channel Polarimeter (CoMP). ${ }^{2}$ Typical periods are from several minutes to tens of minutes, with a preference for 5 minutes. The primary reason for the delay of this discovery was the small amplitude nature of propagating kink waves $\left(\approx 1 \mathrm{~km} \mathrm{~s}^{-1}\right)$. A kink wave with a maximum velocity amplitude of $1 \mathrm{~km} \mathrm{~s}^{-1}$ and a period of 5 minutes will only displace the loop by at most $48 \mathrm{~km}$, which is nearly an order of magnitude smaller than the $365 \mathrm{~km}\left(0.5^{\prime \prime}\right)$ pixel size of TRACE. However, the advanced

\footnotetext{
${ }^{2}$ The rapidly-propagating waves of emission intensity, detected with the Solar Eclipse Coronal Imaging System (SECIS) by Williams et al. (2002) and Katsiyannis et al. (2003) could also be produced by kink waves by the variation of the apparent column depth (Cooper et al. 2003a,b).
} 
Doppler velocity resolution of the ground-based CoMP, which was primarily designed to make accurate off-limb measurements of the Zeeman effect, offered the first opportunity to study these propagating low amplitude and broadband frequency kink waves. Importantly, these waves were found to be ubiquitous and were not seen to be driven by any large scale energy release events such as flares and CMEs, as is the case with coronal loop standing kink waves observed with TRACE and latterly SDO/AIA. We need to point out that effects of phase mixing, line of sight integration, and instrumental effects (resolution, scattering) could account for the apparent low amplitudes of the observed kink waves.

Initially Tomczyk et al. (2007) interpreted these propagating waves as Alfvén waves, because they have phase speed close to the expected Alfvén speed $\left(\approx 1000 \mathrm{~km} \mathrm{~s}^{-1}\right)$, are strongly guided by the coronal magnetic field and are almost incompressive in character (with intensity variations of $\Delta I / I<3 \times 10^{-3}$ ). However, in contrast to its excellent Doppler velocity resolution, the spatial sampling of CoMP is a relatively coarse $4.5 \mathrm{Mm}$. It was demonstrated by Van Doorsselaere et al. (2008a) that in the flux tube geometry of coronal loops, only kink waves would display the collective behaviour observed with CoMP on such spatial scales. Essentially, only fast magnetoacoustic waves guided by a field-aligned plasma non-uniformity can propagate along the field and have the transverse size (about $8 \mathrm{Mm}$ ) much shorter than their longitudinal wave lengths (about $300 \mathrm{Mm}$ ). In contrast, torsional Alfvén waves are not collective modes, existing independently on each magnetic surface (see also discussion in Sect. 4). Consequently, they can have different amplitudes, phases and frequencies on each surface and would simply not display the coherence observed over such spatial scales by Tomczyk et al. (2007), especially after Alfvénic perturbations of different magnetic surfaces get out of phase after some time.

Interestingly, after the launch of Hinode in 2006, its highly effective EUV imaging spectrometer (EIS) also detected low amplitude kink waves $\left(\approx 1 \mathrm{~km} \mathrm{~s}^{-1}\right)$ in coronal loops that were not seen to be generated by either flares or CMEs (Van Doorsselaere et al. 2008c; Erdélyi and Taroyan 2008). Unfortunately, limited by the narrow spectroscopic slit of EIS, these authors could not observe the loop in its entirety, and were therefore not able to establish if the kink waves were propagating or standing. In that sense, the larger field of view of CoMP had the advantage of determining whether the kink waves are propagating or not, but certainly the presence of non-flare and non-CME generated low amplitude kink waves was confirmed independently by both instruments. Subsequently, propagating kink waves have also been detected with the high resolution EUV imager AIA onboard SDO (launched in 2010) in coronal loops, coronal holes, quiet Sun regions and polar plumes (see e.g., McIntosh et al. 2011; Thurgood et al. 2014). Now there have even been multi-instrumental studies of this phenomena with CoMP and SDO/AIA (Threlfall et al. 2013), and also the ultra-high resolution EUV imager Hi-C with SDO/AIA (Morton and McLaughlin 2013).

As standing kink waves in coronal loops were observed to exhibit rather strong damping, it was natural to assume that the same would also be true of propagating kink waves. Observations of kink waves propagating along coronal loops, made with CoMP (Tomczyk and McIntosh 2009) indicate that it may be indeed the case. Using this as motivation, Terradas et al. (2010) calculated that the damping length of kink waves due to resonant absorption should be proportional to the period. Hence, the effect of resonant absorption is to cause longer damping lengths for lower frequencies, i.e., the damping effect of a resonant layer acts like a low-pass filter for propagating kink waves (see discussion in Sect. 5.5). Therefore, the broadband frequency information contained in the CoMP data offered an excellent opportunity to test this model. Verth et al. (2010) used the same data set as Tomczyk and McIntosh (2009) and found that there was qualitative agreement for the estimated quality factors for both standing and propagating waves in coronal loops, as would be expected for the resonant absorption model. 
In highly dynamic regions of the corona propagating kink waves have also been observed in the presence of sub-Alfvénic and even Alfvénic flows. Using Hinode/XRT, Cirtain et al. (2007) observed kink waves in a polar coronal hole X-ray jet, which had two distinct flow components, one near the Alfvén speed $\left(\approx 800 \mathrm{~km} \mathrm{~s}^{-1}\right)$ and another near the sound speed $\left(\approx 200 \mathrm{~km} \mathrm{~s}^{-1}\right)$. Modelling this particular event Vasheghani Farahani et al. (2009) deduced that in the observationally determined range of parameters, the kink waves were not found to be subject to either the Kelvin-Helmholtz instability or the negative energy wave instability. Hence, they determined that the kink waves must have been excited at the source off the jet and were actually observed as they were propagating upwards. Such propagating kink waves associated polar coronal hole EUV jets have since been observed with SDO/AIA (e.g., Morton et al. 2012; Chandrashekhar et al. 2014).

There is a number of open questions connected with propagating kink waves:

- Is there a link between propagating kink waves and standing undamped kink oscillations?

- What is the driver of the propagating kink waves? What does determine the horizontal size of the wave?

- The very existence of the propagating kink waves requires presence of field-aligned nonuniformities of the plasma density. Can those non-uniformities be identified in the diffuse corona, where the waves are detected?

- What is the wave polarisation, plane, elliptical or circular?

\subsection{Transverse Waves in Coronal Streamers}

Propagating transverse waves of much longer periods and wavelengths are observed in coronal streamers. Coronal streamers, also called helmet streamers, are large cap-like bright structures with long pointed peaks extended outwards the Sun. Morphologically, these coronal structures consist of a loop anchored at the surface of the Sun, above which the externally lying magnetic field lines form a cusp and a radially-stretched current sheet. The current sheet is surrounded by a high-density plasma sheet or slab, also called a streamer stalk. The magnetic configuration of helmet streamers clearly resemble the nightside magnetosphere with the magnetotail (Sect. 8).

Quasi-periodic transverse displacements of a streamer stalk (see Fig. 30), excited by a CME development were recently observed with coronagraphs (Chen et al. 2010; Kwon et al. 2013) at the heights above one solar radius from the solar surface. The CME impact acts as an initial perturbation for the transverse wave guided by the plasma non-uniformity. The oscillation periods were estimated in the range from $60 \mathrm{~min}$ to $150 \mathrm{~min}$. There is some evidence of the increase in the oscillation period with height. The perturbation propagates along the streamer's current sheet with the apparent phase speed of about $300-500 \mathrm{~km} \mathrm{~s}^{-1}$ (Chen et al. 2010). Different studies showed that the phase speed decreases with height. Also, the oscillation decays very rapidly, with the decay time approximately equal to the oscillation period. The periods and damping times measured at different heights were found to be highly correlated and scale linearly with each other (Kwon et al. 2013). The amplitude of the displacement is $50-100 \mathrm{Mm}$ (up to $20 \%$ of the solar radius). Excitation of the visible transverse motion in streamers is observed in only a very small fraction of the CME deflection events. The CMEs causing the transverse waves have high phase speed, over $1000 \mathrm{~km} \mathrm{~s}^{-1}$.

Observations of transverse waves guided by streamers are still very rare and require targeted search and analysis. The lack of statistically significant observational information about this phenomenon makes its theoretical modelling rather speculative. However, the rareness of these transverse coronal waves poses questions concerning their excitation and its selectivity. 
Fig. 30 Transverse motion of a coronal streamer stalk, as an aftermath of the CME impact, seen in the snapshots of coronal images taken in the white light during an artificial eclipse with $\mathrm{SOHO} / \mathrm{LASCO}-\mathrm{C} 2$. The white circle in the top-left corner of the images indicates the solar limb. The wavy displacement propagating outwards at the Southern slope of the bright streamer is the transverse waves. From Chen et al. (2010)
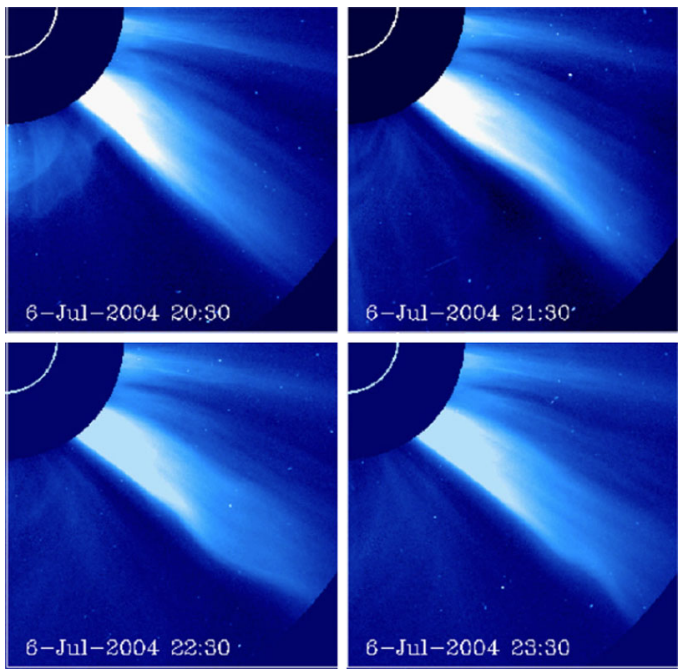

Fig. 31 Numerical simulation of an impulsively generated fast magnetoacoustic wave train propagating along a coronal loop with a density contrast ratio of 5.0 and profile steepness power index equal to 8. Upper panel: the characteristic time signature of the wave train. Lower panel: wavelet transform analysis of the signal, demonstrating the characteristic "crazy" tadpole wavelet signature. From Nakariakov et al. (2004b)
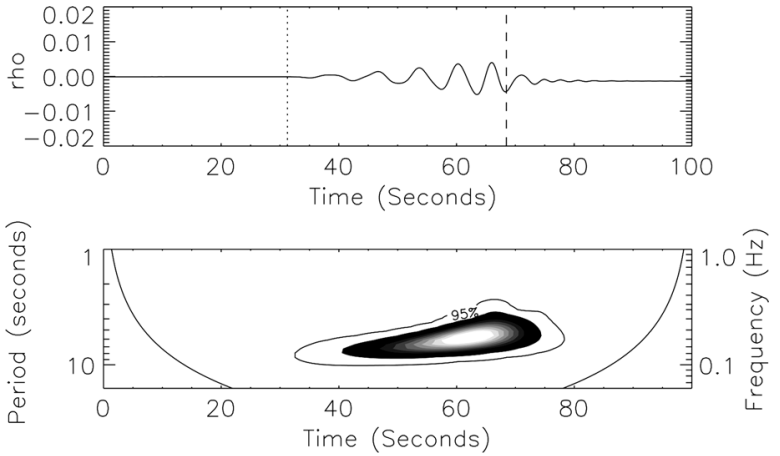

\subsection{Rapidly-Propagating Wave Trains}

Magnetoacoustic modes of plasma non-uniformities are subject to geometric dispersion (see Sect. 4). In the low- $\beta$ plasma (typical for the corona and magnetosphere), dispersion of fast waves is significant. Hence, a broadband initial fast magnetoacoustic perturbation develops in a quasi-periodic wave train as different spectral components propagate at different phase and group speeds because of the dispersion. It has been theoretically predicted that fast magnetoacoustic waves guided by solar coronal plasma structures, can form quasi-periodic wave trains, (Nakariakov et al. 2004b, and references therein). The analysis of the group speed dependence on the longitudinal wave number shows that the long-wavelength spectral components propagate faster than the medium- and short-wavelength ones. This suggests that an impulsively generated fast wave train could have a characteristic signature with three distinct phases: the periodic phase, the quasi-periodic phase and the decay phase (Roberts et al. 1983). Nakariakov et al. (2004b) simulated the formation of the quasi-periodic wave trains in field-aligned plasma slabs with transverse profiles of the plasma density of different steepness. Fast magnetoacoustic wave trains were shown to have a characteristic "crazy" tadpole wavelet (or dynamical Fourier) signature, see Fig. 31. These signatures were detected in the observations in the visible light, and also in decimetric radio bursts (Mészárosová 

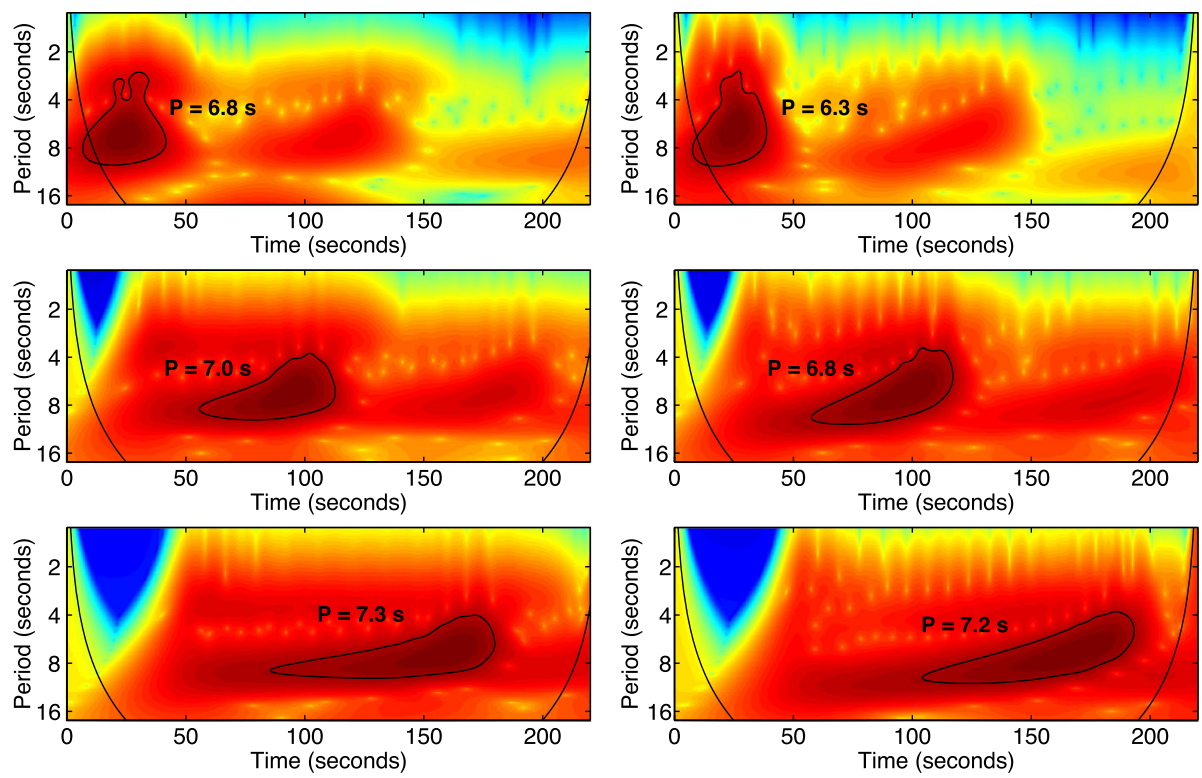

Fig. 32 Wavelet spectra ("the crazy tadpoles") of fast magnetoacoustic wave trains at three different detection points with the increasing distance from the broadband source (upper, middle, and lower panel, respectively). In the left column, the results for the density slab are shown, whereas the results for the Harris current sheet can be seen in the right column. From Jelínek and Karlický (2012)

et al. 2009). The effect of the amplitude and frequency modulation of coronal fast wave trains is most pronounced if the initial excitation is sufficiently broadband (Nakariakov et al. 2005). Narrowband initial perturbations are not affected by dispersion and remain quasi-monochromatic, see Sect. 7.2.

The so-called spikes (defined as a group of very short and narrowband radio bursts) are observed during the solar flares. Karlický et al. (2011) found the signatures typical for fast magnetoacoustic wave trains formed by dispersion, in decimetre radio spikes. They concluded that these fast wave trains were associated with fast magnetoacoustic waves propagating along the dense plasma slab surrounding the global current sheet (current layer) in turbulent reconnection outflows. These waves modulate the process of acceleration of electrons and hence the generation of plasma and electromagnetic waves, resulting in the radio spikes. Jelínek and Karlický (2012) and Mészárosová et al. (2014) performed a more extended and detailed study of fast sausage wave train dynamics in a plasma slab with a current-sheet, see Fig. 32. The formation of fast wave trains was recovered. It was suggested that these wave trains could modulate the radio fluxes (and even UV fluxes) in various locations in the current-sheet.

A similar effect has also been observed in radio fiber bursts (Karlický et al. 2013), that are fine spectral structures observed in broadband radio emission of solar flares, characterised by fast frequency drift. Their wavelet spectra were found to contain tadpole-like features, as shown in Fig. 31. The frequency drift in the tadpole head is the same as the frequency drift of fiber bursts. This effect can be associated with a fast sausage magnetoacoustic wave train propagation upwards in a stratified atmosphere. The wave train modulates the radio emission produced by super-thermal electrons accelerated and trapped in the flare site. 
Fig. 33 (a) The AIA

field-of-view in $171 \AA$ displaying the active region AR11227 and the flare epicentre.

(b)-(d) Running difference images illustrate the fast wave trains. The wave fronts of three distinct wave trains are marked by arrow groups in different colours. The solid lines (L1-L5) show the funnel. The dashed arcs concentric at the flare epicentre, have the radii of $100 \mathrm{Mm}$, $200 \mathrm{Mm}$ and $300 \mathrm{Mm}$, respectively. From Yuan et al. (2013)
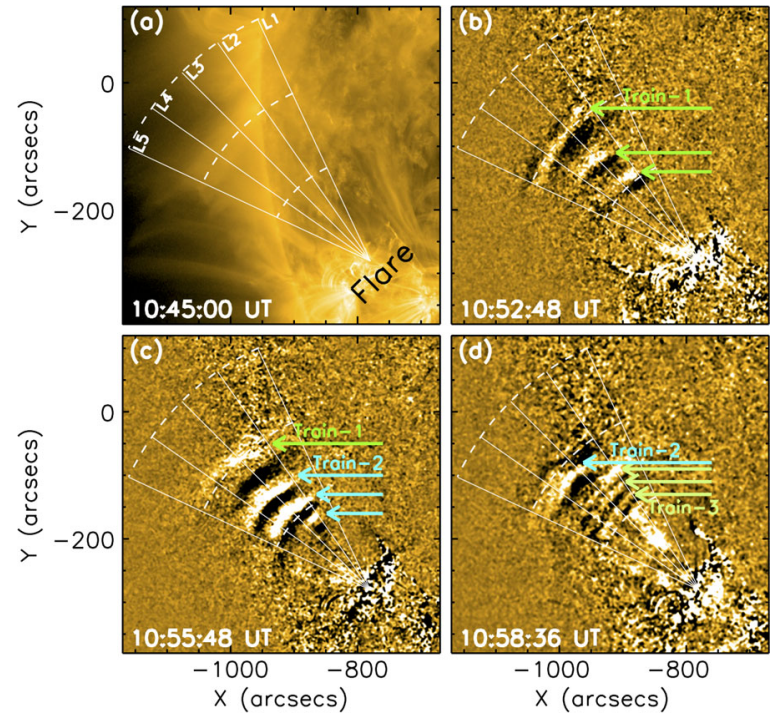

The advanced time resolution and high sensitivity of SDO/AIA led to the direct observations of coronal fast wave trains in the EUV band (Liu et al. 2011). Figure 33 shows snapshots of several fast waves trains of the EUV emission intensity disturbance propagating along the apparent direction of the magnetic field in the corona. The typical speeds of such wave trains are about $1000 \mathrm{~km} \mathrm{~s}^{-1}$, typical periods of the oscillations of the filling signal are about $60 \mathrm{~s}$ and the period varies in time. Typical amplitudes are up to several percent. The waves are detected up to $300 \mathrm{Mm}$ (about half of the solar radius) above the solar surface. Beginnings of individual wave trains coincide with coronal mass ejections or other impulsive energy releases (Yuan et al. 2013).

Ofman et al. (2011) performed the 3D MHD modelling of this phenomena interpreting the oscillations as propagating fast magnetoacoustic waves in a narrow funnel of a dipole field, excited by localised (quasi)-periodic velocity perturbations at the magnetic footpoints of the active region, and found excellent agreement with observations. Pascoe et al. (2013a) developed an advanced numerical model of the dispersive evolution and propagation of fast waves in an expanding magnetic funnel filled in with dense plasma, aiming to interpret coronal EUV fast wave trains. They found that indeed the quasi-periodic fast wave trains are readily formed in plasma funnels. Moreover, the fast wave energy is separated into two wave components - trapped waves which propagate along the funnel axis and leaky components that form similar wave trains outside the funnel. The external wave trains tend to refract upwards. The results were found to be well consistent with observations (Yuan et al. 2013). Moreover, Nisticò et al. (2014) have shown that numerical simulations of rapidly propagating wave trains in an observed active region excellently reproducing the observed behaviour.

\section{Flapping Oscillations of Current Sheets}

One of the common features of coronal and magnetospheric plasma systems is the occurrence of a thin current sheet separating magnetic field lines of opposite orientation. In particular, such current sheets are observed to be extended into the Earth's magnetotail, occur at 
the dayside reconnection site, and are believed to appear in solar flares (see Sect. 11.1) and coronal helmet streamers (Sect. 7.3). MHD waves and oscillations of a current sheet have specific properties. In particular, in-situ measurements in the Earth's magnetotail revealed the low-frequency (about $10^{-4}-10^{-1} \mathrm{~Hz}$ ) magnetic and pressure oscillations of the current sheet (Bauer et al. 1995). One of the most discussed and studied among them are so-called flapping oscillations (see, e.g. Korovinskiy et al. 2015, and references therein), which are also observed in the Jupiter and Saturn magnetospheres. Oscillations (waves) of this type are quasi-periodic displacements of the current sheet in the transverse direction (i.e. along the $z$ axis of the GSM frame of reference in the case of the Earth's magnetotail). Flapping waves are preferentially excited in the central part of the magnetotail at the distances of about 10-30 $R_{\mathrm{E}}$ from the Earth, and propagate towards the flanks (along the $y$-axis of the GSM frame of reference, so along the magnetotail in the direction perpendicular to the direction of the magnetic field) at typical speeds of about $30-70 \mathrm{~km} \mathrm{~s}^{-1}$, which are about ten times lower than the local Alfvén speed. The waves can be directed both parallel and anti-parallel to the electric current of the sheet. They are often observed several minutes prior to the substorm onset and during substorm growth phase. These observational findings give evidence that the flapping waves have roots deep inside the magnetotail and can be internally linked with the substorm activity. The wavelengths and spatial amplitudes of the flapping waves are statistically found to be about $1-5 R_{\mathrm{E}}$ and the characteristic frequency is $\sim 0.035 \mathrm{~Hz}$.

Two different models were developed to explain flapping waves within the MHD framework. The first one interprets the flapping waves as the ballooning-type mode in the curved magnetic field of the current sheet (Golovchanskaya and Maltsev 2005). The physics of this mechanism is ideologically similar to the physics of the internal gravitational waves in the upper atmosphere. The centrifugal force, acting on a plasma element in the curved magnetic field of the magnetotail, plays the role of the gravity. The buoyancy acts on the displaced plasma element as the restoring force. The wavelength range of the ballooning waves predicted by the theory is quite broad - it can be from a fraction of $R_{\mathrm{E}}$ up to the curvature radius of magnetic field lines of the magnetotail, which is estimated in some cases as $\approx 20 R_{\mathrm{E}}$. Thus, in principle, the flapping waves can be interpreted as the ballooning-mode waves. The model predicts both kink-like and sausage-like current sheet displacements. A shortcoming of the theory is that its dispersion relation is derived only for the case when the magnetic curvature radius exceeds the characteristic scale of the flapping waves which is not applicable to all regions of the magnetotail.

The second MHD model of the flapping waves, called the "magnetic double-gradient mechanism", claims that these waves can be caused by the presence of a normal magnetic field component gradient along the current sheet (Erkaev et al. 2007, 2009; Korovinskiy et al. 2015). The characteristic flapping frequency $\left(\omega_{\text {flap }}\right)$, predicted by this model, is determined by the combination of plasma density $\left(n_{\mathrm{e}}\right)$ and two magnetic gradients - the tangential $\left(B_{x}\right)$ and normal $\left(B_{z}\right)$ magnetic field components along the normal $(z)$ and tangential $(x)$ directions of the current sheet respectively:

$$
\omega_{\text {flap }}=\sqrt{\left(\frac{1}{4 \pi n_{\mathrm{e}} m_{\mathrm{p}}} \frac{\partial B_{x}}{\partial z} \frac{\partial B_{z}}{\partial x}\right)_{z=0},}
$$

where $m_{\mathrm{p}}$ is the proton mass. For the Harris-like current density profile Erkaev et al. (2009) have found the analytical expression for the group speed of the flapping waves to be,

$$
V_{\mathrm{gr}}^{\mathrm{kink}, \text { sausage }} \approx \omega_{\mathrm{flap}} \Delta F_{\mathrm{kink}, \text { sausage }}(k \Delta)
$$


where the superscripts kink and sausage denote the kink-like and sausage-like modes, respectively; $\Delta$ is the half-thickness of the current sheet; $k$ is the wave number along the electric current, and $F_{\text {kink,sausage }}(k \Delta)$ are the dimensionless functions:

$$
F_{\text {kink }}(k \Delta)=\frac{1}{2 \sqrt{k \Delta(1+k \Delta)^{3}}}, \quad F_{\text {sausage }}(k \Delta)=\frac{3 k \Delta+4}{2 \sqrt{\left[(k \Delta)^{2}+3(k \Delta)+2\right]^{3}}} .
$$

Substitution of physical parameters typical for the Earth's magnetotail to the above expressions gives values of the frequency and group speed comparable with the parameters of the observed flapping waves. From Eq. (44) it is seen that the stable propagating flapping waves can exist in the case of the positive products of two magnetic gradients, i.e. when the $B_{z}$ component of the magnetic field increases towards the Earth. Stable flapping waves may be excited by some disturbances related with the magnetic reconnection in the magnetotail.

The flapping perturbations become unstable in the case of a negative product between the two magnetic gradients. This physical situation can occur in some places of the current sheet, where the normal magnetic component $\left(B_{z}\right)$ has a local decrease towards the Earth. In particular, this may occur in a place, where the current sheet becomes somehow thinner, and the flapping oscillations grow in amplitude without propagation. The growth time of such kink-like mode instability is of the order of one minute. Stability of a realistic current sheet is provided by the reduction of magnetic field line curvature (Korovinskiy et al. 2013). The Hall effect can also reduce or even totally damp the double-gradient flapping modes of a thin sheet, propagating in the direction opposite to the electric current (Erkaev et al. 2010), but does not affect the waves propagating parallel to the current. The guiding magnetic field (along the $y$-direction) can also stabilise the flapping perturbations (Artemyev and Zimovets 2012; Korovinskiy et al. 2015).

Though flapping waves are a common phenomenon in the Earth's magnetotail, they have not yet been revealed in the solar corona. One of the reasons is that current sheets themselves are not directly identified in the corona yet, although there are a lot of indirect evidences that they should exist there. In particular, the "standard" model of eruptive two-ribbon flares (see Sect. 11.1) predicts formation of a quasi-vertical current sheet above a flaring arcade where magnetic reconnection can take place. Artemyev and Zimovets (2012) considered unstable flapping waves of $2 \mathrm{D}$ quasi-vertical current sheets above a flaring arcade with a sheared magnetic field and suggested that symmetric (sausage-like) flapping waves can modulate the thickness of a current sheet (thus, current density) along the polarity inversion line. As a result, this process can modulate the efficiency of magnetic reconnection and energy release in different parts of a flaring arcade. This mechanism was used to interpret quasiperiodic pulsations of hard X-ray and microwave emissions and motions of its sources along the polarity inversion line, often observed in two-ribbon flares (e.g. Grigis and Benz 2005; Zimovets and Struminsky 2009). In a sheared arcade (with the shear angle $\varphi$ ) the group speed along the current of a possible unstable flapping mode can be estimated as:

$$
V_{\mathrm{gr}} \approx \frac{V_{\mathrm{A}}}{4} \sqrt{\frac{\lambda}{L_{x}} \sin \varphi}
$$

where $V_{\mathrm{A}}$ is the Alfvén speed outside the current sheet, $\lambda$ is the wavelength of the flapping waves, and $L_{x}$ is the scale of the sheet in the vertical direction with respect to the solar surface. Substituting reasonable coronal values to equation (47), $V_{\mathrm{A}}=1000 \mathrm{~km} \mathrm{~s}^{-1}$, $\lambda=1000 \mathrm{~km}, L_{x}=10000 \mathrm{~km}$ (Reid et al. 2011), and $\varphi=45^{\circ}$, results in $V_{\mathrm{gr}} \approx 70 \mathrm{~km} \mathrm{~s}^{-1}$. It is consistent with the observed speeds of the QPPs-source motion along the polarity inversion line (e.g. Grigis and Benz 2005; Zimovets and Struminsky 2009). However, to show 
whether flapping waves are really present in the solar corona or not, additional, more detailed spatially-resolved observations are required. At this moment, it is too early to claim that coronal flapping waves, similar to the magnetospheric ones, do exist.

\section{Sources of MHD Waves}

In the context of the solar corona the mechanisms for the excitation of MHD oscillations are poorly understood. Mainly it is connected with the observational limitations intrinsic to remote sensing. On the other hand, the excitation of MHD waves in the Earth's magnetosphere is reasonably well understood, which creates a solid basis for knowledge transfer.

\subsection{External Generation Mechanisms in the Magnetosphere}

A large group of magnetospheric MHD wave phenomena is excited by mechanisms acting outside the magnetosphere. The driver of these waves is the solar wind: shear flow at the flanks of the magnetosphere, fluctuations, waves, transient events inherent in the solar wind or generated by the interaction of solar wind and the magnetosphere. Such waves of external origin and their penetration through the turbulent magnetosheath to the magnetosphere require a proper physical explanation.

\subsubsection{Shear Flow Instabilities}

The solar wind is a turbulent flow, however, even the almost fluctuation-free steady solar wind flow would be able to drive waves at the magnetopause through shear flow instabilities. Among these, the Kelvin-Helmholtz instability (KHI) is the most widely studied (e.g. $\mathrm{Pu}$ and Kivelson 1983; Walker 2005) and commonly accepted mechanism (see, e.g. Mazur and Chuiko 2015, and references therein, for the recent status of this research). The KHI can develop at a boundary between two counter-streaming MHD media, such as the magnetopause, by amplifying small scale fluctuations into large amplitude waves and vortices, as schematically illustrated in Fig. 34. Speed fluctuations at the perturbed surface cause pressure fluctuations according to Bernoulli's theorem. Where the pressure has a minimum, fluid elements experience a net force opposite to the pressure gradient. In an incompressible fluid this happens where the flow speed is higher than the bulk speed of the medium, i.e. at the largest displacements. The waves grow due to the instability. In the compressible case, density variations can reduce, or in extreme cases even quench the instability (Walker 2005). In a magnetised plasma, the magnetic field component parallel to the relative shear velocity of

Fig. 34 Schematic illustration of Kelvin-Helmholtz instability onset at the magnetosphere flanks. The excited surface modes are coupled with Alfvén field line oscillations

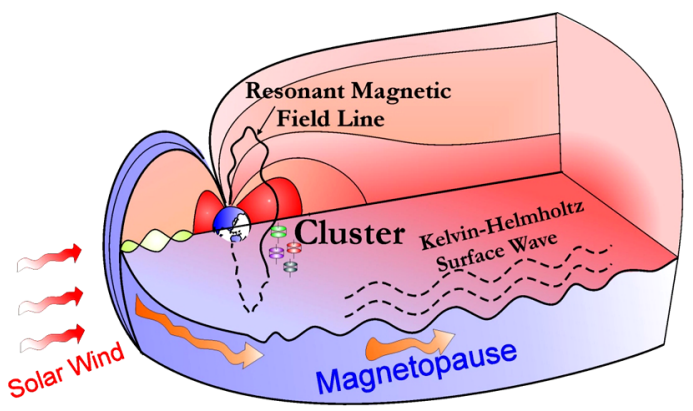


the media also has an important stabilising effect. This is because perturbations due to the instability that acts towards bending field lines are counteracted by the force of magnetic tension.

At the magnetospheric boundary $\mathrm{KH}$ waves manifest themselves in the fluctuations of the plasma (density, velocity, temperature) and magnetic field, typically with a period of the order of minutes. Theory of KHI has developed through several stages since it was first proposed. In the first models of the KHI, the instability was considered an infinitely thin tangential discontinuity separating two incompressible fluids. Pu and Kivelson (1983) were the first to study the KHI in a compressible MHD plasma and model the phenomenon at the terrestrial magnetopause. They identified two compressible modes: the quasi-slow mode and the quasi-fast mode, both coupled to evanescent waves on both sides of the magnetopause. The most important effect introduced by the compressibility is the appearance of a second critical flow speed, above which the instability quenched.

A boundary layer of finite width was first investigated by Lee et al. (1981), and the idea was further developed by Walker (1981) and Miura and Pritchett (1982) by including compressibility. In all cases two surface modes were identified, one at each side of the boundary layer. The highest growing rate was found to be achieved at $k d \approx 1$, where $k$ is the wave number and $d$ is the thickness of the boundary layer. As $V_{\mathrm{ph}}=\omega / k$, it also means that for a given thickness $d$ and phase speed $V_{\mathrm{ph}}$ a quasi-monochromatic KH wave grows.

All of the above theoretical results were achieved based on models valid only in the linear MHD regime. These models can explain how and where the instability may appear, but cannot describe the fully developed, nonlinear phase of the instability. In particular, development of KHI can increase the thickness of the shear flow interface via generation of the effective viscosity (e.g. Mishin 2005). Global MHD models including the nonlinear development of the KHI, based upon full-MHD numerical modelling, appeared almost a decade ago. Claudepierre et al. (2008) was successful in reproducing two KH modes propagating tailward along the outer and inner edges of the low latitude boundary layer (LLBL) for a southward IMF. These two modes were found to occur for $k d=0.5-1.0$ and to have different phase velocities and wavelengths, but oscillating at the same frequency. Larger shear flow velocities were found to excite KH waves of higher frequencies. Merkin et al. (2013) found that the KHI has a 3D nature: the magnetopause is perturbed not only in the equatorial plane, but also in the noon-midnight meridional plane. Surface mode perturbations were found to couple to body modes past the terminator plane. They also found field-aligned currents along closed field lines connecting the shear layer and the ionosphere. Their finding that magnetic and plasma pressures are spatially decorrelated is attributed to nonlinear effects. They identified two regions where the waves grow: one closer to the subsolar point (the point on the Earth where the Sun is perceived to be directly in zenith), and another at the flanks. According to this model, the KHI is initiated around $30^{\circ}$ off the noon meridian (Guo et al. 2010; Li et al. 2012). The second unstable region starts prior to the terminator and lasts to about $-5 R_{\mathrm{E}}$ (meaning that this location is in the anti-sunward direction). The plasma closer the subsolar point is less compressible and hence here the growth rate is higher.

Early observations supporting the KHI origin of high latitude Pc4-5 waves were the reversal of wave polarity at noon and amplitude rapidly decaying with increasing distance from the magnetopause. It was found that these waves propagate away from noon with a phase speed that is independent of frequency. These observations are in agreement with theoretical predictions, i.e. with a tailward propagating surface wave coupled to an evanescent wave in the magnetosphere. In the magnetosphere compressive waves of $\mathrm{KH}$ origin are coupled to local Alfvén field line resonances. Rolled up KH vortices were identified more frequently on the post noon, dusk-flank (Taylor et al. 2012) indicating that the KHI develops 
rapidly into the non-linear phase. The observed $\mathrm{KH}$ wavelengths were found to be in general larger than the wavelength predicted by linear theory (Kivelson and Chen 1995).

Recent multi-spacecraft missions Cluster, THEMIS have offered unique opportunities to observe the KHI in action. Using the observations of the Cluster satellites, Hasegawa et al. (2004) demonstrated the nonlinear growth of KHI and associated vortices along the duskflank during a time period when the IMF was northward. Nishino et al. (2007) observed KHI waves on both flanks simultaneously with Cluster and Geotail spacecraft.

Beside the KHI, the Miles-Phillips instability has also been proposed as a candidate source of surface wave activity on the magnetopause (e.g. Kurazhkovskaya and Klain 2012). The Miles-Phillips instability is also a shear flow instability that is thought to grow waves at any flow speed. Thus for the shear flow speeds lower or higher than the KH critical flow speeds the Miles-Phillips instability could be dominant (Lushnikov 1998). The MilesPhillips instability requires a boundary layer with a continuously changing flow speed. The growth rate is proportional to the curvature of the flow speed profile at a distance $d$ from the boundary, where the flow speed equals to the phase velocity of the surface wave. The prerequisite of both the KH and Miles-Phillips instabilities is the existence of some initial seed fluctuation. Perturbations of any origin can serve as seed fluctuations for these shear flow instabilities. However, the Miles-Phillips mechanism has not yet been investigated in depth for conditions (compressive MHD plasma, finite boundary layer thickness, etc.) typical at the magnetopause.

In the solar corona, development of KHI-driven plasma vortices was observed in the interface between the dimming region and the surrounding corona (Ofman and Thompson 2011), and off-limb at a flank of a rising CME plasmoid (Foullon et al. 2011). In the former study, the vortices were found to range in size from a few to several $\mathrm{Mm}$, and travelled along the interface at the apparent speed of $6-14 \mathrm{~km} \mathrm{~s}^{-1}$. 2.5D numerical MHD modelling supported the interpretation of the observations in terms of KHI. In the latter work, a street of four Alfvénic vortices was seen to develop in about $30 \mathrm{~s}$. It moved along the flank at the speed of about $400 \mathrm{~km} \mathrm{~s}^{-1}$ that was approximately two times lower than the projected speed of the plasmoid. The typical size of a vortex was about $10 \mathrm{Mm}$. The increment was estimated as $0.05-0.03 \mathrm{~s}^{-1}$. The magnetospheric KHI model by Miura and Pritchett (1982) was employed for the interpretation of the observed results.

In the corona, the attention has also been paid to shear-flow instabilities caused by the effect of negative energy waves (Joarder et al. 1997; Holzwarth et al. 2007). In this regime, the presence of a mechanism for draining the energy from magnetoacoustic waves, e.g. dissipation by finite viscosity, thermal conductivity or resistivity, transformation to another mode, or leakage from the system, leads to their amplification. For these instabilities, the threshold value of the flow shear is lower than the KHI threshold. A more detailed study of this phenomenon and its manifestation in the corona and magnetosphere is required.

\subsubsection{Solar Wind Fluctuations}

The solar wind is a turbulent flow, where MHD turbulence and waves of different origins have been identified. The most well-known phenomena are the long-period outward travelling Alfvén waves with around 1 hour period. Waves with shorter periods have been recently identified in the solar wind too, such as electromagnetic ion-cyclotron waves (EMIC, Jian et al. 2009) and kinetic Alfvén waves (KAWs, Podesta 2013).

Solar wind waves with periodicity in the nominal Pc5 band $(1-3 \mathrm{mHz})$ are of special importance for ULF wave physics. Solar wind pressure variations can drive compressive ULF waves that propagate deep into the magnetosphere. This hypothesis is supported by 
both observations and global MHD modelling. One of the sources of high latitude Pc5 pulsations is believed to be this mechanism. The interaction between the magnetosphere and pressure variations with periods higher than $\approx 400 \mathrm{~s}(2.5 \mathrm{mHz})$, i.e. longer than the Alfvénic travel time through the dayside magnetosphere, can be treated as quasi-static. In this case the size of the magnetosphere changes, responding directly to the external pressure variations (Kepko and Spence 2003). The variation of the magnetopause distance is associated with the variation of the magnetopause (Chapman-Ferraro) current. Waves in the magnetosphere are believed to be the magnetic signatures of this pulsating current. Takahashi and Ukhorskiy (2007) called this entry mechanism the breathing mode, namely the induction of magnetospheric ULF pulsations by irregular pressure fluctuations in the solar wind. Another possible, although not well understood, entry mechanism is the direct penetration of solar wind waves into the magnetosphere (see Sect. 13).

Multi-harmonic signals with very stable frequencies (about $1 \mathrm{mHz}, 1.3 \mathrm{mHz}, 2.2 \mathrm{mHz}$, $2.6 \mathrm{mHz}$ ) called the "magic frequencies" are often observed at high latitudes. The magic frequencies were interpreted as supporting evidence for the existence of the magnetospheric cavity mode resonance (see the discussion in Sect. 3.2). Kepko and Spence (2003), however, found the same spectral peaks appearing simultaneously in the solar wind and ground Pc5 pulsations, suggesting that the observed magnetospheric waves were directly driven by the solar wind fluctuations.

ULF wave activity in the solar wind was found to be typically higher in corotating interaction regions (CIRs) between slow and fast solar wind. Hence, the time intervals of the CIRs' arrivals seem to be favourable for the generation of ground ULF waves, typically in the Pc5 band.

Whatever mechanisms cause pressure variations that drive waves in the magnetosphere, they are found to be an important source of terrestrial ULF activity. From the results of a statistical survey based on 10-year solar wind and magnetospheric data (Viall et al. 2009) it was concluded that periodic density structures observed in the solar wind corresponded to $54 \%$ of the discrete frequency peaks detected in the magnetospheric ULF activity.

Solar wind turbulence (see Bruno and Carbone 2013, for a recent review) should also have an important role in driving ULF waves, e.g. by the pressure fluctuations associated with them. The Miles-Phillips mechanism is believed to be responsible for driving small surface perturbations on the magnetopause as a consequence of pressure variations inherent in the solar wind, and seed perturbations can be then amplified by Miles-Phillips or KHI instabilities.

\subsubsection{Waves Generated by the Interaction Between the Solar Wind and the Magnetosphere}

Dayside Compressive Pc3s of the Foreshock Origin ULF waves generated in the upstream foreshock by solar wind protons backscattered from the bow shock are also an important source of terrestrial ULF wave activity. Upstream waves are believed to be the dominant source of dayside Pc3 waves. The mechanism responsible for the foreshock wave activity is a kinetic effect, not described by the MHD Rankine-Hugoniot conditions-the ioncyclotron resonance. Protons reflected from the bow shock travel sunward (at a speed $v_{\mathrm{b}}$ ), gyrating along interplanetary magnetic field lines. Reflected protons generate waves propagating along the same magnetic field lines, as schematically shown in Fig. 35. A resonance takes place when the Doppler shifted frequency of the waves (i.e. in the frame moving with the reflected ions) equals the local proton gyrofrequency: $\omega_{0}-k v_{\mathrm{b}}=-\Omega_{\mathrm{i}}$, where $\omega_{0}$ is the wave frequency in the solar wind plasma frame, and $k$ is the wave vector. The generated 


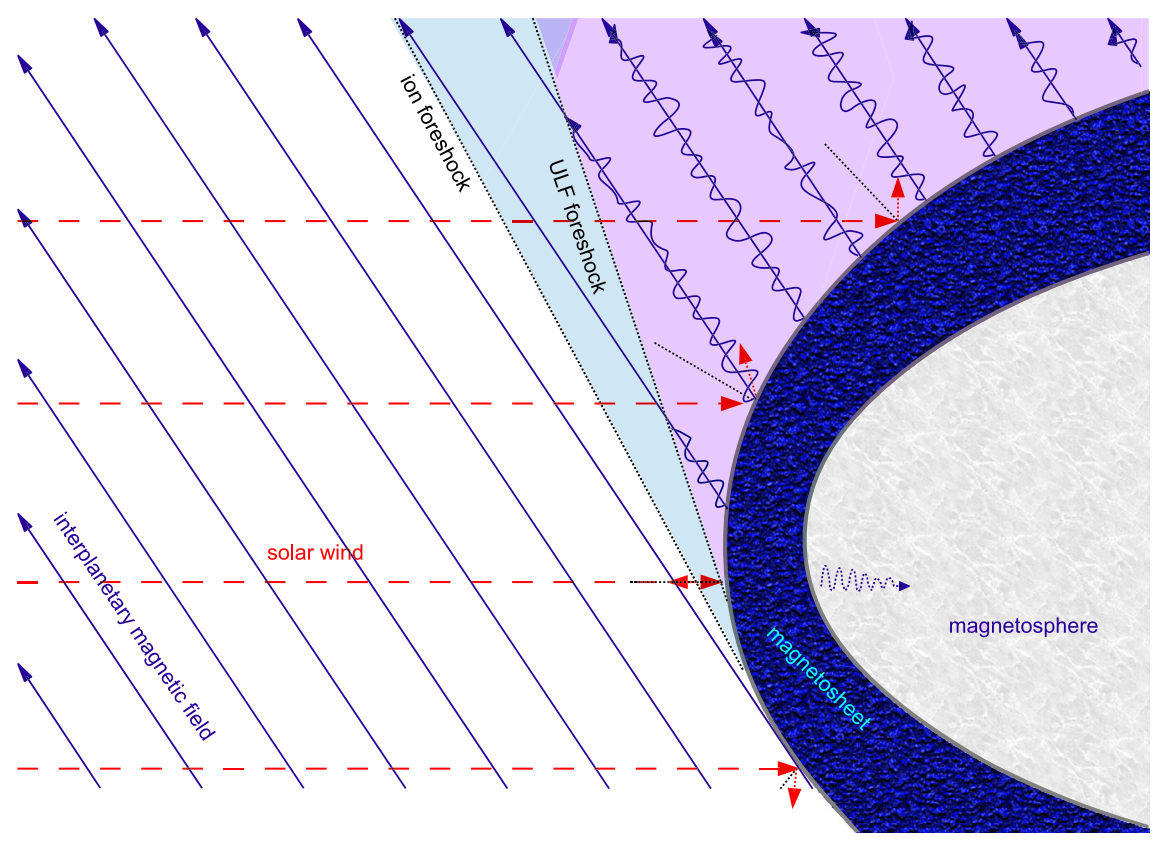

Fig. 35 Sketch of upstream waves generated in the quasi-parallel foreshock region by solar wind protons backscattered from the Earth's bow shock

waves travel sunward along the magnetic field lines at the Alfvén speed. However, since the solar wind is typically super-Alfvénic, upstream waves are swept back towards the bow shock too. Here they may enter the magnetopause and propagate deep into the magnetosphere as fast magnetoacoustic waves (Heilig et al. 2007). Due to their cyclotron origin, the frequency of upstream waves depends on the interplanetary magnetic field strength. Their amplitude as observed on the ground is determined by solar wind parameters, e.g. the IMF direction, solar wind speed and density.

Upstream waves have been observed on the ground and also in the near Earth space for decades (see Troitskaya 1994, for a review). Moreover, they have been identified at other planets, as well. The role of the solar wind plasma density, however, was recognised only recently (Heilig et al. 2010). The density affects upstream wave generation, the amount of Doppler shift and the efficiency of reflection from the bow shock through the densitydependent variation of the Alfvénic Mach number, as well as the size of the magnetosphere the waves have to travel through to reach the ground. All of the above dependencies can be explained, at least qualitatively, by the ion-cyclotron model. The main entry channel of upstream waves was found at the subsolar point of the magnetopause, however, it is likely that upstream waves can enter the magnetosphere also in the dayside cusp region.

Magnetopause Surface Modes There is a growing evidence that surface mode resonances on the magnetopause standing between the conjugate ionospheres (coupled to the evanescent mode in the magnetosphere) can also develop. In the so-called KruskalSchwarzschild mode the magnetopause behaves like a membrane resonantly interacting with the magnetospheric cavity or waveguide modes. The "magic frequencies" may be interpreted as the manifestation of this surface's eigenmodes (Sect. 3.2). An example of such an 
event was observed by three THEMIS spacecraft reported by Plaschke et al. (2013). They argued that the standing mode was not generated by KHI but by some compressive fluctuation generated in the upstream or quasi parallel foreshock.

Pressure Pulses and Impulsive Excitation Not only regular, wave-like pressure variations, but also single pressure pulses drive waves in the magnetosphere. Pressure pulses at the magnetopause excite compressive waves that can propagate isotropically anti-sunward. In the highly inhomogeneous plasma of the magnetosphere these incoming compressive waves can drive compressive cavity modes and couple to shear Alfvén field line resonances. Major pressure pulses are usually linked to the arrival of CMEs or CIRs. Pressure pulses compress the whole magnetosphere. The associated jump in the Chapman-Ferraro current strength manifests itself on the ground as a sudden change in the geomagnetic north component. This impulsive magnetic signal if followed by the activation of the Dst index (ring current) is called a storm sudden commencement (SSC), if not, it is called a sudden impulse (SI).

Specific transient Psc pulsations have been observed from high to low latitudes right after SSCs or SIs. Long-period damped pulsations with several spectral peaks are typically observed on the ground and also in the magnetosphere. On the ground, at magneticallyconjugate stations, oscillations in the north magnetic field component, $H$, appear in-phase, while the signal in the east component of the magnetic field $D$ is out-of-phase, evidencing the excitation of the fundamental Alfvén mode. Psc pulsations are generally interpreted as the complex resonant response of the magnetosphere to a pressure pulse: compressive mode cavity/virtual resonances (e.g. Yumoto et al. 1997; Fujita et al. 2000) or magnetopause surface mode oscillations, often accompanied by local field line resonances. Virtual resonance is a unique type of radial (i.e. across the magnetic field, thus fast magnetoacoustic) standing wave which may develop even in a case when the plasmapause is not well defined, i.e. it is not a perfect reflective boundary. The effect of virtual resonance was first found by theorists using a quantum mechanics approach as a solution of the MHD wave equations in a radially stratified plasma (Lee and Kim 1999). The nature of this resonance can be illustrated considering a one-dimensional non-uniformity of the fast speed (or the Alfvén speed in the low- $\beta$ case) across the field. If there is a "well" in the profile of the speed, there can exist trapped fast magnetoacoustic waves, with the oscillatory transverse structure in the well, and evanescent outside it. Thus, in this case the transverse profile of the speed constitutes a cavity for the fast waves. This situation, e.g. corresponds to the radial structure of the magnetosphere with the ionosphere and the plasmapause, and, in the coronal context, to the fast waves trapped in a coronal loop (see Sect. 4). However, if in one side of the cavity the fast speed has a crest (a localised maximum) but then decreases again, the wave trapped in the cavity can tunnel through the potential barrier formed by the crest, and become oscillatory again. This lead to the leakage of the wave from the cavity. The real part of its frequency is prescribed by the parameters of the cavity, and may have a set of well-pronounced peaks at the resonant frequencies. This is the effect of virtual resonance.

In particular, the virtual resonance may significantly modify the spatial structure of the fast magnetoacoustic mode of the cavity, e.g. leading to the appearance of a maximum of the perturbation at the cavity boundary, rather than a node. Linear MHD simulations in a dipole field geometry could successfully reproduce the generation of broadband compressive waves by pressure pulses and their coupling to shear Alfvén modes throughout the magnetosphere (Lysak and Lee 1992). Lee and Kim (1999) identified compressive standing waves in the inner magnetosphere and interpreted them as the plasmaspheric virtual mode resonance.

A very similar mathematical approach has been applied to the theoretical description of vertically-polarised oscillations in coronal loops (Brady et al. 2006). The transverse (across 
the magnetic field directed along the loop) structure of the perturbations is determined by the effective potential well formed in the loop by the transverse non-uniformity of the fast magnetoacoustic speed. However, higher up, the speed decreases, forming an effective potential crest. The effective potential well leads to the formation of the discrete spectrum of the oscillations that tunnel through the crest and leak upwards in the region where the fast speed is lower than inside the effective potential well. Similar mathematics describes the appearance of the observed discrete spectrum of 3-min oscillations in sunspot umbral chromospheres (Botha et al. 2011). In this case, the virtual acoustic resonator is formed by a field-aligned non-uniformity of the plasma density and temperature.

Investigating the global current systems driven by pressure pulses at the magnetopause by numerical simulation, Fujita and Tanaka (2006) found that compressive waves launched by the pulse and propagating in the magnetosphere have a crucial role in storm-time dynamical processes. Behind the wave front the kinetic energy of the plasma is converted into electromagnetic energy, driving currents that connect the field-aligned currents associated with the preliminary impulse and the main impulse.

Plasma Transfer Events (Impulsive Penetration) The solar wind flow and interplanetary magnetic field are not stationary on time scales corresponding to the time needed for the solar wind to encompass the magnetosphere, and not uniform on spatial scales smaller than the size of the magnetosphere. As a consequence localised plasma irregularities (also called plasmoids) at the bow shock may occur. These diamagnetic plasmoids impact the magnetopause (like rain drops on the surface of the water) shaking it continuously (Lemaire 1985; Echim and Lemaire 2000). Under some circumstances a plasmoid of solar wind origin can be injected into the magnetosphere. Once inside the magnetosphere it expands along field lines. This process is termed impulsive penetration. It is also known as a plasma transfer event (Woch and Lundin 1991). The plasmoid moving in the Earth-magnetic field acts as a dynamo driving transient field-aligned current, which may produce a ULF wave bursty response.

Flux Transfer Events Flux transfer events (FTEs) were first realised from their magnetic signature by Russell and Elphic (1979), however, such signatures can also be due to plasma transfer from the solar wind into the magnetosphere. FTEs are patchy (spatially and temporally limited) reconnections at the magnetopause. They form a magnetic portal as large as the size of the Earth through which the magnetosheath and magnetospheric plasma can flow. They tend to open at low latitudes at the southward IMF, then gradually roll over the winter pole (i.e. the pole in the winter hemisphere of the Earth). Damped Pc5 pulsations associated with FTEs (Fukunishi and Lanzerotti 1989) are believed to be driven by the convection of magnetic islands along adjacent closed field lines (dynamo-mechanism) (Lee et al. 1988).

There are still a number of open questions connected with the external mechanisms for the excitation of magnetospheric MHD waves. Some of them are as follows:

- It is necessary to elucidate the relative roles of different external generation mechanisms of the ULF waves with the large azimuthal wave lengths; that is, we need to find out the nature of the main wave channel of the solar wind energy penetration into the magnetosphere.

- Usually, azimuthally large-scale low- $m$ Alfvén waves are thought to be generated by the external sources. Is it possible that a part of them are generated or triggered by internal sources, including unsteady currents inside the magnetosphere or ionosphere? Would it be possible to distinguish those internally-generated waves from externally generated low- $m$ pulsations? 


\subsection{Internal Generation Mechanisms}

A physical substantiation for the categorisation of Alfvén waves into the azimuthally largescale and azimuthally small-scale modes is the difference of the driving source. The generation mechanism for the low- $m$ modes is supposed to be provided by the field line resonance phenomenon: a fast magnetoacoustic wave is generated on the magnetopause and propagates into the magnetosphere generating a shear Alfvén mode on a magnetic surface where the wave frequency $\omega$ equals the local Alfvén frequency. However, this mechanism is not effective for the high- $m$ Alfvén waves, since in this case the fast magnetoacoustic mode transparent region is very narrow and situated just in the vicinity of the magnetopause, and only an exponentially small part of the wave energy is able to penetrate deep into the magnetosphere. Hence, some inner source of energy must be found for the high- $m$ waves. In fact, poloidal Alfvén waves can effectively interact with energetic particles drifting in the magnetosphere in the azimuthal direction by means of the wave's azimuthal electric field. For this reason these particles are considered as a natural energy source of the high- $m$ Alfvén waves.

\subsubsection{Kinetic Alfvénic Instabilities}

Waves can exchange energy with the particles by means of the transverse electric field $e_{\perp}$, the parallel electric field $e_{\|}$, and parallel magnetic field $b_{\|}$. In the case of ULF waves, when $\omega \ll \Omega_{\mathrm{i}}$, the temporal variation of the particle energy is described by the expression

$$
\frac{\partial \varepsilon}{\partial t}=q \mathbf{v}_{\|} \cdot \mathbf{e}_{\|}+q \mathbf{u}_{d} \cdot \mathbf{e}_{\perp}+\mu \frac{\partial b_{\|}}{\partial t},
$$

where $q$ is the electric charge, $\varepsilon=m v^{2} / 2$ is the kinetic energy and $\mu=m v_{\perp}^{2} / 2 B$ is the magnetic moment, $\mathbf{u}_{d}$ is the drift velocity in the inhomogeneous magnetic field. In the MHD approximation the parallel electric field is small. The effective exchange of energy between ULF waves and particles can occur under the drift-bounce resonance condition

$$
\omega-m \bar{\omega}_{\mathrm{d}}-K \omega_{\mathrm{b}}=0,
$$

where $\bar{\omega}_{\mathrm{d}}$ is the bounce averaged angular drift velocity, $K$ is an integer called the bounce harmonic number, and $\omega_{\mathrm{b}}$ is the proton bounce frequency. The special case $K=0$ is usually called the drift resonance. As resonant particles, the substorm injected protons with energies $\varepsilon=10-150 \mathrm{keV}$ are usually considered. Note that the high- $m$ oscillations usually (albeit not always) propagate westward, in the direction of the positive ion drift. Due to symmetry properties, the drift resonance can generate the odd harmonics (including the fundamental harmonic $N=1$ ), while the drift-bounce resonance with $K= \pm 1$ can generate the even harmonics (e.g., $N=2$ ).

Whether the wave-particle interaction will lead to the transfer of energy from the wave to the particles (i.e., collisionless damping of the wave) or in the reverse direction (instability) depends on the particle distribution function $F$. The expression for the instability growth rate $\gamma$ can be written in the form

$$
\gamma \propto \operatorname{Im} \sum_{K=-\infty}^{\infty}\left\langle\frac{\omega-\omega_{*}}{\omega-m \bar{\omega}_{d}-K \omega_{\mathrm{b}}} \frac{\partial F}{\partial \varepsilon}\right\rangle,
$$

where $\langle\ldots\rangle$ means averaging over the velocity space, and $\omega_{*}$ is the diamagnetic drift frequency proportional to the radial gradient of the equilibrium distribution function. 
Fig. 36 Poloidal Alfvén waves in the duskside magnetosphere, detected by three THEMIS probes as oscillations of the magnetospheric magnetic field magnitude

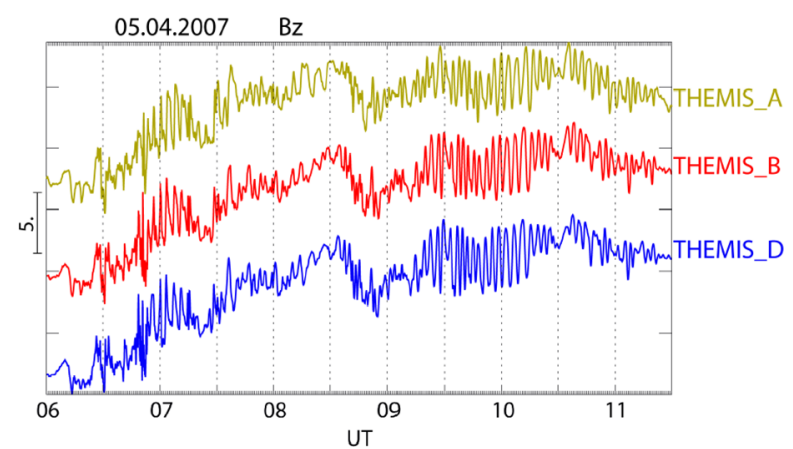

In a plasma with monotonous energy distribution, $\frac{\partial F}{\partial \varepsilon}<0$ for all energies (e.g. the Maxwellian distribution), and homogeneous density/temperature, $\omega_{*} \approx 0$, the growth rate is negative, $\gamma<0$ (see Eq. (50)). Thus, the wave experiences collisionless damping. However, there are two possible sources of free energy that can cause instability and hence the wave amplification:

- A non-monotonic energy distribution of energetic particles, i.e., when the distribution function has the positive slope at some energies, $\partial F / \partial \varepsilon>0$. The distributions of this kind are usually called the inverted, or bump-on-tail distributions.

- A plasma can be unstable in presence of large spatial gradients of the particle number density or mean particle energy, when $\omega<\omega_{*}$.

Direct estimates of azimuthal wave numbers $m$ and azimuthal phase speeds $\omega / m$ became possible thanks to ionospheric radar measurements and magnetospheric multi-satellite observations. Typical azimuthal phase speeds of compressive storm-time Pc5 pulsations coincide with the drift velocities of energetic, 10-40 keV ions, which can be considered as a signature of the drift resonance. In the example shown in Fig. 36, three THEMIS probes moved in a pearl-on-string configuration in the equatorial plane in the dusk sector, with small separation in the longitude $\Delta \Lambda<2^{\circ}$. A large value of the compressive component $b_{\|}$is typical for poloidal Alfvén oscillations in a plasma with $\beta \simeq 1$. This observational configuration enabled the determination of the phase delay between the signals measured by different satellites, which corresponded to $m \simeq 30$. Such high $m$ and consequently small phase speeds are typical for storm-time Pc5 pulsations excited by energetic protons during the recovery phase of magnetic storms, when the density of energetic particles is still high. However, it is still not clear whether the proton fluxes can remain high enough in this region four days after the main phase after a storm.

A distribution function with the bumps ("inverted distribution function") can naturally appear in the magnetosphere in two ways. First, the dispersion of the energies of the substorm injected particles leads to the dispersion of their drift velocities, and particles with the larger energies reach a particular location on the azimuthal coordinate earlier than low energy particles. The inverted distribution can also appear under steady state conditions due to the existence of a global magnetospheric electric field causing the energy independent $\mathbf{E} \times \mathbf{B}$ drift (Cowley and Ashour-Abdalla 1976).

Though this instability is a viable mechanism for high- $m$ pulsation generation, and has received considerable attention to date, it would not be fair to shut our eyes to several inherent problems of this theory:

- In order to understand the spatial structure of the mode generated by the instability it is necessary to solve the general problem that takes into account both plasma inhomogeneity 
and the resonant particles. Klimushkin (2000) considered the effect of kinetic instabilities on the spatial structure of monochromatic Alfvén waves. A high- $m$ Alfvén wave propagates across magnetic shells from the poloidal to toroidal surfaces, changing its polarisation from poloidal to toroidal. Simultaneously the wave interacts with energetic particles and acquires some of their energy. The instability growth rate is maximal on the poloidal surface and decreases to nearly zero on the toroidal surface. Thus, the maximum of the wave amplitude must take place when the wave has toroidal polarisation. The only factor which can prevent the wave transformation is wave damping due to the finite ionospheric conductivity. If the damping is stronger than the instability, the wave will be attenuated near the poloidal surface keeping poloidal polarisation. Thus, it is the ionospheric damping rather than the instability which is favourable to poloidal polarisation (Klimushkin 2007).

- Instability theory has some difficulties with the determination of the azimuthal wave number $m$ of the generated wave. Usually, the $m$ value is inferred from the resonance condition (49),

$$
m=\frac{\omega-K \omega_{\mathrm{b}}\left(\varepsilon_{\mathrm{res}}\right)}{\bar{\omega}_{d}\left(\varepsilon_{\mathrm{res}}\right)},
$$

where the bounce and drift frequencies are both calculated for the resonant energy $\varepsilon_{\text {res }}$, determined from the observed inverted distribution function. Although such a calculation usually shows a good agreement with the measured $m$ number (Wright et al. 2001; Baddeley et al. 2002), it is only a part of the story. To determine the expected $m$ number of the wave generated by the kinetic instability, it is necessary to perform an integration over space and energy and to find the growth rate $\gamma(m)$. This calculation was performed by Mager and Klimushkin (2005), showing that the principal harmonic waves $(N=1)$ can be generated by the drift resonances, $K=0$. In this case, the waves in a rather narrow range of the $m$ numbers can be generated. If the bump-on-tail is centred on the energy $10 \mathrm{keV}$, then $m=-50 \pm 10$ ( $m<0$ means the westward phase motion), and $m=-15 \pm 5$ for the energy $150 \mathrm{keV}$. However, for the second harmonic waves $(N=2)$ generated by the driftbounce resonances with $K=+1$ the range of the $m$ numbers is very wide: $m=-50 \pm 50$. As we see, for the most widely observed second harmonics, the drift-bounce instability does not select any definite value of an azimuthal wave number $m$. This raises the question: if the second-harmonic poloidal waves are generated by the bump-on-tail instability, what determines their actual azimuthal wave numbers?

\subsubsection{Moving Sources}

High- $m$ waves could also be generated by a non-kinetic mechanism that involves their excitation by azimuthally drifting proton clouds injected during substorms. This effect is similar to the Cherenkov emission or Kelvin ship waves on the water surface. The cloud of particles injected into the axially-symmetric magnetosphere at some instant of time $t=0$ can be viewed as a sudden pulse propagating from one azimuthal location to another, at the drift angular velocity $\omega_{\mathrm{d}}(x)$. Each field line oscillates with its own eigenfrequency $\omega_{\mathrm{A}}(x)$. Hence, the wave phase is determined by the expression

$$
S=-\omega_{\mathrm{A}}(x) t+m \varphi,
$$

where $\varphi$ is the azimuthal angle. For the azimuthally drifting source, the $m$ number is determined by the generation mechanism. In the reference frame of the source, the phase just 
behind the source must be constant, thus the Doppler-shifted wave frequency $\omega-m \omega_{\mathrm{d}}$ must be zero. Hence, we obtain the expression for the $m$ number,

$$
m(x)=\frac{\omega_{\mathrm{A}}(x)}{\omega_{\mathrm{d}}(x)} .
$$

Hence, Eq. (52) for the phase is replaced by

$$
S=-\omega(x)\left[t-t_{0}(\varphi, x)\right]
$$

where $t_{0}=\varphi / \omega_{\mathrm{d}}(x)$ is the time when the source passes through the point with the given azimuthal coordinate. The wave electric field is determined as

$$
E(t)=\Theta\left(t-t_{0}\right)|E| e^{i S},
$$

where the Heaviside step function $\Theta\left(t-t_{0}\right)$ indicates that the wave appears in some azimuthal location simultaneously with the particle cloud arrival in the same spot, as the Alfvén wave does not transfer energy across the field lines: the wave can be viewed as to be carried by the cloud.

The wave frequency is fully determined by the radial coordinate and does not depend on the nature of the source. The azimuthal wave number depends on the particle energy because it is defined by the condition (53) and the drift frequency depends on the particle energy $\varepsilon$ as $\omega_{\mathrm{d}} \simeq 8 \times 10^{-3} \varepsilon L$. Thus, one can expect different $|m|$ values for the wave generated by electrons and ions. Its sign is determined by the direction of the drift. It points westward (eastward) if the wave is excited by ions (electrons). This behaviour agrees well with satellite data.

The moving source theory also provides some insight into the wave field evolution with time. The time dependence of the radial wave vector is determined by

$$
k_{x}=\frac{\partial S}{\partial x}=-\left(\frac{\omega}{\omega_{\mathrm{d}}}\right)^{\prime}\left(\omega_{\mathrm{d}} t-\varphi\right)-\omega t \frac{\omega_{\mathrm{d}}^{\prime}}{\omega_{\mathrm{d}}} .
$$

Its sign depends on the drift velocity profile and varies with space and time. Typically, the drift frequency increases with the radial coordinate. Since in most of the magnetosphere the Alfvén eigenfrequency decreases with $L$, i.e. $d \omega / d x<0$, then the first right-hand side term of Eq. (56) is positive when $t>t_{0}$ and grows with time, but the second one is always negative. Thus, there is a special time instant $t_{1}=\varphi \frac{\left(\omega / \omega_{\mathrm{d}}\right)^{\prime}}{\omega^{\prime}}$ when $k_{x}=0$. At the time $t_{1}$ the Alfvén wave becomes purely poloidal, $B_{y}=0$. However, the wave polarisation changes with time. Before and after $t_{1}$ its polarisation is mixed between the poloidal and toroidal. The behaviour of the polarisation ellipse of the wave, predicted by this theory (Fig. 37) is consistent with observational findings of Zolotukhina et al. (2008).

This moving source theory gave a simple explanation of the peculiar feature of the high- $m$ pulsations observed with radars: the equatorward phase propagation (e.g. Fenrich and Samson 1997; Yeoman et al. 2006). Indeed, when $t_{0}<t<t_{1}$, the radial component of the wave vector is negative, $k_{x}<0$, which corresponds to the equatorward phase propagation when projected onto the ionosphere along the field lines.

There are plenty of open questions connected with the internal mechanisms for the excitation of magnetospheric MHD waves:

- We still do not have a realistic theory of the spatial structure and temporal evolution of the particle-driven ULF waves in the magnetosphere. 
Fig. 37 Polarisation ellipses of the wave excited by the moving cloud at different time instants. Dashed lines represent the drifting source. The bold line corresponds to $k_{x}=0$ (purely poloidal polarisation of the wave). The right and left parts of the figure correspond to electrons and ions, respectively. Thin lines represent the lines of the constant phase. From Zolotukhina et al. (2008)

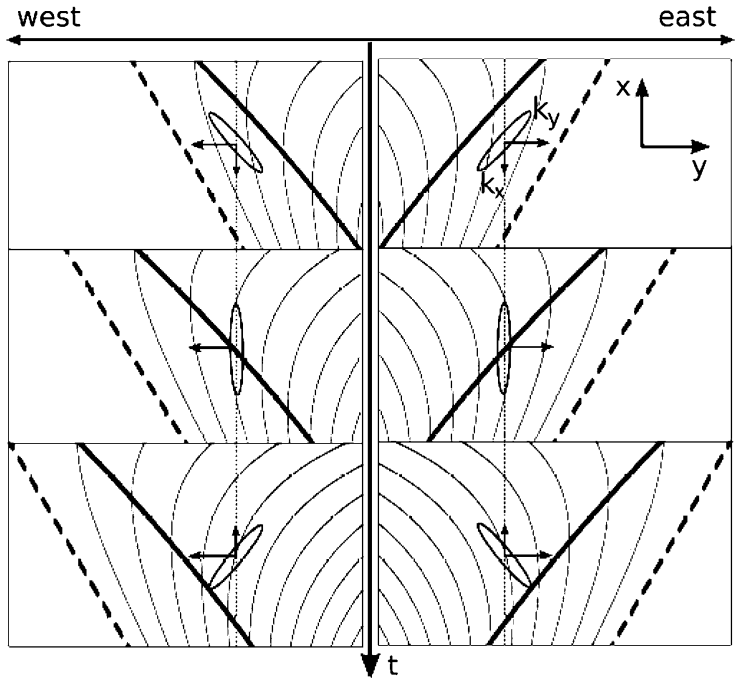

- The physical nature and generation mechanisms for the compressional Pc5 waves in the magnetosphere are not well understood to date. Are they just Alfvén waves in a finite-pressure plasma, or a kind of the compressional waves of kinetic origin, like driftcompressive or drift-mirror modes?

- It is possible that a moving source (e.g., substorm-injected particles) generates an "embryo" wave, which then is amplified by some kind of kinetic instability, possibly via interaction with the same particles. However, theory of this process is still lacking.

- Possible ionospheric mechanisms for the high- $m$ waves' generation have almost not been studied to date.

- It is not clear whether any MHD instabilities (e.g. ballooning instability) could play any role in the generation of high- $m$ waves in the magnetosphere. A theory of this instability has not been developed so far to a level that allowed its direct comparison with the observational data.

- Probably ULF waves with small azimuthal wavelengths play an important role in energisation of the charged particles in the magnetosphere. This role must be theoretically evaluated, and characteristic signatures of this energisation should be found.

- There are some observational signatures that high- $m$ waves play an important role in the substorm development (Rae et al. 2014). This scenario is possible when these waves are generated by some internal source (e.g., kinetic instability) and this way provides a condition for the onset of a large-scale MHD instability. The latter eventually results in the substorm onset. However, links of this chain should be understood yet.

\subsection{Excitation of Kink Oscillations of Solar Coronal Loops}

Understanding the mechanism for the excitation of MHD waves is one of the main current goals in coronal physics. The generation and the consequent evolution of MHD modes do not only depend upon the properties of the magnetic waveguide (density stratification, geometrical structure, multi-stranded internal structure, etc.), but also on the characteristics of the driver (e.g. impulsiveness, spatial location, spatial broadness). Impulsive energy releases, such as flares and CMEs, are usually regarded as drivers for the excitation of fast and slow MHD waves (see Sects. 2, 6 and 7), while details of the excitation mechanisms remain 
Fig. 38 Sketch of the excitation mechanism for horizontally polarised kink oscillations of coronal loops by a steady up flow, based upon periodic shedding of Alfvénic vortices

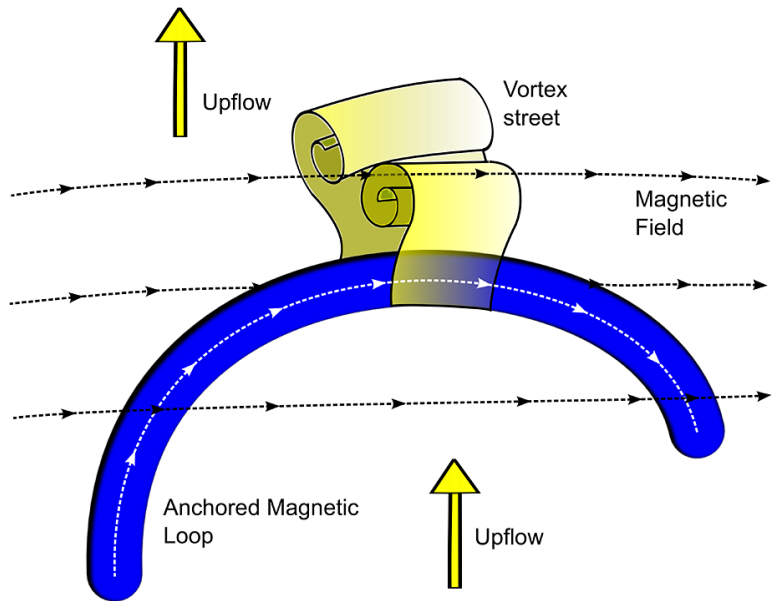

unknown. Here, we briefly describe the state-of-the-art of mechanisms for the excitation of kink modes in coronal loops.

The first evidence of kink oscillations with TRACE (Aschwanden et al. 1999; Nakariakov et al. 1999) in association with a flare led to the conclusion that a flare-generated blast wave may be responsible for the excitation of transverse displacements of a coronal loop: the loop is pushed outward by the flare-generated shock wave and then freely oscillates with the period prescribed by the density contrast $\rho_{0} / \rho_{\mathrm{e}}$ inside and outside the loop, and its length-to-width ratio $L / a$. In this scenario, horizontally and vertically polarised oscillations are not necessarily excited with similar efficiency, because of the effect of the location of the loop within the set of field lines having the same magnetic connectivity (Selwa et al. 2011). This result is consistent with observations, as vertical kink oscillations (e.g. Wang and Solanki 2004; White et al. 2012; Kim et al. 2014) have been detected much more rarely than horizontal. However, this scenario is not fully confirmed by numerical simulations, which show that it is difficult to excite perturbations with the observed displacement amplitude in a loop with a low $\rho_{0} / \rho_{\mathrm{e}}$, e.g. of 2 or 3 , typical for EUV coronal loops (e.g. Terradas et al. 2007; McLaughlin and Ofman 2008; Ofman 2009a). In addition, it is easy to find events where kink oscillations are not triggered by a strong flare when no CME is accompanied.

The preferential excitation of horizontal oscillations of coronal loops may be explained in terms of Alfvénic vortex shedding due to a plasma upflows associated with the lift-off of a coronal mass ejections or eruptions. Indeed, the loop top is regarded as an obstacle or bluff body that modifies the flow pattern and generates a sequence of vortices shed alternately from either side of the loop, forming a downstream von Kármán vortex street. If the magnetic field in the plasma that is dragged in the vertical direction by the flow is parallel to the loop, the loop top experiences a periodic force which is perpendicular to both the flow velocity and the axis of the loop, and hence is in the horizontal direction, see Fig. 38. The period of the force is determined by the flow speed $v$ and the loop's minor radius, $a$, as

$$
P_{\mathrm{vort}} \approx 10 a / v
$$

The oscillations are most effectively excited when the force caused by vortex shedding is in resonance with the natural frequency of the kink oscillations, e.g. with the frequency of the global mode, $P_{\text {vort }} \approx P_{\text {kink }}$ (see Eq. (41)). This model could explain the selectivity in the excitation of the loops oscillations (only those whose natural kink frequency matches the 




Fig. 39 Schematic illustration of the most common mechanism for the excitation of the high-amplitude decaying kink oscillations of coronal loops. (a) Pre-eruption state of the solar active region. (b) Displacement of a coronal loop (solid black curve) from its equilibrium position (dashed black line) by an erupting and expanding plasma structure (low-coronal eruption, LCE), e.g. a flux rope (grey loop-shaped structure). (c) Oscillatory relaxation of the loop to its equilibrium state after the eruption. From Zimovets and Nakariakov (2015)

vortex shedding frequency are excited) and the initial growth of the oscillation amplitude, detected in some cases (Nakariakov et al. 2009; Gruszecki et al. 2010). However, observational confirmation of this mechanism is still absent.

Oscillating loops detected close to topologically unstable magnetic regions, i.e. near the magnetic separatrix, led to the idea of excitation by rocking motions at the photospheric level. In this model, a small displacement of the loop footpoint caused, e.g., by a wave propagating in the photosphere, is magnified by the sensitivity of the equilibrium magnetic topology to a small perturbation (Schrijver and Brown 2000; White et al. 2013). Thus, in this model the observed large-amplitude displacements of the loop in the corona are not connected with any natural mode of oscillation, but are simply adiabatic gradual occurrence of a series of equilibria. This model is not commonly accepted, as the observed periodicities are close to the expected natural frequencies of kink oscillations.

Furthermore, the evidence of the new regime of low-amplitude decay-less kink oscillations against the standard rapidly-decaying regime (Sect. 6.1) raises new questions about the mechanism of excitations. These transverse oscillations of a new kind are not seen to be associated with flares or any bursty energy releases. The constant amplitude may result from some balance between persistent external driving, e.g. by granulation motion and leakage of sunspot oscillations, and damping, e.g. by resonant absorption.

Recently, Zimovets and Nakariakov (2015) have performed detailed statistical study of a large sample of kink oscillation events in the corona observed in the EUV band by the AIA/SDO in 2010-2014. Special attention was payed to the high-amplitude rapidly decaying kink oscillations of coronal loops. The decay-less kink oscillations were not studied in that work. Almost all events studied were found to be accompanied by flares and lower coronal eruptions/ejections (LCEs for short). Thus, both these phenomena of solar activity could equiprobably be the cause of excitation of the oscillations. However, careful analysis of the observational data revealed that it is the LCEs which do excite the loop oscillations, but not the blast waves from flares. It is schematically illustrated in Fig. 39, c.f. also Fig. 7. Before the oscillation event, coronal loops are in their equilibrium state (Fig. 39a). During the event, an erupting plasma structure - a magnetic flux rope or a system of unstable loops (i.e. an LCE) - interacts with some (but not necessarily all) loops by its ram and/or magnetic pressure, causing the loops to displace from their equilibrium positions (Fig. 39b). After the erupting object has left the interaction region, the disturbed loops relax to the pre-eruption or to a new equilibrium state (Fig. 39c). Because of the inertia, the loops overshoot the equi- 
librium, and the decaying kink oscillations occur. Most events (95\%) were established to follow the same scenario. Probably, the efficiency of this mechanism depends on the interaction time of the LCEs with loops and on relative angles between their axes. This should be checked in further researches. The found scenario is consistent with the previously observed domination of the horizontal polarisation of the kink oscillations of loops. However, it should be noted that in $5 \%$ of the studied events it was not possible to unambiguously determine the cause of the oscillations because of limitations of the observational data. It is not excluded that kink oscillations in those events could be excited by some other mechanisms than the discussed above.

Concerning the excitation of propagating kink waves (Sect. 7.2), observational works (e.g. Tomczyk and McIntosh 2009) showed that the waves have a power spectrum in which a power bump at the p-mode frequencies, about 5 minutes, is observed. Actually, the pmodes that are acoustic oscillations of the solar interior do not usually propagate in the corona, as they are evanescent above the photosphere. However, in the presence of magnetic structuring of the plasma, they can perhaps leak upwards and be somehow converted in kink waves. In particular, De Pontieu et al. (2005) proposed that p-modes could reach coronal heights by nonlinear steepening of field-aligned acoustic waves in the case when the field is inclined away from the vertical. More realistic models for such a transfer of p-mode energy in the corona are needed to assess this possibility.

\section{Interaction of MHD Waves with Partly-Ionised Boundaries}

The ionosphere is the geophysical shell where the energy transfer from the Earth's environment to the atmosphere occurs. Therefore, the description of processes in this region is of key importance for the solar-terrestrial physics, and it is particularly important for the MHD wave interaction with the ionosphere. ULF variations of the terrestrial electromagnetic field are effective tools for a continuous monitoring of dynamical phenomena in the magnetosphere and ionosphere. To apply effectively the ground-based magnetometer data it is important to know how well the ground distribution of the ULF field reflects the relevant wave structure in the magnetosphere.

In the context of the solar corona, the effects of partial ionisation come into play at the footpoints of coronal plasma structures, rooted at the dense and partly-ionised chromosphere. Also, a partly-ionised plasma can be found in solar prominences and cool jets.

\subsection{Interaction of Long-Period MHD Waves with the Thin Ionosphere}

The analytical solution to the problem of the Alfvén wave interaction with the coupled magnetosphere-ionosphere-atmosphere-ground system can be found under the "thin ionosphere" approximation. This approximation assumes that the wave skin-depth $\delta_{\mathrm{P}}$ which is determined by the E-layer Pedersen conductivity $\sigma_{\mathrm{P}}$, namely $\delta_{\mathrm{P}}=\sqrt{2 / \mu_{0} \omega \sigma_{\mathrm{P}}}$, is larger than the thickness of the ionospheric conductive layer $\Delta h, \Delta h \ll \delta_{\mathrm{P}}$. In addition, in the case of fast magnetoacoustic mode incidence, the horizontal wavelength of the wave is to be much larger than the conductive ionospheric layer thickness, $k \Delta h \ll 1$. For ULF waves with the periods longer than $20 \mathrm{~s}$ the thin ionosphere approximation is well justified. Using this approximation, the description of the MHD wave transmission through the inhomogeneous anisotropically-conducting ionospheric plasma can be replaced by the consideration of the wave interaction with a thin layer with height-integrated Pedersen and Hall conductances, $\Sigma_{\mathrm{P}}$ and $\Sigma_{\mathrm{H}}$, located at altitude $h$. 
Fig. 40 Illustration of the distortion upon propagation to the ground of the Alfvén wave resonant structure incident on the ionosphere

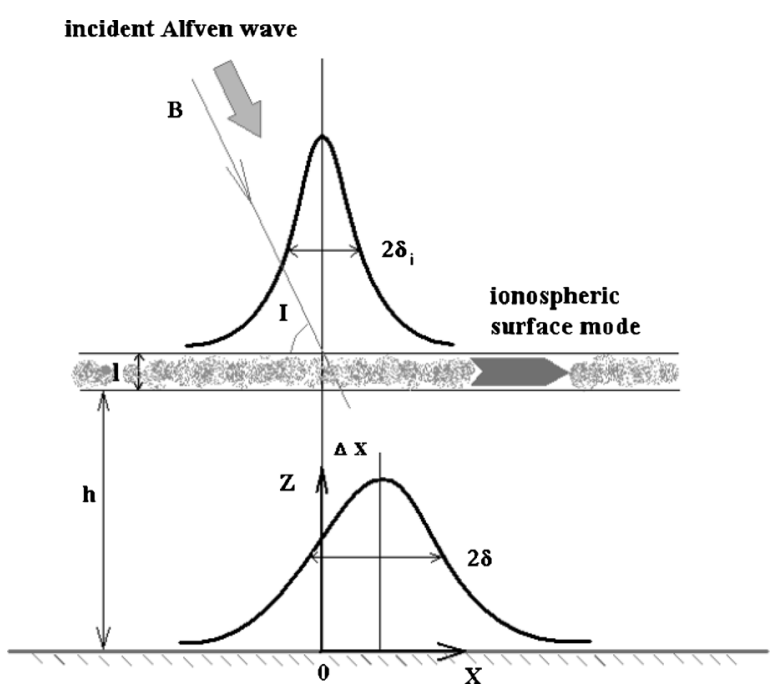

The theory of MHD wave transmission through the thin ionosphere (Hughes and Southwood 1976) gives rather cumbersome analytical expressions for the coefficient $R$ of the Alfvén plane wave reflection and the coefficient $T$ of its transformation into a magnetic mode in the atmosphere. If one neglects the inductive part of the wave electric field in the ionosphere, related to the secondary compressive mode (the induction Hall effect), and the finite electrical conductivity of the Earth's surface, these expressions reduce to the simple forms

$$
R=\frac{b_{y}^{(r)}(\omega, k)}{b_{y}^{(i)}(\omega, k)}=\frac{\Sigma_{\mathrm{P}}-\Sigma_{\mathrm{A}} \sin I}{\Sigma_{\mathrm{P}}+\Sigma_{\mathrm{A}} \sin I}, \quad T=\frac{b_{x}^{(g)}(\omega, k)}{b_{y}^{(i)}(\omega, k)}=\frac{\Sigma_{\mathrm{H}}}{\Sigma_{\mathrm{P}}} \sin I \exp (-k h),
$$

where $I$ is the local inclination angle of geomagnetic field, and the superscripts $(r),(i)$ and $(g)$ denote the reflected and incident magnetospheric waves, and the ground response, correspondingly. The efficiency of the Alfvén wave reflection from the ionosphere, as follows from Eq. (58), is determined by the contrast between the ionospheric Pedersen conductance $\Sigma_{\mathrm{P}}$ and the magnetospheric wave conductance $\Sigma_{\mathrm{A}}=\left(\mu_{0} V_{\mathrm{A}}\right)^{-1}$. The relationship given by Eq. (58) shows that the ground magnetic response to Alfvén waves is controlled by the ratio between the local wave vector $k$ and the height of the ionospheric current layer $h$. Large-scale waves with $k h<1$ produce magnetic response on the ground, whereas smallscale waves with $k h \gg 1$ are screened by the ionosphere from ground-based magnetometers.

More specifically, the ground response to a magnetospheric resonant structure of a spatial scale $\delta_{i}$ is described as

$$
b(x)=\frac{1}{2 \pi} \int_{0}^{\infty} b_{y}(k) T(k) \exp \left(i k x^{\prime}\right) d k \simeq b_{0} \frac{\Sigma_{\mathrm{H}}}{\Sigma_{\mathrm{P}}} \frac{i \delta_{i}}{x^{\prime}+i\left(\delta_{i}+h\right)} \sin I,
$$

where $b_{0}$ is the amplitude of the latitudinal peak maximum. As follows from Eq. (59) the ground magnetic signal keeps the same form as the incident wave, whereas the maximum amplitude changes by the factor $\left(\Sigma_{\mathrm{H}} / \Sigma_{\mathrm{P}}\right) \sin I \delta_{i} /\left(\delta_{i}+h\right)$ and the peak width increases by the factor $\left(\delta_{i}+h\right) / \delta_{i}$. Thus, the existing models of the Alfvén wave transmission through the ionosphere predict that a spatial structure similar to that above the ionosphere should hold 
on the ground. Accordingly, the same relationship (30) could be used, where it is enough to replace $B_{y}$ (magnetosphere) $\rightarrow B_{x}$ (ground) and account for the $\pi / 2$ rotation of the polarisation ellipse.

Numerical modelling of the ground-based ULF spatial structure beneath the field line Alfvén resonance, using the complete theory of MHD wave transmission through a thin ionosphere, including the induction Hall effect and the finite Earth's conductivity, revealed new features: (a) northward shift of the maximum, (b) distortion of its "Lorentz-type" form (see Eq. (30)), and (c) additional shielding of the magnetospheric ULF signal from the ground beneath a high-conductive ionosphere, as illustrated in Fig. 40. From a physical point of view, these effects are related to the excitation of a surface-type mode in the ionosphere. However, these features are of secondary importance and should be most evident at low-latitude observatories, where the resonant Alfvén frequency is high.

Are there any similarities between the chromospheric and ionospheric impact on MHD oscillations? Indeed, coronal loops are rooted into a much more dense, partly-ionised plasma of the chromosphere. How important might be the interaction of the loop oscillations with the underlying chromosphere? How efficient are the wave reflection and absorption? Can the boundary damping compete with the resonant damping of loop oscillations? There have been some dedicated studies performed (e.g. Beliën et al. 1999, see also Sect. 10.3), but a much more systematic effort is need to reveal the role of the chromosphere on MHD oscillations in the corona.

\subsection{Short-Period Alfvén Wave Interaction with a Realistic Ionosphere}

For low frequencies (periods longer than $20 \mathrm{~s}$ ) the approximation of the thin layer ionosphere may be used for the description of MHD wave interactions with ionosphere. However, this theory cannot be used for waves in a higher frequency range, whose field-aligned wavelengths are comparable to the vertical scale of the ionospheric inhomogeneity.

A characteristic feature of the upper ionosphere is the occurrence of the IAR and the fast magnetoacoustic waveguide (Sect. 3.8.1), which can trap MHD wave energy in the range from fractions of a $\mathrm{Hz}$ to a few $\mathrm{Hz}$. The occurrence of the IAR and fast magnetoacoustic waveguide ensures the strong dependence of the ionospheric transmission/reflective properties of incident Alfvén waves on the frequency (Ostapenko and Poliakov 1990). The ionosphere can act as a multi-band band-pass filter for magnetospheric MHD waves, and determines, together with the magnetospheric wave source, the spectral structure observed on the ground. Numerical models accounting for the Alfvén wave interaction with a realistic ionosphere, predicted that at nighttime the transmission coefficient $T(f, k)$ of Alfvén waves to the ground has an oscillatory dependence on frequency, with many narrow "transmission windows" at the IAR eigenfrequencies. For a broadband magnetospheric emission the occurrence of the "transparency windows" may result in the formation of narrow-band emissions observed on the ground after transmission through the ionosphere.

A typical model describing the propagation of MHD waves through the magnetosphereionosphere-atmosphere system to the ground is a set of multiple layers with different electrodynamic parameters, permeated by the geomagnetic field $\mathbf{B}_{0}$ with inclination $I$. An incidence of Alfvén waves with various frequencies and transverse scales, azimuthally extended waves with $k_{2} \ll k_{1}$, is considered, where $k_{1}$ and $k_{2}$ are the effective radial (latitudinal) and azimuthal (longitudinal) wave numbers. The wave structure is determined from the solution to Maxwell's equations with the complex tensor permittivity $\hat{\varepsilon}_{\perp}(\omega, z)$, comprising the local Pedersen and Hall conductivities, $\sigma_{\mathrm{P}}(z, \omega)$ and $\sigma_{\mathrm{H}}(z, \omega)$. In an oblique-angled coordinate 
Fig. 41 Reflection $R(f)$ and transmission $T(f)$ coefficients for Alfvén waves with the wave numbers $k_{\perp}=10^{-3} \mathrm{~km}^{-1}$ (dark line) and $k_{\perp}=10^{-2} \mathrm{~km}^{-1}$ ( grey line), interacting with the nightside ionosphere at mid-latitude $\left(I=58.6^{\circ}\right)$

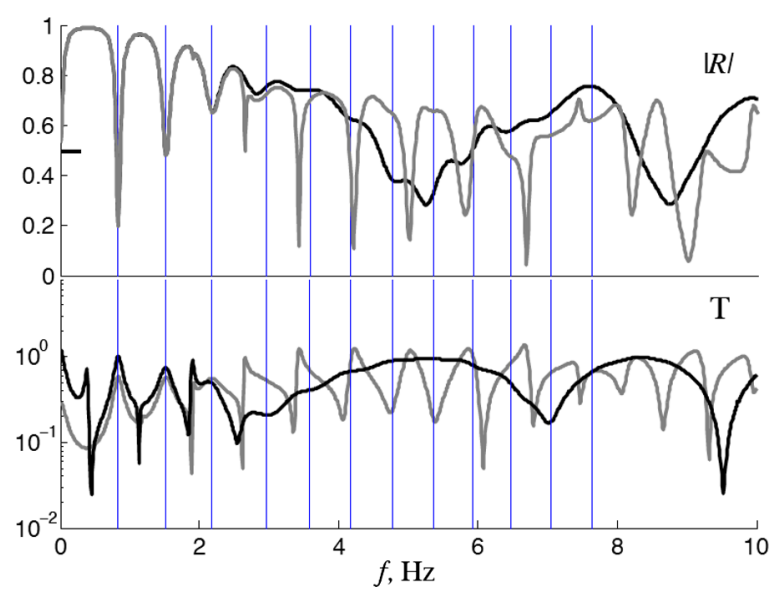

system under the assumptions $\sigma_{\|} \rightarrow \infty$ and $k_{2}=0$, with $k \equiv k_{1}$, Maxwell's equations are reduced to the following set of equations:

$$
\begin{aligned}
& \partial_{3} b_{1}=i k \cot I b_{1}-\mu_{0} \sigma_{\mathrm{H}}(\sin I)^{-1} e_{1}+i \omega^{-1}\left(k^{2}-i \mu_{0} \omega \sigma_{\mathrm{P}}\right) e_{2}, \\
& \partial_{3} b_{2}=-\mu_{0} \sigma_{\mathrm{P}}(\sin I)^{-2} e_{1}-\mu_{0} \sigma_{\mathrm{H}}(\sin I)^{-1} e_{2}, \\
& \partial_{3} e_{1}=i \omega b_{2}, \quad \partial_{3} e_{2}=-i \omega b_{1}+i k \cot I e_{2},
\end{aligned}
$$

where $e_{1,2}$ and $b_{1,2}$ are the perturbations of the electric and magnetic fields, respectively. If the Hall conductivity $\sigma_{\mathrm{H}}$ can be neglected, then the set of equations (61) splits into two uncoupled sub-sets, for Alfvén waves (with the non-vanishing components $e_{1}$ and $b_{2}$ ), and for fast magnetoacoustic waves (with components $e_{2}$ and $b_{1}$ ).

For terrestrial applications, the altitude profile of the ionospheric parameters given by the International Reference Ionosphere (IRI) model is used. Using the altitude profiles of the electron and ion collision frequencies, $v_{\mathrm{e}}$ and $v_{\mathrm{i}}$, Alfvén speed $V_{\mathrm{A}}(z)$, the plasma conductivities $\sigma_{\mathrm{P}}(z, \omega)$ and $\sigma_{\mathrm{H}}(z, \omega)$, one can calculate the refraction index $n_{\mathrm{A}}(z)=\operatorname{Re} \sqrt{\varepsilon_{\mathrm{P}}}$, $\varepsilon_{\mathrm{P}}=i \sigma_{\mathrm{P}} /\left(\varepsilon_{0} \omega\right)$.

During the interaction with the anisotropically-conducting ionosphere, Alfvén and fast magnetoacoustic modes are linearly coupled. Therefore, the reflection and transmission matrices are needed for the description of the coupling of the horizontal components of the magnetic fields in the incident $(i)$ and reflected $(r)$ waves. Further, we present the results of the incidence and reflection of Alfvén mode only, characterised by the reflection coefficient $R(f)$ (element of the reflection matrix), the ratio of the horizontal magnetic components of reflected and incident Alfvén wave $R=b_{2}^{(r)} / b_{2}^{(i)}$, and the transmission coefficient, which is defined as the ratio between the amplitudes of magnetic field disturbance on the ground and magnetic component of an incident Alfvén wave $T=b_{1}^{(g)} / b_{2}^{(i)}$

As an example, we show results of the numerical modelling for typical mid-latitude location during nighttime (Fig. 41). To reconstruct the altitude profile of the plasma conductivities $\sigma_{\mathrm{P}}(z, \omega)$ and $\sigma_{\mathrm{H}}(z, \omega)$ and refraction index $n_{\mathrm{A}}(z)$, the IRI and Mass-SpectrometerIncoherent-Scatter (MSIS) models ${ }^{3}$ were used. During nighttime the height-integrated (60$400 \mathrm{~km}$ ) conductances are $\Sigma_{\mathrm{P}}=0.26 \mathrm{~S}$ and $\Sigma_{\mathrm{H}}=0.17 \mathrm{~S}$, whereas during daytime the

\footnotetext{
${ }^{3}$ http://ccmc.gsfc.nasa.gov/modelweb/.
} 
conductances are $\Sigma_{\mathrm{P}}=10.0 \mathrm{~S}$ and $\Sigma_{\mathrm{H}}=10.56 \mathrm{~S}$. We consider the features of reflection and transmission through a realistic IRI-derived ionosphere of Alfvén waves with various transverse scales, from $k=10^{-3} \mathrm{~km}^{-1}$ to $k=10^{-1} \mathrm{~km}^{-1}$.

The effect of the IAR and fast magnetoacoustic waveguides on the ionospheric reflection coefficient $R(f)$ as a function of the frequency is clearly visible in Fig. 41, upper panel. An important issue is the modification of the spectral content of the magnetospheric noise and waves upon transmission through the ionosphere to the ground, characterised by the transmission coefficient $T(f, k)$. The coefficient $T(f)$ has a strong oscillatory dependence on the frequency, with maxima at the IAR eigenfrequencies with $f<4 \mathrm{~Hz}$ (Fig. 41, bottom panel). At $f>3 \mathrm{~Hz}$ the behaviour is drastically different for different scales: for $k_{\perp}=10^{-2} \mathrm{~km}^{-1} T(f)$ it is oscillatory till rather high $f \simeq 10 \mathrm{~Hz}$, whereas for $k_{\perp}=10^{-3} \mathrm{~km}^{-1}$ broadband "transmission windows" appear at higher frequencies, namely 4-6 Hz. This "transmission window" may result in an enhanced magnetospheric noise leakage to the ground in this frequency band.

For very small scales, a collisional field-aligned conductivity $\sigma_{\|}$(neglected in the above model) should be taken into account. The collisional damping in the ionosphere leads to absorption of Alfvénic waves with wave numbers larger than $k_{\perp}^{*} \simeq \mu_{o} \sigma_{\|} / \tau_{\mathrm{A}}$, where $\tau_{\mathrm{A}}$ is the Alfvénic time. According to Lessard and Knudsen (2001), Alfvén waves with spatial scales less than a few $\mathrm{km}$ are strongly diffused and damped upon the reflection from the realistic ionosphere. This effect limits the transverse scale of IAR from below. The possible occurrence in the ionosphere of small-scale Alfvénic structures with the transverse scales comparable to the dispersive scale (typically, about few hundred $\mathrm{m}$ ), commonly invoked to interpret the mechanism for the auroral electron acceleration, is still disputable.

\subsection{The Role of Partially Ionised Plasma in Coronal MHD Oscillations}

The possible damping effects of partially ionised plasma on MHD waves observed in the corona is of natural interest to solar physicists. Thus far, such studies have mainly been motivated by observations of fast kink waves in coronal prominences (e.g., Oliver and Ballester 2002; Lin et al. 2009; Hillier et al. 2013) and loops (e.g., Aschwanden et al. 1999; Nakariakov et al. 1999). Prominences are complex and rather mysterious filamentary magnetic structures of dense chromospheric temperature plasma embedded in the much more diluted and hotter corona (the prominence material is about 100 times cooler and 100 times denser than the surrounding corona; for a recent review see Parenti 2014). The width of resolved prominence threads is about $200 \mathrm{~km}$ and is near the current resolution limit of ground and space-based instruments in spectral lines formed at chromospheric temperatures (the order of $10^{4}-10^{5} \mathrm{~K}$ ), e.g., $\mathrm{H} \alpha, \mathrm{H} \beta$, Ca II $\mathrm{K}$ and $\mathrm{Ca}$ II $\mathrm{H}$. The observed length of these threads can vary between $3.5-28 \mathrm{Mm}$, hence comparison of their width and length permits us to classify them as thin flux tubes (see review by Lin 2011). Although exact processes of prominence formation and their filamentation and evolution are still somewhat of a mystery (see Labrosse et al. 2010, for a review), from an MHD waves point of view prominences allow us one of the few opportunities to study partially ionised waveguides at coronal altitudes. With regard to partial ionisation effects in loops, the effect occurs only at their footpoint regions. The much larger portion of their length is in the highly ionised corona but they are rooted in the neutral photosphere and partially ionised chromosphere. To quantify the difference in the chromospheric and coronal arc lengths of loops, the chromospheric arc length extends for only about $2 \mathrm{Mm}$, while the coronal arc length is often of the order of $100 \mathrm{Mm}$. Hence, the partially ionised arc lengths of coronal loops only represent a few percent at most of their total arc lengths. 
Modelled values of ion-neutral collisional frequencies in solar atmospheric plasma of chromospheric temperature and density cover a rather large range, between $10-10^{4} \mathrm{~Hz}$ (e.g., Martínez-Sykora et al. 2012). In comparison, observed waves are in the sub-Hz regime in both prominences and loops. There have been a significant number of studies measuring periods of, in particular, kink waves in prominence threads (see review by Lin 2011). They are mostly in the range of a few minutes, but some much longer periods have been measured in the 10-100 minute range by e.g., Terradas et al. (2002) and Hillier et al. (2013), and even can reach several hours (Hershaw et al. 2011). As previously discussed in Sect. 6.1 typical standing fast kink mode periods in coronal loops are also from a few minutes to a few tens of minutes. Longitudinal modes of coronal loops (Sect. 6.3) are observed to have periods longer than several minutes too. Sausage modes (Sect. 6.2) have shorter periods, but there is no observational evidence that the periods are shorter than $0.5 \mathrm{~s}$. Propagating coronal waves (Sect. 7) have periods longer than $1 \mathrm{~s}$ too.

Consider the effect of partial ionisation on coronal MHD waves on the example of kink modes of coronal plasma structures and the torsional Alfvén waves. Let $\omega$ be the cyclic frequency of a fast kink wave propagating through a plasma with the ion-neutral collisional frequency $v_{\text {in }}$. From observations and expected conditions of partially ionised plasma in the solar atmosphere it is reasonable to assume that $\omega \ll v_{\text {in }}$. Therefore the effect of strong collisional coupling between ions and neutrals cannot be ignored. Due to the much smaller momentum of electrons their collisions with ions and neutrals is usually neglected (e.g., see Soler et al. 2009). Note that the inclusion of electron inertia is crucial for modelling other wave-based phenomena, e.g., electron acceleration due to Alfvén waves in both the magnetospheric (e.g., Goertz and Boswell 1979; Chaston et al. 2002b) and solar context (e.g., Fletcher and Hudson 2008). However, for MHD wave modes propagating in partially ionised regions of the Sun's atmosphere it is the small but finite coupling time between ions and neutrals that is most important, causing a frequency-dependent wave damping mechanism (e.g., de Pontieu and Haerendel 1998; De Pontieu et al. 2001; Soler et al. 2009, 2011, 2012) that is quite distinct from that of resonant absorption (as discussed in Sect. 5.5). This ion-neutral coupling also results in an increased inertia of the plasma, experienced by MHD waves from the combined ion and neutral densities. To quantify the amount of ionisation in the plasma let

$$
\xi_{\text {in }}=\frac{\rho_{\mathrm{n}}}{\rho_{\mathrm{i}}}
$$

where $\rho_{\mathrm{n}}$ and $\rho_{\mathrm{i}}$ are the neutral and ion densities, respectively. So here $\xi_{\text {in }}=0$ refers to a fully ionised plasma and $\xi_{\text {in }}=\infty$ denotes a neutral plasma.

In cylindrical flux tube geometry (Sect. 4) the effect of ion-neutral damping has been modelled for various MHD wave modes (e.g., Soler et al. 2009). Here we consider only the $m=0$ torsional Alfvén wave and the $m=1$ fast kink wave. As shown by de Pontieu and Haerendel (1998), for torsional Alfvén waves the damping length due to ion-neutral coupling is

$$
L_{\mathrm{D}, \text { in }}=2 \bar{V}_{\mathrm{A}} \frac{\left(1+\xi_{\text {in }}\right)}{\xi_{\text {in }}} \frac{v_{\text {in }}}{\omega^{2}}
$$

where

$$
\bar{V}_{\mathrm{A}}=\frac{B}{\sqrt{\mu_{0}\left(\rho_{\mathrm{n}}+\rho_{\mathrm{i}}\right)}}
$$

is the modified Alfvén speed due to the increased inertia from the ion-neutral coupling. It can be seen from Eq. (63) that the damping length due to ion-neutral collisions is inversely 
proportional to $\omega^{2}$. Soler et al. (2012) derived the equivalent expression for the fast kink wave, assuming that $\xi_{\text {in }}$ is the same inside and outside the tube, i.e.,

$$
L_{\mathrm{D}, \text { in }}=2 \bar{V}_{\mathrm{K}} \frac{\left(1+\xi_{\text {in }}\right)}{\xi_{\text {in }}} \frac{v_{\text {in }}}{\omega^{2}} .
$$

Here $\bar{V}_{\mathrm{K}}$, the modified kink speed, is defined the same as in Eq. (26) but with $\rho_{0}$ replaced by $\rho_{0, \mathrm{i}}+\rho_{0, \mathrm{n}}$, the sum of ion and neutral densities inside the tube, and $\rho_{\mathrm{e}}$ is replaced by $\rho_{\mathrm{e}, \mathrm{i}}+\rho_{\mathrm{e}, \mathrm{n}}$, the sum of ion and neutral densities outside the tube. Soler et al. (2012) went further to study the combined effects of damping due to ion-neutral collisions and resonant absorption together. Assuming a thin resonant layer $(l / a \ll 1)$ as was done for Eq. (40) in Sect. 5.5. Soler et al. (2012) found that the damping length $L_{\mathrm{D}}$, due to the two effects could be written as

$$
\frac{1}{L_{\mathrm{D}}}=\frac{1}{L_{\mathrm{D}, \mathrm{in}}}+\frac{1}{L_{\mathrm{D}, \mathrm{ra}}},
$$

where $L_{\mathrm{D} \text {,ra }}$ is the damping length due to resonant absorption in a partially ionised plasma, i.e.,

$$
L_{\mathrm{D}, \mathrm{ra}}=\frac{2}{\pi} \bar{V}_{\mathrm{K}} \frac{a}{l} \frac{\rho_{0}+\rho_{\mathrm{e}}}{\rho_{0}-\rho_{\mathrm{e}}} \frac{1}{\omega} .
$$

Here the factor $2 / \pi$ comes from the fact a sinusoidal variation in density is taken between the internal and external plasma as in Eq. (40) in Sect. 5.5. Note that the damping length due to resonant absorption is inversely proportional to $\omega$, consistent with the results of Terradas et al. (2010) and Verth et al. (2010) in the fully ionised case. Taking the ratio of the damping lengths given in Eqs. (65) and (67) we have that

$$
\frac{L_{\mathrm{D}, \mathrm{ra}}}{L_{\mathrm{D}, \mathrm{in}}} \propto \frac{\xi_{\text {in }}}{1+\xi_{\text {in }}} \frac{\omega}{v_{\text {in }}} .
$$

The largest value the factor $\xi_{\text {in }} /\left(1+\xi_{\text {in }}\right)$ can take is unity for the limit at $\xi_{\text {in }}=\infty$, i.e. a neutral plasma. Hence for observed kink wave frequencies $\omega \ll v_{\text {in }}$, it follows that $L_{\mathrm{D}, \mathrm{ra}} \ll$ $L_{\mathrm{D} \text {,in }}$, i.e., damping due to resonant absorption dominates over that due to the effect of ionneutral collisions.

There have been various analytical and numerical models developed to investigate Alfvén wave damping in coronal loops due to the inclusion of a chromospheric layer (e.g., Hollweg 1984; Berghmans and de Bruyne 1995; De Pontieu et al. 2001; Ofman 2002). The particular 1.5D numerical model of Alfvén waves by Ofman (2002) did not include the effect of partial ionisation but did have realistic density stratification in the chromospheric layer. It was found that the resulting footpoint leakage caused damping times that were a factor of 5 longer than observed. This agrees with the original conclusions by Nakariakov et al. (1999) that footpoint leakage was not responsible for the observed damping times. Therefore the additional inclusion of partial ionisation in the chromospheric layer for realistic standing kink mode frequencies would have a negligible effect on these results. Anisotropic (Hall and Pedersen) conductivities are usually not considered as the major factor affecting the dynamics of coronal MHD waves, while this phenomenon has been intensively studied in the context of chromospheric waves which are out of scope of this review. On the other hand, this effect may be important in rarefied parts of the corona, e.g. in coronal holes.

Of course, MHD wave damping due to ion-neutral collisions is relevant when $\omega \approx v_{\text {in }}$. It has recently been postulated by Russell and Fletcher (2013), that high frequency (or sub- 
second) Alfvén waves generated by flares in the corona may propagate down to the chromosphere where they will dissipate efficiently due to ion-neutral collisions. This may have important consequences for post-flare chromospheric heating, however, currently due to instrumental limitations it is not possible to observe such high-frequency waves directly.

\section{Quasi-Periodic Pulsations in Solar Flares}

\subsection{Standard Model of a Solar Flare}

Generally, solar flares are visible as a sudden increase in the brightness of the Sun (or a part of the solar surface, if the observational instrument allows for spatial resolution) in all observational bands, from radio to gamma-rays. Physical mechanisms responsible for the radiation vary from bremsstrahlung (for hard X-rays and gamma-rays, visible light) and gyrosynchrotron (microwaves) of non-thermal electrons accelerated by the flare trigger, to thermal emission of the hot (up to several tens of million $\mathrm{K}$ ) plasma evaporated from the denser layers of the solar atmosphere or heated directly in the corona (UV, EUV, microwaves and soft X-rays). Also, in the gamma-ray band the emission is likely associated with nuclear processes, e.g., de-excitation, neutron capture, and positron annihilation, caused by accelerated ions.

During the development of a flare, different physical mechanisms dominate in the emission. According to the timing of the emission recorded in different bands one can distinguish between several phases of a typical solar flare (see, e.g. Benz 2008, for a comprehensive review). In the so-called "preflare phase" one observes increasing EUV and soft X-ray radiation emitted by a gradually heated plasma of the active region where the flare occurs. Later on, a large number of electrically charged particles gets accelerated at the magnetic reconnection site in the active region, causing the "impulsive phase" that typically lasts up to a few minutes only. In this phase hard X-ray, gyrosynchrotron microwave, and gamma-ray emissions rise rapidly, often with many short but intense spikes of emission, each lasting from a fraction of a second to tens of seconds. Some portion of accelerated particles is trapped in the magnetic field of the flaring active region, and gyrates around magnetic field lines producing the emission in the microwave band by the gyrosynchrotron mechanism. The following "flash phase" is characterised by the maxima of soft X-ray and visible light (in particular, $\mathrm{H} \alpha$ that comes from the chromosphere) emission. After that the coronal plasma gradually repairs its initial state in the "decay phase". However the consequent development of a flare still could be recognised in the solar corona as the follow-up magnetic reconfiguration, plasma ejections, various decaying oscillations of various coronal plasma structures, radio bursts, and occasionally as sunquake ripples on the solar surface.

The schematic sketch of the so-called standard (or "Carmichael, Sturrock, Hirayama, Kopp-Pneuman", CSHKP, named after its creators) model of a solar flare, based on the observational phenomenology described above, is shown in Fig. 42. Initially, the magnetic energy is accumulated in active regions of the corona in a form of non-potential magnetic fields. Then some process triggers fast magnetic reconnection in, e.g. the current sheet formed behind of a destabilised ascending plasmoid, causing a rapid release of the free magnetic energy. The current sheet is oriented vertically, somehow resembling the tail in the Earth's magnetosphere. The magnetic energy is converted to the internal energy of the plasma, kinetic energy of bulk flows, e.g. Alfvénic jets or plasmoids, and still poorly understood acceleration of the super-thermal population of charged particles (also called non-thermal, accelerated or energetic particles), usually having the peaking energies in the 
Fig. 42 Schematic sketch of the standard model of a solar flare

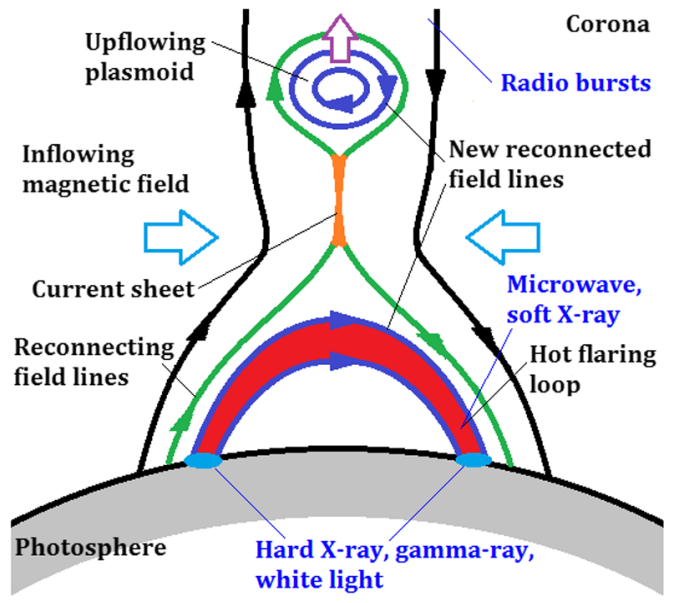

vicinity of $20 \mathrm{keV}$ and extending sometimes to several tens of $\mathrm{MeV}$. The accelerated charged particles move along the field lines, emitting the gyrosynchrotron radiation in the microwave band. The electrons that move towards the solar surface then precipitate into the dense plasma of the lower chromosphere that offers them a thick target. The precipitating nonthermal particles produce the hard X-ray and gamma-ray bremsstrahlung and nuclear emission. The chromospheric material gets heated up to tens of million $\mathrm{K}$ and evaporates upward into the corona. This evaporated thermal plasma fills up coronal magnetic flux tubes (coronal loops), emitting soft X-rays. At this stage one can see the appearance of bright "post-flaring" coronal loops in the soft X-ray band. The non-thermal electrons that move upwards interact with the background thermal plasma (see, e.g., the discussion in Sect. 9.2.1) producing various high-frequency radio bursts. This standard model of a flare should be taken as the zero-order approximation only, as it does not include 3D effects. In particular, major solar flares are usually seen to be "two-ribbon": the energy release sites move across the magnetic field, along the neutral line on the surface of the Sun, that separates the footpoints of the opposite magnetic polarity. On the surface of the Sun the sources of the hard X-ray or visible light emission form two "ribbons" that are extended along the magnetic neutral line. In the corona the ribbons are connected by an arcade consisting of a number of isolated loops parallel each other. There are ongoing attempts to generalise the standard model of a flare accounting for 3D effects (see, e.g. Aulanier et al. 2012).

\subsection{Observational Manifestation of QPPs}

An interesting feature of flaring energy releases are quasi-periodic pulsations (QPPs) often seen in light curves of solar flares as well-pronounced modulation of the emission intensity (see Nakariakov and Melnikov 2009, for a detailed review). These periodic variations of the electromagnetic emission are observed in all bands: the radio, visible light, soft and hard X-rays, and gamma-rays. Typical periods of solar flaring QPPs range from a fraction of a second to several minutes. Statistical studies show that QPPs are a common and perhaps intrinsic feature of flares (Kupriyanova et al. 2010). An example of QPPs is shown in Fig. 43 where the period is of about $40 \mathrm{~s}$, and QPPs are almost synchronous in all observation bands. Moreover it often appears that the periods of QPPs are not stable, and vary during the same event (Nakariakov et al. 2010a; Kupriyanova et al. 2010; Kolotkov et al. 2015). Likewise, QPPs may have well-pronounced amplitude modulation. Usually, the most 



Fig. 43 Time development of a typical solar flare with QPPs of the microwave, hard X-ray and gamma-ray emission. (a) Intensity of $17 \mathrm{GHz}$ (solid) and $34 \mathrm{GHz}$ (dashed) emission recorded with the Nobeyama Radioheliograph, and hard X-ray emission at 42-80 keV (thick) recorded with CORONAS-F/SONG; (b) 80-225 $\mathrm{keV}$ (thin) recorded with CORONAS-F/SONG, and 50-100 keV (dashed), and 100-300 keV (thick) recorded with RHESSI; (c) 80-225 keV (thick), 225-750 keV (dashed), and 2-6 MeV (thin solid) recorded with CORONAS-F/SONG; the dashed curve shows the averaged $2-6 \mathrm{MeV}$ light curve from previous orbits. The elapsed time is measured from 00:00 UT on 1 January 2005. (d) Photon energy spectrum integrated between 00:28 UT and 00:32 UT obtained with RHESSI. The light curves are normalised to their maximum values. From Nakariakov et al. (2010b)

pronounced QPPs are seen in the emission associated with non-thermal electrons, e.g. the gyrosynchrotron emission in the microwave band and bremsstrahlung in the hard X-ray. However QPPs are also detected in the soft X-ray radiation produced by thermal free-free emission. QPPs can be found at all stages of the flare development: from the preflare to decay phases.

In some cases, analysis of QPPs shows the co-existence of several statistically-significant peaks. For example, Inglis and Nakariakov (2009) found three spectral peaks, at $28 \mathrm{~s}, 18 \mathrm{~s}$, and $12 \mathrm{~s}$, in the microwave emission produced by a flare. The $28 \mathrm{~s}$ and $18 \mathrm{~s}$ oscillations were also detected in the hard X-ray $40-60 \mathrm{keV}$ energy range. The ratios of the periods do not allow to attribute them to the effect of nonlinear doubling of frequency.

The importance of research in QPPs is not only connected with the physics of energy releases, but also with the uniquely high time resolution of the observations, offered by the high intensity of the flaring emission. Moreover, there is a growing interest in QPPs detected in stellar flares and their use for seismological diagnostics.

It is commonly accepted that QPPs can be produced by several non-exclusive mechanisms, such as modulation of the plasma parameters by MHD waves (Nakariakov and Melnikov 2009; Stepanov and Zaitsev 2014), modulation of the non-thermal charged particle kinematics by MHD waves (Zaitsev and Stepanov 1982), periodic triggering of energy releases by external MHD waves (Nakariakov et al. 2006), MHD flow over-stability (Ofman and Sui 2006), alternate currents in effective LCR-circuits equivalent to current-carrying 
loops (Khodachenko et al. 2009), and self-oscillatory (or repetitive) regimes of magnetic reconnection (Kliem et al. 2000). Short period QPPs, in the sub-second period range, could be also associated with wave-particle interactions (Aschwanden 1987). Here, we concentrate our attention on medium- and long-period, longer than one second, QPPs only.

\subsection{Modulation of Flaring Emission by MHD Waves}

MHD waves can directly modulate flaring emission by perturbations of the background plasma. Consider, for example, the gyrosynchrotron emission produced by mildly relativistic electrons, accelerated in solar flares. Generally the intensity $I_{\mathrm{f}}$ of the gyrosynchrotron emission at a certain frequency $f$ has a peak value at some peak frequency $f_{\text {peak }}$ (typically $f_{\text {peak }}>10 \mathrm{GHz}$ ) and determined by macroscopic parameters of the plasma, such as the absolute value of the background magnetic field $B$ and the electron concentration $n_{0}$ in the loop, the angle $\theta$ between the line-of-sight and the magnetic field; and by the characteristics of the injected population of non-thermal particles: their total number $N_{\mathrm{NT}}$ in the emitting volume, the energy power-law index $\delta_{\mathrm{NT}}$ (which is usually $>3-5$ ) and pitch-angle anisotropy of the non-thermal electron distributions (see, e.g. Mossessian and Fleishman 2012, and references therein). The gyrosynchrotron radiation in flaring sites can occur in both optically thin (when the plasma is transparent to the radiation) and optically thick regimes (when the detected radiation comes from a certain surface behind which the plasma is opaque to the radiation). Usually the optically thin regime is observed at frequencies higher than the gyrosynchrotron peak frequency $f_{\text {peak }}$, while the optically thick emission radiates at lower frequencies, $f<f_{\text {peak }}$. In the different regimes the intensity $I_{\mathrm{f}}$ depends upon the parameters of the background plasma and the population of non-thermal electrons differently, i.e.

$$
I_{\mathrm{f}} \propto \begin{cases}N_{\mathrm{NT}} B^{0.9 \delta_{\mathrm{NT}}-0.22} & \text { in optically thin regime, } \\ B^{-0.5-0.09 \delta_{\mathrm{NT}}} & \text { in optically thick regime. }\end{cases}
$$

For $\delta_{\mathrm{NT}}>0.25$ the increase in the magnetic field in the MHD wave leads to the increase in the emission intensity in the optically thin regime, and decrease in the intensity in the optically thick regime. In the optically thin case the gyrosynchrotron radiation is especially sensitive to the variations of the magnetic field amplitude $B$, due to the strong power-law dependence. In particular, for $\delta_{\mathrm{NT}}=4$ this proportionality becomes $I_{\mathrm{f}} \propto B^{3.4}$. Hence even relatively low perturbations of the magnetic field, typical for MHD waves observed in the corona, can cause significant modulation of the observed gyrosynchrotron signal. In particular, sausage oscillations (see Sect. 4), disturbing the magnetic field strength, are able to produce significant modulation of the microwave gyrosynchrotron emission in the optically thin part of the spectrum (e.g. Reznikova et al. 2014).

Similarly, consider the dependence of the intensity of the gyrosynchrotron emission upon the angle $\theta$ between the LOS and magnetic field

$$
I_{f} \propto \begin{cases}(\sin \theta)^{-0.43+0.65 \delta_{\mathrm{NT}}} & \text { in optically thin regime, } \\ (\sin \theta)^{-0.36-0.06 \delta_{\mathrm{NT}}} & \text { in optically thick regime. }\end{cases}
$$

In the optically thin regime small-amplitude variations of the angle $\theta$, generated, e.g. by a kink or torsional standing modes or propagating waves, are amplified according to Eq. (70) for $\delta_{\mathrm{NT}}>0.67$. For the typical values, $\delta_{\mathrm{NT}}>3$ the relative signal of the MHD oscillation, presented in the modulation of the gyrosynchrotron emission, is significantly amplified. Similarly to Eq. (69), signals from optically thick part of the gyrosynchrotron spectrum 
show an anti-phase behaviour (Mossessian and Fleishman 2012). The recently observed correlation between the microwave flux and the spatial separation of two microwave sources, observed with NoRH, can be the manifestation of the vertically polarised global kink mode of the flaring loop (Kupriyanova et al. 2013).

Consequently, fast magnetoacoustic waves are readily able to produce significant quasiperiodic modulation of the gyrosynchrotron emission intensity at the microwave frequencies. Moreover, slow magnetoacoustic waves can also cause quasi-periodicity by the modification of the plasma density and hence the electron plasma frequency. That is manly pronounced at lower frequencies, $f<10 \mathrm{GHz}$, which are lower than $f_{\text {peak }}$ (Nakariakov and Melnikov 2006). Usually this part of the gyrosynchrotron spectrum is optically thick for radiation, but it becomes optically thin in the case of relatively dense plasma due to the effect of Razin suppression. This effect happens quite often in observed flaring loops (see, e.g. Melnikov et al. 2008). The threshold frequency, termed as the Razin frequency, can be estimated as $f_{\text {Razin }}=\omega_{\text {pe }}^{2} / 6 \pi \Omega_{\mathrm{i}}$, which is typically in the vicinity of 5-10 GHz, but in some exotic cases reaches even $20 \mathrm{GHz}$. In case of periodic variations of the plasma concentration $n_{0}$, by, e.g. a compressive longitudinal (slow magnetoacoustic, in the low- $\beta$ typical for flaring active regions) wave in which the magnetic field remains almost constant, the Razin frequency does also experience oscillations. It would result in the periodic modulation of the produced microwave radiation. The modulation amplitude can become up to ten times higher than the relative amplitude of the modulating MHD wave.

Likewise, modulation of the main plasma parameters by an MHD wave causes modulation of the coherent radio emission in the metric and decimetric bands, generated by the interaction of non-thermal particles accelerated in solar flares with the background plasma. The non-thermal particles excite the Langmuir waves by, e.g. the bump-on-tail instability, which in turn are converted in electromagnetic waves coming to the ground-based or spaceborne receiver.

Thus, all main MHD modes (sausage, kink, and longitudinal, see Sect. 4) of coronal plasma structures, and torsional Alfvén waves can produce quasi-periodic modulation of the microwave emission of solar flares by direct perturbation of the macroscopic parameters of the emitting plasma. Moreover, the observed amplitude of the emission intensity variation (the modulation depth) can be several times higher than the amplitude of the modulating oscillation. However, in some cases the observed modulation depth reaches $50 \%$ or even $100 \%$, which is difficult to interpret in terms of this mechanism. In addition, this mechanism does not explain the simultaneous and coherent QPPs often observed in different bands. For example, panel (a) of Fig. 43 shows that QPPs in the radio emission correlate well with the modulation of the hard X-ray and gamma-ray curves. In the standard model of a solar flare (Sect. 11.1) hard X-ray and gamma-ray emission is associated with the footpoints sources, generated by the bremsstrahlung of highly energetic electrons in the dense chromospheric plasma, while the gyrosynchrotron emission is produced by the same non-thermal electrons above the footpoints, in the loop legs. Simultaneous QPPs of hard X-ray and microwave emission can be explained in terms of the modulation of non-thermal charged particle trajectories by an MHD oscillation.

A significant portion of accelerated particles is confined inside the magnetic trap made by the magnetic flux tube of a non-uniform cross-section, representing the flaring loop. Indeed, as the cross-sectional area of flaring loops increases with height reaching the maximum at the loop top, the magnetic field decreases from the footpoints to the loop top. A very similar magnetic configuration can be found in the closed magnetosphere. The trapped non-thermal electrons have pitch angles greater than the critical value $\alpha_{\text {crit }}$, defined as $\sin ^{2} \alpha_{\text {crit }}=B_{\text {top }} / B_{\text {fp }}$, where $B_{\text {top }}$ and $B_{\text {fp }}$ are the values of the magnetic field at the loop 
top and the loop footpoints, respectively, typically $B_{\mathrm{top}} \leq B_{\mathrm{fp}}$. The legs of the magnetic flux tube, where its cross-sectional area decreases, act as magnetic mirrors for the population of the non-thermal electrons near the loop top. In the case of periodic variations of the mirror ratio, $B_{\mathrm{top}} / B_{\mathrm{fp}}$, e.g., by the sausage oscillation, the critical value of the pitch angle $\alpha_{\text {crit }}$ periodically changes, allowing periodic passage of the accelerated electrons through the magnetic mirrors and consequent precipitation at the dense plasma layers at the footpoints. This process, known as the Zaitsev-Stepanov mechanism, can lead to QPPs of the hard X-ray emission from the footpoint sources (Zaitsev and Stepanov 1982). Simultaneously, this scenario produces QPPs of the gyrosynchrotron emission generated by the non-thermal electrons that periodically filling in the loop's legs. A similar effect can be also produced by a slow magnetoacoustic mode of the sausage $(m=0)$ symmetry, if the plasma $\beta$ is sufficiently large. It should also be noted, that the Zaitsev-Stepanov mechanism requires further detailed investigation and modelling.

\subsection{Periodic Triggering of Energy Releases by External MHD Oscillations}

Another possibility for MHD-wave-driven QPPs in solar flares is a periodic triggering of the primary energy release, the reconnection of the magnetic field lines, by external MHD waves. These waves may be MHD oscillations in nearby plasma structures, e.g. a neighbouring loop, or may be approaching propagating fast waves generated elsewhere. For example, the periodically varying inflow of the plasma with the frozen-in magnetic field should modulate the reconnection rate in the current sheet or X-point. Moreover, such a mechanism does not require the driving external MHD oscillation to be of large amplitude, due to essentially nonlinear effect of the triggering.

A detailed model of the periodic modulation of magnetic reconnection by transverse MHD waves, such as, in particular, kink or sausage modes, was designed by (Nakariakov et al. 2006, see the sketch in Fig. 44). The external oscillation can be either in the trapped or leaky regimes. Hence, the linkage of this oscillation with the flaring site is provided by the evanescent or leaky part of the oscillation, outside the wave guiding plasma nonuniformity. From the point of view of the flaring site, this external transverse oscillation looks like periodically incoming and outgoing perpendicular plasma flows. Even although these fast magnetoacoustic waves have relatively small amplitudes and periods, prescribed by the parameters of the external loop, approaching the magnetic null point of the flaring site, they rapidly grow up because the decrease in the fast magnetoacoustic speed near the magnetic null point, and also because the effect of focusing by refraction (McLaughlin and Hood 2004). The fast magnetoacoustic wave evolution in the vicinity of an X-point is accompanied by the dramatic increase in the density of the periodically varying electric current induced by the wave. When the current density becomes greater than some threshold value $j_{\text {thres }}$, various micro-instabilities are excited in the plasma in the vicinity of the X-point. The micro-instabilities lead to the onset of micro-turbulence that can dramatically increase the transport coefficients in the plasma, in particular, producing "anomalous" resistivity. The resistivity $\eta$ then can be modelled by the piecewise relation,

$$
\eta= \begin{cases}\eta_{\text {class }}, & \text { for }|j| \leq j_{\text {thres }} \\ \eta_{\text {anom }}, & \text { for }|j|>j_{\text {thres }}\end{cases}
$$

where $\eta_{\text {class }}$ and $\eta_{\text {anom }} \gg \eta_{\text {class }}$ are the classical and anomalous values of the resistivity, respectively. Oscillatory behaviour of the anomalous resistivity causes periodic triggering of the magnetic reconnection that results, in turn, in periodic acceleration of charged particles. 

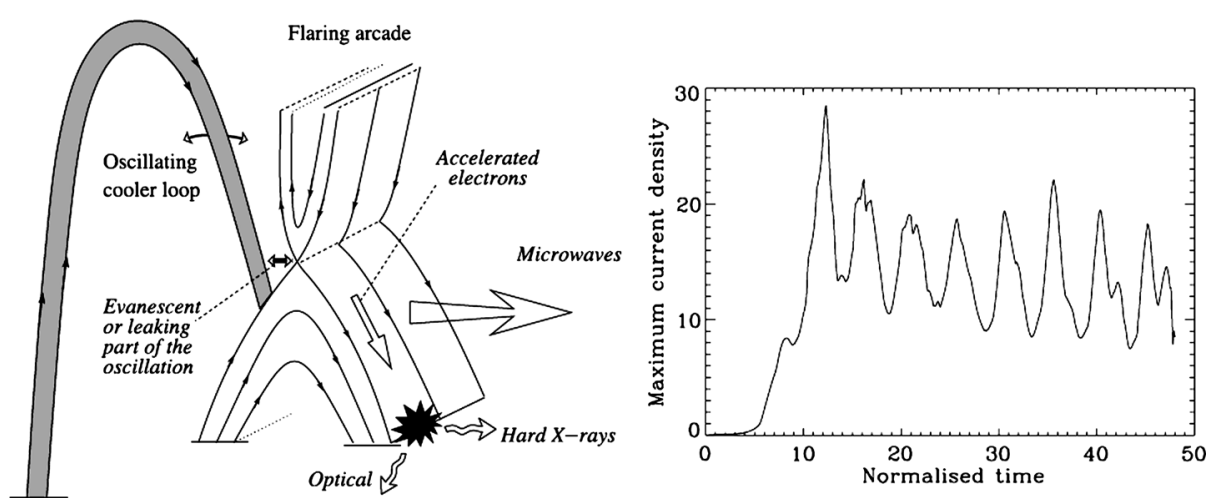

Fig. 44 Left panel: Sketch of the mechanism for the generation of flaring QPPs by an external magnetoacoustic oscillation. The cool (shaded) loop experiences transverse oscillations. A segment of the oscillating loop is situated near the flaring arcade. An external evanescent or leaking part of the oscillation can reach magnetic X-points or current sheets in the arcade, inducing quasi-periodic modulations of the electric current density. The resulting current driven plasma micro-instabilities are known to cause anomalous resistivity, which triggers periodic magnetic reconnection. Right panel: Time evolution of the maximum electric current density in the vicinity of a 2D magnetic neutral point. The current is generated by a harmonic fast magnetoacoustic wave with the period 5 time units, coming towards the neutral point. The initial relative amplitude of the wave is $1.5 \%$. The plasma $\beta$ is finite. The normalising values are the magnetic field strength and the Alfvén speed at the domain boundary, and the characteristic spatial scale of the field inhomogeneity. From Nakariakov et al. (2006)

The periodic variation of the density, energy and pitch angle distribution of non-thermal electrons produces QPPs of hard X-ray, gamma-ray, microwave, and other emissions. The period of these QPPs is prescribed by the external magnetoacoustic oscillation, and hence is not determined by the geometrical sizes of the flaring region.

A similar mechanism, but associated with slow magnetoacoustic waves, was independently developed by Chen and Priest (2006). The periodic modulation was proposed to be based on the variation of the plasma density in the wave in the vicinity of the reconnection site. Assuming that the electric current density in the current sheet in the reconnection site remains constant and changes much slower than the QPP period, the density variation in a slow wave leads to variation of the electron drift speed. When this drift speed overcomes some threshold speed, e.g. the ion-acoustic or even electron thermal speeds, ion-acoustic or Langmuir micro-turbulences are excited due to the ion-acoustic or Buneman instabilities. In the current-carrying plasma the micro-turbulence is known to be a cause of the anomalous electric resistivity.

The idea that flaring QPPs could be triggered by external MHD waves is also consistent with the observed progression of the flaring QPPs along the magnetic neutral line that separated the ribbons in large, "two-ribbon" solar flares (Nakariakov and Zimovets 2011). The impulsive energy releases of the hard X-ray and visible light emission on the solar surface are usually observed to propagate along the magnetic neutral line at the speed of a few tens of $\mathrm{km} \mathrm{s}^{-1}$. This value of the speed is significantly below the Alfvén and sound speeds in the corona. It can be interpreted in terms of slow magnetoacoustic waves guided by the plasma arcade that forms the flaring active region in the direction perpendicular to the magnetic field (see the discussion in Sect. 6.3). In a uniform medium slow magnetoacoustic waves are able to propagate either strictly along the magnetic field lines or weakly oblique, in a rather narrow cone along the field. However, in the presence of plasma non-uniformities these oblique slow waves can bounce between two reflecting or refracting boundaries positioned across 
the field, e.g. the footpoints of the coronal magnetic field lines, and hence move gradually in the direction perpendicular to the field.

In this scenario, a slow magnetoacoustic perturbation excited by an initial energy release somewhere at the top of the arcade propagates obliquely towards the arcade footpoints. There it experiences reflection on the sharp gradient of the sound speed and returns back to the top the arcade. This results into a slow motion of the wave along the arcade axis, across the field. The angle of the most efficient perpendicular propagation is about $25^{\circ}-28^{\circ}$, which corresponds to the perpendicular wave vector defined by the width of the wave guide and the distance between the footpoints along the magnetic field in the arcade. Hence, the wave arrives at the top of the arcade at the location shifted along the arcade's axis, because of the obliqueness of the wave vector. Approaching the X-point above the arcade, the slow wave triggers another act of the energy release-another burst of the QPPs. Moreover, the induced energy release reinforces the slow perturbation, compensating its dissipative and scattering losses. The period of the generated QPPs is similar to the second harmonics of the longitudinal mode of a coronal loop that is determined by the speed of the triggering slow magnetoacoustic wave and the travel path, i.e the loop length (Sect. 6.3). For typical parameters of solar flares the period of the induced QPPs is about 10-600 s, which is consistent with observations.

In addition, the discussed scenario can successfully address another frequently appearing interesting feature of flaring QPPs, the presence of double maxima in the elementary bursts (e.g. Zimovets and Struminsky 2009). According to the discussed model the double peaks of the emission may occur due to some asymmetry in the location of the wave source or in the arcade geometry. In this case, the slow pulses, reflected from the opposite footpoints, arrive at the arcade top and trigger the next elementary bursts at slightly different times and locations.

\subsection{Spontaneous Mechanisms for QPPs}

All described above mechanisms for QPPs generation are essentially based upon the modification of the flaring plasma by MHD waves. However a nonlinear process of magnetic reconnection that drives the flare, may occur in a spontaneous oscillatory regime. Such oscillatory or rather repetitive manner of reconnection can be seen as an effective "magnetic dripping". In this model the free magnetic energy is gradually accumulated and then, when some threshold value is achieved, rapidly released.

One possible mechanism consistent with this model is instability of a current sheet. Any sufficiently long and narrow current sheet is unstable owing to the tearing mode instability. As a result of this instability, spatially periodic O-type magnetic islands or plasmoids in 2D description, or spatially periodic magnetic ropes (current-carrying loops) with the helical magnetic field structures in 3D description (in both cases they are called a "plasmoid chain") are formed. The spatial periodicity can then result in time periodicity. For example, Loureiro et al. (2007) have checked the Sweet-Parker solution of magnetic reconnection in a simple current sheet. Introducing an additional small parameter of the problem $\epsilon=\delta_{\mathrm{cs}} / L_{\mathrm{cs}}$, where $\delta_{\mathrm{cs}}$ and $L_{\mathrm{cs}}$ are the width and length of the current sheet, they found a new solution unstable due to the plasmoid chain instability. The maximum growth rate of this instability depends on $\epsilon$ as $\gamma_{\text {plas }} \sim \epsilon^{-1 / 2}$.

A spontaneous oscillatory regime was seen in the numerical simulation of magnetic reconnection in a large-scale 2D current sheet, located above the flaring loop in the standard model of a flare (Kliem et al. 2000). Such a current sheet is the key element of the standard model of a solar flare, see Sect. 11.1. The simulated reconnection was caused by the 
Fig. 45 Upper: Electric field at the main X-points (reconnection rate) vs. time. Bottom: Location of the dominant X-point.

Distances and time are expressed in a halfwidth of the current sheet and Alfvén cross time, respectively. From Kliem et al. (2000)

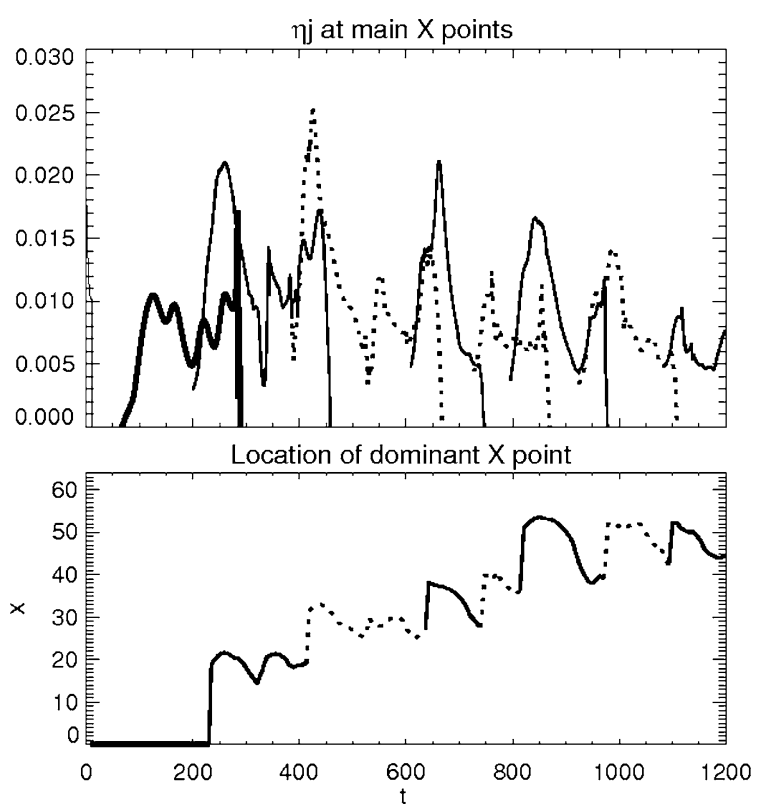

anomalous resistivity, which was a self-consistent parameter in the model, and defined by the electron-ion drift velocity, proportional to the electric current density (see Eq. (71)). The repetitive regime was found to appear because the coalescence of continuously generated plasmoids, that resulted in formation of one or several hot and dense larger plasmoids. When finally such a plasmoid becomes sufficiently large it is rapidly ejected along the current sheet upwards or downwards. Usually a plasmoid is ejected at about the Alfvén speed (e.g. Shibata and Magara 2011), that enables new magnetic flux to continuously enter the current sheet, reinforcing the reconnection. However if the plasma is unable to carry a sufficient amount of the magnetic flux into the diffusion region to support the Alfvénic outflow in the steady Petschek state, the system keeps switching between the fast Petschek and slow Sweet-Parker regimes of reconnection. Such repetitive ejection of plasmoids from the vertical current sheet above in the flaring site (Fig. 45) was found to be accompanied with the production of quasi-periodic bursts of non-thermal electrons accelerated at the X-points separating the plasmoids (Karlický et al. 2010). The non-thermal electrons produce QPPs of all kinds of the emission. The period of QPPs, predicted by the model, is determined by the plasma concentration $n_{0}$ and temperature $T_{0}$ inside the current sheet, and the magnetic field $B_{0}$ outside it,

$$
P_{\text {plasmoid }} \propto n_{0}^{-1 / 2} T_{0}^{2} B_{0}^{-1} .
$$

For the plasma parameters typical for flaring active regions, the time between pulsations is 0.4-20 s, which is consistent with observations. This model is rather suitable for QPPs with partly irregular intervals between the peaks, as the plasmoid shedding is a fairly irregular process.

The abovementioned pulsations appear when in the flare current sheet there are dominant and large plasmoids. But generally, in the current sheet, due to the stretching, tearing and merging processes, a cascade of plasmoids with a broad range of spatial sizes can be formed (Bárta et al. 2011; Karlický and Bárta 2011). In this case, the reconnection is in the turbulent regime and numerical results and observations reveal not only pulsations with 
some characteristic periods, but also turbulence, which Fourier spectra have a power-law form (see, e.g., Karlický et al. 2005; Bárta et al. 2011).

The merging of plasmoids can also cause QPPs. Tajima et al. (1987) found numerically and analytically that during the merging (coalescence) of two current-carrying (twisted) loops the resulting loop oscillated with the period

$$
P_{\text {coales }} \approx l / V_{\mathrm{A}},
$$

where $l$ is the characteristic size of the process, e.g. the minor radius of the loop, and $V_{\mathrm{A}}$ is the characteristic Alfvén speed, giving us a value of about several seconds. These oscillations are accompanied by quasi-periodic acceleration of charged particles, and hence can produce QPPs of thermal and non-thermal emission (e.g. Karlický and Kliem 2010; Karlický and Bárta 2011).

An entirely different mechanism for periodic processes during solar flares comes from the so-called cyclic magnetic reconnection (Karlický 2009). Namely, if somewhere there is a rotating plasma (vortex) in the high- $\beta$ regime, this rotating plasma can amplify the magnetic field by the dynamo process. Due to the non-zero electrical resistivity the stressed magnetic field can repeatedly reconnect at locations close to a boundary of the rotating plasma and release the magnetic energy in periodic way. If it is associated with an efficient electron acceleration then these accelerated electrons can generate a periodically varying emission.

Spontaneous production of QPPs could also be initiated by an external nonlinear fast magnetoacoustic pulse. For example, McLaughlin et al. (2012) found in the 2D X-point geometry, that an incoming fast oblique magnetoacoustic shock wave excited by some external driver, refracts towards the $\mathrm{X}$-point, and significantly deforms the magnetic X-point into a cusp-like point which in turn collapses to a current sheet. The system then evolves periodically through a series of horizontal and vertical current sheets with associated changes in magnetic connectivity. The oscillation period was found to be inversely proportional to the amplitude of the triggering fast wave, indicating the nonlinear nature of the oscillation. The oscillations decay exponentially with the decay time comparable with the oscillation period. For typical coronal conditions the periods ranged in the vicinity of one minute, which is consistent with observed values of QPPs.

Despite significant progress in understanding of MHD self-oscillations and flaring QPPs in general, spontaneous and induced magnetic dripping mechanisms deserve a systematic study both theoretically and in observations. Important issues that one needs to address are the following:

- Is it possible to construct a low-dimensional semi-empirical model that would link physical conditions in the reconnection site with the period of pulsations?

- Can the multi-periodic QPPs, occasionally detected in observations be explained by this mechanism, or they solidly evidence in favour of MHD-wave-based mechanisms for QPPs? Indeed, the presence of several spectral peaks can be readily attributed to the excitation of different MHD modes and their different spatial harmonics in the flaring active region.

- Do the macroscopic resonators, such as, e.g. the fast magnetoacoustic resonator formed between the plasma cusp and the solar surface in the standard flare model, play any role in the periodicity? A similar question is applicable to the longitudinal and torsional Alfvénic waves that bounce between the cusp region and the footpoints of the magnetic field lines.

- What determines whether the effect of plasmoid shedding is of quasi-periodic or turbulent (with the power-law statistics of the shedding) character?

Possible geophysical implications of flaring QPPs are discussed in Sect. 13. 
A useful piece of information can be obtained from the magnetospheric manifestation of quasi-periodic regimes of reconnection, in sub-storms. In particular, pulsed reconnection in the magnetotail was shown to be connected with Pi2 in Keiling et al. (2006). It was established that a series of 1-min-long nightside flux transfer event (NFTE) pulses following each other within 1-3 min, resembled the magnetic field signature of Pi2. NFTEs are believed to form during the reconfiguration of tail magnetic field lines, i.e., reconnection. It was concluded that the quasi-periodic reconnection was both the energy provider and the frequency driver for the Pi2 registered on the ground and also in space. Quasi-periodic magnetic reconnection in the magnetotail can also be responsible for the quasi-periodicity in the bursty bulk flow (BBF) events that are about-10-min timescale variable flows observed in the near-Earth and mid-tail plasma sheet.

\section{Beyond MHD}

Modern magnetospheric observations with a high temporal-spatial resolution during various geophysical processes indicate that a larger power density tends to be concentrated in smaller time and space scales. Thus, impulsive and small-scale processes, despite their relatively short duration and small scale, may involve substantial energy exchange between the media. As an example of impulsive energy exchange in the coupled system magnetosphereionosphere-atmosphere one may recall the bursty process with a largest energy release in the terrestrial space plasma - the magnetospheric substorm. The mechanism of the substorm onset has not been identified yet, but probably it is related to small-scale non-MHD processes. For example, kinetic small-scale Alfvénic structures can carry a very localised, but intense, field-aligned electric field, resulting in a downward acceleration of auroral electrons (Chaston et al. 2002a). Likewise, understanding important processes in solar flares, e.g. charged particle acceleration and onset of micro-turbulence, requires employment of micro-physics. For example, the vital role of the anomalous resistivity has already been highlighted above (Sect. 11.4 and 11.5).

\subsection{Dispersive Alfvén Waves in the Magnetosphere}

For small scale processes, the dispersion relation for shear Alfvén waves, $\omega=\omega_{\mathrm{A}} \equiv k_{z} V_{\mathrm{A}}$, is modified:

- in the kinetic limit, to $\omega=\omega_{\mathrm{A}} \sqrt{1+\left(k_{\perp} \rho_{\mathrm{i}}\right)^{2}}$, where $\rho_{\mathrm{i}}$ is the Larmor ion radius;

- in the inertial limit, to $\omega=\omega_{\mathrm{A}} / \sqrt{1+\left(k_{\perp} \lambda_{\mathrm{e}}\right)^{2}}$, where $\lambda_{\mathrm{e}}=c / \omega_{\mathrm{pe}}$ is the electron inertial length (the collisionless plasma skin depth),

and thus the wave becomes dispersive even in a uniform medium. We should stress that these mechanisms for the MHD wave dispersion are different from the low-frequency geometrical dispersion caused by the waveguiding effect in the presence of a plasma or magnetic field non-uniformity, discussed in Sect. 4.

When dispersive effects are not large, $\left(\lambda_{\mathrm{e}}, \rho_{\mathrm{i}}\right) \ll k_{\perp}^{-1}$, these dispersion relationships can be combined into one $\omega \simeq \omega_{\mathrm{A}} \sqrt{1 \pm\left(k_{\perp} \rho_{\mathrm{d}}\right)^{2}}$, where $\rho_{\mathrm{d}}^{2} \simeq \rho_{\mathrm{i}}^{2}+\lambda_{\mathrm{e}}^{2}$ is the dispersion radius. The actual influence of dispersive effects is determined by the structure parameter $\left\langle\rho_{\mathrm{d}}\right\rangle$ which is an integral along field lines (Leonovich and Mazur 1995a).

In contrast to waves in ideal MHD approximation, small-scale dispersive Alfvénic structures with scales comparable with the dispersive radius $\rho_{\mathrm{d}}$, possesses intrinsic field-aligned 
electric field $E_{\|}$,

$$
\left|E_{\|}\right|=\left(k_{\perp} \rho_{\mathrm{d}}\right)^{2}\left(k_{\|} / k_{\perp}\right)\left|E_{\perp}\right| .
$$

Due to this field, dispersive Alfvén waves can effectively accelerate and precipitate electrons, which is especially important for the physics of the auroral regions (Borovsky 1993; Stasiewicz et al. 2000) and may be relevant to solar flares. Dispersive Alfvén waves can be driven by compressive magnetoacoustic waves via the mode conversion (Klimushkin and Mager 2014).

In an inhomogeneous system, a shear Alfvén wave tends to evolve into small-scale dispersive Alfvén wave due to the process of phase mixing. The transverse spatial scale of an Alfvén wave in an inhomogeneous plasma increases with time as $k_{\perp}(t)=\partial_{x} \omega_{\mathrm{A}}(x) t$. This effect is simply connected to the intrinsic property of Alfvén waves that are situated at individual magnetic surfaces of constant Alfvén speed perturbing the field and displacing the plasma along those surfaces. Thus Alfvén waves situated at neighbouring magnetic surfaces "do not feel" each other, and can quickly become out of phase. This produces secularly growing transverse gradients, and hence a perpendicular energy cascade to smaller and smaller transverse scales, until the high-frequency dispersion effects come into play. In the saturation state, the typical transverse scale of oscillations in the dispersive Alfvén resonator formed in the magnetosphere between the conjugate ionospheres is $\delta_{\mathrm{d}}=\left(a \rho_{\mathrm{d}}^{2}\right)^{1 / 3}$. This scale, $k_{\perp} \simeq\left(a \rho_{\mathrm{d}}^{2}\right)^{-1 / 3}$, is reached after an elapsed time $\tau_{\mathrm{d}}=\omega_{\mathrm{A}}^{-1}\left(a / \rho_{\mathrm{d}}\right)^{2 / 3}$. Estimates show that at auroral latitudes $\left\langle\rho_{\mathrm{d}}\right\rangle$ varies from a few $\mathrm{km}$ to a few tens of $\mathrm{km}$. For $a=400 \mathrm{~km}$ and $\left\langle\rho_{\mathrm{d}}\right\rangle=1-10 \mathrm{~km}$ the transverse scale $\delta_{\mathrm{d}}$ determined by dispersive effects is $7-15 \mathrm{~km}$.

\subsection{Field-Aligned Potential Drop, Turbulent Layer, and Alfvén Waves}

In a collisionless warm (finite- $\beta$ ) plasma a substantial non-resistive potential drop can be produced by mirror forces. The field-aligned electric field $E_{\|}$is supported by the difference in pitch angle distributions for electrons and ions under conditions of plasma quasineutrality. Using stationary distribution functions consistent with a distribution of potential $\phi(s)$ the field-aligned current $j_{\|}$can be calculated. A total potential difference $\Delta \phi=\phi_{\mathrm{m}}-\phi_{\mathrm{i}}$ along a field line, e.g. between the equatorial magnetosphere and ionosphere could be as large as $e \phi \simeq E\left(B_{\mathrm{m}} / B_{\mathrm{i}}\right)$, where $E$ is the particle energy, and $B_{\mathrm{m}} / B_{\mathrm{i}}$ is the mirror ratio, and the subscripts $\mathrm{m}$ and $\mathrm{i}$ indicate the magnetosphere and ionosphere, respectively. This potential drop is necessary to support the field-aligned current in a magnetic trap with a small cone angle, through which current-transporting electrons can reach the bottom ionosphere. In general, $j_{\|}$is a functional of $\phi(s)$, i.e. $j_{\|}=j(\phi(s))$. Kinetic consideration shows that an Ohm's-type law may exist for the upward current in a mirror-confined plasma for a certain range of potential values. The current-voltage relationship between $j_{\|}$and the potential drop can be presented in non-local forms,

$$
\Delta \phi=Q j_{\|}, \quad \text { or } \quad j_{\|}=K \Delta \phi,
$$

where $Q$ is the effective electrical resistance. The field-aligned conductance $K=Q^{-1}$ of a flux tube can be estimated as $K \simeq n e^{2} / m_{\mathrm{e}} u_{\mathrm{e}}$, where $m_{\mathrm{e}}, n$, and $u_{\mathrm{e}}$ are the mass, concentration, and thermal speed of magnetospheric electrons, respectively (Borovsky 1993). Even this simplified linear current-voltage relationship fits observations rather well. The mirror force produces an effective electric resistance, which may be coined a mirror resistance, accompanied by a dissipation of energy. It is natural to expect that dissipation processes in 
Fig. 46 Schematic illustration of the Alfvén wave interaction with the auroral upper ionosphere, comprising the auroral acceleration region (AAR)

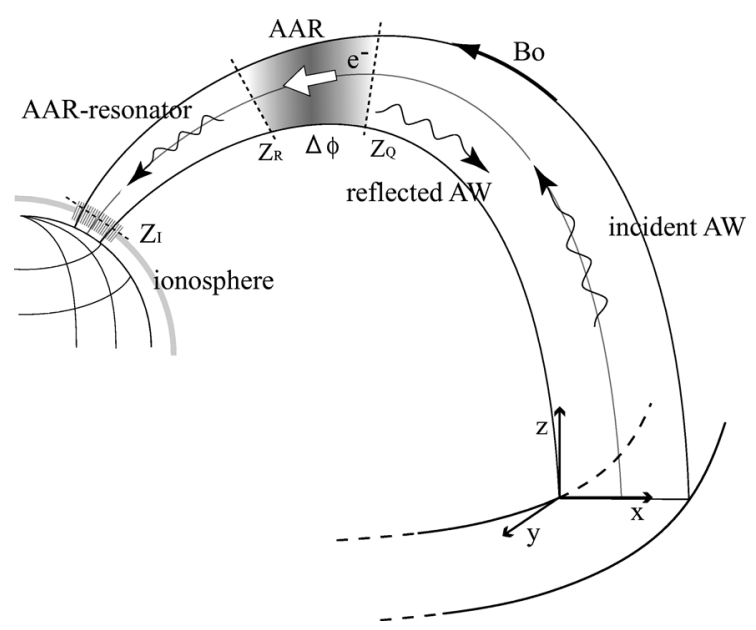

a mirror-confined plasma with an effective field-aligned electric resistance could influence the dissipative properties of Alfvén waves in such system (Lysak and Dum 1983a).

In the magnetosphere, this potential drop is localised in a thin sheet, named the auroral acceleration region (AAR, see Fig. 46). Given a prescribed potential drop, the dissipated energy of the Alfvén waves upon transmission through the AAR can be estimated. In the linear regime the potential drop through the AAR is related to the field-aligned current by a linear voltage-current relationship given by Eq. (75). The AAR for MHD disturbances is actually a thin film with a negligible transverse conductivity, therefore the currents $j_{\|}$at the magnetospheric and ionospheric sides of the AAR are equal, and the wave electric potential is continuous through the AAR. These assumptions lead to the general expression for the coefficient of an Alfvén wave reflection from the combined AAR-ionosphere system,

$$
R\left(k_{\perp}\right)=\frac{S_{\mathrm{P}}\left(k_{\perp}\right)-\Sigma_{\mathrm{A}}}{S_{\mathrm{P}}\left(k_{\perp}\right)+\Sigma_{\mathrm{A}}}, \quad S_{\mathrm{P}}\left(k_{\perp}\right)=\frac{\Sigma_{\mathrm{P}}}{1+Q \Sigma_{\mathrm{P}} k_{\perp}^{2}} .
$$

Thus, we can account for the AAR by just replacing in the boundary conditions given by Eq. (58) the Pedersen ionospheric conductivity $\Sigma_{\mathrm{P}}$ with the modified conductivity $S_{\mathrm{P}}\left(k_{\perp}\right)$, dependent on the transverse component of the wave vector $k_{\perp}$ (Vogt 2002). Therefore all the basic results for coupling of magnetospheric Alfvén waves with the combined AARionosphere system can be obtained by this substitution.

Two spatial scale parameters can be introduced, the resistive scale length of magnetosphere-ionosphere coupling $\lambda_{\mathrm{P}}=\sqrt{Q \Sigma_{\mathrm{P}}}$, and the wave transit scale $\lambda_{\mathrm{A}}=\sqrt{Q \Sigma_{\mathrm{A}}}$. When $k_{\perp}^{-1}>\lambda_{\mathrm{P}}$, the Alfvén wave reflection is determined mainly by the dimensionless ionospheric Pedersen conductivity $\Sigma_{\mathrm{P}} / \Sigma_{\mathrm{A}}$. The AAR begins to control the reflection of Alfven disturbances when their transverse scales become comparable to the resistive scale length of magnetosphere-ionosphere coupling, $\lambda_{\mathrm{P}}$. When $k_{\perp}^{-1} \leq \lambda_{\mathrm{A}}$, the reflection properties of the combined AAR-ionosphere system changes dramatically, and a substantial part of incident wave energy can be absorbed inside the ARR. This energy does not dissipate locally, but is transported from the collisionless plasma into the ionosphere.

It is important to estimate the damping rate (decrement) $\gamma_{n}$ and the width of the resonance region $\delta_{\mathrm{n}}$ of the field line Alfvén resonator under the combined action of the ionospheric Joule dissipation and the magnetospheric field-aligned potential drop. A specific scale $l_{\mathrm{A}}=$ $\left(\frac{2}{n \pi} a \lambda_{\mathrm{A}}^{2}\right)^{1 / 3}$, where $n$ is the Alfvén harmonic number and $a$ is the scale of inhomogeneity, 
which can be named the resistive Alfvén resonance scale, can be introduced. If the wave transit scale $\lambda_{\mathrm{A}}$ is relatively small, so $\lambda_{\mathrm{P}} \ll l_{\mathrm{A}}$, the width of the Alfvén resonance and the quality factor $Q_{\mathrm{n}}^{(\mathrm{A})}$ are determined mainly by the ionospheric conductivity,

$$
\delta_{\mathrm{n}} \simeq \frac{2 a}{n \pi} \frac{\Sigma_{\mathrm{A}}}{\Sigma_{\mathrm{P}}}, \quad Q_{\mathrm{n}}^{(\mathrm{A})}=\frac{2 \delta_{\mathrm{n}}}{a}=\frac{\pi n}{4}\left(\frac{\Sigma_{\mathrm{P}}}{\Sigma_{\mathrm{A}}}\right) .
$$

In the opposite case of a large scale of $\lambda_{\mathrm{A}}$, when $\lambda_{\mathrm{P}} \gg \lambda_{\mathrm{A}}$, the energy losses in the AAR due to the mirror resistance are the dominant damping mechanism of Alfvén resonant oscillations

$$
\delta_{\mathrm{n}} \simeq\left(\frac{2 a \lambda_{\mathrm{A}}^{2}}{n \pi}\right)^{1 / 3} \quad Q_{\mathrm{n}}^{(\mathrm{A})} \simeq\left(\frac{n \pi}{8} \frac{a^{2}}{\lambda_{\mathrm{A}}^{2}}\right)^{1 / 3} .
$$

It is important to note that the actual width of an Alfvén resonance with the AAR is given by Eq. (78) as $l_{\mathrm{A}} \simeq\left(a \lambda_{\mathrm{A}}^{2}\right)^{1 / 3}$, not by $\lambda_{\mathrm{A}}$.

Dynamic magnetospheric processes, such as substorms, yield a large energy flow from the plasma sheet and magnetotail into the auroral ionosphere. Polar satellite observations revealed that the Pointing flux of the electromagnetic wave energy along a magnetic flux tube conjugate to an auroral arc is much higher than the flux of the energetic electrons, and is more than sufficient (about 2 orders of magnitude) to drive the relevant auroral intensification (Wygant et al. 2000). Analysis of the long-term coordinated polar observations of the ultra-violet auroral emission and corresponding electromagnetic disturbances in the magnetosphere proved that a substantial (up to 1/3) part of the aurora intensifications is fed by the wave channel of the electromagnetic energy transfer into the auroral ionosphere, in the auroral electron acceleration region (Keiling et al. 2002). Perhaps this mechanism can be considered in conjunction with the role played by Alfvén (or, perhaps, kink) waves in the impulsive phase of a solar flare (Fletcher and Hudson 2008).

At auroral regions, a very effective reflection boundary for the intermediate-scale Alfvén waves, of transverse scale $\leq 10^{2} \mathrm{~km}$, can be formed at the lower edge of the auroral acceleration region located at altitudes about $1--2 R_{\mathrm{E}}$. In these regions a specific IAR can be formed with somewhat lower frequencies than that of a typical IAR (Pilipenko et al. 2002).

\subsection{Interaction of Alfvén Waves with a Turbulent Layer}

Alfvén waves play an important role in dynamic processes in space plasmas by transporting without geometric attenuation non-stationary field-aligned currents to considerable distances. Space, solar and astrophysical plasmas are often turbulent, so scattering of particles by turbulent noise becomes more efficient than Coulomb collisions. Such anomalous collisions of particles with turbulent noise result in the occurrence of finite anomalous plasma resistivity, discussed in Sect. 11.5 and 11.4 in the solar context, and formation of turbulent layers in a space plasma. Quite often, e.g. at auroral latitudes, high-frequency turbulence is confined in narrow regions where the threshold for current instability is minimal. The width of such a layer with anomalous resistivity might be small compared with the field-aligned scale of low-frequency Alfvén waves. Therefore, for the description of the wave interaction with a turbulent layer in this regime the thin layer approximation may be used. This approximation enables one to obtain simpler analytical relationships and to reveal the physical parameters that control this interaction.

The problem of Alfvén wave interaction with a turbulent layer has much in common with the problem of Alfvén wave transmission through a layer with a field-aligned potential drop. 
Fig. 47 Sketch illustrating the model of Alfvén wave interaction with a flat turbulent layer of width $h$ with the anomalous transverse $\sigma_{\perp}$ and field-aligned $\sigma_{\|}$conductivities

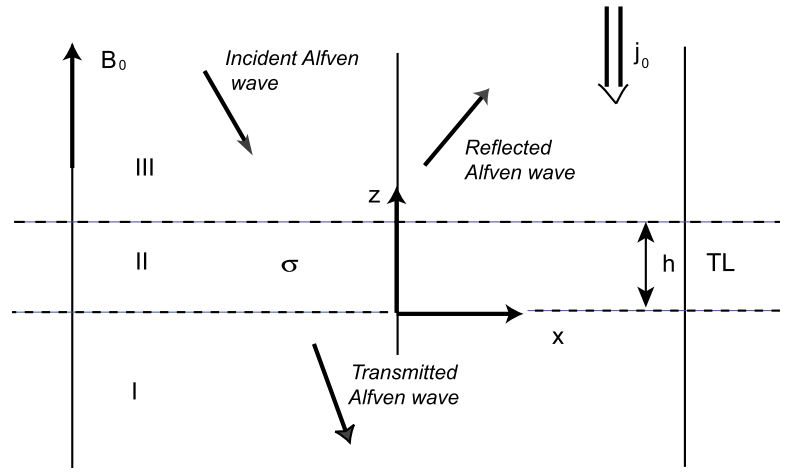

The former mechanism is caused by local resistivity due to anomalous collisions, whereas the latter mechanism is related to the non-resistive potential drop caused by mirror forces in a magnetic flux tube. The basic notions about Alfvén wave interaction with a turbulent layer with anomalous field-aligned resistance were formulated by Lysak and Dum (1983b), who showed that waves can partially penetrate the layer, be absorbed in it, and reflected from it. The relative rate of these processes turned out to be dependent on the transverse wave scale.

We consider interaction of Alfvén waves with a flat turbulent layer of width $h$ with the complex transverse $\sigma_{\perp}$ and field-aligned $\sigma_{\|}$conductivities. The real parts of these conductivities are caused by the occurrence of collective collisions of electrons and ions with turbulent noise. It is assumed that micro-turbulence is supported by external sources, and is not created by the Alfvén wave itself (Fig. 47).

From Ohm's law in a medium with the transverse $\sigma_{\perp}(\omega)$ and parallel (field-aligned) $\sigma_{\|}(\omega)$ complex conductivities, $\mathbf{j}_{\perp}=\sigma_{\perp} \mathbf{e}_{\perp}$, and $\mathbf{j}_{\|}=\sigma_{\|} \mathbf{e}_{\|}$, Maxwell's equations reduce to

$$
\partial_{z}\left(\begin{array}{l}
e_{x} \\
b_{y}
\end{array}\right)=\hat{\mathbf{T}}\left(\begin{array}{l}
e_{x} \\
b_{y}
\end{array}\right), \quad \hat{\mathbf{T}}=\left(\begin{array}{cc}
0 & i \omega-k_{\perp}^{2} / \mu_{0} \sigma_{\|} \\
-\mu_{0} \sigma_{\perp} & 0 .
\end{array}\right)
$$

In a resistive MHD medium with isotropic conductivity $\sigma_{\|}=\sigma_{\perp}$ the dispersion equation for Alfvén waves in a resistive medium by Tikhonchuk and Bychenkov (1995) is widely used. However, a turbulent medium is more adequately modelled as a three-component plasma: electrons, ions, and plasmons or vortices (neutral quasi-particles). The transverse conductivity of such a medium is composed of electron and ion conductivities. In the case of lowfrequency waves, $\omega \ll \Omega_{\mathrm{i}}$, a rather complicated expression for the conductivity tensor can be reduced to

$$
\sigma_{\perp} \simeq \sigma_{\mathrm{P}}-\frac{i \omega}{\mu_{0} V_{\mathrm{A}}^{2}}, \quad \sigma_{\|}^{-1} \simeq \mu_{0} \lambda_{\mathrm{e}}^{2}\left(v_{e}-i \omega\right) .
$$

The complex transverse conductivity $\sigma_{\perp}$ is composed of the contributions from both the static Pedersen conductivity $\sigma_{\mathrm{P}}$ and polarisation current.

The generalised dispersion equation for Alfvén waves in a turbulent medium has the form

$$
k_{\|}^{2}=\left(1+k_{\perp}^{2} \lambda_{\mathrm{e}}^{2}\right)\left(k_{\mathrm{A}}^{2}+i \omega \mu_{0} \sigma_{\mathrm{P}}\right)-\frac{\sigma_{\mathrm{P}}}{\sigma_{0}} k_{\perp}^{2}+i \frac{k_{\mathrm{A}}^{2} k_{\perp}^{2}}{\omega \mu_{0} \sigma_{0}},
$$

where $\lambda_{\mathrm{e}}=c / \omega_{\mathrm{pe}}$ is the plasma inertial scale. The static field-aligned conductivity $\sigma_{0}$ is provided by electron collisions, whereas the Pedersen conductivity is supported by electron- 
ion collisions. The effective skin-depths, $\delta_{\|}$and $\delta_{\mathrm{P}}$, determined by the field-aligned and transverse conductivities, correspondingly, can be introduced as

$$
\delta_{\|}=\left(1+k_{\perp}^{2} \lambda_{\mathrm{e}}^{2}\right)^{-1 / 2} \sqrt{\frac{2}{\omega \mu_{0} \sigma_{0}}}, \quad \delta_{\mathrm{P}}=\sqrt{\frac{2}{\omega \mu_{0} \sigma_{\mathrm{P}}}} .
$$

The damping spatial rate $\left(k_{\|}=k_{\mathrm{A}}+i \kappa\right)$ of the Alfvén wave decay in a turbulent medium can be estimated from the generalised dispersion equation (81) that comprises both field-aligned and transverse resistances

$$
\frac{\kappa}{k_{\mathrm{A}}} \simeq \frac{1}{4}\left(k_{\perp} \delta_{\|} l\right)^{2}+\left(k_{\mathrm{A}} \delta_{\mathrm{P}}\right)^{-2} .
$$

The reflection and transmission coefficients of an Alfvén wave upon interaction with the multi-layer turbulent layer-ionosphere system can be calculated using the method of effective impedances (Fedorov et al. 2007). Knowledge of those coefficients enables us to estimate the energy loss $J$ of Alfvén waves in a turbulent layer.

One can introduce the dissipative scale $\lambda_{\mathrm{P}}=\sqrt{\left|Q \Sigma_{\perp}\right|}$, where $Q=h / \sigma_{0}$ is the fieldaligned integrated resistance of the layer. The parameter $\lambda_{\mathrm{A}}=\sqrt{Q \Sigma_{\mathrm{A}}}$ is determined by the altitude-integrated field-aligned resistance $Q$ and the Alfvén wave conductance $\Sigma_{\mathrm{A}}=$ $\left(\mu_{0} V_{\mathrm{A}}\right)^{-1}$ above the turbulent layer. According to its physical sense, this parameter is the transverse scale when the field-aligned column resistance matches the Alfvén wave resistance $\Sigma_{\mathrm{A}}^{-1}$. The previously introduced parameter, the resistive length $\lambda_{\mathrm{P}}$, is the transverse wave scale when the scale of wave penetration into a turbulent layer with conductivity $\sigma_{\|}$ matches the layer width $h$. As one will see further, the key parameter of the Alfvén wave interaction with a thin turbulent layer is the ratio between $\lambda_{\mathrm{A}}$ and the wave transverse scale, namely $k_{\perp} \lambda_{\mathrm{A}}$. At the same time, the ratio between the parameter $\lambda_{\mathrm{P}}$ and $k_{\perp}^{-1}$ more adequately characterises the wave dissipation in a thin turbulent layer.

As an example, the interaction of an Alfvén wave with the period of $100 \mathrm{~s}$ with a turbulent layer has been calculated using models with different types of turbulence. The parameters of the background plasma have been chosen to correspond to typical values in the auroral cavity of the upper ionosphere for two model layers:

Model TF (Trakhtengertz and Feldstein 1985): $v_{\mathrm{e}}=10^{2} \mathrm{~s}^{-1}, v_{\mathrm{i}}=\sqrt{m_{\mathrm{e}} / m_{\mathrm{i}}} v_{\mathrm{e}}=2.3 \mathrm{~s}^{-1}$, the anomalous field-aligned conductivity $\sigma_{0}=2.8 \times 10^{-1} \Omega^{-1} \cdot \mathrm{m}^{-1}$, resistance $Q=h / \sigma_{\|} \simeq$ $0.35 \times 10^{6} \Omega \cdot \mathrm{m}^{2}$, Pedersen conductivity $\sigma_{\mathrm{P}}=0.19 \cdot 10^{-5} \Omega^{-1} \cdot \mathrm{m}^{-1}$, Pedersen conductance $\Sigma_{\mathrm{P}}=0.19 \Omega^{-1}$, the Alfvén resistive scale $\lambda_{\mathrm{A}} \simeq 0.5 \mathrm{~km}$, and the dissipative scale $\lambda_{\mathrm{P}} \simeq$ $0.26 \mathrm{~km}$.

Model S: A layer with ion-acoustic turbulence. Anomalous collision frequencies are estimated with the use of weak turbulence theory. A feature of this model is a strong contrast $\left(\sim 4 \times 10^{4}\right)$ between the electron $v_{\mathrm{e}}$ and ion $v_{\mathrm{i}}$ collision frequencies.

The dependence of the energy loss $\alpha=J / S^{(i)}$, normalised to the energy flux of the incident waves $S^{(i)}$, in a turbulent layer with anomalous conductivity, on the transverse wave number for models TF and $\mathrm{S}$ is shown in Fig. 48. For both turbulent layer models, the energy losses are maximal at wave scales $k_{\perp} \sim \lambda_{\mathrm{A}}^{-1}$. The part of the energy loss, $\alpha_{\perp}\left(k_{\perp} \lambda_{\mathrm{A}}\right)$ associated with the transverse resistance has a weak dependence on the wave scale. In contrast, the part $\alpha_{\|}\left(k_{\perp} \lambda_{\mathrm{A}}\right)$ reaches the peak values at $k_{\perp} \lambda_{\mathrm{A}} \simeq 1.4$. This peak is responsible for the occurrence of a maximum in the dependence of the total energy losses on the wave number $\alpha\left(k_{\perp} \lambda_{\mathrm{A}}\right)$.

Thus, Alfvén waves impinging on a turbulent layer with anomalous resistivity can partially reflect from it, be absorbed, and be transmitted through it. When the field-aligned resistivity dominates, the relative effectiveness of these processes strongly depends on the wave 
Fig. 48 Dependence of the energy losses in an Alfvén wave interacting with a finite-width turbulent layer of thickness $h$ with anomalous conductivity, on the transverse wave number for models TF and S. The energy losses are normalised to the energy flux of the incident wave. The energy losses due to field-aligned $\alpha_{\|}$and transverse $\alpha_{\perp}$ conductivities, as well as the total energy losses $\alpha$, are shown separately
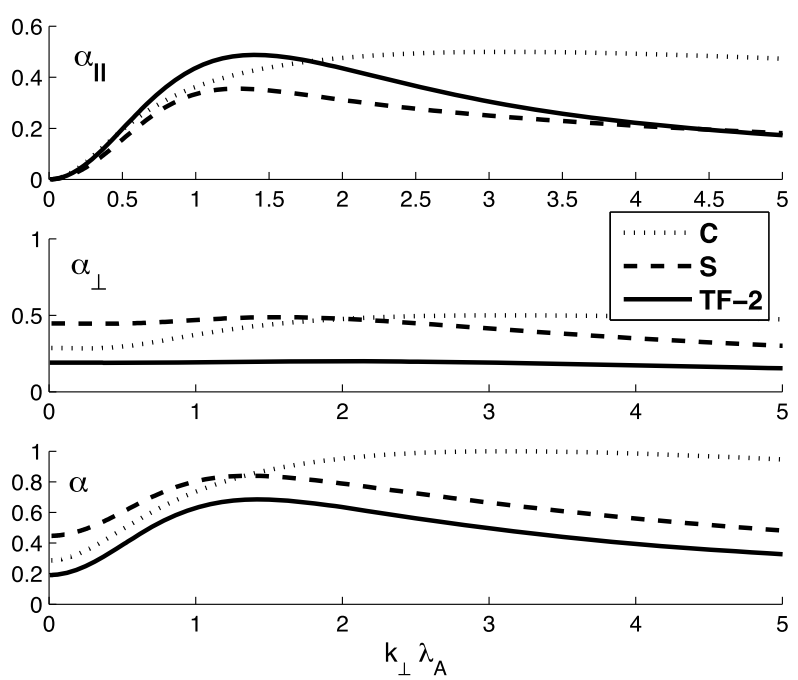

transverse scale. A turbulent layer totally screens small-scale disturbances, and only weakly absorbs large-scale disturbances. Wave energy losses are determined by the transverse resistance for large-scale disturbances, $k_{\perp} \lambda_{\mathrm{A}} \ll 1$. For small-scale disturbances $k_{\perp} \lambda_{\mathrm{A}} \gg 1$ both mechanisms contribute at a similar rate. For intermediate wave scales, $k_{\perp} \sim \lambda_{\mathrm{A}}^{-1}$, the energy losses are mostly determined by the field-aligned resistance. Thus, the occurrence of even a thin turbulent layer with anomalous conductivity results in a substantial scale-dependent damping rate of propagating Alfvén waves.

Similar turbulent layers can appear in the vicinity of solar flares, during turbalisation of various coronal jets, in association with CMEs, etc. The effect of coronal plasma turbulence on the propagation of MHD waves should also be investigated.

\subsection{Kinetic Description of Compressive ULF Modes}

Small-scale compressive (the high- $m$ Alfvén waves in a hot plasma with curved magnetic field lines) ULF waves are subject to intensive interaction with plasma particles, and hence demand a special treatment beyond standard MHD.

\subsubsection{Slow Modes}

Some compressive ULF pulsations could be associated with slow magnetoacoustic waves (Leonovich et al. 2006; Du et al. 2011), similar to those that are abundant in the solar corona (Sect. 6.3). However, the diamagnetic property of these waves cannot be considered as confirmative observational evidence. While the slow mode is a natural solution to the MHD equations, the very applicability of the MHD approximation to a collisionless plasma is questionable. Indeed, the condition for the slow mode is the transfer of momentum along field lines. In dense plasmas (like the main part of the low solar corona), momentum transfer is provided by particle collisions. Since the magnetospheric plasma is almost collisionless, this mechanism is ineffective.

Let us consider how the slow mode is derived in a collisionless plasma. To obtain the slow mode dispersion relation, ions with concentration $n_{\mathrm{i}}$ must be treated as particles with thermal velocities much lower than the parallel phase speed of the wave. On the other hand, 
electrons must be treated as particles with thermal velocities much higher than the wave phase speed. Additionally, let us assume that the plasma also contains cold electrons $n_{\mathrm{c}}$. If the cold electron number density is much smaller than the hot electron number density then we have that $n_{\mathrm{c}} \ll n_{\mathrm{h}}$. The electromagnetic field of the compressive wave under such conditions can then be described by two variables: the longitudinal magnetic field of the wave $b_{\|}$and the "parallel potential" $\varphi_{\|}$related to the parallel electric field of the wave. In these variables, the quasi-neutrality condition and the perpendicular Ampere's law can be written as

$$
\varphi_{\|} e\left(\frac{k_{\|}^{2}}{\omega^{2}}-\frac{1}{u^{2}}\right)+\frac{b_{\|}}{B} \Delta=0, \quad \frac{B b_{\|}}{4 \pi}+e \varphi_{\|} n_{\mathrm{h}}=0
$$

with

$$
\frac{1}{u^{2}}=\left(\frac{n_{\mathrm{c}}}{m_{\mathrm{e}}}+\frac{n_{\mathrm{i}}}{m_{\mathrm{i}}}\right)^{-1} \frac{n_{\mathrm{eh}}}{m_{\mathrm{e}} V_{\| \mathrm{h}}^{2}}, \quad \Delta=\left(\frac{n_{\mathrm{c}}}{m_{\mathrm{e}}}+\frac{n_{\mathrm{i}}}{m_{\mathrm{i}}}\right)^{-1}\left(n_{\mathrm{i}}-n_{\mathrm{h}}\right),
$$

where $V_{\| \mathrm{h}}$ is the longitudinal velocity of hot electrons. Since Landau damping effects are not relevant here, all imaginary terms are assumed to be small, and can be omitted in Eq. (83).

Further, let us consider the particular case when the cold electron number is much smaller than the hot electron number

$$
n_{\mathrm{c}} \ll n_{\mathrm{h}} \frac{m_{\mathrm{e}}}{m_{\mathrm{i}}} .
$$

Then, from Eq. (83) we have after some algebra:

$$
\omega^{2}=k_{\|}^{2} \frac{V_{\mathrm{s}}^{2} V_{\mathrm{A}}^{2}}{V_{\mathrm{s}}^{2}+V_{\mathrm{A}}^{2}},
$$

Eq. (85) coincides with the MHD slow magnetoacoustic dispersion relation.

It is evident from these calculations that contrary to the basic assumptions of MHD theory the parallel electric field of the wave plays a key role in formation of the slow mode in a collisionless plasma, providing the key momentum transfer mechanism. In fact, the slow mode in kinetics is an electrostatic mode associated with $e_{\|}$and modified by the compression of the magnetic field. Magnetic field non-uniformity complicates the situation due to bounce effects (Le Contel et al. 2000). In this case, the electrostatic mode dispersion properties, known as the Kadomtsev-Pogutse mode, have nothing in common with given by Eq. (85) (Kadomtsev and Pogutse 1967).

The condition given by Eq. (84) is not likely to be satisfied in space plasmas, so let us consider the opposite case:

$$
n_{\mathrm{c}} \gg n_{\mathrm{h}} \frac{m_{\mathrm{e}}}{m_{\mathrm{i}}}, \quad n_{\mathrm{c}} \ll n_{\mathrm{h}} .
$$

In this case, the expressions for $\Delta$ and $u^{2}$ become $\Delta=n_{\mathrm{i}} m_{\mathrm{e}} / n_{\mathrm{c}}$ and $u^{2}=\left(n_{\mathrm{c}} / n_{\mathrm{h}}\right) V_{\| \mathrm{h}}^{2}$ and the set of equations (83) can be combined into a single equation

$$
\left(\frac{k_{\|}^{2}}{\omega^{2}}-\frac{1}{u^{2}}\right)-\frac{4 \pi m_{\mathrm{e}}}{B^{2}} \frac{n_{\mathrm{i}} n_{\mathrm{h}}}{n_{\mathrm{c}}}=0 .
$$

The solution to this equation is a mode with a dispersion relation similar to Eq. (85), but with a different phase speed, $u$ :

$$
\omega^{2}=k_{\|}^{2} u^{2},
$$


where $u^{2} / V_{\mathrm{s}}^{2}=n_{\mathrm{c}} m_{\mathrm{i}} / n_{\mathrm{h}} m_{\mathrm{e}} \gg 1$. Consequently, its frequency is very high and can overstep the limits of the ULF range. When it is said that the cold electron fraction shorts out the parallel electric field it is usually meant that the frequency of the electrostatic mode with the finite $E_{\|}$is too high for the ULF range in the magnetospheric context.

\subsubsection{Drift Compressive Modes}

A nontrivial solution to Eq. (83) appears when the plasma inhomogeneity is taken into account. This oscillation branch is called the drift compressive mode (Ng et al. 1984; Crabtree and Chen 2004; Porazik and Lin 2011). Its characteristic frequency is of the same order as the plasma diamagnetic drift frequency,

$$
\omega_{*}=\frac{k_{y}}{\omega_{\mathrm{c}}} T\left(\frac{n^{\prime}}{n}-\frac{3}{2} \frac{T^{\prime}}{T}\right),
$$

where $n$ is the plasma concentration, and $T$ is the temperature. Probably, the drift compressive mode is the most common high- $m$ compressive mode, since its existence requires only finite $\beta$ and plasma inhomogeneity. The generation of the drift compressive mode can occur due to wave-particle interaction in the presence of a sharp spatial gradient of particle energy (Klimushkin and Mager 2011) and/or an inverted (bump-on-tail) distribution.

The spatial structure of drift compressive modes in a 2D inhomogeneous plasma with parallel and radial non-uniformities was studied by Mager et al. (2013). It was shown that when the wave frequency $\omega$ is much less than the proton bounce frequency $\omega_{\mathrm{b}}$, the mode is narrowly localised along the field line near the equator, being concentrated in the region with high $\beta$. As long as the wave frequency $\omega$ is approaching the drift compressive mode eigenfrequency $\omega_{*}$, the compressive resonance appears, when the field-aligned magnetic field component of the wave field becomes very large. Furthermore, at the compressive resonance the radial component of the wave vector tends to infinity, $k_{x}(x) \rightarrow \infty$, which means that the wave has a large azimuthal magnetic component. This resonance can be considered as a compressive analogue of the MHD Alfvén resonance. However, the radial structure of the drift compressive modes has not been studied in sufficient detail to date.

The drift compressive modes are coupled with the Alfvén modes because of field line curvature and equilibrium current (Klimushkin and Mager 2011). As was shown by Klimushkin et al. (2012), these coupled modes comprise three oscillation branches. The first of them propagates in the azimuthal direction opposite to the diamagnetic drift velocity of ions. It is simply an Alfvén mode weakly modified by the coupling. The two other branches are the Alfvén and drift compressive modes with the azimuthal phase velocity directed parallel to the ion diamagnetic drift velocity. These modes converge to each other with increase in the wave vector azimuthal component $k_{y}$ and the azimuthal current $J$, and merge at some critical values of $k_{y}$ and $J$. If $k_{y}$ and $J$ exceed these critical values, these two branches form two other modes, one of which is unstable, and the other is damped. The unstable mode can be called the drift ballooning coupling mode. Opposite to the ordinary ballooning instability in MHD, the drift ballooning instability is not aperiodic, since there is a real part of the oscillation frequency of the order of the drift frequency. Then, only the mode with the same direction of the azimuthal phase speed as the ion diamagnetic drift velocity can be unstable, and the instability threshold depends on the value of $k_{y}$.

\subsubsection{Drift Mirror Modes}

Compressive ULF waves were also identified as drift-mirror modes (Woch et al. 1990; Rae et al. 2007; Sibeck et al. 2012). These modes can be considered as the drift compressive 
modes in an anisotropic plasma where the particle transverse thermal speed is higher than the parallel thermal speed. This drift-mirror mode is unstable when the parameter $\tau$ is negative (Hasegawa 1969),

$$
\tau=1-\beta_{\perp}\left(\frac{\beta_{\perp}}{\beta_{\|}}-1\right)<0 .
$$

However, the theory of drift-mirror modes is far from complete. For example, the pioneering works of Cheng and Lin (1987), Cheng and Qian (1994) on the effect of parallel inhomogeneity need to be further developed. Even if near the equator the mirror instability condition is satisfied, $\tau<0$, it becomes close to $\tau \simeq 1$ near the ionosphere because the $\beta$ parameter quickly grows towards the ionosphere due to field line convergence. Thus, somewhere on field lines the $\tau$ value must pass through zero. The mode structure and the stability in this case have not been studied to date. Other open questions include

- A realistic kinetic theory of the spatial structure of compressive modes, such as driftcompressive and drift-mirror modes, taking into account their coupling with Alfvén modes must be developed.

- Probably, these waves emerge in the magnetosphere owing to some impulsive processes in space plasmas. Any theory of such process is still lacking.

\subsection{High-Frequency Effects on Coronal MHD Waves}

In the solar corona, high-frequency processes that come into play when the frequencies of MHD oscillations approach the ion gyrofrequency $\Omega_{\mathrm{i}}$ and the spatial scale approaches the ion gyroradius, still remain unresolved. These effects include the Hall dispersion, electron inertia, appearance of kinetic Alfvén waves, ion gyroresonances, splitting of the dispersive branches of left- and right-hand polarised Alfvén waves, etc. A special interest in this phenomenology is connected with the intrinsic possibility for creation of small scales in MHD waves because of the effects of Alfvén phase mixing, resonant absorption (see Sec. 5.5), and nonlinear steepening, and also in the vicinity of various current sheets.

Theoretical studies show that high-frequency effects can play a crucial role in MHD wave energy conversion in the corona. In particular, dispersive Alfvén waves, occurring essentially in the high-frequency regime, were shown to result in nonlinear vortices that were associated with plasma structures observed in the chromosphere, transition region, and in the lower corona. This effect was suggested to be connected with the energy transfer from the lower layers of the solar atmosphere into the corona and, hence, contribute to coronal heating (Sharma et al. 2014).

Voitenko (1998) developed a theoretical scenario of the excitation of kinetic Alfvén waves (KAWs) by warm proton beams accelerated in a flaring coronal loop and flowing down at a super-Alfvénic speed along the just-reconnected field lines, via a beam-induced electric field. KAWs are essentially two-dimensional in the sense that they have long wavelengths along the magnetic field (i.e. small wave numbers $k_{\|}$) and short wavelengths across it (high $k_{\perp}$ ). The model is based on the assumption that the wave frequency is in the vicinity of the ion gyrofrequency, providing the plasma to be still fully electrically neutral, but taking the electron mass to be small, but finite. In the limit $k_{\perp} r_{\mathrm{i}} \gg 1$, where $r_{\mathrm{i}}$ is the ion gyroradius, and $T_{\mathrm{e}}$ and $T_{\mathrm{i}}$ are the electron and ion temperatures, respectively, the dispersion relation for the oblique kinetic Alfvén waves states

$$
\omega \propto k_{\|} k_{\perp}\left(1+\frac{T_{\mathrm{e}}}{T_{\mathrm{i}}}\right)^{1 / 2} .
$$


The consequent evolution of the excited waves can lead to the development of the kinetic Alfvénic instability, generating an anomalous resistivity and efficiently affecting processes of magnetic energy transferring.

If there is an Alfvén speed gradient across the magnetic field, the Alfvén waves are subject to phase mixing, leading to the enhanced collisional wave dissipation, which operates like the efficient Ohmic dissipation of the parallel wave current. Although for usual coronal conditions the dissipation transverse length $\left(\sim 10^{2} \mathrm{~m}\right)$ is much longer than the ion gyroradius $r_{\mathrm{i}}(\sim 1 \mathrm{~m})$, its finite length can cause crucial nonlinear decaying effects, which do not appear in the ideal MHD, if the wave frequency $\omega>1 \mathrm{~Hz}$ (Voitenko and Goossens 2000). The effect of nonlinear mode coupling of phase-mixed Alfvén waves was analytically modelled in frames of two-fluid description accounting for the finite ion gyroradius. It was shown that the growing spectral anisotropy caused by phase mixing initiates nonlinear cascading in the spectral domain, resulting in the appearance of two daughter KAWs. These waves are able to propagate either parallel to each other or to be counter-streaming. Such non-trivial spectral redistribution of the wave energy in turn changes the rate of the dissipation of the wave flux. In particular, for phase-mixed Alfvén waves in a coronal hole, with the amplitude of about 1-3\%, this effect is switched on at the length scales that are much longer than both the dissipation transverse length and the ion gyroradius for all wave processes with frequencies higher than $1 \mathrm{~Hz}$. The efficiency of the effect is proportional to $\omega^{-2 / 3}$.

Modelling of Alfvén wave phase mixing in the kinetic regime (Tsiklauri et al. 2005) demonstrated the creation of compressive perturbations, which caused effective acceleration of electrons due to wave-particle interaction through the oblique, nearly parallel to the magnetic field, electric field induced by the stretching of the phase-mixed Alfvén waves front.

Another high-frequency MHD process, potentially important for the coronal plasma, is the influence of the Hall effect on the nonlinear compressive (e.g. fast magnetoacoustic) wave propagation and reconnection at an X-type magnetic null point. The role of the Hall term in the evolution of an initial fast magnetoacoustic wave and its interaction with topologically varying magnetic environment was investigated in Threlfall et al. (2012). The initial fast magnetoacoustic wave was found to deform rapidly, resulting into coupling of whistler and ion-cyclotron components. The dispersive nature of whistler waves leads to earlier approaching of them the magnetic null point and subsequent creation of multiple additional transient nulls and, hence, an increased number of energy release sites. In the Hall regime, the estimated relevant timescales of the onset of reconnection and the period of the oscillatory relaxation of the system are reduced significantly, and the reconnection rate is enhanced. The latter is very important in the context of the rapid energy releases observed in solar flares.

\section{Is the Direct Impact of Solar MHD Oscillations on the Magnetosphere Possible?}

The signal of oscillatory processes in the solar corona, especially in solar flares, can reach the Earth in a form of periodic modulation of the physical conditions in the solar wind and high energy particle distributions, and by the direct grilling of the ionosphere and upper atmosphere by soft and hard X-rays, gamma-rays and microwaves. These processes may constitute a periodic driver to processes in the geophysical systems, with a possibility for 
a resonance. Thus a question of the possible enhanced geoeffectiveness of these processes, magnified by the resonances, arises.

Kepko and Spence (2003) found several cases of high correlation between the pressure oscillations detected in the solar wind and magnetic oscillations inside the magnetosphere in the frequency range of $0.5-2 \mathrm{mHz}$. They interpreted this finding as an evidence for the forced magnetospheric breathing under the action of slow (period $<10 \mathrm{~min}$ ) magnetoacoustic fluctuations in the wind. For higher frequencies, $2-5 \mathrm{mHz}$, high and stable throughout the solar cycle correlation between the wave amplitudes in the solar wind and on-ground magnetic fluctuations was found in Potapov (2013) suggesting direct penetration of these oscillations into the magnetosphere that operates as a passive or active filter. A theory for the penetration of fast magnetoacoustic waves from the solar wind into the magnetosphere through a plane transition layer was constructed by Leonovich et al. (2003). In particular it was found that in the magnetotail region about $40 \%$ of the energy flux of the incident waves penetrate to the magnetosphere's interior.

On the other hand, a number of authors (e.g. Thomson et al. 2001) reported the finding of some correlation of the solar wind fluctuations and global oscillations of the solar surface (i.e., $p$-modes and possible $g$-modes) in the lower frequency range, below $3 \mathrm{mHz}$. Thus a question arises whether the 3-min and 5-min oscillations typical for the Sun are related to the ULF waves registered in the solar wind near the Earth orbit? This is not an idle question. The role of the most geoeffective parameter in this context is played by the southward component of the interplanetary magnetic field. ULF waves, if they are in fact transported from the solar surface by the solar wind to the Earth's orbit, might modulate the southward component magnitude of the IMF, thus affecting the geomagnetic disturbance regime.

Global 5-min solar surface oscillations and 3-min oscillations ubiquitous in the chromosphere (and most pronounced in sunspot's umbral chromosphere, with the umbra being the dark, central part of a sunspot) are well-known solar phenomena. There is some observational evidence of the possible leakage of 5-min oscillations in the corona (e.g. Tomczyk et al. 2007; De Moortel 2009). Chromospheric 3-min oscillations were found to modulate impulsive flaring energy releases (Sych et al. 2009). Similarly, various oscillations intrinsic for the corona, with the periods ranging from one minute (e.g. in the recently discovered rapidly-propagating wave trains in coronal plasma fans and funnels, Sect. 7.4) to several tens of minutes (e.g. longitudinal waves in coronal holes, Sect. 7.1) could reach the solar wind and approach the Earth. If this hypothesis is proved, then the Sun does exert some quasi-periodic influence on the near-Earth space in the ULF range.

In particular, a possible connection of MHD waves observed in the corona (see, e.g. Sect. 7) and magnetic field oscillations detected in the interplanetary medium was established (Potapov et al. 2013). This effect appears to be quite important too, since the presence of large-scale ULF waves in the solar wind can increase geoeffectiveness of high speed streams in the interplanetary plasma. In the study of Potapov et al. (2013), observations of coronal oscillations in the coronal green Fe I $6569 \AA$ spectral line in a coronal hole were taken as the basis. The high-speed solar wind stream ejected from the coronal hole reached the Earth's orbit after approximately $60 \mathrm{~h}$. The spectra of the oscillations detected in the coronal hole were compared with those of ULF oscillations of the IMF at Lagrangian point L1 between the Earth and the Sun. The oscillations were recorded with the ACE magnetometer when the leading edge of the high speed stream, bringing the enhanced ULF wave activity, reached the Earth. The spectrum of the solar oscillations had a sharp peak at about 3.4$3.6 \mathrm{mHz}$. The spectrum of the solar wind ULF oscillations was found to have this spectral peak too, but was much richer, being either formed by several different sources or representing the evolution of the original spectrum during the wave propagation. Nevertheless, ULF 
oscillations of the IMF often had peaks that are apparently close to the frequencies detected on the solar surface or coronal oscillations. Analysis of the ULF wave spectra observed in ninety two high-speed streams confirmed the presence of 3-min and 5-min oscillations in the total wave spectrum. Certainly, these results cannot be regarded as proving a direct connection between solar oscillations and ULF waves at the Earth's orbit even though they do support such a possibility. Making more definite conclusions proving or rejecting this hypothesis would require additional research including, e.g. the wave trajectory and evolution calculations in the interplanetary medium if we assume not only transportation of waves by the solar wind, but their propagation as well.

The possibility of geomagnetic precursors of large, geoeffective solar flares-long-period variations of the geomagnetic field - has been studied in Kobrin et al. (1985). These precursors are global enhancements of geomagnetic variations with time scales 20-60 min about 1-3 days before the flare. For example, before an X9 flare on 22 March 1991 the flux of Xray emission demonstrated elevated level $\left(\sim 2.5 \times 10^{-12} \mathrm{~W}\right)$ and varied quasi-periodically with the scale $30-60 \mathrm{~min}$. The correlation coefficient between these variations and oscillations of the geomagnetic field was very high, reaching 0.92 for low-latitude stations, and 0.84 for mid-latitude stations. The intensity of these geomagnetic oscillations was found to increase with the increase in the solar proton fluxes. Indeed, large-scale variations with these periods are occasionally seen in different layers of the solar atmosphere in different observational bands (see, e.g. Abramov-Maximov et al. 2013; Yuan et al. 2011). But, association of these oscillations with flares has not been established. Anyway, the long-period solar atmospheric oscillations are known to modulate emission of the solar coronal plasma in the radio, EUV, and X-ray bands. Solar EUV and soft X-ray emissions produce the additional ionisation of the terrestrial ionosphere, and consequently affect the ionospheric currents and geomagnetic field. Thus, it is natural to expect that time variability of the solar emission can appear in the terrestrial observables. Moreover, in the case of quasi-periodic variations of the solar flux one could expect various resonances. This topic certainly deserves a more detailed study, especially the role of QPPs in the geoeffectiveness of solar flares.

An additional possibility for the direct linkage of MHD oscillations in the solar corona and oscillatory processes in the Earth's magnetosphere is connected with quasi-periodic pulsations (QPPs, see Sect. 11.2) in flaring light curves. As it is discussed in Sect. 11, several mechanisms could be responsible for the appearance of QPPs in microwave, and soft and hard X-ray radiation of a solar flare. Moreover, observations clearly show that QPPs is a common, and perhaps even an intrinsic feature of flaring energy releases. Similar oscillatory and self-oscillatory processes may occur in various plasma configurations of the Earth's magnetosphere, e.g. at the dayside reconnection site, and in the magnetotail, thus there is a possibility for a resonance.

In particular, the ionospheric vulnerability to UV and X-ray bursts from a solar flare is named a solar flare effect (SFE, see, e.g. Mitra 1974). Rapid variations of the ionospheric plasma density causes a complex of phenomena, called sudden ionospheric disturbances (SID). The SID probability increases with the growth of the X-ray flare energy, reaching about $90 \%$. Magnetospheric eigenoscillations could be excited by a sudden change of the conductivity of the magnetospheric resonator boundary, the ionosphere. Solar flares can cause not only ionisation, but also strong impulsive heating of the lower ionosphere. This could result in the acoustic-gravity waves excitation in the upper atmosphere. Efficiency of these processes may be increased in the case of a resonance between the natural frequencies and the periodicity of the excitation, i.e. QPPs in flares.

A quasi-periodic response of the magnetosphere is observed by ground magnetometers in various frequency ranges, for the periods from a few seconds to several minutes, named 
P1-3sfe pulsations. These geomagnetic pulsations are observed when the flare exceeds the M4 class (e.g. Parkhomov et al. 2006, 2008). These periodic responses could be attributed to magnetospheric or ionospheric resonant modes. Spectral characteristics of irregular geomagnetic pulsations associated with hard X-rays flares were examined by Lukovnikova and Parkhomov (1984). Observational data obtained with a chain of ground-based magnetometers, for flares over the 1965-1976 year period were analysed, and peak pulsation values with periods of 40,100, and $300 \mathrm{~s}$ were found. Probably, pulsations were induced by three different mechanisms: current fluctuations in the dynamo region of the ionosphere, magnetospheric resonant oscillations, and acoustic waves. However, these suggestions have not been supported yet by detailed data analysis or theoretical modelling. Thus, the relevant physical mechanisms are poorly understood and require detailed modelling and analysis.

\section{Conclusions and Perspectives}

In this review we have summarised the main observational manifestation and properties of MHD waves in the corona and the magnetosphere, and main results of their theoretical modelling. Despite a similarity between the approaches in solar and magnetospheric physics for the description of wave processes - they are based on the same MHD theory-there are some fundamental differences between coronal and magnetospheric waves as well. In the solar corona, the plasma is the field-aligned filamented and structured, e.g. into closed elongated flux tubes, the loops. Thus, the basic building block of the theory of coronal MHD wave processes is the plasma cylinder oscillations. In the magnetosphere, the plasma is rather homogeneous at least in one direction (azimuthal), so the elemental wave process is oscillations of magnetic shells.

Another difference is that in the magnetosphere, in the entire ULF frequency range, the plasma can be treated as collisionless. At the same time, in the solar atmosphere, some oscillatory modes are in collisional regime (i.e. wavelengths of observed waves, including slow magnetoacoustic waves, exceed the proton mean free path), whereas other modes correspond to a collisionless regime. As a result, in the corona there is a vast observational evidence of slow magnetoacoustic waves (see Sects. 6.3 and 7.1) that in the low- $\beta$ plasma could degenerate to pure acoustic waves, absent in the collisionless magnetospheric plasma. In the magnetosphere, the lack of high-quality slow mode waves is connected with strong Landau damping, so this mode can be involved in wave processes only as a coupled mode (e.g., poloidal or ballooning modes).

Many long-period MHD wave phenomena in the magnetosphere are global, and involve either the whole magnetosphere (e.g., cavity modes) or its significant part in the wave motion. Thus, their properties are determined by the global geometry of the magnetospheric magnetic field and plasma distribution. In contrast, coronal MHD waves are always of the local nature. Even the events called global coronal waves (Sect. 2) develop from a welldefined epicentre. Moreover, there is still a debate whether these events are connected with MHD waves at all.

Nonetheless, there are plasma objects and configurations in the magnetosphere where the notions developed in the solar physics could be applied. On the dusk side, just outside the nominal plasmapause location, clouds of detached plasmaspheric plasma are regularly observed. Such clouds, in the first approximation, can be considered as flux tubes filled with the dense ionospheric plasma. The possible oscillations of the detached plasma tubes can result in the occurrence of specific localised ULF wave activity in this region. For their identification the models elaborated in the solar physics can be used. 
This review identifies several other research topics that would definitely benefit from the knowledge transfer and cross-fertilisation of magnetospheric and coronal MHD wave studies.

The effect of turbulent motions on the MHD wave excitation and propagation needs to be understood. In particular, the excitation of almost monochromatic oscillations such as the decay-less kink oscillations of coronal loops and magnetospheric field-line resonances seem to be related to a similar excitation mechanism. It is the excitation of natural modes of a resonator (actually, predominantly the global mode in both the coronal and magnetospheric cases) by a continuously-operating broadband pumping.

Linear coupling of fast magnetoacoustic and Alfvén waves at the singular resonant surface where the phase speed of the fast waves equals the local Alfvén speed, plays an important role in both magnetospheric and coronal applications. In the magnetospheric applications this effect is directly observed, while in the corona it is still a theoretical model (see Sect. 5.5). It seems to be highly beneficial for both the research communities to perform a detailed comparative study of this effect.

The magnetospheric poloidal Alfvén waves with the large azimuthal wavenumber, slowly propagating sunward in the azimuthal direction are strongly compressive. Moreover, these waves are not fast magnetoacoustic, as the perturbation of the plasma density is seen in antiphase with the perturbations of the absolute value of the magnetic field. This phenomenon seems to be similar to the magnetoacoustic wave progressing along the neutral line in tworibbon flares, discussed in Sect. 6.3.

A cusp, either appearing above flaring arcades and in helmet streamers in the corona, or the magnetospheric polar cusp, acts as an accumulation region for fast magnetoacoustic wave energy in both the physical systems. This phenomenon deserves deep investigation both theoretically and observationally, as, in particular, it may be responsible for the formation of quasi-periodic patterns in magnetic energy releases.

The geometry of the Earth's magnetotail is similar to the geometry of coronal helmet streamers. Both these plasma systems are observed to guide MHD waves. Both the systems constitute two waveguides, one is the current sheet (the "warm" waveguide), and the other is the density enhancement surrounding the current sheet (the "cold" waveguide). Discrimination between the modes guided by the different waveguides is an interesting problem, with potential seismological implications. A similar magnetic configuration, a vertical current sheet above the reconnection site, is predicted by the standard model of solar flares. The search for MHD waves in this system is an interesting task that is important for comparative studies.

The effect of virtual compressive resonances has been studied in both magnetospheric and coronal contexts, however independently. The knowledge transfer between our research fields would be highly beneficial, including analytical models that have exact analytical solutions and approximate methods for the analysis of more general cases.

The integral emission curves recorded in both magnetospheric sub-storms and solar flares show quasi-periodic pulsations. The physical mechanisms responsible for QPPs need to be revealed. They may be either associated with MHD oscillations or quasi-periodic regimes of magnetic reconnection (the magnetic dripping, i.e. "pulsed" or "repetitive" reconnection). In the former case, one has to explain how low-amplitude oscillations produce high-amplitude variations of the emission intensity. In the latter case, one needs to determine the conditions for the appearance of this regime, and the relationship of the period with the parameters of the reconnecting current sheet and its environment.

For magnetospheric ULF waves, an important role is played by the partially-ionised layer of the ionospheric plasma at the footpoints of the oscillating magnetic field lines. In the solar 
corona a similar effect can be caused by the partial ionisation of the chromospheric plasma at the footpoints of, e.g., coronal loops. Some consolidated understanding of the role of the partial ionisation needs to be achieved.

In solar coronal loops, the non-equidistant frequency spectrum of standing kink oscillations is attributed to the non-uniformity of the Alfvén speed with height. Multi-band spectral features of magnetospheric ULF waves, associated with magnetospheric or ionospheric Alfvénic resonators, are used as a reliable tool for the diagnostics of the plasma distribution along geomagnetic field lines. It would be interesting to combine the analytical techniques and methods for the development and application of plasma diagnostics techniques.

ULF variations of the terrestrial electromagnetic field are effective tools for a continuous monitoring of dynamical phenomena in the magnetosphere, ionosphere, and atmosphere. The advanced understanding of ULF wave physics enabled space physicists to develop reliable methods of "hydromagnetic seismology" of the outer space to monitor the plasma density variations in the magnetosphere and ionosphere. However, in contrast with the similar usage of seismic waves for the solid Earth probing, in magnetospheric physics particular sources of ULF disturbances and their spectral properties are practically unknown. Nevertheless, the resonant transformation process, which is inevitably related to the propagation of MHD disturbances from extra-magnetospheric regions to the ground, ensures the existence of a specific resonant frequency for any geomagnetic location. The ground-based methods of hydromagnetic diagnostics of magnetospheric plasma are based on the extraction of this resonant frequency from ULF spectra. Similar techniques, jointly called MHD seismology of the solar corona, are intensively developed and used in the diagnostics of the coronal plasma. In particular, it provides the unique information about the coronal magnetic field, transport coefficients, the adiabatic index, sub-resolution structuring, and the characteristic scale of the stratification.

Despite intensive studies and numerous papers on ULF waves in the magnetosphere, many vital problems still cannot be considered as resolved. Most theoretical models are oversimplified and take into account only some aspects of actual physical phenomena. For example, theoretical models either describe realistic MHD modes, neglecting kinetic effects, or consider wave-particle interaction assuming a simple plane wave approximation. In reality, the integral growth rate of ULF waves is to be determined not only by wave-particle interaction, but the spatial structure of unstable modes.

Traditionally, wave processes in those two space objects- the Earth and Sun, have been studied so far almost independently. We attempted to outline main fundamental MHD wave processes which may occur in these two plasma systems. In addition, well-elaborated physical models designed by the magnetospheric wave communities, which, in our opinion, have a wider application area including solar, space and astrophysical plasma, but have so far obtained very limited attention, comprise the following physical phenomena:

- spectral magnetohydrodynamics, describing discrete and continuous spectra and eigenfunctions of MHD waves in an inhomogeneous plasma immersed into curved magnetic field;

- interaction of MHD waves with a multi-layer interface between the collisionless plasma and non-conductive atmosphere; the occurrence of transmission windows on the frequency domain owing to the ionospheric Alfvénic resonator and MHD waveguide;

- spontaneous excitation of small-scale Alfvénic modes by kinetic instabilities of energetic plasma component;

- Alfvénic energisation of warm electrons owing to kinetic or mirror resistance effects;

- the interaction due to a finite-frequency effect of propagating magnetoacoustic and Alfvén waves in a longitudinally inhomogeneous plasma in regions with open field lines. 
In general, the potentials of MHD waves for the monitoring of plasma processes in nearEarth space and solar corona have not been fully exploited yet, and MHD seismology of the magnetosphere and corona just originated. MHD seismological techniques have a great potential in real-time monitoring the physical conditions in the corona and the near-Earth environment, with very interesting implications for space weather, which needs to be revealed and exploited.

Acknowledgements This review is based upon the activities of the international science team "MHD oscillations in the solar corona and Earth's magnetosphere: towards consolidated understanding" supported by the International Space Science Institute, Bern, Switzerland. The authors would like to thank Dr N. Nishitani and Dr A. Yoshikawa for the valuable discussions. The authors acknowledge the support by the STFC Warwick Astrophysics Consolidated Grant ST/L000733/1 (VMN, GN); the European Research Council under the SeismoSun Research Project No. 321141 (VMN), BK21 plus program through the National Research Foundation funded by the Ministry of Education of Korea (DHL, VMN); the Leverhulme Trust (GV); the János Bolyai Research Scholarship of the Hungarian Academy of Sciences (BH); grant P209/12/0103 and 16-13277S (GA CR) (MK, PJ); an Odysseus grant of the FWO Vlaanderen, the IAP P7/08 CHARM (Belspo) and the GOA-2015-014 (KU Leuven) (TVD); Program No 9 of the Presidium of the Russian Academy of Sciences (DYK).

\section{Appendix}

Abbreviations and acronyms commonly used in solar coronal and Earth's magnetospheric physics:
AAR Auroral acceleration region
AIA Atmospheric Imaging Assembly on SDO
BBF bursty bulk flow events
$\mathrm{CBF} \quad$ coronal bright fronts
CIR Corotating interaction region
CME Coronal mass ejection
CSHKP Carmichael, Sturrock, Hirayama, Kopp-Pneuman (or standard) model of a solar flare
EIS Extreme Ultraviolet Imaging Spectrometer on Hinode
EIT Extreme Ultraviolet Imaging Telescope on SoHO
EMIC Electromagnetic ion-cyclotron
EUV Extreme Ultraviolet
FLR field line resonance — an eigenmode of a closed geomagnetic field line
FMS Fast magnetosonic
FTE Flux transfer events
IAR Ionospheric Alfvénic resonator
IMF Interplanetary magnetic field
IRI International Reference Ionosphere model
KAWs Kinetic Alfvén waves
KHI Kelvin-Helmholtz instability
LLBL Low latitude boundary layer
LoS Line-of-sight
MHD Magnetohydrodynamic
MP Magnetopause
MSIS Mass Spectrometer-Incoherent Scatter Model of the upper atmosphere
NFTE Nightside flux transfer events
NoRH Nobeyama Radioheliograph 


$\begin{array}{ll}\text { NRH } & \text { Nancey RadioHeliograph } \\ \text { QPPs } & \text { Quasi-periodic pulsations } \\ \text { RHESSI } & \text { Reuven Ramaty High-Energy Solar Spectroscopic Imager } \\ \text { SDO } & \text { NASA Solar Dynamics Observatory } \\ \text { SFE } & \text { Solar flare effect } \\ \text { SI } & \text { Sudden impulse } \\ \text { SID } & \text { Sudden ionospheric disturbance } \\ \text { SoHO } & \text { ESA/NASA Solar and Heliospheric Observatory } \\ \text { SSC } & \text { Storm sudden commencement } \\ \text { SSRT } & \text { Siberian Solar Radio Telescope } \\ \text { STEREO } & \text { Solar TErrestrial RElations Observatory } \\ \text { SUMER } & \text { Solar Ultraviolet Measurements of Emitted Radiation instrument on SOHO } \\ \text { SXT } & \text { Solar X-ray Telescope on Yohkoh } \\ \text { TRACE } & \text { Transition Region and Coronal Explorer } \\ \text { ULF } & \text { Ultra low frequency } \\ \text { XRT } & \text { X-ray Telescope on Hinode. }\end{array}$

\section{References}

V.E. Abramov-Maximov, V.I. Efremov, L.D. Parfinenko, A.A. Solov'ev, K. Shibasaki, Long-term oscillations of sunspots from simultaneous observations with the Nobeyama radioheliograph and solar dynamics observatory. Publ. Astron. Soc. Jpn. 65, 12 (2013). doi:10.1093/pasj/65.sp1.S12

A.N. Afanasyev, V.M. Nakariakov, Nonlinear slow magnetoacoustic waves in coronal plasma structures. Astron. Astrophys. 573, 32 (2015). doi:10.1051/0004-6361/201424516

A.N. Afanasyev, A.M. Uralov, Coronal shock waves, EUV waves, and their relation to CMEs. II. Modeling MHD shock wave propagation along the solar surface, using nonlinear geometrical acoustics. Sol. Phys. 273, 479-491 (2011). doi:10.1007/s11207-011-9730-9

H. Alfvén, Existence of electromagnetic-hydrodynamic waves. Nature 150, $405-406$ (1942). doi:10.1038/ $150405 \mathrm{~d} 0$

L.S. Alperovich, E.N. Fedorov (eds.), in Hydromagnetic Waves in the Magnetosphere and the Ionosphere. Astrophysics and Space Science Library, vol. 353 (2007)

J. Andries, T. van Doorsselaere, B. Roberts, G. Verth, E. Verwichte, R. Erdélyi, Coronal seismology by means of kink oscillation overtones. Space Sci. Rev. 149, 3-29 (2009). doi:10.1007/s11214-009-9561-2

S. Anfinogentov, G. Nisticò, V.M. Nakariakov, Decay-less kink oscillations in coronal loops. Astron. Astrophys. 560, 107 (2013). doi:10.1051/0004-6361/201322094

P. Antolin, T. Van Doorsselaere, Line-of-sight geometrical and instrumental resolution effects on intensity perturbations by sausage modes. Astron. Astrophys. 555, 74 (2013). doi:10.1051/0004-6361/ 201220784

P. Antolin, T. Yokoyama, T. Van Doorsselaere, Fine strand-like structure in the solar corona from magnetohydrodynamic transverse oscillations. Astrophys. J. Lett. 787, 22 (2014). doi:10.1088/2041-8205/ $787 / 2 / \mathrm{L} 22$

I. Arregui, Wave heating of the solar atmosphere. Philos. Trans. R. Soc. Lond. Ser. A 373, 40261 (2015). doi:10.1098/rsta.2014.0261

I. Arregui, J. Andries, T. Van Doorsselaere, M. Goossens, S. Poedts, MHD seismology of coronal loops using the period and damping of quasi-mode kink oscillations. Astron. Astrophys. 463, 333-338 (2007). doi:10.1051/0004-6361:20065863

A. Artemyev, I. Zimovets, Stability of current sheets in the solar corona. Sol. Phys. 277, 283-298 (2012). doi:10.1007/s11207-011-9908-1

A. Asai, T.T. Ishii, H. Isobe, R. Kitai, K. Ichimoto, S. UeNo, S. Nagata, S. Morita, K. Nishida, D. Shiota, A. Oi, M. Akioka, K. Shibata, First simultaneous observation of an $\mathrm{H} \alpha$ Moreton wave, EUV wave, and filament/prominence oscillations. Astrophys. J. Lett. 745, 18 (2012). doi:10.1088/2041-8205/745/2/L18

M.J. Aschwanden, Theory of radio pulsations in coronal loops. Sol. Phys. 111, 113-136 (1987). doi:10.1007/ BF00145445

M.J. Aschwanden, L. Fletcher, C.J. Schrijver, D. Alexander, Coronal loop oscillations observed with the transition region and coronal explorer. Astrophys. J. 520, 880-894 (1999). doi:10.1086/307502 
G.D.R. Attrill, L.K. Harra, L. van Driel-Gesztelyi, P. Démoulin, Coronal "Wave": magnetic footprint of a coronal mass ejection? Astrophys. J. Lett. 656, 101-104 (2007). doi:10.1086/512854

G. Aulanier, M. Janvier, B. Schmieder, The standard flare model in three dimensions. I. Strong-to-weak shear transition in post-flare loops. Astron. Astrophys. 543, 110 (2012). doi:10.1051/0004-6361/201219311

L.J. Baddeley, T.K. Yeoman, D.M. Wright, J.A. Davies, K.J. Trattner, J.L. Roeder, Morning sector driftbounce resonance driven ULF waves observed in artificially-induced HF radar backscatter. Ann. Geophys. 20, 1487-1498 (2002). doi:10.5194/angeo-20-1487-2002

D. Banerjee, E. O'Shea, J.G. Doyle, M. Goossens, Long period oscillations in the inter-plume regions of the Sun. Astron. Astrophys. 377, 691-700 (2001). doi:10.1051/0004-6361:20011153

D. Banerjee, G.R. Gupta, L. Teriaca, Propagating MHD waves in coronal holes. Space Sci. Rev. 158, 267-288 (2011). doi:10.1007/s11214-010-9698-z

M. Bárta, J. Büchner, M. Karlický, J. Skála, Spontaneous current-layer fragmentation and cascading reconnection in solar flares. I. Model and analysis. Astrophys. J. 737, 24 (2011). doi:10.1088/0004-637X/ $737 / 1 / 24$

T.M. Bauer, W. Baumjohann, R.A. Treumann, N. Sckopke, H. Lühr, Low-frequency waves in the near-Earth plasma sheet. J. Geophys. Res. 100, 9605-9618 (1995). doi:10.1029/95JA00136

A.J.C. Beliën, P.C.H. Martens, R. Keppens, Coronal heating by resonant absorption: the effects of chromospheric coupling. Astrophys. J. 526, 478-493 (1999). doi:10.1086/307980

A.O. Benz, Flare observations. Living Rev. Sol. Phys. 5, 1 (2008). doi:10.12942/lrsp-2008-1

D. Berghmans, F. Clette, Active region EUV transient brightenings-first results by EIT of SOHO JOP80. Sol. Phys. 186, 207-229 (1999). doi:10.1023/A:1005189508371

D. Berghmans, P. de Bruyne, Coronal loop oscillations driven by footpoint motions: analytical results for a model problem. Astrophys. J. 453, 495 (1995). doi:10.1086/176410

D.A. Biesecker, D.C. Myers, B.J. Thompson, D.M. Hammer, A. Vourlidas, Solar phenomena associated with "EIT Waves". Astrophys. J. 569, 1009-1015 (2002). doi:10.1086/339402

D.L. Book, NRL Plasma Formulary (Naval Research Lab., Washington, 1983)

J.E. Borovsky, Auroral arc thicknesses as predicted by various theories. J. Geophys. Res. 98, 6101-6138 (1993). doi:10.1029/92JA02242

G.J.J. Botha, T.D. Arber, V.M. Nakariakov, Y.D. Zhugzhda, Chromospheric resonances above sunspot umbrae. Astrophys. J. 728, 84 (2011). doi:10.1088/0004-637X/728/2/84

C.S. Brady, E. Verwichte, T.D. Arber, Leakage of waves from coronal loops by wave tunneling. Astron. Astrophys. 449, 389-399 (2006). doi:10.1051/0004-6361:20054097

R. Bruno, V. Carbone, The solar wind as a turbulence laboratory. Living Rev. Sol. Phys. 10, 2 (2013). doi:10. 12942/lrsp-2013-2

P.S. Cally, Leaky and non-leaky oscillations in magnetic flux tubes. Sol. Phys. 103, $277-298$ (1986). doi:10.1007/BF00147830

K. Chandrashekhar, R.J. Morton, D. Banerjee, G.R. Gupta, The dynamical behaviour of a jet in an ondisk coronal hole observed with AIA/SDO. Astron. Astrophys. 562, 98 (2014). doi:10.1051/0004-6361/ 201322408

C.C. Chaston, J.W. Bonnell, L.M. Peticolas, C.W. Carlson, J.P. McFadden, R.E. Ergun, Driven Alfvén waves and electron acceleration: a FAST case study. Geophys. Res. Lett. 29, 1535 (2002a). doi:10.1029/ 2001GL013842

C.C. Chaston, J.W. Bonnell, C.W. Carlson, M. Berthomier, L.M. Peticolas, I. Roth, J.P. McFadden, R.E. Ergun, R.J. Strangeway, Electron acceleration in the ionospheric Alfvén resonator. J. Geophys. Res. Space Phys. 107, 1413 (2002b). doi:10.1029/2002JA009272

P.F. Chen, The relation between EIT waves and solar flares. Astrophys. J. Lett. 641, 153-156 (2006). doi: $10.1086 / 503868$

P.F. Chen, Coronal mass ejections: models and their observational basis. Living Rev. Sol. Phys. 8, 1 (2011). doi:10.12942/lrsp-2011-1

L. Chen, A. Hasegawa, A theory of long-period magnetic pulsations: 1. Steady state excitation of field line resonance. J. Geophys. Res. 79, 1024-1032 (1974). doi:10.1029/JA079i007p01024

P.F. Chen, E.R. Priest, Transition-region explosive events: reconnection modulated by p-mode waves. Sol. Phys. 238, 313-327 (2006). doi:10.1007/s11207-006-0215-1

P.F. Chen, Y. Wu, First evidence of coexisting EIT wave and coronal Moreton wave from SDO/AIA observations. Astrophys. J. Lett. 732, 20 (2011). doi:10.1088/2041-8205/732/2/L20

P.F. Chen, S.T. Wu, K. Shibata, C. Fang, Evidence of EIT and Moreton waves in numerical simulations. Astrophys. J. Lett. 572, 99-102 (2002). doi:10.1086/341486

P.F. Chen, C. Fang, K. Shibata, A full view of EIT waves. Astrophys. J. 622, 1202-1210 (2005). doi:10.1086/428084

Y. Chen, H.Q. Song, B. Li, L.D. Xia, Z. Wu, H. Fu, X. Li, Streamer waves driven by coronal mass ejections. Astrophys. J. 714, 644-651 (2010). doi:10.1088/0004-637X/714/1/644 
C.Z. Cheng, C.S. Lin, Eigenmode analysis of compressional waves in the magnetosphere. Geophys. Res. Lett. 14, 884-887 (1987). doi:10.1029/GL014i008p00884

C.Z. Cheng, Q. Qian, Theory of ballooning-mirror instabilities for anisotropic pressure plasmas in the magnetosphere. J. Geophys. Res. 99, 11193-11210 (1994). doi:10.1029/94JA00657

J.W. Cirtain, L. Golub, L. Lundquist, A. van Ballegooijen, A. Savcheva, M. Shimojo, E. DeLuca, S. Tsuneta, T. Sakao, K. Reeves, M. Weber, R. Kano, N. Narukage, K. Shibasaki, Evidence for Alfvén waves in solar X-ray jets. Science 318, 1580 (2007). doi:10.1126/science.1147050

S.G. Claudepierre, S.R. Elkington, M. Wiltberger, Solar wind driving of magnetospheric ULF waves: pulsations driven by velocity shear at the magnetopause. J. Geophys. Res. Space Phys. 113, 5218 (2008). doi:10.1029/2007JA012890

F.C. Cooper, V.M. Nakariakov, D. Tsiklauri, Line-of-sight effects on observability of kink and sausage modes in coronal structures with imaging telescopes. Astron. Astrophys. 397, 765-770 (2003a). doi:10.1051/ 0004-6361:20021556

F.C. Cooper, V.M. Nakariakov, D.R. Williams, Short period fast waves in solar coronal loops. Astron. Astrophys. 409, 325-330 (2003b). doi:10.1051/0004-6361:20031071

S.W.H. Cowley, M. Ashour-Abdalla, Adiabatic plasma convection in a dipole field: proton forbiddenzone effects for a simple electric field model. Planet. Space Sci. 24, 821-833 (1976). doi:10.1016/ 0032-0633(76)90072-6

C. Crabtree, L. Chen, Finite gyroradius theory of drift compressional modes. Geophys. Res. Lett. 31, 17804 (2004). doi:10.1029/2004GL020660

R. Cramm, K.-H. Glassmeier, M. Stellmacher, C. Othmer, Evidence for resonant mode coupling in Saturn's magnetosphere. J. Geophys. Res. 103, 11951-11960 (1998). doi:10.1029/98JA00629

S.R. Cranmer, Coronal holes and the high-speed solar wind. Space Sci. Rev. 101, 229-294 (2002)

S.R. Cranmer, A.A. van Ballegooijen, On the generation, propagation, and reflection of Alfvén waves from the solar photosphere to the distant heliosphere. Astrophys. J. Suppl. 156, 265-293 (2005). doi:10.1086/426507

J.M. Davila, Heating of the solar corona by the resonant absorption of Alfvén waves. Astrophys. J. 317, 514-521 (1987). doi:10.1086/165295

I. De Moortel, Longitudinal waves in coronal loops. Space Sci. Rev. 149, 65-81 (2009). doi:10.1007/ s11214-009-9526-5

I. De Moortel, A.W. Hood, The damping of slow MHD waves in solar coronal magnetic fields. Astron. Astrophys. 408, 755-765 (2003). doi:10.1051/0004-6361:20030984

I. De Moortel, A.W. Hood, The damping of slow MHD waves in solar coronal magnetic fields. II. The effect of gravitational stratification and field line divergence. Astron. Astrophys. 415, 705-715 (2004). doi:10.1051/0004-6361:20034233

I. De Moortel, V.M. Nakariakov, Magnetohydrodynamic waves and coronal seismology: an overview of recent results. Philos. Trans. R. Soc. Lond. Ser. A 370, 3193-3216 (2012). doi:10.1098/rsta.2011.0640

I. De Moortel, D.J. Pascoe, The effects of line-of-sight integration on multistrand coronal loop oscillations. Astrophys. J. 746, 31 (2012). doi:10.1088/0004-637X/746/1/31

I. De Moortel, J. Ireland, R.W. Walsh, A.W. Hood, Longitudinal intensity oscillations in coronal loops observed with TRACE I. Overview of measured parameters. Sol. Phys. 209, 61-88 (2002). doi:10.1023/A:1020956421063

B. de Pontieu, G. Haerendel, Weakly damped Alfvén waves as drivers for spicules. Astron. Astrophys. 338, 729-736 (1998)

B. De Pontieu, P.C.H. Martens, H.S. Hudson, Chromospheric damping of Alfvén waves. Astrophys. J. 558, 859-871 (2001). doi:10.1086/322408

B. De Pontieu, R. Erdélyi, I. De Moortel, How to channel photospheric oscillations into the corona. Astrophys. J. Lett. 624, 61-64 (2005). doi:10.1086/430345

C.E. DeForest, J.B. Gurman, Observation of quasi-periodic compressive waves in solar polar plumes. Astrophys. J. Lett. 501, 217-220 (1998). doi:10.1086/311460

C. Delannée, G. Aulanier, CME associated with transequatorial loops and a bald patch flare. Sol. Phys. 190, 107-129 (1999). doi:10.1023/A:1005249416605

A.G. Demekhov, V.Y. Trakhtengerts, T. Bösinger, Pc 1 waves and ionospheric Alfvén resonator: generation or filtration? Geophys. Res. Lett. 27, 3805-3808 (2000). doi:10.1029/2000GL000126

R.E. Denton, Compressibility of the poloidal mode. J. Geophys. Res. 103, 4755-4760 (1998). doi:10. 1029/97JA02652

J. Du, T.L. Zhang, R. Nakamura, C. Wang, W. Baumjohann, A.M. Du, M. Volwerk, K.-H. Glassmeier, J.P. McFadden, Mode conversion between Alfvén and slow waves observed in the magnetotail by THEMIS. Geophys. Res. Lett. 38, 7101 (2011). doi:10.1029/2011GL046989

J.W. Dungey, D.J. Southwood, Ultra low frequency waves in the magnetosphere. Space Sci. Rev. 10, 672-688 (1970). doi:10.1007/BF00171551 
M.M. Echim, J.F. Lemaire, Laboratory and numerical simulations of the impulsive penetration mechanism. Space Sci. Rev. 92, 565-601 (2000)

P.M. Edwin, B. Roberts, Wave propagation in a magnetically structured atmosphere. III. The slab in a magnetic environment. Sol. Phys. 76, 239-259 (1982). doi:10.1007/BF00170986

P.M. Edwin, B. Roberts, Wave propagation in a magnetic cylinder. Sol. Phys. 88, 179-191 (1983). doi:10.1007/BF00196186

R. Erdélyi, Y. Taroyan, Hinode EUV spectroscopic observations of coronal oscillations. Astron. Astrophys. 489, 49-52 (2008). doi:10.1051/0004-6361:200810263

R.E. Ergun, Y.-J. Su, L. Andersson, F. Bagenal, P.A. Delamere, R.L. Lysak, R.J. Strangway, S-bursts and the Jupiter ionospheric Alfvén resonator. J. Geophys. Res. 111, 06212 (2006). doi:10.1029/2005JA011253

N.V. Erkaev, V.S. Semenov, H.K. Biernat, Magnetic double-gradient instability and flapping waves in a current sheet. Phys. Rev. Lett. 99(23), 235003 (2007). doi:10.1103/PhysRevLett.99.235003

N.V. Erkaev, V.S. Semenov, I.V. Kubyshkin, M.V. Kubyshkina, H.K. Biernat, MHD model of the flapping motions in the magnetotail current sheet. J. Geophys. Res. 114, 3206 (2009). doi:10.1029/2008JA013728

N.V. Erkaev, V.S. Semenov, H.K. Biernat, Hall magnetohydrodynamic effects for current sheet flapping oscillations related to the magnetic double gradient mechanism. Phys. Plasmas 17(6), 060703 (2010). doi:10.1063/1.3439687

E.N. Fedorov, V.A. Pilipenko, V.V. Vovchenko, Interaction between the Alfvén wave and turbulent sheet. Geomagn. Aeron. 47, 570-579 (2007). doi:10.1134/S0016793207050052

F.R. Fenrich, J.C. Samson, Growth and decay of field line resonances. J. Geophys. Res. 102, 20031-20040 (1997). doi:10.1029/97JA01376

C.A. Ferraro, C. Plumpton, Hydromagnetic waves in a horizontally stratified atmosphere. V. Astrophys. J. 127, 459 (1958). doi:10.1086/146474

L. Fletcher, H.S. Hudson, Impulsive phase flare energy transport by large-scale Alfvén waves and the electron acceleration problem. Astrophys. J. 675, 1645-1655 (2008). doi:10.1086/527044

C. Foullon, E. Verwichte, V.M. Nakariakov, K. Nykyri, C.J. Farrugia, Magnetic Kelvin-Helmholtz instability at the Sun. Astrophys. J. Lett. 729, 8 (2011). doi:10.1088/2041-8205/729/1/L8

S. Fujita, T. Tanaka, Magnetospheric plasma processes during a sudden commencement revealed from a global MHD simulation, in Magnetospheric ULF Waves: Synthesis and New Directions, ed. by K. Takahashi, P.J. Chi, R.E. Denton, R.L. Lysak. Washington DC American Geophysical Union Geophysical Monograph Series, vol. 169 (2006), p. 31

S. Fujita, M. Itonaga, H. Nakata, Relationship between the Pi2 pulsations and the localized impulsive current associated with the current disruption in the magnetosphere. Earth Planets Space 52, 267-281 (2000)

H. Fukunishi, L.J. Lanzerotti, Hydromagnetic waves in the dayside cusp region and ground signatures of flux transfer events, in Plasma Waves and Instabilities at Comets and in Magnetospheres. Washington DC American Geophysical Union Geophysical Monograph Series, vol. 53 (1989), pp. 179-195

P.T. Gallagher, D.M. Long, Large-scale bright fronts in the solar corona: a review of "EIT waves". Space Sci. Rev. 158, 365-396 (2011). doi:10.1007/s11214-010-9710-7

K.-H. Glassmeier, C. Othmer, R. Cramm, et al., Magnetospheric field line resonances: a comparative planetology approach. Surv. Geophys. 20, 61-109 (1999). doi:10.1023/A:1006659717963

C.K. Goertz, R.W. Boswell, Magnetosphere-ionosphere coupling. J. Geophys. Res. 84, 7239-7246 (1979). doi:10.1029/JA084iA12p07239

I.V. Golovchanskaya, Y.P. Maltsev, On the identification of plasma sheet flapping waves observed by Cluster. Geophys. Res. Lett. 32, 2102 (2005). doi:10.1029/2004GL021552

M. Goossens, S. Poedts, D. Hermans, On the existence of the continuous spectrum of ideal MHD in a 2D magnetostatic equilibrium. Sol. Phys. 102, 51-66 (1985). doi:10.1007/BF00154037

M. Goossens, J.V. Hollweg, T. Sakurai, Resonant behaviour of MHD waves on magnetic flux tubes. III. Effect of equilibrium flow. Sol. Phys. 138, 233-255 (1992)

M. Goossens, I. Arregui, J.L. Ballester, T.J. Wang, Analytic approximate seismology of transversely oscillating coronal loops. Astron. Astrophys. 484, 851-857 (2008). doi:10.1051/0004-6361:200809728

M. Goossens, R. Erdélyi, M.S. Ruderman, Resonant MHD waves in the solar atmosphere. Space Sci. Rev. 158, 289-338 (2011). doi:10.1007/s11214-010-9702-7

M. Goossens, J. Andries, R. Soler, T. Van Doorsselaere, I. Arregui, J. Terradas, Surface Alfvén waves in solar flux tubes. Astrophys. J. 753, 111 (2012). doi:10.1088/0004-637X/753/2/111

M. Goossens, R. Soler, J. Terradas, T. Van Doorsselaere, G. Verth, The transverse and rotational motions of magnetohydrodynamic kink waves in the solar atmosphere. Astrophys. J. 788, 9 (2014). doi:10.1088/0004-637X/788/1/9

N. Gopalswamy, S. Yashiro, M. Temmer, J. Davila, W.T. Thompson, S. Jones, R.T.J. McAteer, J.-P. Wuelser, S. Freeland, R.A. Howard, EUV wave reflection from a coronal hole. Astrophys. J. Lett. 691, 123-127 (2009). doi:10.1088/0004-637X/691/2/L123 
P.C. Grigis, A.O. Benz, The evolution of reconnection along an arcade of magnetic loops. Astrophys. J. Lett. 625, 143-146 (2005). doi:10.1086/431147

M. Gruszecki, V.M. Nakariakov, T. van Doorsselaere, T.D. Arber, Phenomenon of Alfvénic vortex shedding. Phys. Rev. Lett. 105(5), 055004 (2010). doi:10.1103/PhysRevLett.105.055004

M. Gruszecki, V.M. Nakariakov, T. Van Doorsselaere, Intensity variations associated with fast sausage modes. Astron. Astrophys. 543, 12 (2012). doi:10.1051/0004-6361/201118168

A.V. Guglielmi, O.A. Pokhotelov, Geoelectromagnetic Waves (1996)

A.V. Gul'elmi, Annular trap for low-frequency wave in the earth's magnetosphere. Sov. Phys. JETP 12, 25-28 (1970)

X.C. Guo, C. Wang, Y.Q. Hu, Global MHD simulation of the Kelvin-Helmholtz instability at the magnetopause for northward interplanetary magnetic field. J. Geophys. Res. Space Phys. 115, 10218 (2010). doi:10.1029/2009JA015193

A. Hasegawa, Drift mirror instability of the magnetosphere. Phys. Fluids 12, 2642-2650 (1969). doi:10.1063/ 1.1692407

H. Hasegawa, M. Fujimoto, T.-D. Phan, H. Rème, A. Balogh, M.W. Dunlop, C. Hashimoto, R. TanDokoro, Transport of solar wind into Earth's magnetosphere through rolled-up Kelvin-Helmholtz vortices. Nature 430, 755-758 (2004). doi:10.1038/nature02799

D.H. Hathaway, The solar cycle. Living Rev. Sol. Phys. 7, 1 (2010). doi:10.12942/1rsp-2010-1

B. Heilig, H. Lühr, M. Rother, Comprehensive study of ULF upstream waves observed in the topside ionosphere by CHAMP and on the ground. Ann. Geophys. 25, 737-754 (2007). doi:10.5194/angeo-25737-2007

B. Heilig, S. Lotz, J. Verō, P. Sutcliffe, J. Reda, K. Pajunpää, T. Raita, Empirically modelled Pc3 activity based on solar wind parameters. Ann. Geophys. 28, 1703-1722 (2010). doi:10.5194/angeo-28-1703-2010

J. Hershaw, C. Foullon, V.M. Nakariakov, E. Verwichte, Damped large amplitude transverse oscillations in an EUV solar prominence, triggered by large-scale transient coronal waves. Astron. Astrophys. 531, 53 (2011). doi:10.1051/0004-6361/201116750

A. Hillier, R.J. Morton, R. Erdélyi, A statistical study of transverse oscillations in a quiescent prominence. Astrophys. J. Lett. 779, 16 (2013). doi:10.1088/2041-8205/779/2/L16

J.V. Hollweg, Resonances of coronal loops. Astrophys. J. 277, 392-403 (1984). doi:10.1086/161706

J.V. Hollweg, Resonant decay of global MHD modes at 'thick' interfaces. J. Geophys. Res. 95, 2319-2324 (1990). doi:10.1029/JA095iA03p02319

J.V. Hollweg, G. Yang, Resonance absorption of compressible magnetohydrodynamic waves at thin 'surfaces'. J. Geophys. Res. 93, 5423-5436 (1988)

V. Holzwarth, D. Schmitt, M. Schüssler, Flow instabilities of magnetic flux tubes. II. Longitudinal flow. Astron. Astrophys. 469, 11-17 (2007). doi:10.1051/0004-6361:20077269

A.W. Hood, M. Ruderman, D.J. Pascoe, I. De Moortel, J. Terradas, A.N. Wright, Damping of kink waves by mode coupling. I. Analytical treatment. Astron. Astrophys. 551, 39 (2013). doi:10.1051/0004-6361/ 201220617

W.J. Hughes, D.J. Southwood, The screening of micropulsation signals by the atmosphere and ionosphere. J. Geophys. Res. 81, 3234-3240 (1976). doi:10.1029/JA081i019p03234

A.R. Inglis, V.M. Nakariakov, A multi-periodic oscillatory event in a solar flare. Astron. Astrophys. 493, 259-266 (2009). doi:10.1051/0004-6361:200810473

A.R. Inglis, V.M. Nakariakov, V.F. Melnikov, Multi-wavelength spatially resolved analysis of quasiperiodic pulsations in a solar flare. Astron. Astrophys. 487, 1147-1153 (2008). doi:10.1051/0004-6361: 20079323

A.R. Inglis, T. Van Doorsselaere, C.S. Brady, V.M. Nakariakov, Characteristics of magnetoacoustic sausage modes. Astron. Astrophys. 503, 569-575 (2009). doi:10.1051/0004-6361/200912088

J.A. Ionson, Resonant absorption of Alfvénic surface waves and the heating of solar coronal loops. Astrophys. J. 226, 650-673 (1978). doi:10.1086/156648

P. Jelínek, M. Karlický, Magnetoacoustic waves in diagnostics of the flare current sheets. Astron. Astrophys. 537, 46 (2012). doi:10.1051/0004-6361/201117883

L.K. Jian, C.T. Russell, J.G. Luhmann, R.J. Strangeway, J.S. Leisner, A.B. Galvin, Ion cyclotron waves in the solar wind observed by STEREO near 1 AU. Astrophys. J. Lett. 701, 105-109 (2009). doi:10.1088/ 0004-637X/701/2/L105

P.S. Joarder, V.M. Nakariakov, B. Roberts, A manifestation of negative energy waves in the solar atmosphere. Sol. Phys. 176, 285-297 (1997). doi:10.1023/A:1004977928351

B.B. Kadomtsev, Collective Phenomena in Plasmas (Elsevier Science Limited, Amsterdam, 1982)

B.B. Kadomtsev, O.P. Pogutse, Plasma instability due to particle trapping in a toroidal geometry. Sov. Phys. JETP 24, 1172-1179 (1967)

M. Karlický, Cyclic magnetic field reconnection. Astrophys. J. Lett. 692, 72-75 (2009). doi:10.1088/ 0004-637X/692/2/L72 
M. Karlický, M. Bárta, Successive merging of plasmoids and fragmentation in a flare current sheet and their X-ray and radio signatures. Astrophys. J. 733, 107 (2011). doi:10.1088/0004-637X/733/2/107

M. Karlický, B. Kliem, Reconnection of a kinking flux rope triggering the ejection of a microwave and hard X-ray source I. Observations and interpretation. Sol. Phys. 266, 71-89 (2010). doi:10.1007/ s11207-010-9606-4

M. Karlický, M. Bárta, H. Mészárosová, P. Zlobec, Time scales of the slowly drifting pulsating structure observed during the April 12, 2001 flare. Astron. Astrophys. 432, 705-712 (2005). doi:10.1051/ 0004-6361:20041551

M. Karlický, M. Bárta, J. Rybák, Radio spectra generated during coalescence processes of plasmoids in a flare current sheet. Astron. Astrophys. 514, 28 (2010). doi:10.1051/0004-6361/200913547

M. Karlický, P. Jelínek, H. Mészárosová, Magnetoacoustic waves in the narrowband dm-spikes sources. Astron. Astrophys. 529, 96 (2011). doi:10.1051/0004-6361/201016171

M. Karlický, H. Mészárosová, P. Jelínek, Radio fiber bursts and fast magnetoacoustic wave trains. Astron. Astrophys. 550, 1 (2013). doi:10.1051/0004-6361/201220296

A.C. Katsiyannis, D.R. Williams, R.T.J. McAteer, P.T. Gallagher, F.P. Keenan, F. Murtagh, Eclipse observations of high-frequency oscillations in active region coronal loops. Astron. Astrophys. 406, 709-714 (2003). doi:10.1051/0004-6361:20030458

A. Keiling, K. Takahashi, Review of Pi2 Models. Space Sci. Rev. 161, 63-148 (2011). doi:10.1007/s11214011-9818-4

A. Keiling, J.R. Wygant, C. Cattell, W. Peria, G. Parks, M. Temerin, F.S. Mozer, C.T. Russell, C.A. Kletzing, Correlation of Alfvén wave Poynting flux in the plasma sheet at 4-7 $\mathrm{R}_{E}$ with ionospheric electron energy flux. J. Geophys. Res. Space Phys. 107, 1132 (2002). doi:10.1029/2001JA900140

A. Keiling, M. Fujimoto, H. Hasegawa, F. Honary, V. Sergeev, V.S. Semenov, H.U. Frey, O. Amm, H. Rème, I. Dandouras, E. Lucek, Association of Pi2 pulsations and pulsed reconnection: ground and Cluster observations in the tail lobe at $16 \mathrm{R}_{E}$. Ann. Geophys. 24, 3433-3449 (2006). doi:10.5194/ angeo-24-3433-2006

L. Kepko, H.E. Spence, Observations of discrete, global magnetospheric oscillations directly driven by solar wind density variations. J. Geophys. Res. Space Phys. 108, 1257 (2003). doi:10.1029/2002JA009676

M.L. Khodachenko, V.V. Zaitsev, A.G. Kislyakov, A.V. Stepanov, Equivalent electric circuit models of coronal magnetic loops and related oscillatory phenomena on the Sun. Space Sci. Rev. 149, 83-117 (2009). doi:10.1007/s11214-009-9538-1

S. Kim, V.M. Nakariakov, K. Shibasaki, Slow magnetoacoustic oscillations in the microwave emission of solar flares. Astrophys. J. Lett. 756, 36 (2012). doi:10.1088/2041-8205/756/2/L36

S. Kim, V.M. Nakariakov, K.-S. Cho, Vertical kink oscillation of a magnetic flux rope structure in the solar corona. Astrophys. J. Lett. 797, 22 (2014). doi:10.1088/2041-8205/797/2/L22

M.G. Kivelson, S.-H. Chen, The Magnetopause: Surface Waves and Instabilities and Their Possible Dynamical Consequences (Am. Geophys. Union, Washington, 1995), pp. 257-268. ISBN 9781118663974. doi:10.1029/GM090p0257

M.G. Kivelson, D.J. Southwood, Coupling of global magnetospheric MHD eigenmodes to field line resonances. J. Geophys. Res. 91, 4345-4351 (1986). doi:10.1029/JA091iA04p04345

B. Kliem, M. Karlický, A.O. Benz, Solar flare radio pulsations as a signature of dynamic magnetic reconnection. Astron. Astrophys. 360, 715-728 (2000)

D.Y. Klimushkin, Method of description of the Alfvén and magnetosonic branches of inhomogeneous plasma oscillations. Plasma Phys. Rep. 20, 280-286 (1994)

D.Y. Klimushkin, Resonators for hydromagnetic waves in the magnetosphere. J. Geophys. Res. 103, 23692376 (1998). doi:10.1029/97JA02193

D.Y. Klimushkin, The propagation of high- $m$ Alfvén waves in the Earth's magnetosphere and their interaction with high-energy particles. J. Geophys. Res. 105, 23303-23310 (2000). doi:10.1029/1999JA000396

D.Y. Klimushkin, How energetic particles construct and destroy poloidal high-m Alfvén waves in the magnetosphere. Planet. Space Sci. 55, 722-730 (2007). doi:10.1016/j.pss.2005.11.006

D.Y. Klimushkin, P.N. Mager, The structure of low-frequency standing Alfvén waves in the box model of the magnetosphere with magnetic field shear. J. Plasma Phys. 70, 379-395 (2004). doi:10.1017/ S0022377803002563

D.Y. Klimushkin, P.N. Mager, Spatial structure and stability of coupled Alfvén and drift compressional modes in non-uniform magnetosphere: gyrokinetic treatment. Planet. Space Sci. 59, 1613-1620 (2011). doi:10.1016/j.pss.2011.07.010

D.Y. Klimushkin, P.N. Mager, The Alfvén wave parallel electric field in non-uniform space plasmas. Astrophys. Space Sci. 350, 579-583 (2014). doi:10.1007/s10509-013-1774-X

D. Klimushkin, P. Mager, K. Glassmeier, Toroidal and poloidal Alfvén waves with arbitrary azimuthal wavenumbers in a finite pressure plasma in the Earth's magnetosphere. Ann. Geophys. 22, 267-287 (2004). doi:10.5194/angeo-22-267-2004 
D.Y. Klimushkin, P.N. Mager, V.A. Pilipenko, On the ballooning instability of the coupled Alfvén and drift compressional modes. Earth Planets Space 64, 777-781 (2012). doi:10.5047/eps.2012.04.002

K. Kobayashi, J. Cirtain, A.R. Winebarger, K. Korreck, L. Golub, R.W. Walsh, B. De Pontieu, C. DeForest, A. Title, S. Kuzin, S. Savage, D. Beabout, B. Beabout, W. Podgorski, D. Caldwell, K. McCracken, M. Ordway, H. Bergner, R. Gates, S. McKillop, P. Cheimets, S. Platt, N. Mitchell, D. Windt, The highresolution coronal imager (Hi-C). Sol. Phys. 289, 4393-4412 (2014). doi:10.1007/s11207-014-0544-4

M.M. Kobrin, V.I. Malygin, S.D. Snegirev, Long-period pulsations of the earth's magnetic field with periods more than 20 minutes before proton flares on the sun. Planet. Space Sci. 33, 1251-1257 (1985). doi:10.1016/0032-0633(85)90003-0

D.Y. Kolotkov, V.M. Nakariakov, E.G. Kupriyanova, H. Ratcliffe, K. Shibasaki, Multi-mode quasi-periodic pulsations in a solar flare. Astron. Astrophys. 574, 53 (2015). doi:10.1051/0004-6361/201424988

Y.G. Kopylova, A.V. Melnikov, A.V. Stepanov, Y.T. Tsap, T.B. Goldvarg, Oscillations of coronal loops and second pulsations of solar radio emission. Astron. Lett. 33, 706-713 (2007). doi:10.1134/ S1063773707100088

D.B. Korovinskiy, A. Divin, N.V. Erkaev, V.V. Ivanova, I.B. Ivanov, V.S. Semenov, G. Lapenta, S. Markidis, H.K. Biernat, M. Zellinger, MHD modeling of the double-gradient (kink) magnetic instability. J. Geophys. Res. 118, 1146-1158 (2013). doi:10.1002/jgra.50206

D.B. Korovinskiy, A.V. Divin, N.V. Erkaev, V.S. Semenov, A.V. Artemyev, V.V. Ivanova, I.B. Ivanov, G. Lapenta, S. Markidis, H.K. Biernat, The double-gradient magnetic instability: stabilizing effect of the guide field. Phys. Plasmas 22(1), 012904 (2015). doi:10.1063/1.4905706

D.A. Kozlov, A.S. Leonovich, Polarization splitting of the Alfvén wave spectrum in a dipole magnetosphere with a rotating plasma. Plasma Phys. Rep. 32, 765-774 (2006). doi:10.1134/S1063780X06090078

S. Krishna Prasad, D. Banerjee, T. Van Doorsselaere, J. Singh, Omnipresent long-period intensity oscillations in open coronal structures. Astron. Astrophys. 546, 50 (2012). doi:10.1051/0004-6361/201219885

S. Krishna Prasad, D. Banerjee, T. Van Doorsselaere, Frequency-dependent damping in propagating slow magneto-acoustic waves. Astrophys. J. 789, 118 (2014). doi:10.1088/0004-637X/789/2/118

E.G. Kupriyanova, V.F. Melnikov, V.M. Nakariakov, K. Shibasaki, Types of microwave quasi-periodic pulsations in single flaring loops. Sol. Phys. 267, 329-342 (2010). doi:10.1007/s11207-010-9642-0

E.G. Kupriyanova, V.F. Melnikov, K. Shibasaki, Evolution of the source of quasi-periodic microwave pulsations in a single flaring loop. Publ. Astron. Soc. Jpn. 65, 3 (2013). doi:10.1093/pasj/65.sp1.S3

N.A. Kurazhkovskaya, B.I. Klain, Effect of the solar wind and IMF parameters on the formation of long-period irregular pulsation burst regimes. Geomagn. Aeron. 52, 456-466 (2012). doi:10.1134/ S001679321204010X

R.-Y. Kwon, L. Ofman, O. Olmedo, M. Kramar, J.M. Davila, B.J. Thompson, K.-S. Cho, STEREO observations of fast magnetosonic waves in the extended solar corona associated with EIT/EUV waves. Astrophys. J. 766, 55 (2013). doi:10.1088/0004-637X/766/1/55

N. Labrosse, P. Heinzel, J.-C. Vial, T. Kucera, S. Parenti, S. Gunár, B. Schmieder, G. Kilper, Physics of solar prominences: spectral diagnostics and non-LTE modelling. Space Sci. Rev. 151, 243-332 (2010). doi:10.1007/s11214-010-9630-6

O. Le Contel, R. Pellat, A. Roux, Self-consistent quasi-static radial transport during the substorm growth phase. J. Geophys. Res. 105, 12929-12944 (2000). doi:10.1029/1999JA900498

D.-H. Lee, K. Kim, Compressional MHD waves in the magnetosphere: a new approach. J. Geophys. Res. 104, 12379-12386 (1999). doi:10.1029/1999JA900053

L.C. Lee, R.K. Albano, J.R. Kan, Kelvin-Helmholtz instability in the magnetopause-boundary layer region. J. Geophys. Res. 86, 54-58 (1981). doi:10.1029/JA086iA01p00054

L.C. Lee, Y. Shi, L.J. Lanzerotti, A mechanism for the generation of cusp region hydromagnetic waves. J. Geophys. Res. Space Phys. 93(A7), 7578-7585 (1988). doi:10.1029/JA093iA07p07578

J. Lemaire, Plasmoid motion across a tangential discontinuity-with application to the magnetopause. J. Plasma Phys. 33, 425-436 (1985). doi:10.1017/S0022377800002592

J.R. Lemen, A.M. Title, D.J. Akin, P.F. Boerner, C. Chou, J.F. Drake, D.W. Duncan, C.G. Edwards, F.M. Friedlaender, G.F. Heyman, N.E. Hurlburt, N.L. Katz, G.D. Kushner, M. Levay, R.W. Lindgren, D.P. Mathur, E.L. McFeaters, S. Mitchell, R.A. Rehse, C.J. Schrijver, L.A. Springer, R.A. Stern, T.D. Tarbell, J.-P. Wuelser, C.J. Wolfson, C. Yanari, J.A. Bookbinder, P.N. Cheimets, D. Caldwell, E.E. Deluca, R. Gates, L. Golub, S. Park, W.A. Podgorski, R.I. Bush, P.H. Scherrer, M.A. Gummin, P. Smith, G. Auker, P. Jerram, P. Pool, R. Soufli, D.L. Windt, S. Beardsley, M. Clapp, J. Lang, N. Waltham, The Atmospheric Imaging Assembly (AIA) on the Solar Dynamics Observatory (SDO). Sol. Phys. 275, 17-40 (2012). doi:10.1007/s11207-011-9776-8

A.S. Leonovich, V.A. Mazur, Linear transformation of the standing Alfvén wave in an axisymmetric magnetosphere. Planet. Space Sci. 43, 885-893 (1995a). doi:10.1016/0032-0633(94)00207-8

A.S. Leonovich, V.A. Mazur, Magnetospheric resonator for transverse-small-scale standing Alfvén waves. Planet. Space Sci. 43, 881-883 (1995b). doi:10.1016/0032-0633(94)00206-7 
A.S. Leonovich, V.A. Mazur, On the spectrum of magnetosonic eigenoscillations of an axisymmetric magnetosphere. J. Geophys. Res. 106, 3919-3928 (2001). doi:10.1029/2000JA000228

A.S. Leonovich, V.A. Mazur, Eigen ultra-low-frequency magnetosonic oscillations of the near plasma sheet. Cosm. Res. 46, 327-334 (2008). doi:10.1134/S0010952508040072

A.S. Leonovich, V.V. Mishin, J.B. Cao, Penetration of magnetosonic waves into the magnetosphere: influence of a transition layer. Ann. Geophys. 21, 1083-1093 (2003). doi:10.5194/angeo-21-1083-2003

A.S. Leonovich, D.A. Kozlov, V.A. Pilipenko, Magnetosonic resonance in a dipole-like magnetosphere. Ann. Geophys. 24, 2277-2289 (2006). doi:10.5194/angeo-24-2277-2006

B. Leroy, Propagation of waves in an atmosphere in the presence of a magnetic field. II. The reflection of Alfvén waves. Astron. Astrophys. 91, 136-146 (1980)

M.R. Lessard, D.J. Knudsen, Ionospheric reflection of small-scale Alfvén waves. Geophys. Res. Lett. 28, 3573-3576 (2001). doi:10.1029/2000GL012529

W.Y. Li, X.C. Guo, C. Wang, Spatial distribution of Kelvin-Helmholtz instability at low-latitude boundary layer under different solar wind speed conditions. J. Geophys. Res. Space Phys. 117, 8230 (2012). doi:10.1029/2012JA017780

Y. Lin, Filament thread-like structures and their small-amplitude oscillations. Space Sci. Rev. 158, 237-266 (2011). doi:10.1007/s11214-010-9672-9

Y. Lin, R. Soler, O. Engvold, J.L. Ballester, Ø. Langangen, R. Oliver, L.H.M. Rouppe van der Voort, Swaying threads of a solar filament. Astrophys. J. 704, 870-876 (2009). doi:10.1088/0004-637X/704/1/870

W. Liu, L. Ofman, Advances in observing various coronal EUV waves in the SDO Era and their seismological applications (Invited Review). Sol. Phys. 289, 3233-3277 (2014). doi:10.1007/s11207-014-0528-4

W. Liu, A.M. Title, J. Zhao, L. Ofman, C.J. Schrijver, M.J. Aschwanden, B. De Pontieu, T.D. Tarbell, Direct imaging of quasi-periodic fast propagating waves of $\sim 2000 \mathrm{~km} \mathrm{~s}^{-1}$ in the low solar corona by the solar dynamics observatory atmospheric imaging assembly. Astrophys. J. Lett. 736, 13 (2011). doi:10.1088/2041-8205/736/1/L13

D.M. Long, P.T. Gallagher, R.T.J. McAteer, D.S. Bloomfield, Deceleration and dispersion of large-scale coronal bright fronts. Astron. Astrophys. 531, 42 (2011). doi:10.1051/0004-6361/201015879

N.F. Loureiro, A.A. Schekochihin, S.C. Cowley, Instability of current sheets and formation of plasmoid chains. Phys. Plasmas 14(10), 100703 (2007). doi:10.1063/1.2783986

V.I. Lukovnikova, V.A. Parkhomov, Geomagnetic pulsations associated with chromospheric flares. Geofiz. ž. 6, 52-58 (1984)

P.M. Lushnikov, Two mechanisms of surface wave generation: Kelvin-Helmholtz and Miles instabilities. Atmos. Ocean. Phys. 34, 370-377 (1998)

R.L. Lysak, Feedback instability of the ionospheric resonant cavity. J. Geophys. Res. 96, 1553-1568 (1991). doi:10.1029/90JA02154

R.L. Lysak, C.T. Dum, Dynamics of magnetosphere-ionosphere coupling including turbulent transport. J. Geophys. Res. Space Phys. 88, 365-380 (1983a). doi:10.1029/JA088iA01p00365

R.L. Lysak, C.T. Dum, Dynamics of magnetosphere-ionosphere coupling including turbulent transport. J. Geophys. Res. 88, 365-380 (1983b). doi:10.1029/JA088iA01p00365

R.L. Lysak, D.-H. Lee, Response of the dipole magnetosphere to pressure pulses. Geophys. Res. Lett. 19, 937-940 (1992). doi:10.1029/92GL00625

P.N. Mager, D.Y. Klimushkin, Theory of azimuthally small-scale Alfvén waves in an axisymmetric magnetosphere with small but finite plasma pressure. J. Geophys. Res. Space Phys. 107, 1356 (2002). doi:10.1029/2001JA009137

P.N. Mager, D.Y. Klimushkin, Spatial localization and azimuthal wave numbers of Alfvén waves generated by drift-bounce resonance in the magnetosphere. Ann. Geophys. 23, 3775-3784 (2005). doi:10.5194/ angeo-23-3775-2005

P.N. Mager, D.Y. Klimushkin, Alfvén ship waves: high-m ULF pulsations in the magnetosphere generated by a moving plasma inhomogeneity. Ann. Geophys. 26, 1653-1663 (2008). doi:10.5194/ angeo-26-1653-2008

P.N. Mager, Y.D. Klimushkin, Giant pulsations as modes of a transverse Alfvénic resonator on the plasmapause. Earth Planets Space 65, 397-409 (2013). doi:10.5047/eps.2012.10.002

P.N. Mager, D.Y. Klimushkin, V.A. Pilipenko, S. Schäfer, Field-aligned structure of poloidal Alfvén waves in a finite pressure plasma. Ann. Geophys. 27, 3875-3882 (2009). doi:10.5194/angeo-27-3875-2009

P.N. Mager, D.Y. Klimushkin, D.V. Kostarev, Drift-compressional modes generated by inverted plasma distributions in the magnetosphere. J. Geophys. Res. Space Phys. 118, 4915-4923 (2013). doi:10.1002/ jgra.50471

A. Malanushenko, C.J. Schrijver, On the anisotropy in expansion of magnetic flux tubes in the solar corona. Astrophys. J. 775, 120 (2013). doi:10.1088/0004-637X/775/2/120 
J. Martínez-Sykora, B. De Pontieu, V. Hansteen, Two-dimensional radiative magnetohydrodynamic simulations of the importance of partial ionization in the chromosphere. Astrophys. J. 753, 161 (2012). doi:10.1088/0004-637X/753/2/161

M. Mathioudakis, D.B. Jess, R. Erdélyi, Alfvén waves in the solar atmosphere. From theory to observations. Space Sci. Rev. 175, 1-27 (2013). doi:10.1007/s11214-012-9944-7

V.A. Mazur, D.A. Chuiko, Azimuthal inhomogeneity in the MHD waveguide in the outer magnetosphere. J. Geophys. Res. Space Phys. 120, 4641-4655 (2015). doi:10.1002/2014JA020819

V.A. Mazur, A.S. Leonovich, ULF hydromagnetic oscillations with the discrete spectrum as eigenmodes of MHD-resonator in the near-Earth part of the plasma sheet. Ann. Geophys. 24, 1639-1648 (2006). doi:10.5194/angeo-24-1639-2006

N.G. Mazur, E.N. Fedorov, V.A. Pilipenko, On the possibility of reflection of Alfvén waves in a curvilinear magnetic field. Plasma Phys. Rep. 30, 413-421 (2004)

S.W. McIntosh, B. de Pontieu, M. Carlsson, V. Hansteen, P. Boerner, M. Goossens, Alfvénic waves with sufficient energy to power the quiet solar corona and fast solar wind. Nature 475, 477-480 (2011). doi:10.1038/nature 10235

J.A. McLaughlin, A.W. Hood, MHD wave propagation in the neighbourhood of a two-dimensional null point. Astron. Astrophys. 420, 1129-1140 (2004). doi:10.1051/0004-6361:20035900

J.A. McLaughlin, L. Ofman, Three-dimensional magnetohydrodynamic wave behavior in active regions: individual loop density structure. Astrophys. J. 682, 1338-1350 (2008). doi:10.1086/588799

J.A. McLaughlin, J.O. Thurgood, D. MacTaggart, On the periodicity of oscillatory reconnection. Astron. Astrophys. 548, 98 (2012). doi:10.1051/0004-6361/201220234

V.F. Melnikov, V.E. Reznikova, K. Shibasaki, V.M. Nakariakov, Spatially resolved microwave pulsations of a flare loop. Astron. Astrophys. 439, 727-736 (2005). doi:10.1051/0004-6361:20052774

V.F. Melnikov, D.E. Gary, G.M. Nita, Peak frequency dynamics in solar microwave bursts. Sol. Phys. 253, 43-73 (2008). doi:10.1007/s11207-008-9275-8

F.W. Menk, C.L. Waters, Magnetoseismology. Ground-Based Remote Sensing of Earth's Magnetosphere (VCH, Weinheim, 2013), p. 251. 978-3-527-41027-9

V.G. Merkin, J.G. Lyon, S.G. Claudepierre, Kelvin-Helmholtz instability of the magnetospheric boundary in a three-dimensional global MHD simulation during northward IMF conditions. J. Geophys. Res. Space Phys. 118, 5478-5496 (2013). doi:10.1002/jgra.50520

H. Mészárosová, M. Karlický, J. Rybák, K. Jiřička, Tadpoles in wavelet spectra of a solar decimetric radio burst. Astrophys. J. Lett. 697, 108-110 (2009). doi:10.1088/0004-637X/697/2/L108

H. Mészárosová, M. Karlický, P. Jelínek, J. Rybák, Magnetoacoustic waves propagating along a dense slab and Harris current sheet and their wavelet spectra. Astrophys. J. 788, 44 (2014). doi:10.1088/0004$637 X / 788 / 1 / 44$

V.V. Mishin, Velocity boundary layers in the distant geotail and the Kelvin Helmholtz instability. Planet. Space Sci. 53, 157-160 (2005). doi:10.1016/j.pss.2004.09.040

A.P. Mitra (ed.), in Ionospheric Effects of Solar Flares. Astrophysics and Space Science Library, vol. 46 (1974)

A. Miura, P.L. Pritchett, Nonlocal stability analysis of the MHD Kelvin-Helmholtz instability in a compressible plasma. J. Geophys. Res. 87, 7431-7444 (1982). doi:10.1029/JA087iA09p07431

G.E. Moreton, $\mathrm{H} \alpha$ observations of flare-initiated disturbances with velocities $\sim 1000 \mathrm{~km} / \mathrm{sec}$. Astron. J. 65, 494 (1960). doi:10.1086/108346

R.J. Morton, J.A. McLaughlin, Hi-C and AIA observations of transverse magnetohydrodynamic waves in active regions. Astron. Astrophys. 553, 10 (2013). doi:10.1051/0004-6361/201321465

R.J. Morton, G. Verth, J.A. McLaughlin, R. Erdélyi, Determination of sub-resolution structure of a jet by solar magnetoseismology. Astrophys. J. 744, 5 (2012). doi:10.1088/0004-637X/744/1/5

G. Mossessian, G.D. Fleishman, Modeling of gyrosynchrotron radio emission pulsations produced by magnetohydrodynamic loop oscillations in solar flares. Astrophys. J. 748, 140 (2012). doi:10.1088/0004-637X/ $748 / 2 / 140$

D.A.N. Müller, H. Peter, V.H. Hansteen, Dynamics of solar coronal loops. II. Catastrophic cooling and highspeed downflows. Astron. Astrophys. 424, 289-300 (2004). doi:10.1051/0004-6361:20040403

V.M. Nakariakov, Magnetohydrodynamic waves in coronal polar plumes. Philos. Trans. R. Soc. Lond. Ser. A 364, 473-483 (2006). doi:10.1098/rsta.2005.1711

V.M. Nakariakov, V.F. Melnikov, Modulation of gyrosynchrotron emission in solar and stellar flares by slow magnetoacoustic oscillations. Astron. Astrophys. 446, 1151-1156 (2006). doi:10.1051/ 0004-6361:20053944

V.M. Nakariakov, V.F. Melnikov, Quasi-periodic pulsations in solar flares. Space Sci. Rev. 149, 119-151 (2009). doi:10.1007/s11214-009-9536-3

V.M. Nakariakov, E. Verwichte, Coronal waves and oscillations. Living Rev. Sol. Phys. 2, 3 (2005). doi:10. 12942/lrsp-2005-3 
V.M. Nakariakov, I.V. Zimovets, Slow magnetoacoustic waves in two-ribbon flares. Astrophys. J. Lett. 730, 27 (2011). doi:10.1088/2041-8205/730/2/L27

V.M. Nakariakov, L. Ofman, E.E. Deluca, B. Roberts, J.M. Davila, TRACE observation of damped coronal loop oscillations: implications for coronal heating. Science 285, 862-864 (1999). doi:10.1126/science.285.5429.862

V.M. Nakariakov, E. Verwichte, D. Berghmans, E. Robbrecht, Slow magnetoacoustic waves in coronal loops. Astron. Astrophys. 362, 1151-1157 (2000)

V.M. Nakariakov, V.F. Melnikov, V.E. Reznikova, Global sausage modes of coronal loops. Astron. Astrophys. 412, 7-10 (2003). doi:10.1051/0004-6361:20031660

V.M. Nakariakov, D. Tsiklauri, A. Kelly, T.D. Arber, M.J. Aschwanden, Acoustic oscillations in solar and stellar flaring loops. Astron. Astrophys. 414, 25-28 (2004a). doi:10.1051/0004-6361:20031738

V.M. Nakariakov, T.D. Arber, C.E. Ault, A.C. Katsiyannis, D.R. Williams, F.P. Keenan, Time signatures of impulsively generated coronal fast wave trains. Mon. Not. R. Astron. Soc. 349, 705-709 (2004b). doi:10.1111/j.1365-2966.2004.07537.x

V.M. Nakariakov, D.J. Pascoe, T.D. Arber, Short quasi-periodic MHD waves in coronal structures. Space Sci. Rev. 121, 115-125 (2005). doi:10.1007/s11214-006-4718-8

V.M. Nakariakov, C. Foullon, E. Verwichte, N.P. Young, Quasi-periodic modulation of solar and stellar flaring emission by magnetohydrodynamic oscillations in a nearby loop. Astron. Astrophys. 452, 343-346 (2006). doi:10.1051/0004-6361:20054608

V.M. Nakariakov, M.J. Aschwanden, T. van Doorsselaere, The possible role of vortex shedding in the excitation of kink-mode oscillations in the solar corona. Astron. Astrophys. 502, 661-664 (2009). doi:10.1051/0004-6361/200810847

V.M. Nakariakov, A.R. Inglis, I.V. Zimovets, C. Foullon, E. Verwichte, R. Sych, I.N. Myagkova, Oscillatory processes in solar flares. Plasma Phys. Control. Fusion 52(12), 124009 (2010a). doi:10.1088/0741-3335/ $52 / 12 / 124009$

V.M. Nakariakov, C. Foullon, I.N. Myagkova, A.R. Inglis, Quasi-periodic pulsations in the gamma-ray emission of a solar flare. Astrophys. J. Lett. 708, 47-51 (2010b). doi:10.1088/2041-8205/708/1/L47

V.M. Nakariakov, C. Hornsey, V.F. Melnikov, Sausage oscillations of coronal plasma structures. Astrophys. J. 761, 134 (2012). doi:10.1088/0004-637X/761/2/134

P.H. Ng, V.L. Patel, S. Chen, Drift compressional instability in the magnetosphere. J. Geophys. Res. 89, 10763-10769 (1984). doi:10.1029/JA089iA12p10763

Z. Ning, Imaging observations of X-ray quasi-periodic oscillations at 3-6 keV in the 26 December 2002 solar flare. Sol. Phys. 289, 1239-1256 (2014). doi:10.1007/s11207-013-0405-6

M.N. Nishino, M. Fujimoto, G. Ueno, T. Mukai, Y. Saito, Origin of temperature anisotropies in the cold plasma sheet: geotail observations around the Kelvin-Helmholtz vortices. Ann. Geophys. 25, 20692086 (2007). doi:10.5194/angeo-25-2069-2007

G. Nisticò, V.M. Nakariakov, E. Verwichte, Decaying and decayless transverse oscillations of a coronal loop. Astron. Astrophys. 552, 57 (2013). doi:10.1051/0004-6361/201220676

G. Nisticò, D.J. Pascoe, V.M. Nakariakov, Observation of a high-quality quasi-periodic rapidly propagating wave train using SDO/AIA. Astron. Astrophys. 569, 12 (2014). doi:10.1051/0004-6361/201423763

L. Ofman, Chromospheric leakage of Alfvén waves in coronal loops. Astrophys. J. Lett. 568, 135-138 (2002). doi: $10.1086 / 340329$

L. Ofman, Progress, challenges, and perspectives of the 3D MHD numerical modeling of oscillations in the solar corona. Space Sci. Rev. 149, 153-174 (2009a). doi:10.1007/s11214-009-9501-1

L. Ofman, Three-dimensional magnetohydrodynamic models of twisted multithreaded coronal loop oscillations. Astrophys. J. 694, 502-511 (2009b). doi:10.1088/0004-637X/694/1/502

L. Ofman, Wave modeling of the solar wind. Living Rev. Sol. Phys. 7, 4 (2010). doi:10.12942/lrsp-2010-4

L. Ofman, M.J. Aschwanden, Damping time scaling of coronal loop oscillations deduced from transition region and coronal explorer observations. Astrophys. J. Lett. 576, 153-156 (2002). doi:10.1086/343886

L. Ofman, L. Sui, Oscillations of hard X-ray flare emission observed by RHESSI: effects of super-Alfvénic beams? Astrophys. J. Lett. 644, 149-152 (2006). doi:10.1086/505622

L. Ofman, B.J. Thompson, Interaction of EIT waves with coronal active regions. Astrophys. J. 574, 440-452 (2002). doi:10.1086/340924

L. Ofman, B.J. Thompson, SDO/AIA observation of Kelvin-Helmholtz instability in the solar corona. Astrophys. J. Lett. 734, 11 (2011). doi:10.1088/2041-8205/734/1/L11

L. Ofman, T. Wang, Hot coronal loop oscillations observed by SUMER: slow magnetosonic wave damping by thermal conduction. Astrophys. J. Lett. 580, 85-88 (2002). doi:10.1086/345548

L. Ofman, J.M. Davila, R.S. Steinolfson, Coronal heating by the resonant absorption of Alfvén waves: the effect of viscous stress tensor. Astrophys. J. 421, 360-371 (1994). doi:10.1086/173654

L. Ofman, J.M. Davila, R.S. Steinolfson, Coronal heating by the resonant absorption of Alfvén waves: wavenumber scaling laws. Astrophys. J. 444, 471-477 (1995). doi:10.1086/175621 
L. Ofman, M. Romoli, G. Poletto, G. Noci, J.L. Kohl, Ultraviolet coronagraph spectrometer observations of density fluctuations in the solar wind. Astrophys. J. Lett. 491, 111-114 (1997). doi:10.1086/311067

L. Ofman, V.M. Nakariakov, C.E. DeForest, Slow magnetosonic waves in coronal plumes. Astrophys. J. 514, 441-447 (1999). doi:10.1086/306944

L. Ofman, V.M. Nakariakov, N. Sehgal, Dissipation of slow magnetosonic waves in coronal plumes. Astrophys. J. 533, 1071-1083 (2000). doi:10.1086/308691

L. Ofman, W. Liu, A. Title, M. Aschwanden, Modeling super-fast magnetosonic waves observed by SDO in active region funnels. Astrophys. J. Lett. 740, 33 (2011). doi:10.1088/2041-8205/740/2/L33

L. Ofman, T.J. Wang, J.M. Davila, Slow magnetosonic waves and fast flows in active region loops. Astrophys. J. 754, 111 (2012). doi:10.1088/0004-637X/754/2/111

R. Oliver, J.L. Ballester, Oscillations in quiescent solar prominences observations and theory (Invited Review). Sol. Phys. 206, 45-67 (2002). doi:10.1023/A:1014915428440

A.A. Ostapenko, S.V. Poliakov, Dynamics of the coefficient of reflection of Alfvén waves in the Pc1 range from the ionosphere during variations of the electron density of the lower ionosphere. Geomagn. Aeron. 30, 50-56 (1990)

S. Parenti, Solar prominences: observations. Living Rev. Sol. Phys. 11, 1 (2014). doi:10.12942/lrsp-2014-1

V.A. Parkhomov, A.V. Moldavanov, B. Tsegmed, On two different geomagnetic manifestations of solar flare November 4, 2003. J. Atmos. Sol.-Terr. Phys. 68, 1370-1382 (2006). doi:10.1016/j.jastp.2006.05.002

V.A. Parkhomov, A.V. Dmitriev, A.V. Moldavanov, Unusual sudden ionospheric disturbance from solar flare of 4 November 2003. J. Atmos. Sol.-Terr. Phys. 70, 1963-1970 (2008). doi:10.1016/j.jastp.2008.03.011

D.J. Pascoe, V.M. Nakariakov, E.G. Kupriyanova, Fast magnetoacoustic wave trains in magnetic funnels of the solar corona. Astron. Astrophys. 560, 97 (2013a). doi:10.1051/0004-6361/201322678

D.J. Pascoe, A.W. Hood, I. De Moortel, A.N. Wright, Damping of kink waves by mode coupling. II. Parametric study and seismology. Astron. Astrophys. 551, 40 (2013b). doi:10.1051/0004-6361/201220620

S. Patsourakos, A. Vourlidas, "Extreme Ultraviolet Waves" are waves: first quadrature observations of an extreme ultraviolet wave from STEREO. Astrophys. J. Lett. 700, 182-186 (2009). doi:10.1088/ 0004-637X/700/2/L182

S. Patsourakos, A. Vourlidas, On the nature and genesis of EUV waves: a synthesis of observations from SOHO, STEREO, SDO, and Hinode (Invited Review). Sol. Phys. 281, 187-222 (2012). doi:10.1007/ s11207-012-9988-6

H. Peter, S. Bingert, Constant cross section of loops in the solar corona. Astron. Astrophys. 548, 1 (2012). doi:10.1051/0004-6361/201219473

A. Petrosyan, A. Balogh, M.L. Goldstein, J. Léorat, E. Marsch, K. Petrovay, B. Roberts, R. von Steiger, J.C. Vial, Turbulence in the solar atmosphere and solar wind. Space Sci. Rev. 156, 135-238 (2010). doi:10.1007/s11214-010-9694-3

V.A. Pilipenko, E.N. Fedorov, M.J. Engebretson, Alfvén resonator in the topside ionosphere beneath the auroral acceleration region. J. Geophys. Res. 107(A9) (2002). doi:10.1029/2002JA009282

V.A. Pilipenko, N.G. Mazur, E.N. Fedorov, M.J. Engebretson, Interaction of propagating magnetosonic and Alfvén waves in a longitudinally inhomogeneous plasma. J. Geophys. Res. 113, 8218 (2008). doi:10.1029/2007JA012651

F. Plaschke, V. Angelopoulos, K.-H. Glassmeier, Magnetopause surface waves: THEMIS observations compared to MHD theory. J. Geophys. Res. Space Phys. 118, 1483-1499 (2013). doi:10.1002/jgra.50147

J.J. Podesta, Evidence of kinetic Alfvén waves in the solar wind at 1 AU. Sol. Phys. 286, 529-548 (2013). doi:10.1007/s11207-013-0258-z

S. Poedts, M. Goossens, W. Kerner, Numerical simulation of coronal heating by resonant absorption of Alfvén waves. Sol. Phys. 123, 83-115 (1989). doi:10.1007/BF00150014

S. Poedts, M. Goossens, W. Kerner, On the efficiency of coronal loop heating by resonant absorption. Astrophys. J. 360, 279-287 (1990). doi:10.1086/169118

O.A. Pokhotelov, M.A. Balikhin, H.S.-C.K. Alleyne, O.G. Onishchenko, Mirror instability with finite electron temperature effects. J. Geophys. Res. 105, 2393-2402 (2000). doi:10.1029/1999JA900351

O.A. Pokhotelov, V. Khruschev, M. Parrot, S. Senchenkov, V.P. Pavlenko, Ionospheric Alfvén resonator revisited: feedback instability. J. Geophys. Res. 106, 25813-25824 (2001). doi:10.1029/2000JA000450

P. Porazik, Z. Lin, Gyrokinetic particle simulation of drift-compressional modes in dipole geometry. Phys. Plasmas 18(7), 072107 (2011). doi:10.1063/1.3605031

A.S. Potapov, ULF wave activity in high-speed streams of the solar wind: impact on the magnetosphere. J. Geophys. Res. Space Phys. 118, 6465-6477 (2013). doi:10.1002/2013JA019119

A.S. Potapov, T.N. Polyushkina, V.A. Pulyaev, Observations of ULF waves in the solar corona and in the solar wind at the Earth's orbit. J. Atmos. Sol.-Terr. Phys. 102, 235-242 (2013). doi:10.1016/ j.jastp.2013.06.001 
I.A. Price, C.L. Waters, F.W. Menk, G.J. Bailey, B.J. Fraser, A technique to investigate plasma mass density in the topside ionosphere using ULF waves. J. Geophys. Res. 104, 12723-12732 (1999). doi:10.1029/1999JA900042

Z.-y. Pu, M.G. Kivelson, Kelvin-Helmholtz instability at the magnetopause: energy flux into the magnetosphere. J. Geophys. Res. 88, 853-862 (1983). doi:10.1029/JA088iA02p00853

I.J. Rae, I.R. Mann, C.E.J. Watt, L.M. Kistler, W. Baumjohann, Equator-S observations of drift mirror mode waves in the dawnside magnetosphere. J. Geophys. Res. 112, 11203 (2007). doi:10.1029/ 2006JA012064

I.J. Rae, K.R. Murphy, C.E.J. Watt, G. Rostoker, R. Rankin, I.R. Mann, C.R. Hodgson, H.U. Frey, A.W. Degeling, C. Forsyth, Field line resonances as a trigger and a tracer for substorm onset. J. Geophys. Res. Space Phys. 119, 5343-5363 (2014). doi:10.1002/2013JA018889

F. Reale, Coronal loops: observations and modeling of confined plasma. Living Rev. Sol. Phys. 11, 4 (2014). doi:10.12942/lrsp-2014-4

H.A.S. Reid, N. Vilmer, E.P. Kontar, Characteristics of the flare acceleration region derived from simultaneous hard X-ray and radio observations. Astron. Astrophys. 529, 66 (2011). doi:10.1051/ 0004-6361/201016181

V.E. Reznikova, P. Antolin, T. Van Doorsselaere, Forward modeling of gyrosynchrotron intensity perturbations by sausage modes. Astrophys. J. 785, 86 (2014). doi:10.1088/0004-637X/785/2/86

E. Robbrecht, E. Verwichte, D. Berghmans, J.F. Hochedez, S. Poedts, V.M. Nakariakov, Slow magnetoacoustic waves in coronal loops: EIT and TRACE. Astron. Astrophys. 370, 591-601 (2001). doi:10.1051/0004-6361:20010226

B. Roberts, Slow magnetohydrodynamic waves in the solar atmosphere. Philos. Trans. R. Soc. Lond. Ser. A 364, 447-460 (2006). doi:10.1098/rsta.2005.1709

B. Roberts, P.M. Edwin, A.O. Benz, Fast pulsations in the solar corona. Nature 305, 688-690 (1983). doi: $10.1038 / 305688 \mathrm{a} 0$

M.S. Ruderman, B. Roberts, The damping of coronal loop oscillations. Astrophys. J. 577, 475-486 (2002)

C.T. Russell, R.C. Elphic, ISEE observations of flux transfer events at the dayside magnetopause. Geophys. Res. Lett. 6, 33-36 (1979). doi:10.1029/GL006i001p00033

A.J.B. Russell, L. Fletcher, Propagation of Alfvénic waves from corona to chromosphere and consequences for solar flares. Astrophys. J. 765, 81 (2013). doi:10.1088/0004-637X/765/2/81

T. Sakurai, M. Goossens, J.V. Hollweg, Resonant behaviour of MHD waves on magnetic flux tubes. I. Connection formulae at the resonant surfaces. Sol. Phys. 133, 227-245 (1991)

J.C. Samson, B.G. Harrold, J.M. Ruohoniemi, R.A. Greenwald, A.D.M. Walker, Field line resonances associated with MHD waveguides in the magnetosphere. Geophys. Res. Lett. 19, 441-444 (1992). doi:10.1029/92GL00116

S. Schäfer, K.H. Glassmeier, P.T.I. Eriksson, P.N. Mager, V. Pierrard, K.H. Fornaçon, L.G. Blomberg, Spatiotemporal structure of a poloidal Alfvén wave detected by Cluster adjacent to the dayside plasmapause. Ann. Geophys. 26, 1805-1817 (2008). doi:10.5194/angeo-26-1805-2008

A. Schekotov, V. Pilipenko, K. Shiokawa, E. Fedorov, ULF impulsive magnetic response at mid-latitudes to lightning activity. Earth Planets Space 63, 119-128 (2011). doi:10.5047/eps.2010.12.009

J.M. Schmidt, L. Ofman, Global simulation of an extreme ultraviolet imaging telescope wave. Astrophys. J. 713, 1008-1015 (2010). doi:10.1088/0004-637X/713/2/1008

C.J. Schrijver, D.S. Brown, Oscillations in the magnetic field of the solar corona in response to flares near the photosphere. Astrophys. J. Lett. 537, 69-72 (2000). doi:10.1086/312753

M. Selwa, L. Ofman, K. Murawski, Numerical simulations of slow standing waves in a curved solar coronal loop. Astrophys. J. Lett. 668, 83-86 (2007). doi:10.1086/522602

M. Selwa, S.K. Solanki, L. Ofman, The role of active region loop geometry. II. Symmetry breaking in threedimensional active region: why are vertical kink oscillations observed so rarely? Astrophys. J. 728, 87 (2011). doi:10.1088/0004-637X/728/2/87

R.P. Sharma, N. Yadav, N. Pathak, Role of 3d-dispersive Alfvén waves in coronal heating. Astrophys. Space Sci. 351, 75-80 (2014). doi:10.1007/s10509-014-1845-7

K. Shibasaki, High-beta disruption in the solar atmosphere. Astrophys. J. 557, 326-331 (2001). doi:10.1086/ 321651

K. Shibata, T. Magara, Solar flares: magnetohydrodynamic processes. Living Rev. Sol. Phys. 8, 6 (2011). doi:10.12942/lrsp-2011-6

D.G. Sibeck, G. Korotova, D.L. Turner, V. Angelopoulos, K.-H. Glaßmeier, J.P. McFadden, Frequency doubling and field-aligned ion streaming in a long-period poloidal pulsation. J. Geophys. Res. Space Phys. 117, 11215 (2012). doi:10.1029/2011JA017473

R. Soler, R. Oliver, J.L. Ballester, Magnetohydrodynamic waves in a partially ionized filament thread. Astrophys. J. 699, 1553-1562 (2009). doi:10.1088/0004-637X/699/2/1553 
R. Soler, R. Oliver, J.L. Ballester, Spatial damping of propagating kink waves in prominence threads. Astrophys. J. 726, 102 (2011). doi:10.1088/0004-637X/726/2/102

R. Soler, J. Andries, M. Goossens, Resonant Alfvén waves in partially ionized plasmas of the solar atmosphere. Astron. Astrophys. 537, 84 (2012). doi:10.1051/0004-6361/201118235

R. Soler, M. Goossens, J. Terradas, R. Oliver, The behavior of transverse waves in nonuniform solar flux tubes. I. Comparison of ideal and resistive results. Astrophys. J. 777, 158 (2013). doi:10.1088/ 0004-637X/777/2/158

D.J. Southwood, Some features of field line resonances in the magnetosphere. Planet. Space Sci. 22, 483-491 (1974). doi:10.1016/0032-0633(74)90078-6

D.J. Southwood, M.A. Saunders, Curvature coupling of slow and Alfvén MHD waves in a magnetotail field configuration. Planet. Space Sci. 33, 127-134 (1985). doi:10.1016/0032-0633(85)90149-7

K. Stasiewicz, P. Bellan, C. Chaston, C. Kletzing, R. Lysak, J. Maggs, O. Pokhotelov, C. Seyler, P. Shukla, L. Stenflo, A. Streltsov, J.-E. Wahlund, Small scale Alfvénic structure in the Aurora. Space Sci. Rev. 92 , 423-533 (2000)

R.S. Steinolfson, J.M. Davila, Coronal heating by the resonant absorption of Alfvén waves-importance of the global mode and scaling laws. Astrophys. J. 415, 354-363 (1993). doi:10.1086/173169

A.V. Stepanov, V.V. Zaitsev, Quasi-periodic pulsations and diagnostics of flaring plasma. Geomagn. Aeron. 54, 969-981 (2014). doi:10.1134/S0016793214080167

A.V. Stepanov, V.V. Zaitsev, V.M. Nakariakov, Coronal seismology. Phys. Usp. 55(27), 4 (2012). doi:10.3367/ UFNe.0182.201209f.0999

V.V. Surkov, M. Hayakawa, Ultra and Extremely Low Frequency Electromagnetic Fields (Springer, Tokyo, 2014). doi:10.1007/978-4-431-54367-1

R. Sych, V.M. Nakariakov, M. Karlicky, S. Anfinogentov, Relationship between wave processes in sunspots and quasi-periodic pulsations in active region flares. Astron. Astrophys. 505, 791-799 (2009). doi:10.1051/0004-6361/200912132

T. Tajima, J. Sakai, H. Nakajima, T. Kosugi, F. Brunel, M.R. Kundu, Current loop coalescence model of solar flares. Astrophys. J. 321, 1031-1048 (1987). doi:10.1086/165694

K. Takahashi, A.Y. Ukhorskiy, Solar wind control of Pc5 pulsation power at geosynchronous orbit. J. Geophys. Res. Space Phys. 112, 11205 (2007). doi:10.1029/2007JA012483

K. Takahashi, K.-H. Glassmeier, V. Angelopoulos, J. Bonnell, Y. Nishimura, H.J. Singer, C.T. Russell, Multisatellite observations of a giant pulsation event. J. Geophys. Res. Space Phys. 116, 11223 (2011). doi:10.1029/2011JA016955

Y. Taroyan, R. Erdélyi, J.G. Doyle, S.J. Bradshaw, Footpoint excitation of standing acoustic waves in coronal loops. Astron. Astrophys. 438, 713-720 (2005). doi:10.1051/0004-6361:20052794

M.G.G.T. Taylor, H. Hasegawa, B. Lavraud, T. Phan, C.P. Escoubet, M.W. Dunlop, Y.V. Bogdanova, A.L. Borg, M. Volwerk, J. Berchem, O.D. Constantinescu, J.P. Eastwood, A. Masson, H. Laakso, J. Soucek, A.N. Fazakerley, H.U. Frey, E.V. Panov, C. Shen, J.K. Shi, D.G. Sibeck, Z.Y. Pu, J. Wang, J.A. Wild, Spatial distribution of rolled up kelvin-Helmholtz vortices at earth's dayside and flank magnetopause. Ann. Geophys. 30(6), 1025-1035 (2012). doi:10.5194/angeo-30-1025-2012. http://www. ann-geophys.net/30/1025/2012/

J. Terradas, R. Molowny-Horas, E. Wiehr, H. Balthasar, R. Oliver, J.L. Ballester, Two-dimensional distribution of oscillations in a quiescent solar prominence. Astron. Astrophys. 393, 637-647 (2002). doi:10.1051/0004-6361:20020967

J. Terradas, R. Oliver, J.L. Ballester, On the excitation of trapped and leaky modes in coronal slabs. Astron. Astrophys. 441, 371-378 (2005). doi:10.1051/0004-6361:20053198

J. Terradas, J. Andries, M. Goossens, On the excitation of leaky modes in cylindrical loops. Sol. Phys. 246, 231-242 (2007). doi:10.1007/s11207-007-9067-6

J. Terradas, J. Andries, M. Goossens, I. Arregui, R. Oliver, J.L. Ballester, Nonlinear instability of kink oscillations due to shear motions. Astrophys. J. Lett. 687, 115-118 (2008a). doi:10.1086/593203

J. Terradas, I. Arregui, R. Oliver, J.L. Ballester, J. Andries, M. Goossens, Resonant absorption in complicated plasma configurations: applications to multistranded coronal loop oscillations. Astrophys. J. 679, 16111620 (2008b). doi:10.1086/586733

J. Terradas, M. Goossens, G. Verth, Selective spatial damping of propagating kink waves due to resonant absorption. Astron. Astrophys. 524, 23 (2010). doi:10.1051/0004-6361/201014845

B.J. Thompson, J.B. Gurman, W.M. Neupert, J.S. Newmark, J.-P. Delaboudinière, O.C. St. Cyr, S. Stezelberger, K.P. Dere, R.A. Howard, D.J. Michels, SOHO/EIT observations of the 1997 April 7 coronal transient: possible evidence of coronal Moreton waves. Astrophys. J. Lett. 517, 151-154 (1999). doi: $10.1086 / 312030$

D.J. Thomson, L.J. Lanzerotti, C.G. Maclennan, B. Heber, H. Kunow, R.E. Gold, Coherence of charged particle oscillations in the heliosphere $(\mathrm{f} \approx 5 \mu \mathrm{Hz})$ : implications for a solar modulation source. J. Geophys. Res. 106, 29341-29354 (2001). doi:10.1029/2001JA000011 
J. Threlfall, C.E. Parnell, I. De Moortel, K.G. McClements, T.D. Arber, Nonlinear wave propagation and reconnection at magnetic X-points in the Hall MHD regime. Astron. Astrophys. 544, 24 (2012). doi:10.1051/0004-6361/201219098

J. Threlfall, I. De Moortel, S.W. McIntosh, C. Bethge, First comparison of wave observations from CoMP and AIA/SDO. Astron. Astrophys. 556, 124 (2013). doi:10.1051/0004-6361/201321782

J.O. Thurgood, R.J. Morton, J.A. McLaughlin, First direct measurements of transverse waves in solar polar plumes using SDO/AIA. Astrophys. J. Lett. 790, 2 (2014). doi:10.1088/2041-8205/790/1/L2

V.T. Tikhonchuk, V.Y. Bychenkov, Effect of anomalous resistivity on MHD wave damping. J. Geophys. Res. 100, 9535-9538 (1995). doi:10.1029/95JA00520

S. Tomczyk, S.W. McIntosh, Time-distance seismology of the solar corona with CoMP. Astrophys. J. 697, 1384-1391 (2009). doi:10.1088/0004-637X/697/2/1384

S. Tomczyk, S.W. McIntosh, S.L. Keil, P.G. Judge, T. Schad, D.H. Seeley, J. Edmondson, Alfvén waves in the solar corona. Science 317, 1192 (2007). doi:10.1126/science.1143304

V.Y. Trakhtengertz, A.Y. Feldstein, About dissipation of Alfvén waves in the layer with anomalous resistance. Geomagn. Aeron. 25, 334-336 (1985)

V.A. Troitskaya, History of the study of upstream sources of ULF waves, in Geophysical Monograph, ed. by M.J. Engebretson, K. Takahashi, M. Scholer. Am, vol. 81 (Geophys, Union, Washington, 1994), p. 45

V.A. Troitskaya, A.V. Gul'elmi, Geomagnetic micropulsations and diagnostics of the magnetosphere. Space Sci. Rev. 7, 689-768 (1967). doi:10.1007/BF00542894

D. Tsiklauri, J.-I. Sakai, S. Saito, Particle-in-cell simulations of circularly polarised Alfvén wave phase mixing: a new mechanism for electron acceleration in collisionless plasmas. Astron. Astrophys. 435, 11051113 (2005). doi:10.1051/0004-6361:20042436

Y. Uchida, Propagation of hydromagnetic disturbances in the solar corona and Moreton's wave phenomenon. Sol. Phys. 4, 30-44 (1968). doi:10.1007/BF00146996

J. Vaclavik, K. Appert, Theory of plasma heating by low frequency waves: magnetic pumping and Alfvén resonance heating. Nucl. Fusion 31(10), 1945 (1991). http://stacks.iop.org/0029-5515/31/i=10/a=013

T. Van Doorsselaere, J. Andries, S. Poedts, M. Goossens, Damping of coronal loop oscillations. Calculation of resonantly damped kink oscillations of one-dimensional nonuniform loops. Astrophys. J. 606, 12231232 (2004a)

T. Van Doorsselaere, A. Debosscher, J. Andries, S. Poedts, The effect of curvature on quasi-modes in coronal loops. Astron. Astrophys. 424, 1065-1074 (2004b). doi:10.1051/0004-6361:20041239

T. Van Doorsselaere, V.M. Nakariakov, E. Verwichte, Detection of waves in the solar corona: kink or Alfvén? Astrophys. J. Lett. 676, 73-75 (2008c). doi:10.1086/587029

T. Van Doorsselaere, C.S. Brady, E. Verwichte, V.M. Nakariakov, Seismological demonstration of perpendicular density structuring in the solar corona. Astron. Astrophys. 491, 9-12 (2008b). doi:10.1051/ 0004-6361:200810659

T. Van Doorsselaere, V.M. Nakariakov, P.R. Young, E. Verwichte, Coronal magnetic field measurement using loop oscillations observed by Hinode/EIS. Astron. Astrophys. 487, 17-20 (2008c). doi:10.1051/ 0004-6361:200810186

T. Van Doorsselaere, N. Wardle, G. Del Zanna, K. Jansari, E. Verwichte, V.M. Nakariakov, The first measurement of the adiabatic index in the solar corona using time-dependent spectroscopy of Hinode/EIS observations. Astrophys. J. 727, 32 (2011). doi:10.1088/2041-8205/727/2/L32

S. Vasheghani Farahani, T. Van Doorsselaere, E. Verwichte, V.M. Nakariakov, Propagating transverse waves in soft X-ray coronal jets. Astron. Astrophys. 498, 29-32 (2009). doi:10.1051/0004-6361/200911840

S. Vasheghani Farahani, C. Hornsey, T. Van Doorsselaere, M. Goossens, Frequency and damping rate of fast sausage waves. Astrophys. J. 781, 92 (2014). doi:10.1088/0004-637X/781/2/92

A.M. Veronig, M. Temmer, B. Vršnak, High-cadence observations of a global coronal wave by STEREO EUVI. Astrophys. J. Lett. 681, 113-116 (2008). doi:10.1086/590493

A.M. Veronig, N. Muhr, I.W. Kienreich, M. Temmer, B. Vršnak, First observations of a dome-shaped large-scale coronal extreme-ultraviolet wave. Astrophys. J. Lett. 716, 57-62 (2010). doi:10.1088/ 2041-8205/716/1/L57

G. Verth, J. Terradas, M. Goossens, Observational evidence of resonantly damped propagating kink waves in the solar corona. Astrophys. J. Lett. 718, 102-105 (2010). doi:10.1088/2041-8205/718/2/L102

E. Verwichte, M.J. Aschwanden, T. Van Doorsselaere, C. Foullon, V.M. Nakariakov, Seismology of a large solar coronal loop from EUVI/STEREO observations of its transverse oscillation. Astrophys. J. 698, 397-404 (2009). doi:10.1088/0004-637X/698/1/397

E. Verwichte, T. Van Doorsselaere, R.S. White, P. Antolin, Statistical seismology of transverse waves in the solar corona. Astron. Astrophys. 552, 138 (2013). doi:10.1051/0004-6361/201220456

N.M. Viall, L. Kepko, H.E. Spence, Relative occurrence rates and connection of discrete frequency oscillations in the solar wind density and dayside magnetosphere. J. Geophys. Res. Space Phys. 114, 1201 (2009). doi:10.1029/2008JA013334 
J. Vogt, Alfvén wave coupling in the auroral current circuit. Surv. Geophys. 23, 335-377 (2002)

Y.M. Voitenko, Excitation of kinetic Alfvén waves in a flaring loop. Sol. Phys. 182, 411-430 (1998). doi:10.1023/A:1005049006572

Y. Voitenko, M. Goossens, Nonlinear decay of phase-mixed Alfvén waves in the solar corona. Astron. Astrophys. 357, 1073-1085 (2000)

A.D.M. Walker, The Kelvin-Helmholtz instability in the low-latitude boundary layer. Planet. Space Sci. 29, 1119-1133 (1981). doi:10.1016/0032-0633(81)90011-8

A.D.M. Walker, MHD Waves in Geospace (Institute of Physics Publishing, London, 2005)

Y.-M. Wang, EIT waves and fast-mode propagation in the solar corona. Astrophys. J. Lett. 543, 89-93 (2000). doi: $10.1086 / 318178$

T. Wang, Standing slow-mode waves in hot coronal loops: observations, modeling, and coronal seismology. Space Sci. Rev. 158, 397-419 (2011). doi:10.1007/s11214-010-9716-1

T.J. Wang, S.K. Solanki, Vertical oscillations of a coronal loop observed by TRACE. Astron. Astrophys. 421, 33-36 (2004). doi:10.1051/0004-6361:20040186

T. Wang, S.K. Solanki, W. Curdt, D.E. Innes, I.E. Dammasch, Doppler shift oscillations of hot solar coronal plasma seen by SUMER: a signature of loop oscillations? Astrophys. J. Lett. 574, 101-104 (2002). doi: $10.1086 / 342189$

T. Wang, D.E. Innes, J. Qiu, Determination of the coronal magnetic field from hot-loop oscillations observed by SUMER and SXT. Astrophys. J. 656, 598-609 (2007). doi:10.1086/510424

T. Wang, L. Ofman, J.M. Davila, Y. Su, Growing transverse oscillations of a multistranded loop observed by SDO/AIA. Astrophys. J. Lett. 751, 27 (2012). doi:10.1088/2041-8205/751/2/L27

A. Warmuth, G. Mann, Kinematical evidence for physically different classes of large-scale coronal EUV waves. Astron. Astrophys. 532, 151 (2011). doi:10.1051/0004-6361/201116685

D.F. Webb, T.A. Howard, Coronal mass ejections: observations. Living Rev. Sol. Phys. 9, 3 (2012). doi:10. 12942/lisp-2012-3

R.S. White, E. Verwichte, C. Foullon, First observation of a transverse vertical oscillation during the formation of a hot post-flare loop. Astron. Astrophys. 545, 129 (2012). doi:10.1051/0004-6361/201219856

R.S. White, E. Verwichte, C. Foullon, Anti-phase signature of flare generated transverse loop oscillations. Astrophys. J. 774, 104 (2013). doi:10.1088/0004-637X/774/2/104

D.R. Williams, M. Mathioudakis, P.T. Gallagher, K.J.H. Phillips, R.T.J. McAteer, F.P. Keenan, P. Rudawy, A.C. Katsiyannis, An observational study of a magneto-acoustic wave in the solar corona. Mon. Not. R. Astron. Soc. 336, 747-752 (2002). doi:10.1046/j.1365-8711.2002.05764.x

J. Woch, R. Lundin, Temporal magnetosheath plasma injection observed with Viking —a case study. Ann. Geophys. 9, 133-142 (1991)

J. Woch, G. Kremser, A. Korth, A comprehensive investigation of compressional ULF waves observed in the ring current. J. Geophys. Res. 95, 15113-15132 (1990). doi:10.1029/JA095iA09p15113

A.N. Wright, Dispersion and wave coupling in inhomogeneous MHD waveguides. J. Geophys. Res. 99, 159167 (1994). doi:10.1029/93JA02206

D.M. Wright, T.K. Yeoman, I.J. Rae, J. Storey, A.B. Stockton-Chalk, J.L. Roeder, K.J. Trattner, Groundbased and polar spacecraft observations of a giant (Pg) pulsation and its associated source mechanism. J. Geophys. Res. 106, 10837-10852 (2001). doi:10.1029/2001JA900022

J.R. Wygant, A. Keiling, C.A. Cattell, M. Johnson, R.L. Lysak, M. Temerin, F.S. Mozer, C.A. Kletzing, J.D. Scudder, W. Peterson, C.T. Russell, G. Parks, M. Brittnacher, G. Germany, J. Spann, Polar spacecraft based comparisons of intense electric fields and Poynting flux near and within the plasma sheet-tail lobe boundary to UVI images: an energy source for the aurora. J. Geophys. Res. 105, 18675 (2000). doi:10.1029/1999JA900500

T.K. Yeoman, D.M. Wright, L.J. Baddeley, Ionospheric signatures of ULF waves: active radar techniques, in Magnetospheric ULF Waves: Synthesis and New Directions, ed. by K. Takahashi, P.J. Chi, R.E. Denton, R.L. Lysak. Washington DC American Geophysical Union Geophysical Monograph Series, vol. 169 (2006), p. 273

T.K. Yeoman, M. James, P.N. Mager, D.Y. Klimushkin, SuperDARN observations of high-m ULF waves with curved phase fronts and their interpretation in terms of transverse resonator theory. J. Geophys. Res. Space Phys. 117, 6231 (2012). doi:10.1029/2012JA017668

S. Yu, V.M. Nakariakov, L.A. Selzer, B. Tan, Y. Yan, Quasi-periodic wiggles of microwave zebra structures in a solar flare. Astrophys. J. 777, 159 (2013). doi:10.1088/0004-637X/777/2/159

D. Yuan, V.M. Nakariakov, Measuring the apparent phase speed of propagating EUV disturbances. Astron. Astrophys. 543, 9 (2012). doi:10.1051/0004-6361/201218848

D. Yuan, V.M. Nakariakov, N. Chorley, C. Foullon, Leakage of long-period oscillations from the chromosphere to the corona. Astron. Astrophys. 533, 116 (2011). doi:10.1051/0004-6361/201116933

D. Yuan, Y. Shen, Y. Liu, V.M. Nakariakov, B. Tan, J. Huang, Distinct propagating fast wave trains associated with flaring energy releases. Astron. Astrophys. 554, 144 (2013). doi:10.1051/0004-6361/201321435 
K. Yumoto, V. Pilipenko, E. Fedorov, N. Kurneva, M. De Lauretis, K Kitamura, Magnetospheric ULF wave phenomena stimulated by SSC. J. Geomagn. Geoelectr. 49(10), 1179-1195 (1997)

V.V. Zaitsev, A.V. Stepanov, On the origin of the hard X-ray pulsations during solar flares. Sov. Astron. Lett. 8, 132-134 (1982)

V.V. Zaitsev, A.V. Stepanov, Coronal magnetic loops. Phys. Usp. 51, 1123-1160 (2008). doi:10.1070/ PU2008v051n11ABEH006657

I.V. Zimovets, V.M. Nakariakov, Excitation of kink oscillations of coronal loops: statistical study. Astron. Astrophys. 577, 4 (2015). doi:10.1051/0004-6361/201424960

I.V. Zimovets, A.B. Struminsky, Imaging observations of quasi-periodic pulsatory nonthermal emission in two-ribbon solar flares. Sol. Phys. 258, 69-88 (2009). doi:10.1007/s11207-009-9394-x

N.A. Zolotukhina, P.N. Mager, D.Y. Klimushkin, Pc5 waves generated by substorm injection: a case study. Ann. Geophys. 26, 2053-2059 (2008). doi:10.5194/angeo-26-2053-2008 